
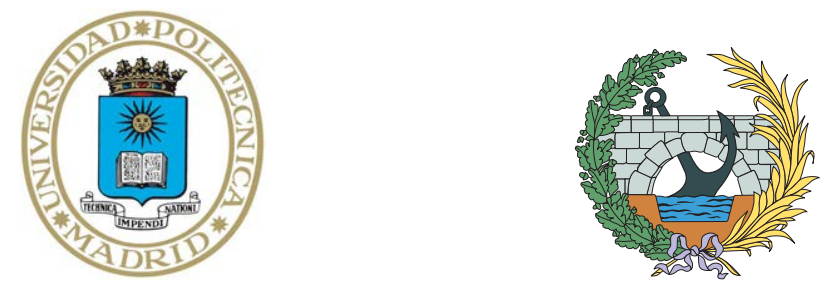

Universidad Politécnica de Madrid

Escuela Técnica Superior de

Ingenieros de Caminos, Canales y Puertos

\title{
Atomistic modeling of Ge damage accumulation, amorphization and solid phase epitaxial regrowth
}

Tesis doctoral

José Luis de Gómez-Sellés Ortuño

Licenciado en Ciencias Físicas

2015 


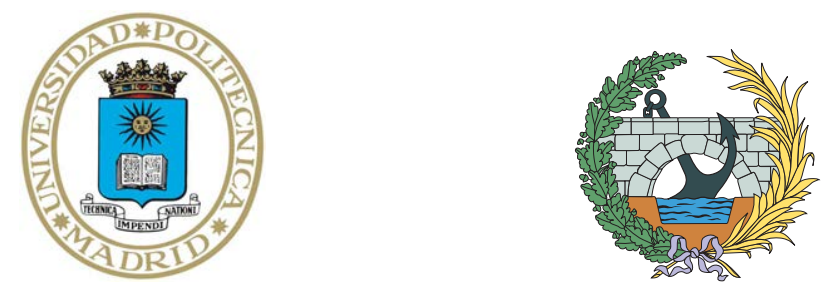

Departamento de Ciencia de Materiales

Escuela Técnica Superior de Ingenieros de Caminos, Canales y Puertos

Universidad Politécnica de Madrid

\title{
Atomistic modeling of Ge damage accumulation, amorphization and solid phase epitaxial regrowth
}

\author{
Tesis doctoral
}

José Luis de Gómez-Sellés Ortuño

Licenciado en Ciencias Físicas

Directores de la tesis

Ignacio Martín Bragado

Dr. en Ciencias Físicas

Javier Segurado Escudero

Dr. Ingeniero de Materiales

Profesor Titular de la Universidad 



\section{(3) \\ POLITÉCNICA}

Tribunal nombrado por el Sr. Rector Magfco. de la Universidad Politécnica de Madrid, el día... .de... ..de $20 \ldots$.

Presidente:

Vocal:

Vocal:

Vocal:

Secretario:

Suplente:

Suplente:

Realizado el acto de defensa y lectura de la Tesis el día..........de. .de $20 \ldots$ en la E.T.S.I. /Facultad

Calificación

EL PRESIDENTE

LOS VOCALES 

a Papá,

porque en silencio

te lo prometí. 

"Wir reiten in die Kreuz und Quer Nach Freuden und Geschäften Doch immer kläfft es hinterher Und bellt aus allen Kräften.

So will der Spitz aus unserm Stall Uns immerfort begleiten,

Und seines Bellens lauter Schall

Beweist nur, daß wir reiten".

— Johann W. von Goethe. Kläffer (1808). 



\section{Agradecimientos}

Quiero empezar agradeciendo al Dr. Ignacio Martín Bragado la confianza depositada en mí para empezar este proyecto, y al Instituto IMDEA Materiales por financiar y apoyar la finalización de esta Tesis. Me llevo todas las valiosas lecciones que he aprendido muy bien en estos más de tres años de trabajo para el resto de mi vida. Gracias al Dr. Javier Llorca y al Dr. Javier Segurado también. Gracias al Dr. Francis Benistant (GLOBALFOUNDRIES) por iniciar este proyecto.

El Dr. Blake Darby, Dr. Bradley Yates y Dr. Kevin Jones (Universidad de Florida) son altamente agradecidos por enseñarme, por primera vez, el valor de una excelente colaboración y confiar en mí para llevar a cabo una parte importante de su trabajo en las primeras fases de mi carrera investigadora. Fue, sin lugar a dudas, el período más desafiante y emocionante de este camino. Gracias al Dr. Pedro Castrillo (UCAM) por compartir conmigo sus amplios conocimientos y los buenos tiempos resolviendo el problema del nivel de Fermi. Fue realmente divertido. El Dr. Alain Claverie (CEMES/CNRS) es inmensamente agradecido por compartir desinteresadamente su sabiduría, recursos y tutela con lecciones extremadamente valiosas sobre esta cosa tan extraña llamada ciencia. Gracias también a la comunidad del CEMES: Bart, Marion, Iman, Thomas, Julien, David, Lucho, Luis Alfredo, Miguel, Victor, Mireille, Genevieve, Sandra, Richard y muchos otros por tomarme como una parte de su familia, y su apoyo incondicional. Gracias a aquellos que compartieron una parte de su preciado tiempo para ayudarme a entender todo lo que se me escapaba: Dr. Gerard Benassayag, Dr. Mathias Posselt, Dra. Simona Boninelli y Dra. Raquel González.

Me llevo conmigo el tiempo y las buenas experiencias compartidas con las personas que aunan el mayor talento del Instituto IMDEA Materiales: José Daniel Suarez, Nicolás Lara, 
Borja Casilda, Eva Aguado y Gema Sánchez. Gracias por todo vuestro apoyo. No quiero saber qué habría sido de mí sin vuestra constante ayuda y entendimiento en los tiempos difíciles. ¡Os deseo lo mejor! Miguel, José Luis, Vanesa, Marcos, Juan Pedro, Juan Pablo, Marta, Carmen, Paloma, Teresa, Edu, Vanessa, Mariana, Elena, Sergio, Gustavo, Alejandro, Luis, Irene, Mehdi, Eshan, Alicia, Marcos, Victor, Paqui, Alfonso, Pablo, Fernando, Arcadio, Jon... Todos vosotros. Hicisteis que estos años merecieran la pena.

La idea de convertirme en doctor nunca se me habría pasado por la cabeza sin la guía y grandes consejos de la Dra. Arántzazu Mascaraque, Dra. Gemma Piquero, y Dra. María Luisa Lucía Mulas. Gracias por eso y por todo lo demás.

Mil gracias tambiíen al mejor maestro que he tenido en mi vida, la persona que más física sabe del mundo: Fermo Fernández. Gracias por enseñarme que un 9,8 vale más que un 10. Gracias también por su labor en formarme como persona (o por lo menos intentarlo) a Miguel Ángel, Prado, Mari Carmen, Mike, Vicente, Manuel... Estoy muy orgulloso de haber pasado por vuestras manos.

A un nivel más personal, no puedo sino recordar, como hago cada día, las lecciones que aprendí de mi padre, Nicolás. Porque tenías razón. Porque no me puedo imaginar dónde estaría sin todo tu sacrificio. Porque you can't always get what you want, but if you try, sometimes, you just might find, you get what you need. ¡Ojalá estuvieras aquí! Gracias a mi madre, María José, por su amor y confianza en mí. Enseñándome que puedo alcanzar cualquier meta, y Luisa Abad por dármelo todo. Nicky, gracias por abrirme todo camino posible en la vida, y por tu constante protección. Gracias también a Luis Ortuño por estar siempre ahí.

El Sr. Javier Corrales es altamente agradecido por su eterna amistad, enseñándome how to die for that inch. Gracias a mis hermanos Jaime Azurmendi y Manuel Pozo.

Silvia, eres mi ejemplo. Tú marcas el camino, y yo te sigo. Gracias por empujar cuando ninguno de los dos teníamos fuerzas, y gracias por las sonrisas. Has caminado por el más espantoso de los caminos y has tenido lo necesario para conseguirlo. Gracias, gracias y un millón de veces gracias. 


\section{Resumen}

En los últimos años, el Ge ha ganado de nuevo atención con la finalidad de ser integrado en el seno de las existentes tecnologías de microelectrónica. Aunque no se le considera como un canddato capaz de reemplazar completamente al Si en el futuro próximo, probalemente servirá como un excelente complemento para aumentar las propiedades eléctricas en dispositivos futuros, especialmente debido a su alta movilidad de portadores. Esta integración requiere de un avance significativo del estado del arte en los procesos de fabricado. Técnicas de simulación, como los algoritmos de Monte Carlo cinético (KMC), proporcionan un ambiente atractivo para llevar a cabo investigación y desarrollo en este campo, especialmente en términos de costes en tiempo y financiación. En este estudio se han usado, por primera vez, técnicas de KMC con el fin entender el procesado "front-end" de Ge en su fabricación, específicamente la acumulación de dañado y amorfización producidas por implantación iónica y el crecimiento epitaxial en fase sólida (SPER) de las capas amorfizadas.

Primero, simulaciones de aproximación de clisiones binarias (BCA) son usadas para calcular el dañado causado por cada ión. La evolución de este dañado en el tiempo se simula usando KMC sin red, o de objetos (OKMC) en el que sólamente se consideran los defectos. El SPER se simula a través de una aproximación KMC de red (LKMC), siendo capaz de seguir la evolución de los átomos de la red que forman la intercara amorfo/cristalina. Con el modelo de amorfización desarrollado a lo largo de este trabajo, implementado en un simulador multi-material, se pueden simular todos estos procesos.

Ha sido posible entender la acumulación de dañado, desde la generación de defectos puntuales hasta la formación completa de capas amorfas. Esta acumulación ocurre en tres 
regímenes bien diferenciados, empezando con un ritmo lento de formación de regiones de dañado, seguido por una rápida relajación local de ciertas áreas en la fase amorfa donde ambas fases, amorfa y cristalina, coexisten, para terminar en la amorfización completa de capas extensas, donde satura el ritmo de acumulación. Dicha transición ocurre cuando la concentración de dañado supera cierto valor límite, el cual es independiente de las condiciones de implantación. Cuando se implantan los iones a temperaturas relativamente altas, el recocido dinámico cura el dañado previamente introducido y se establece una competición entre la generación de dañado y su disolución. Estos efectos se vuelven especialmente importantes para iones ligeros, como el $\mathrm{B}$, el cual crea dañado más diluido, pequeño y distribuido de manera diferente que el causado por la implantación de iones más pesados, como el Ge. Esta descripción reproduce satisfactoriamente la cantidad de dañado y la extensión de las capas amorfas causadas por implantación iónica reportadas en la bibliografía.

La velocidad de recristalización de la muestra previamente amorfizada depende fuertemente de la orientación del sustrato. El modelo LKMC presentado ha sido capaz de explicar estas diferencias entre orientaciones a través de un simple modelo, dominado por una única energía de activación y diferentes prefactores en las frecuencias de SPER dependiendo de las configuraciones de vecinos de los átomos que recristalizan. La formación de maclas aparece como una consecuencia de esta descripción, y es predominante en sustratos crecidos en la orientación (111)Ge. Este modelo es capaz de reproducir resultados experimentales para diferentes orientaciones, temperaturas y tiempos de evolución de la intercara amorfo/cristalina reportados por diferentes autores. Las parametrizaciones preliminares realizadas de los tensores de activación de tensiones son también capaces de proveer una buena correlación entre las simulaciones y los resultados experimentales de velocidad de SPER a diferentes temperaturas bajo una presión hidrostática aplicada.

Los estudios presentados en esta tesis han ayudado a alcanzar un mejor entendimiento de los mecanismos de producción de dañado, su evolución, amorfización y SPER para Ge, además de servir como una útil herramienta para continuar el trabajo en este campo. 


\section{Acknowledgments}

I want to first acknowledge Dr. Ignacio Martín Bragado the trust deposited on me to start this project, and IMDEA Materials Institute for funding and supporting the finalization of this dissertation. I will carry all the valuable lessons that I sure learned thorough these more than three years of work for the rest of my life. Thanks to Dr. Javier Llorca and Dr. Javier Segurado too. Thanks to Dr. Francis Benistant (GLOBALFOUNDRIES) for initiating this project.

Dr. Blake Darby, Dr. Bradley Yates and Dr. Kevin Jones (University of Florida) are well acknowledged for showing me, for the first time, the value of an excellent collaboration and relying on me to take care of an important part of their work at the first stages of my career as a researcher. It was, with no place for doubt, the most challenging and exciting period of this path. Thanks to Dr. Pedro Castrillo (UCAM) for sharing with me his broad knowledge, and the good times solving the Fermi level problem. It was real fun. Dr. Alain Claverie (CEMES/CNRS) is highly thanked for his selfless share of wisdom, resources and mentoring with extremely valuable lessons about this estrange thing called science. Thanks as well to the CEMES community: Bart, Marion, Iman, Thomas, Julien, David, Lucho, Luis Alfredo, Miguel, Victor, Mireille, Genevieve, Sandra, Richard and may others for taking myself as part of their family, and the unconditional support no matter what. Thanks to those who shared a piece of their precious time, to help me understand what escaped my mind: Dr. Gerard Benassayag, Dr. Mathias Posselt, Dr. Simona Boninelli and Dr. Raquel González.

I carry with me the time and good experiences shared with the best talented people at IMDEA Materials Institute: José Daniel Suarez, Nicolás Lara, Borja Casilda, Eva Aguado and Gema Sánchez. Thanks for all your support. I do not want to know what would have 
happened without your constant help and understanding in my darkest hours. I wish you all the best! Miguel, José Luis, Vanesa, Marcos, Juan Pedro, Juan Pablo, Marta, Carmen, Paloma, Teresa, Edu, Vanessa, Mariana, Elena, Sergio, Gustavo, Alejandro, Luis, Irene, Mehdi, Eshan, Alicia, Marcos, Victor, Paqui, Alfonso, Pablo, Fernando, Arcadio, Jon... All of you. You made that these years worthwhile.

The idea of becoming a $\mathrm{PhD}$ would have never crossed my mind without the guidance and good advise of Dr. Arántzazu Mascaraque, Dr. Gemma Piquero, and Dr. María Luisa Lucía Mulas. Thank you for that and all the rest.

Thanks also to the best teacher I have ever had, the person with the most broad knowledge in Physics: Fermo Fernández. Thank you for showing me that a 9,8 worths more than a 10. Thanks for their duties in forming me as a person (or at least tried) to Miguel Ángel, Prado, Mari Carmen, Mike, Vicente, Manuel... I am so proud of having passed through your hands.

At a more personal level, I cannot but remember, as I do every single day, the lessons that I learned from my father, Nicolás. Because you were right. Because I cannot imagine where would I be now without all your sacrifice. Because you can't always get what you want, but if you try, sometimes, you just might find, you get what you need. How I wish you were here! Thanks to my mother, María José, for her love and trust in me, teaching me that I can achieve any goal, and Luisa Abad for giving me everything. Nicky, thanks for opening me every possible path in life, and for your constant protection. Thanks also to Luis Ortuño for being always there.

Mr. Javier Corrales is highly thanked for his everlasting friendship, showing me how to die for that inch. Thanks to Jaime Azurmendi and Manuel Pozo, my brothers.

Silvia, you are my example. You lead the way, and I follow. Thanks for pushing when neither of us had the strength, and thanks for your smiles. You have been through the most terrifying road that one can ever ride, and still have what it takes to make it. Thanks, thanks, and a million times thanks. 


\section{Abstract}

In the recent years, Ge has regained attention to be integrated into existing microelectronic technologies. Even though it is not thought to be a feasible full replacement to Si in the near future, it will likely serve as an excellent complement to enhance electrical properties in future devices, specially due to its high carrier mobilities. This integration requires a significant upgrade of the state-of-the-art of regular manufacturing processes. Simulation techniques, such as kinetic Monte Carlo (KMC) algorithms, provide an appealing environment to research and innovation in the field, specially in terms of time and funding costs. In the present study, KMC techniques are used, for the first time, to understand Ge front-end processing, specifically damage accumulation and amorphization produced by ion implantation and Solid Phase Epitaxial Regrowth (SPER) of the amorphized layers.

First, Binary Collision Approximation (BCA) simulations are used to calculate the damage caused by every ion. The evolution of this damage over time is simulated using non-lattice, or Object, KMC (OKMC) in which only defects are considered. SPER is simulated through a Lattice KMC (LKMC) approach, being able to follow the evolution of the lattice atoms forming the amorphous/crystalline interface. With the amorphization model developed in this work, implemented into a multi-material process simulator, all these processes can be simulated.

It has been possible to understand damage accumulation, from point defect generation up to full amorphous layers formation. This accumulation occurs in three differentiated regimes, starting at a slow formation rate of the damage regions, followed by a fast local relaxation of areas into the amorphous phase where both crystalline and amorphous 
phases coexist, ending in full amorphization of extended layers, where the accumulation rate saturates. This transition occurs when the damage concentration overcomes a certain threshold value, which is independent of the implantation conditions. When implanting ions at relatively high temperatures, dynamic annealing takes place, healing the previously induced damage and establishing a competition between damage generation and its dissolution. These effects become specially important for light ions, as B, for which the created damage is more diluted, smaller and differently distributed than that caused by implanting heavier ions, as Ge. This description successfully reproduces damage quantity and extension of amorphous layers caused by means of ion implantation reported in the literature.

Recrystallization velocity of the previously amorphized sample strongly depends on the substrate orientation. The presented LKMC model has been able to explain these differences between orientations through a simple model, dominated by one only activation energy and different prefactors for the SPER rates depending on the neighboring configuration of the recrystallizing atoms. Twin defects formation appears as a consequence of this description, and are predominant for (111)Ge oriented grown substrates. This model is able to reproduce experimental results for different orientations, temperatures and times of evolution of the amorphous/crystalline interface reported by different authors. Preliminary parameterizations for the activation strain tensors are able to also provide a good match between simulations and reported experimental results for SPER velocities at different temperatures under the appliance of hydrostatic pressure.

The studies presented in this thesis have helped to achieve a greater understanding of damage generation, evolution, amorphization and SPER mechanisms in Ge, and also provide a useful tool to continue research in this field. 


\section{Notation}

\section{List of Symbols}

$\boldsymbol{\alpha}$ Amorphous.

$\boldsymbol{\alpha} / \boldsymbol{c}$ Amorphous/crystalline interface.

c Crystalline.

$\mathbf{E}_{\mathbf{a}}$ Activation energy of the Arrhenius expression for the event a.

$\mathbf{E}_{\mathbf{d c}}$ Critical energy density.

$\mathbf{E}_{\mathbf{t}}$ Threshold displacement energy per atom.

$\mathbf{K}_{\mathbf{a}}$ Prefactor of the Arrhenius expression for the event a.

$\mathbf{k}_{\mathrm{B}}$ Boltzmann's constant.

$\boldsymbol{\mu}_{\boldsymbol{e}}$ Mobility of electrons.

$\boldsymbol{\mu}_{\boldsymbol{h}}$ Mobility of holes.

$\nu_{\boldsymbol{a}}$ Arrhenius rate associated with the event a.

$\lambda$ Migration distance.

$\mathbf{r}_{\mathbf{c}}$ Capture radius.

$\rho_{G e}$ Atomic density of Ge.

$\boldsymbol{\rho}_{\boldsymbol{t}}$ Amorphization threshold.

$\boldsymbol{\sigma}$ Stress state.

$\mathbf{V}^{*}$ Activation strain tensor.

$\mathbf{V}_{\|}$Parallel component of the activation strain tensor.

$\mathbf{V}_{\perp}$ Normal component of the activation strain tensor.

$\Omega_{G e}$ Molar volume of Ge. 


\section{List of Abreviations}

BCA: Binary Collision Approximation.

CDED: Critical Damage Energy Density model.

DD: Dislocation Dynamics.

DFT: Density Functional Theory.

EOR: End Of Range Defect.

FEM: Finite Element Method.

HRTEM: High Resolution Transmission Electron Microscopy.

KMC: Kinetic Monte Carlo.

MC: Monte Carlo.

MD: Molecular Dynamics.

MM: More Moore.

MMonCa: Modular Monte Carlo Simulator.

MOSFET: Metal-oxide-semiconductor field-effect transistor.

MtM: More than Moore.

LKMC: Lattice Kinetic Monte Carlo.

$\mathrm{LN}_{2} \mathrm{~T}$ : Liquid Nitrogen Temperature.

OKMC: Object Kinetic Monte Carlo.

OOP: Object Oriented Programming.

PVTEM: Plain View Transmission Electron Microscopy.

RBS: Rutherford Backscattering Spectrometry.

RT: Room Temperature.

SPER: Solid Phase Epitaxial Regrowth.

SRIM: Stopping and Range of Ions in Matter.

TEM: Transmission Electron Microscopy.

TCAD: Technology Computer Aided Design.

USJ: Ultra Shallow Junction.

XTEM: Cross Section Transmission Electron Microscopy. 


\section{Nomenclature}

$\{311\}$ Pure self-Interstitial type, rod-like, extended defect in the $\{311\}$ family of planes.

(abc) Crystallographic plane with a, b and c components.

$\{a b c\}$ Crystallographic family of planes with a, b and c components.

$[a b c]$ Crystallographic direction with $\mathrm{a}, \mathrm{b}$ and c components.

$<a b c\rangle$ Crystallographic family of directions with a, b and c components.

$(a b c) G e$ Ge substrate grown in the [abc] direction.

$A$ Dopant of A species lying in a substitutional position.

$A_{i} \quad$ Dopant of A species lying in an interstitial position.

$A P$ Amorphous Pocket.

$A V$ Dopant of A species paired with a Vacancy.

$C$ : Cluster.

$c M N N \quad$ Crystalline first $\mathrm{M}$ nearest neighbors.

$C_{I, V}$ Self-Interstitial, or Vacancy concentration.

$D_{\text {self }}$ Self-diffusivity.

$D_{I, V} \quad$ Self-Interstitial, or Vacancy diffusivity.

$D C_{I, V}$ Self-Interstitial, or Vacancy transport capacity.

$D L \quad$ Pure self-Interstitial type, disk-shaped, extended defect.

FP Frenel pair.

FT Frank-Turnbull.

$I$ Self-Interstitial defect.

$I_{n} \quad$ Pure self-Interstitial type extended defect formed by n elements.

$I_{n} V_{m}$ Amorphous Pocket formed by n self-Interstitials and $\mathrm{m}$ Vacancies.

Int: Interface between two materials or phases.

$M E: \quad$ Mesh Element.

MP: Mobile Particle.

$V$ Vacancy defect.

$V_{n} \quad$ Pure Vacancy type extended defect formed by n elements. 
XII 


\section{Contents}

\begin{tabular}{|l|l|}
\hline Agradecimientos & I
\end{tabular}

$\begin{array}{lll}\text { Resumen } & \text { III }\end{array}$

\begin{tabular}{lll}
\hline Acknowledgments & V \\
\hline
\end{tabular}

\begin{tabular}{llll}
\hline Abstract & VII
\end{tabular}

Notation IX IX I

$\begin{array}{lll}1 & \text { Introduction } & 1\end{array}$

1.1 Motivation . . . . . . . . . . . . . . . . . . . . . . . . . . 2

1.2 Objective and statement of research . . . . . . . . . . . . . . . . . . . . . 3

1.3 Background information . . . . . . . . . . . . . . . . . . . . . 4

$1.3 .1 \mathrm{Si}$ and $\mathrm{Ge} \ldots \ldots \ldots \ldots$. . . . . . . . . . . . . . . . . 4

1.3 .2 Doping semiconductors . . . . . . . . . . . . . . . 6

1.3 .3 Defects in semiconductors . . . . . . . . . . . . . . . . 8

1.3 .4 Damage accumulation . . . . . . . . . . . . . . . . 15

1.3 .5 Amorphization . . . . . . . . . . . . . . . . . 16

1.3 .6 Annealing . . . . . . . . . . . . . . . . . . . . . . . . 17

1.3 .7 Solid Phase Epitaxial Regrowth . . . . . . . . . . . . . . . . . 18

1.3 .8 End of Range Defects . . . . . . . . . . . . . . . . . . . . . . . . . . 20

1.3 .9 Why Kinetic Monte Carlo? . . . . . . . . . . . . . . . . . . . . . 21

1.3 .10 Summary . . . . . . . . . . . . . . . . . . . . 23 
$1.4 \quad$ Organization of the Dissertation . . . . . . . . . . . . . . . . . . . . . . 24

\begin{tabular}{ll}
\hline Part I: Methods & 27
\end{tabular}

2 Object Kinetic Monte Carlo 29

$2.1 \quad$ Introduction . . . . . . . . . . . . . . . . . . . . . . . . . . . . . . . . . . . . . . 29

2.2 Kinetic Monte Carlo . . . . . . . . . . . . . . . . . . . . . . . . . . . . . . 31

2.3 MMonCa $\ldots \ldots \ldots \ldots \ldots$

$2.3 .1 \quad$ Rates and time handling . . . . . . . . . . . . . . . . . . . . 34

$2.3 .2 \quad$ Space handling . . . . . . . . . . . . . . . . . . . 35

2.3 .3 OKMC: Defects . . . . . . . . . . . . . . . . 36

2.4 Some Examples . . . . . . . . . . . . . . . . . . . . . . . . . . . . . . . . . 42

2.4 .1 Theoretical particle diffusion . . . . . . . . . . . . . . . . . 42

$2.4 .2 \quad$ Si Extended Defects dissolution . . . . . . . . . . . . . . . . . . . . 44

$2.4 .3 \quad$ OKMC modeling of Si amorphization . . . . . . . . . . . . . . . . . 47

2.5 Conclusions . . . . . . . . . . . . . . . . . . . . . . . . . . . 47

3 Lattice Kinetic Monte Carlo 49

$3.1 \quad$ Introduction . . . . . . . . . . . . . . . . . . . . . . . . . . . . . . . . . 49

3.2 Modeling Solid Phase Epitaxial Regrowth . . . . . . . . . . . . . . . . 50

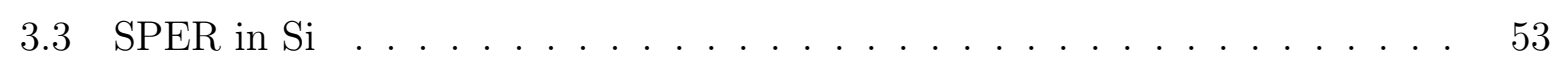

3.3 .1 Different orientations . . . . . . . . . . . . . . . . . . . . 53

3.3 .2 Twin defects . . . . . . . . . . . . . . . . . 55

3.3 .3 Hydrostatic pressure . . . . . . . . . . . . . . . . 57

3.4 Conclusions . . . . . . . . . . . . . . . . . . . . . . . . . . . . . . . . . . 58

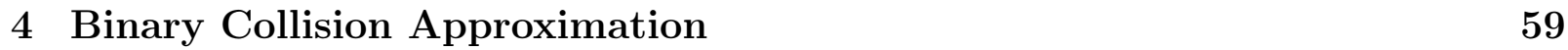

$4.1 \quad$ Introduction . . . . . . . . . . . . . . . . . . . . . . . . . . . . . . . . . . . . . . . 59

4.2 How BCA works? . . . . . . . . . . . . . . . . . . . . . . . . . . . . 60

$4.2 .1 \quad$ Nuclear collisions . . . . . . . . . . . . . . . . . . . . . . . 61

$4.2 .2 \quad$ Electronic Stopping . . . . . . . . . . . . . . . . . . . . 63

4.3 Conclusions $\ldots \ldots \ldots \ldots$ 
\begin{tabular}{ll}
\hline Part II: Results & 65
\end{tabular}

5 Amorphization in Ge by means of ion implantation 67

5.1 Introduction . . . . . . . . . . . . . . . . . . . . . . . . . . . 68

5.2 Model . . . . . . . . . . . . . . . . . . . . . . . . . . . . . . . . 69

5.3 Results and Discussion . . . . . . . . . . . . . . . . . . . . . . . . . . . 74

5.3 .1 Amorphization threshold . . . . . . . . . . . . . . . . 75

5.3 .2 Dynamic annealing . . . . . . . . . . . . . . . 80

5.3 .3 Differences between ions . . . . . . . . . . . . . . . . . . 80

5.3 .4 Cluster dissolution . . . . . . . . . . . . . . . . . 87

5.4 Limitations . . . . . . . . . . . . . . . . . . . . . . . . . . . . . . . 91

5.5 Conclusions $\ldots \ldots \ldots \ldots$. . . . . . . . . . . . . . . . . . . . . 92

6 Solid Phase Epitaxial Regrowth in Ge $\quad 95$

6.1 Introduction . . . . . . . . . . . . . . . . . . . . . . . 96

6.2 LKMC modeling . . . . . . . . . . . . . . . . . . . . . . . . . . . . 97

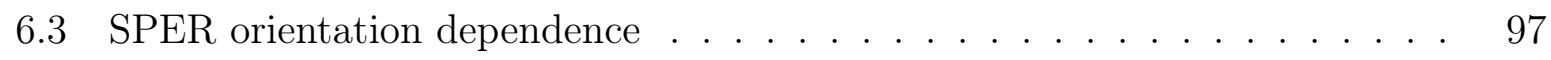

6.3 .1 Simulations . . . . . . . . . . . . . . . . . . . . . . . . 98

6.3 .2 Discussions . . . . . . . . . . . . . . . . . . . . 101

6.4 Other considerations . . . . . . . . . . . . . . . . . . . . 103

6.4 .1 Temperature calibration . . . . . . . . . . . . . . 103

6.4 .2 Planarization . . . . . . . . . . . . . . . . . 105

6.4.3 Stress-induced effects in Ge SPER: Hydrostatic Pressure . . . . . . 108

6.5 Limitations . . . . . . . . . . . . . . . . . . . . . . . . . . . . . . . . . . 110

6.6 Conclusions . . . . . . . . . . . . . . . . . . . . . . . . . . . . . . 110

$\begin{array}{lll}7 & \text { Concluding Comments } & 113\end{array}$

7.1 Damage accumulation and amorphization in Ge by means of ion implantation 113

7.2 Solid Phase Epitaxial Regrowth of Ge . . . . . . . . . . . . . . . . . . . . . 114

$\begin{array}{llr}8 & \text { Further Work } & 117\end{array}$

8.1 Amorphization . . . . . . . . . . . . . . . . . . . . . . . 117

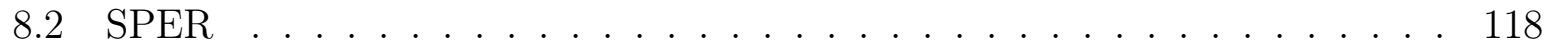


8.3 End of Range defects in $\mathrm{Ge} \quad \ldots \ldots \ldots \ldots$

\begin{tabular}{lr}
\hline Bibliography & 121
\end{tabular}

\begin{tabular}{ll}
\hline List of Figures & 135
\end{tabular}

\begin{tabular}{ll}
\hline List of Tables & 143
\end{tabular}

\begin{tabular}{|lr}
\hline A Running MMonCa & 145
\end{tabular}

A.1 Script Example . . . . . . . . . . . . . . . . . . . . . . . . 145

A.2 Parameters . . . . . . . . . . . . . . . . . . . . . . . . . . . 152

\begin{tabular}{lll}
\hline B Personal contributions & 159
\end{tabular} 


\section{Снаттв 1}

\section{INTRODUCTION}

"All great deeds and all great thoughts have a ridiculous beginning. Great works are often born on a street corner or in a restaurant's revolving door."

- Albert Camus

Semiconductor industry eagers to follow Moore's law, doubling the number of transistors in a dense integrated circuit approximately every two years, Moore et al. (1998). In order to achieve such goal, and mostly in the recent years, technologies have advanced through different approaches such as reducing the size of the components inside every chip (downscaling), changing geometry of transistor architectures or inducing stress in the materials to achieve better electrical properties.

Despite Si-based technologies have been dominant over the last decades, industrial needs require to consider different materials which, even not being thought to replace $\mathrm{Si}$ in the near future, their integration may increase performance of existing transistors (More than Moore, MtM) or even lead to a more aggressive downscaling of existing technologies (More Moore, MM). 


\subsection{Motivation}

The International Technology Roadmap for Semiconductors (ITRS) establish every year the needs and reported issues to be solved in order to achieve the desired Moore, MM, and MtM requirements. In the latest edition, published in 2013, ITRS (2013) it is remarked in the Front End Process Summary the need of solving issues related to:

- Continued scaling of non-planar High Performance multigate device in all aspects: Equivalent Oxide Thickness (EOT), junctions, mobility enhancement, new channel materials, parasitic series resistance, contact silicidation.

- Introduction and heterointegration of high mobility channels (based on III-V and Ge) to replace strained Si for continual performance.

- Continual EOT scaling and gate dielectrics with low $D_{I} T$, bulk traps and leakage for high mobility, low bandgap channels materials (Ge, III-V and 2D materials).

In all highlighted aspects, Ge can play an important role. As Ge may serve as a good candidate to be included in the existing technologies, further research should be conducted to solve the mentioned issues and pushing forward the existing capabilities. Even though Si models have been developed over the years, more fundamental research is still needed for Ge. Furthermore, existing models for SiGe alloys are normally extracted from interpolation between $\mathrm{Si}$ and Ge materials. Thus, a deeper research in Ge processing techniques will not only help to understand and improve existing Ge-based processes and devices, but will also give a direct impact in existing SiGe models.

In both the ITRS 2012th Edition update and 2013th Edition it is also remarked the importance of reliable Modeling and Simulation tools, usually referred as extended Technology Computer Aided Design (TCAD) as one of the few enabling methodologies that can reduce development cycle times and costs, covering the main topical areas leading to support technology development and optimization. Some of the reported needs and difficult challenges in the Modeling and Simulation Chapter span: 
- Coupled diffusion/(de)activation/damage/stress models and parameters including low-temperature, Solid Phase Epitaxial Regrowth (SPER), milisecond and microwave processes in Si-based substrate, that is, Si, SiGe, Ge-on-Si, III/V-on-Si [...].

- Implantation models (parameters) for ions needed for new materials

- Modeling of epitaxially grown layers: Morphology, stress, defects, doping, diffusion, activation.

- Modeling the impact of front-end processing-induced damage to devices on their leakage, noise and reliability behavior during operation.

- Physical models for novel channel materials, e. g., p-type Ge and compound III/V, especially concerning thin layers interfaces, defects and traps...

- Accurate, robust and computational efficient modeling of wide bandgap devices.

- Treatment of individual dopant atoms and traps in (commercial) continuum and Monte Carlo $(M C)$ device simulation. Coupling between atomistic process and continuum or atomistic device simulation.

Proposed solutions comprise cooperations between universities, independent research laboratories and companies, to provide models implemented in physically based, easy-toaccess and predictive tools.

For the expressed reasons, there is a justified need to model and understand the mechanisms that occur in semiconductor processing stages for both traditional and new materials to meet the industrial requirements.

\subsection{Objective and statement of research}

The goal of this work is to develop models for a physically based kinetic Monte Carlo (KMC) simulator, which are able to reproduce realistic scale material processing stages for the semiconductor industry. Such models should be able to shed light on Ge processing for microelectronics, including damage accumulation and amorphization by means of ion 
implantation and Solid Phase Epitaxial Regrowth (SPER) processes in Ge. The simulator shall also be calibrated to reproduce well known processes in Si dopant diffusion, defect formation and evolution, and SPER. More specifically, the following aspects have been studied:

- Active development of an Open Source, Object Oriented Programmed C++, physicallybased KMC process simulator. ${ }^{1}$

- Benchmarking and calibration of the models through reproduction of theoretical and well-known experimental results on Si extended defect evolution.

- Damage accumulation and dissolution mechanisms in Ge by means of ion implantation.

- Ge amorphization caused by different ions, extension of fully amorphize layers, doseindependent amorphization threshold.

- Atomistic calibration of SPER in Ge: growth rates substrate orientation dependence. Scalability of SPER stress dependence models from Si to Ge.

- Coupling Object-KMC (OKMC) and Lattice-KMC (LKMC) modules to simulate realistic full amorphization-plus-SPER processes.

To achieve such goals, a direct implication in the development and optimization of physically based models into a KMC simulator, MMonCa, has been done, implementing and calibrating the new models presented in this work, but also using existing previous models.

\subsection{Background information}

\subsubsection{Si and Ge}

Despite the first transistor was made from Ge due to its outstanding carrier mobility, issues such as instability of Ge's native oxide $\left(\mathrm{GeO}_{x}\right)$ or leakage in the metal contacts due

\footnotetext{
${ }^{1} \mathrm{~A}$ version of the simulator, MMonCa, can be downloaded at http://materials.imdea.org/MMonCa
} 
Table 1.1: Band Gap, and carrier mobilities of Si and Ge, Sze and Ng 2006)

\begin{tabular}{cccc}
\hline \hline & $\begin{array}{c}\text { Band Gap } \\
(\mathrm{eV})\end{array}$ & $\begin{array}{c}\mu_{e} \\
\left(\mathrm{~cm}^{2} / V s\right)\end{array}$ & $\begin{array}{c}\mu_{h} \\
\left(\mathrm{~cm}^{2} / V s\right)\end{array}$ \\
\cline { 2 - 4 } $\mathrm{Si}$ & 1.12 & 1500 & 600 \\
$\mathrm{Ge}$ & 0.66 & 3900 & 1900 \\
\hline \hline
\end{tabular}

to its low band gap, the semiconductor industry changed into Si technologies. Over the last decades, wide research has been performed in Si and materials which are integrated in Si-based technologies, but if industry requires to include new materials, further research is needed in order to understand and optimize these new components.

Ge-based devices may entail an increase in performance due to the lower temperatures needed in process stages when compared to $\mathrm{Si}$, in addition to the low band gap and high mobility, which makes Ge a very good candidate as channel main component material (see Table 1.1. Further studies on Ge, to understand and solve the issues that made the industry leave Ge-based technologies, will help specially into MtM approaches. For instance, recent implementation of high-k gate dielectric has circumvented the issues regarding the unstable native oxide of Ge.

Still, numerous uncertainties remain unexplained, such us formation and dissolution of defects in Ge at processing stages, Koffel et al. (2009); Boninelli et al. (2012a); Cowern et al. (2013) which are of special importance for performance and power leakage, and the mechanisms of dopant transport, coupled with their effects on real devices. As an example, $\mathrm{B}$ diffusion mechanisms in Ge have been under debate for the recent years until it was demonstrated that it diffuses through an Interstitial mediated mechanism, Mirabella et al. (2013) and refs. therein. This has opened recently possibilities of understanding issues that were before hidden, such as the real nature and behavior of Interstitials and Interstitialtype defects in Ge. Moreover, B implantation into Ge offers the possibility of very stable Ultra Shallow Junctions (USJ), due to its low diffusion in bulk Ge, Yates et al. (2012). 


\subsubsection{Doping semiconductors}

In order to achieve the desired electrical performance in microelectronic devices, atoms with different properties than the bulk material (or substrate) are introduced in some parts of the device, at the process semiconductor manufacturing step. This process is known as doping, and it can be performed in different ways. There is a extreme importance in controlling the dopant concentration, position (profiles) and behavior under all manufacturing process, to maintain performance and integrity of final products. Due to the scales used in the microelectronic industry, this need increases even more, for example, when compared to the higher scales considered in solar cells, where doping is performed by in-diffusion. This technique does not require high economic expenses, but versatility is not enough to our purposes. Thus, ion implantation is normally used in the microelectronic industry. This technique undergoes very complex mechanisms that are needed to be understood.

\section{Ion implantation}

Ion implantation consists in the bombardment of ionized atoms into a material with enough energy to penetrate beyond the most superficial layers, Katz and Sze (1983), but low enough to not pass through the material, where the term irradiation is then used. The objective of such implantation is, basically, the doping of the material. The idea of doping in semiconductor materials by ionic bombarding was first patented by William Shockley in 1954 in Bell laboratories. Later, in the 60s, ionic implantation technique was developed, being the mainstream way to introduce impurities in semiconductors, widely used and studied during the last decades.

The depth achieved by ions is approximately proportional to the initial energy, providing a parameter to control the doping profiles. This technique allows the doping of semiconductor materials with nearly every desired element, independent of its solubility into the target material. In other doping techniques, as in-diffusion, velocity and dose depend strongly on the solubility of the target element; while doping during epitaxial growth, gives rise to a constant impurity profile.

Ion implantation is a fast, homogeneous and reproducible process in which the number of implanted atoms and their energy is controlled with a high level of exactitude, which 


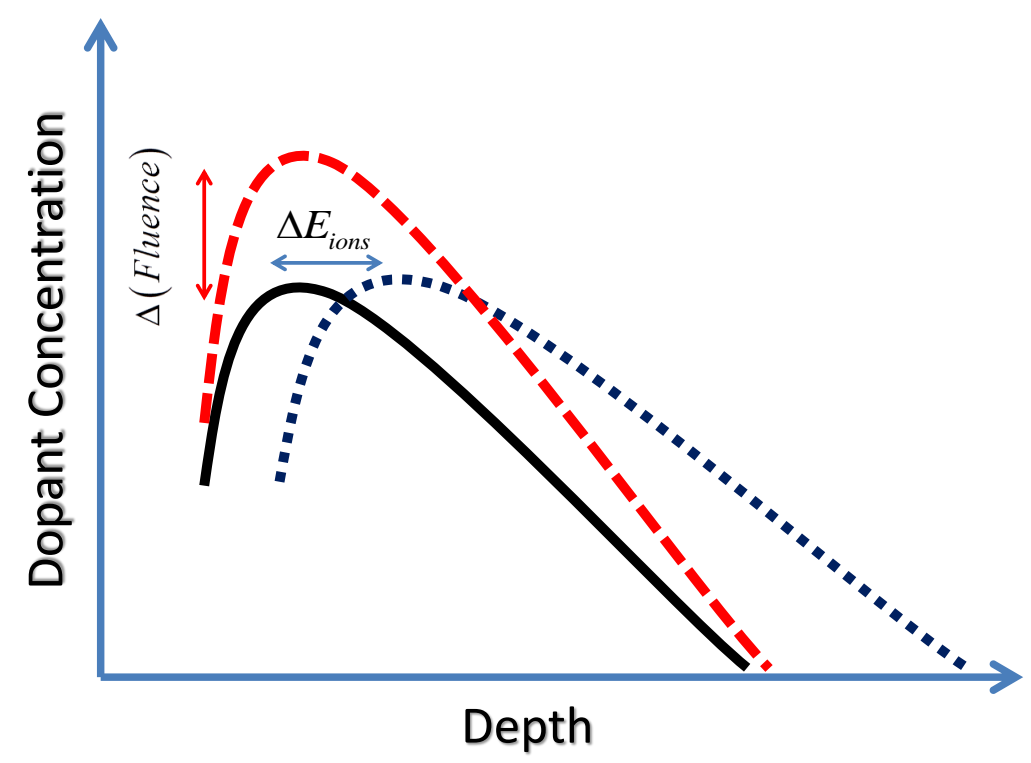

Figure 1.1: Qualitative representation of the impact caused by varying the energy of the implanted ions (blue dashed line) or the ion fluence (red dashed line), when compared to a reference profile (black solid line).

becomes important especially when low concentration of dopants is desired. In general, it allows to precisely control the implantation profile. It also presents a small lateral dispersion, which makes it possible to manufacture very small (low dimensional) devices maintaining a very low parasitic capacitance between them. In standard fabrication processes, it also allows to keep low temperatures, minimizing dopant diffusion.

Figure 1.1 is a qualitative representation of the different obtained profiles achieved by changing the implantation parameters. Starting from a dopant profile as shown in the black line, an increase in the energy of the ions will cause an in-depth shift of the profile (blue dashed line). If the fluence is increased, the overall dopant concentration will be higher, achieving a higher dopant profile (red dashed line). Units of dopant concentration are normally expressed in $\mathrm{cm}^{-3}$, while $\mathrm{x}$-axis is normally found in $\mathrm{nm}$ or $\mu \mathrm{m}$, depending on the scales.

Nevertheless, ion implantation has also some main disadvantages. The generated dam- 
age when implanting ions represents a problem, which increase for high doses. Defects appear in the lattice, changing the electrical properties of the substrate, and in the end, affecting the performance of the device.

The implanted dopants mostly occupy interstitial positions, which means that they will not be electrically active, forcing annealing at high temperatures to reorder the atoms into crystalline positions. This needed annealing makes the impurities to diffuse, changing the desired profile. Moreover, anomalous diffusion of some implanted species, as B, makes it even more complex to obtain abrupt profiles.

Another important effect is channeling of implanted ions in certain directions of the crystalline lattice. This characteristic enhances the complexity in terms of dopant profiles control and reproducibility.

\subsubsection{Defects in semiconductors}

As it has already been introduced, defects in semiconductors can play a very important role in device performance at all scales: from process manufacturing, to final device functionalities. Thus, it becomes important to understand the behavior of such entities at all relevant conditions. In this section, a brief introduction on the type of defects and their nature will be given focused on the important scales that this dissertation is written.

\section{Point Defects}

Point defects are the fundamental constituents of nearly every other more extended defect, and they play a role in their formation, evolution and dissolution. According to Fahey et al. (1989) Point defects can be divided into two sub-groups. Native point defects, and Dopant defects. 


\section{Native point defects}

The fundamental point defects that are considered, are Self-Interstitials ${ }^{2}(I)$, and Vacancies $(V)$. An $I$ is an atom of the lattice that has abandoned its natural lattice position, which is now occupied with a hole, that is commonly referred as a $V$. Extended point defects in both Si and Ge materials have been postulated, and also appear as an answer of different mechanisms reported in the literature. Nevertheless, their nature and behavior are still under discussion by different groups, Cowern et al. (2013); Kube et al. (2013).

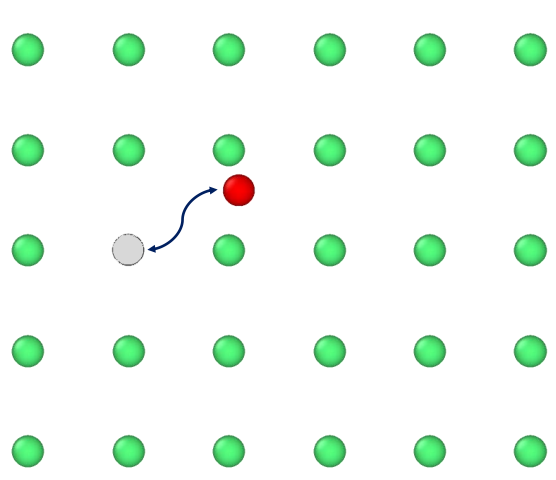

(a)

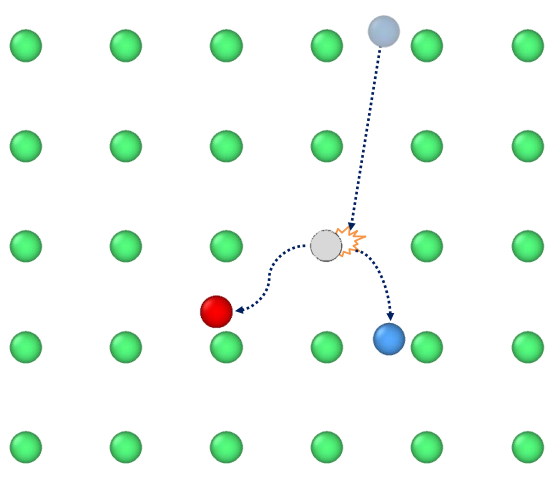

(b)

Figure 1.2: Sketches for $I$ (red) and $V$ (gray) formation. (a) Frenkel-pair formation within the bulk by thermal vibrations. (b) Atom displacement due to the impact of an incoming ion (blue). Lattice atoms are green-colored. Arrows indicate possible trajectories of the atoms.

Formation of such defects may be caused by three main mechanisms:

- Frenkel pair formation within the bulk. By thermal vibration, an atom can eventually leave its perfect lattice site position, to an $I$ position, leaving a $V$ behind, following the relation:

\footnotetext{
${ }^{2}$ In the following, self-interstitials will be named as interstitials, for notation issues. When other kind of interstitial defect is mentioned, the nature of that defect will be specified.
} 


$$
I+V \rightleftharpoons \varnothing
$$

as shown in Fig. 1.2(a), These two new defect as regularly referred as Frenkel pairs or FP. This process may be neglected for normal process conditions, since the activation barrier for creating both an $I$ and a $V$ is high at equilibrium.

- Atom displacement due to the impact of an incoming ion, or secondary recoil atoms. When the semiconductor material is being implanted, the incoming atoms will eventually hit lattice atoms. If the kinetic energy of the ion is high enough, the lattice atom will be displaced, thus creating a FP. A sketch of this process is shown in Fig. 1.2(b).

- Emission of $I$ s and $V \mathbf{s}$ by the material surface. Is can be created as well by an atom of the surface moving deeper within the bulk. In the same way, a lattice atom can also move to the surface, leaving a $V$ site at its former position. As the inverse process also occurs, there is an equilibrium concentration of native point defects in the material, that depends on the temperature.

Even though it is highly accepted that surface recombination occurs for both $I$ and $V$ native point defects in $\mathrm{Si}$, $\operatorname{Lim}(1995)$ in Ge, there is some controversy on whether Is recombine at the surface or not. While studies performed on B diffusion under proton irradiation in Ge suggest that the Ge surface might not be an effective sink for $I \mathrm{~s}$, Bracht et al. (2009) very recent studies on end-of-range defect dissolution in Ge after Solid Phase Epitaxial Regrowth point towards an effective surface recombination of $I$ s, Boninelli et al. (2012a).

Depending on the substrate temperature, these defects may migrate within the material. Moreover, this will be of extreme importance for transport of dopants.

Microscopically, diffusion of $I$ s can be understood as movements or migration steps within the free space existing between the lattice atoms, and $V$ s diffuse through an exchange mechanism between a lattice atom and the adjacent $V$. Macroscopically, diffusivity of native point defects is usually expressed in terms of self-diffusivity, taking into account 


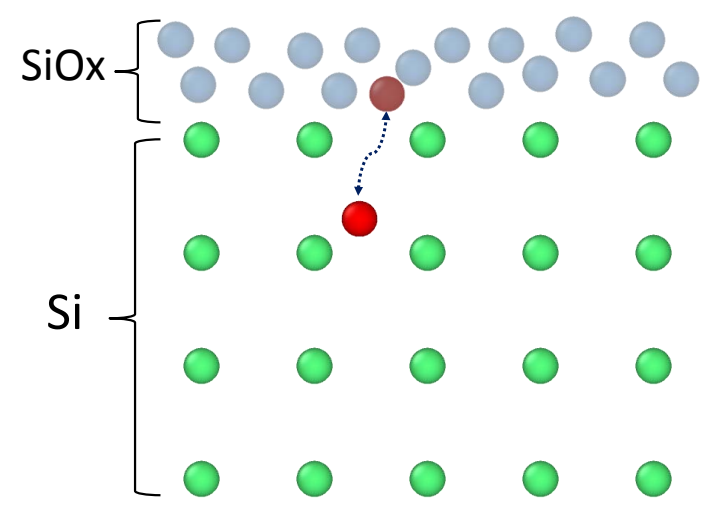

(a)

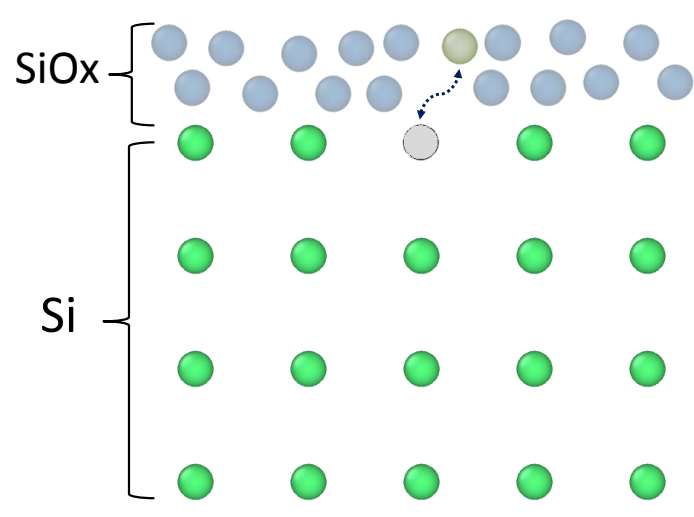

(b)

Figure 1.3: Sketches for $I$ (red) and $V$ (gray) emission and recombination in the surface. As a example, the interface between bulk lattice Si (green) atoms and an amorphous Silicon Oxide ( $\mathrm{SiOx}$ ) layer (blue) atoms is schematically represented for (a) Interstitial atoms emission and/or recombination in the $\mathrm{Si} / \mathrm{SiOx}$ interface, and (b) Vacancy sites emission and/or recombination in the $\mathrm{Si} / \mathrm{SiOx}$ interface. Arrows indicate possible trajectories of the atoms.

both the concentration $\left(C_{V}\right.$ and $\left.C_{I}\right)$ and diffusivity $\left(D_{I}\right.$ and $\left.D_{V}\right)$ of $I$ s and $V$ s respectively, through the following expression:

$$
D_{\text {self }}=f_{V} D C_{V}+f_{I} D C_{I}
$$

where $D C_{I}$ and $D C_{V}$ are the so called transport capacities, or $D C$-product, resulting from the product $D_{I} C_{I}$ or $D_{V} C_{V}$ for both $I$ s and $V$ s respectively. $f_{I}$ and $f_{V}$ are the correlation factors of $I$ s and $V$ s respectively, with values $f_{I} \simeq 0.7$ and $f_{V}=0.5$, following Bracht et al. (1998) and Posselt et al. (2008).

This notation of DC as the transport capacity is a result of the important role that these point defects play, assisting dopant diffusion. Moreover, in $\mathrm{Si}$, self-diffusion is mostly $I$-driven, Bracht et al. (1998) while in Ge, Vs are dominant, Bracht (2014). This will have an effective impact in dopant diffusion. 


\section{Dopant defects}

Defects formed by other elements than the substrate material are referred as dopant defects. They may occupy substitutional lattice sites (electrically active), or be in electrically inactive positions, such as interstitial or clustered forms. Understanding the mechanisms underlying the behavior of dopants is of high importance, as it allows to perform the socalled Point Defect Engineering, by controlling dopant quantity, position and electrical activation within the wafer. During this thesis, the following notation will be used: a dopant of a chemical species A in substitutional position will be referred as $A$, if it is in an interstitial position, $A_{i}$. A dopant can also form a bond with an $I$ if they are close enough, forming an $A I$-pair, and the same stands for the $A V$-pair. $A$ will be assumed as immobile, and the last three as mobile. Formation reactions of these pairs are:

$$
\begin{aligned}
& A+I \rightleftharpoons A_{i} \\
& A+I \rightleftharpoons A I \\
& A+V \rightleftharpoons A V \\
& A \rightleftharpoons A_{i}+V
\end{aligned}
$$

Reactions 1.3 and 1.4 are known as the kick-out mechanisms and are assumed to be similar, since distinction between them will not play a role. Thus, in this thesis, and for the sake of notation simpleness, $A_{i}$ will be used for referring to them. Reaction 1.5 is the $V$ mechanism, and 1.6 is the Frank-Turnbull mechanism of diffusion, which is energetically unfavorable, and thus will not play a role for dopant diffusion at the studied conditions.

As it will be shown in Chapter 2, diffusion of dopants will normally follow gaussian profiles. For extreme conditions of ion implantation in which damage and dopant concentrations are shallow and high, further mechanisms are needed to explain dopant profiles of non-gaussian shapes after thermal treatments, Mirabella et al. (2003). At these conditions, precipitates appear in the form of clusters such as $A_{n} I_{m}$ or $A_{n} V_{m}$.

Remembering that self-diffusion in $\mathrm{Si}$ is $I$-dominant, and $V$-dominant in Ge, it is now understandable that for example, $\mathrm{B}$, which diffuse interstitially assisted, will diffuse "easier" in Si than in Ge, Mirabella et al. (2013). 


\section{Clusters and Extended Defects}

Reactions between defects within the sample may produce bigger defects by agglomeration. These type of defects are commonly referred as clusters or extended defects. The nature of these defects, of course, depends on the constituent atoms, and their possible formation, evolution and dissolution may play an important role in very different processes, such as damage accumulation, amorphization or dopant diffusion. These mechanisms may be as simple as a single reaction (e.g. $I+I \rightarrow I_{2}$ ), or of extreme complexity, as Amorphous Pocket $(A P)$ formation by agglomeration of several $I \mathrm{~s}$ and $V \mathrm{~s}$, or Ostwald ripening, Ostwald (1900) of pure $I$ - or $V$-type clusters, vast void formation through coalescence of high amounts of $V$ s or $V$-type clusters, for example.

The behavior of these defects is often modeled through emission, or dissolution barriers which depend on the formation or binding energies of the constituents, and due to the fact that some of these defects may grow through one-by-one addition of atoms, in the end, it also depends on the diffusion of forming elements, Bonafos et al. (1998). Classification of these several-atom defects is normally done upon the nature of their constituents, size or shape. Clusters made of native point defects are normally classified as follows:

- Amorphous Pocket $(A P)$ : Also called as $I V$-Cluster, is the agglomeration of several $I$ s and $V$ s together into one single defect. Their behavior becomes important specially for damage accumulation through ion implantation.

- Voids: Or $V$-type Clusters, are the consequence of multiple reactions between vacancies, normally they appear into spherical shapes, which minimize the energy of the void.

- I -type Clusters: Accumulation of several Is into one coordinated cluster. Depending on the stages of the processing steps, their evolution can lead these defects to accommodate from irregular to more stable shapes and orientations.

Regarding this last type of defects, it is reported in studies for both $\mathrm{Si}$, Claverie et al. (2003) and Ge, Furuno et al. (1976) that $I$-type clusters, as they grow, can relax into rod-like shapes lying on the $\{311\}$ family of planes, (thus usually named $311 \mathrm{~s}$ or $113 \mathrm{~s}$, etc). For higher sizes, evolution leads to the so-called Dislocation Loops $(D L s)$, which 
are disk-shaped structures lying into $\{111\}$ and $\{001\}$ planes. Once again, these processes have been more studied in $\mathrm{Si}$, and known to be the cause of transient enhanced diffusion. In Ge there is still uncertainty on the behavior of such defects. Moreover, these type of defects cal also be produced while observing the samples by TEM through electron beam irradiation, adding difficulties to interpret the results, Claeys and Simoen $(\overline{2008})$. Recent studies, Hickey et al. (2008); Panciera et al. (2010); Boninelli et al. (2012a) show that it can be more likely to $I$-type clusters in Ge to lie in $\{111\}$ or $\{001\}$ planes with $D L$ shapes rather than in 311 structures.

Fig. 1.4 is a Cross TEM observation of $I$-type Extended Defects in Ge produced after implantation of $2 \times 10^{14} 100 \mathrm{keV}$ Ge ions and annealed at $340{ }^{\circ} \mathrm{C}$ for 1 hour, taken from Boninelli et al. (2012a). The inset in the left shows a high resolution image of an irregular small cluster while in the right, a $D L$ is shown.

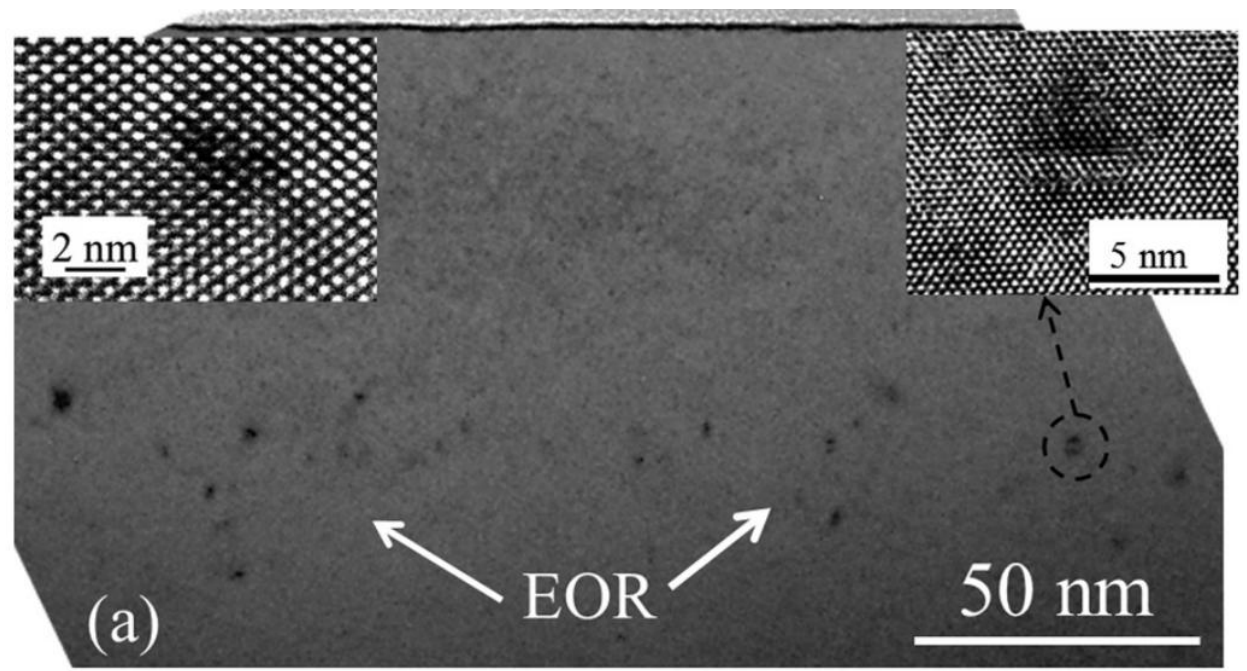

Figure 1.4: Cross TEM observation of $I$-type Extended Defects in Ge produced after implantation of $2 \times 10^{14} 100 \mathrm{keV}$ Ge ions and annealed at $340^{\circ} \mathrm{C}$ for 1 hour. The inset in the left shows a high resolution image of an irregular small cluster while in the right, a $D L$ is shown. After Boninelli et al. (2012a). 


\subsubsection{Damage accumulation}

It has already been introduced that ion implantation can generate defects through atomic collisions by the incoming, or secondary ions, within the target sample. As the ion fluence (quantity of implanted ions) increase, the number of displaced atoms out of their regular lattice positions, (and thus $I \mathrm{~s}$ and $V \mathrm{~s}$ ) increase. Through interactions between these fundamental defects, APs are formed and/or grown.

At low temperatures, (near liquid nitrogen temperature, $\mathrm{LN}_{2} \mathrm{~T}$, for example) it can be assumed that the mechanisms of damage generation and accumulation are due to mainly ion-target interactions, and reactions between sufficiently-close defects. For higher temperatures, the process starts to increase in complexity, and other mechanisms start to gain relevance, as migration events of $I \mathrm{~s}$ and $V \mathrm{~s}$, as well as $I V$-recombinations (as shown in Fig. 1.2(a) and Eq. 1.1) in the lattice or even inside APs, Pelaz et al. (2004).

In order to make predictive models which agree with experimental measurements, it will become important not to only understand the complex physical mechanisms involved in the experiments or processing steps, but also the actual measurement procedures and standard analysis performed in characterization techniques, allowing to a successful interpretation of results, and effective modeling.

Two group of techniques are normally used in order to see the damage accumulation. Transmission Electron Microscopy (TEM) and its variants, cross sectional TEM (XTEM), Plain View TEM PV-TEM or High Resolution TEM (HRTEM), allow to characterize the damage through optical investigation of the sample, where defects can be seen, and their density and size extracted from image analysis. Resolution of these techniques, which may in several cases depend upon the material properties, and substrate sizes and preparation, becomes important to understand what can, and cannot be seen. For instance, in Si, small defects after ion implantation can be detected,Claverie et al. (2003) while in Ge defects below certain size are invisible for existing TEM techniques, Boninelli et al. (2012a).

Rutherford Backscattering (RBS) techniques, specifically channeling RBS (c-RBS, or cRBS) technique, may be used to determine the degree of lattice distortion induced in the target by ion implantation to then, extract the quantity of damage produced after the process. This is performed through standards or calibration from the so-called "virgin", crystalline sample, to a fully random substrate measurement. Thus, the degrees of partially 

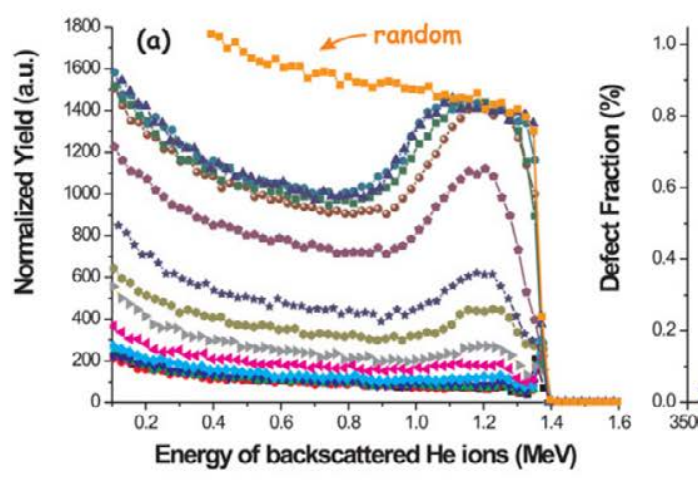

(b)
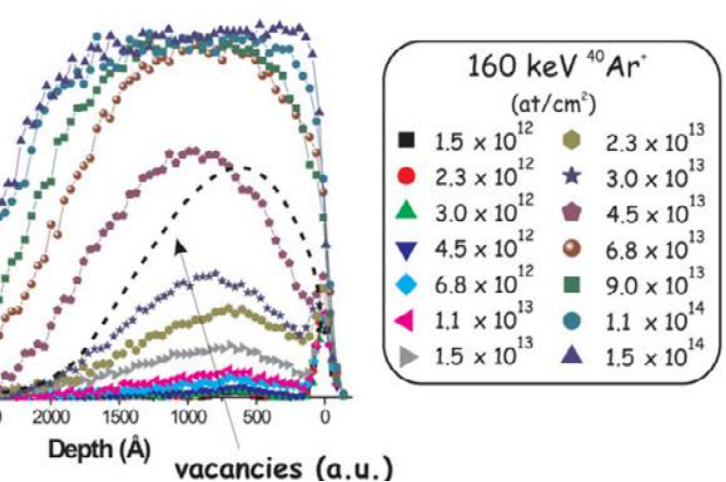

Figure 1.5: Different c-RBS measurements taken after implanting $160 \mathrm{keV}$ Ar ions at different fluences into Ge samples. (a) is the Normalized Yield and (b) is the defect fraction caused at every condition. After Decoster and Vantomme (2009).

randomized measurements, depending on the dose will indicate the degree of damage inside the sample, before amorphization, for which the measurement will be similar to the random standard. An example, taken from Decoster and Vantomme (2009), is shown in Fig. 1.5. where different c-RBS measurements are taken after implanting $160 \mathrm{keV}$ Ar ions at different fluences into different Ge samples. By comparing the Normalized Yield (a) of every sample to the random and virgin profiles, the defect fraction caused at every condition can be extracted (b).

\subsubsection{Amorphization}

Amorphization is a process of solid phase transition in which a crystalline material looses its structure into a disordered arrangement of atoms. In the limit of damage accumulation, for a high enough number of lattice atoms displacements, this process occurs. As in the pure damage accumulation case, the extension of fully amorphized layers depends on the ion mass, the fluence (normally expressed in ions per area of implantation), the temperature, and the flux (ions/time), since there is a quantity of damage needed to overcome in order to produce the change of phase.

Both damage accumulation and amorphization mechanisms have been widely studied 
in $\mathrm{Si}$, while recent studies in Ge have revealed uncertainties in the damage accumulation and amorphization mostly due to low mass ions, Koffel et al. (2009). In general, three differentiated damage accumulation regimes have been observed for both Si, Pelaz et al. (2004) and Ge, Decoster and Vantomme (2009) pointing out that the same fundamental mechanisms take place. These will occur for increasing ion dose, but again, will depend on the implantation conditions. If damage accumulation itself is a very complex process, amorphization adds a new degree of difficulty to make predictive models of damage generation through ion implantation.

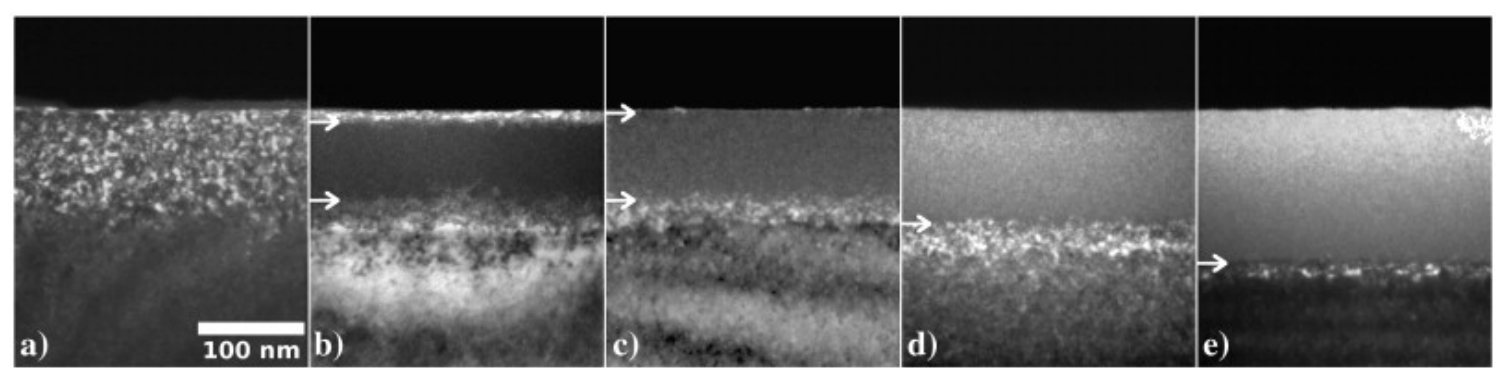

Figure 1.6: XTEM observation of the damage accumulation, amorphization and further extension of the amorphous layer caused by $150 \mathrm{keV}$ Ge ions implanted into Ge samples for increasing doses, from a) to e). After Claverie et al. (2010).

Fig. 1.6 is an XTEM observation of the damage accumulation, amorphization and further extension of the amorphous layer caused by $150 \mathrm{keV}$ Ge ions implanted into Ge samples for increasing doses, from a) to e), taken from Claverie et al. (2010). Arrows indicate the amorphous/crystalline $(\alpha / c)$ interfaces. In a) the damage in form of $A P$ s can be observed.

\subsubsection{Annealing}

Annealing procedures stand normally for heat treatments performed to produce different effects. Macroscopic changes in the material will appear under annealing, of different degrees of complexity. Thus, understanding the mechanisms at the point defect scale, can 
shed light on these effects. The increase of temperature adds energy to the system, activating diffusion of point defects, native or dopants, as well as breakup or emission reactions. Depending on the temperature and time of these heat treatments, Frenkel-pair recombination can occur, at different levels of magnitude but as well migration of point defects can end into formation of extended defects, which may be stable at the new conditions. Moreover, damage can be completely healed, by bulk or surface recombinations.

During ion implantation, the role that the temperature plays is normally related to the so-called dynamic annealing. The same quantity of the same ions implanted at the same energy can cause amorphization at low temperatures, but only a small amount of damage damage at high temperature conditions, Impellizzeri et al. (2009a). The same occurs depending on the time of implantation. Even at high temperatures of implantation, if the flux is high enough, the generated damage will not have enough time to recombine, or heal, leading to amorphization in conditions for which at a standard flux, no damage at all will effectively be measured, Posselt et al. (2006).

If full amorphous layers have been formed in the substrate, annealing at sufficiently high temperatures and times can cause the reverse phase transition through a SPER process.

\subsubsection{Solid Phase Epitaxial Regrowth}

A fully amorphized layer can recrystallize by following a heat treatment, through a layer-by-layer mechanism, starting from a crystalline seed, normally the amorphous $(\alpha)$ crystalline (c) interface, called Solid Phase Epitaxial Regrowth, or Recrystallization, (SPER). Different notations are also used in the literature, as Solid Phase Epitaxy (SPE), or Solid Phase Epitaxial Growth (SPEG).

This process occurs through a reorganization of the amorphous phase atoms into crystalline positions, beginning at the mentioned interface between the amorphous and crystalline phases, acting as a seed for recrystallization. During this heat treatment, different defects may appear due to different mechanisms. They are normally differentiated into two subgroups: regrowth-related defects, and end-of-range (EOR) defects, which will be discussed in the next section. Regrowth-related defects appear as a consequence of different lattice orientation fronts coinciding, causing a mismatch in the structure, thus provoking defects and dislocations which look like hairpins or of clamshell-type. It has also been 
reported that twin defects not only appear in this process, but can also play an important role for different SPER velocities, Martin-Bragado (2012).

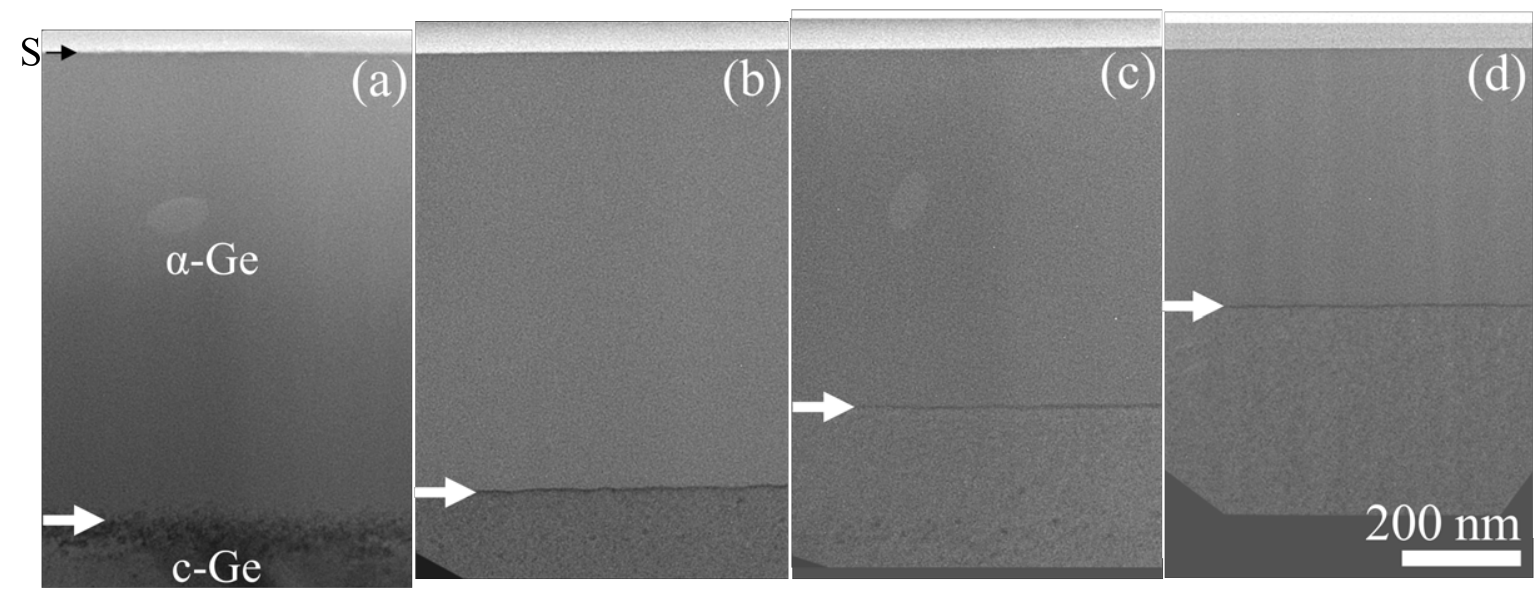

Figure 1.7: XTEM observation of Ge SPER process after $330^{\circ} \mathrm{C}$ anneal for different times, from (a) to (d). The $\alpha / \mathrm{c}$ interface is indicated with an arrow. After Darby et al. (2013).

As this is a thermally activated process, the velocity of regrowth depend on the temperature. This dependence is highly observed and even small variations of the local temperature affect the macroscopic evolution of the $\alpha / \mathrm{c}$ interface, Claverie et al. (2010). This observation has served as an explanation of the different reported growth rates. SPER also depends on the crystalline configuration of the $\alpha / c$ interface itself, and different rates have been reported depending on the substrate orientation in both Si, Csepregi et al. (1978) and Ge, Csepregi et al. (1977) materials. The regrowth-related defects also depend on the substrate orientation, being, for example, (100) epitaxially regrown layers less defective than (111) substrates. For Si, Ge and SiGe alloys, stress effects also affect the SPER process, and it becomes important to understand the dependence of regrowth upon hydrostatic pressure, tensile and/or compressive stress in any relevant orientation.

The process is represented as an example in Fig. 1.7, where a set of XTEM observations of the $\alpha / \mathrm{c}$ interface evolution over time in an amorphized Ge sample is shown. 


\subsubsection{End of Range Defects}

After amorphization of full continuous layers, there are damaged regions below the $\alpha / \mathrm{c}$ interface. After the SPER process, this damage evolves at the process temperature through very complex mechanisms that include point defect diffusion, Frenkel-pair recombinations and dissolution of small clusters, feeding bigger, and more stable extended defects. There is a point in which the size of these defects, and their location within the lattice, makes it more favorable to relax towards a more thermodynamically stable configurations, which lie on selected planes and shapes.

The process underlying growth and dissolution of these defects is normally referred as Ostwald ripening. In a few words, there is an effective flux of point defects between small, less stable, clusters and big, more stable, ones. This flux, provides a supersaturation of point defects, when compared to the equilibrium concentration of native point defects ( $I$ -type, for example). This process makes the bigger clusters grow at expenses of the small ones, thus, decreasing the density of defects within the sample, but increasing the size of those defects, until the point defect reservoir which produced the supersaturation is emptied, and the equilibrium is broken. At that point, damage is dissolved for increasing times. These very complex mechanisms depend strongly on the initial damage, its distance to the surface, the annealing temperature and time, and the recombination capability of the surface, as it has been widely studied in Si Cowern et al. (1999). On the contrary, in Ge this process has only been observed for temperatures below $600^{\circ} \mathrm{C}$. Fig 1.8 is a set of Plain View TEM (PVTEM) images of the defects caused by ion implantation in Ge of 15 $\mathrm{keV} 1 \times 10^{15} \mathrm{P} / \mathrm{cm}^{2}$ and their thermal evolution for subsequent anneals at $500{ }^{\circ} \mathrm{C}$ where this process can be observed: a) $10 \mathrm{~s}$, b) $30 \mathrm{~s}$ and c) $60 \mathrm{~s}$, taken from Claverie et al. (2010). For temperatures higher than the mentioned $600^{\circ} \mathrm{C}$, the process became non-conservative leading to a dissolution of the damage by decreasing both size and number of defects.

As it has been pointed out, native point defects play a crucial role on dopant diffusion. Thus, the EOR defects, and their behavior under process temperatures, will strongly affect the dopant profiles and their activation, and in the end, the electrical properties of the future device. In Ge, already exposed uncertainties upon point defects and, of course, EOR defects themselves, make it difficult to understand the behavior and evolution of these defects Claverie et al. (2010); Koffel et al. (2009). Nevertheless, recent studies shed 

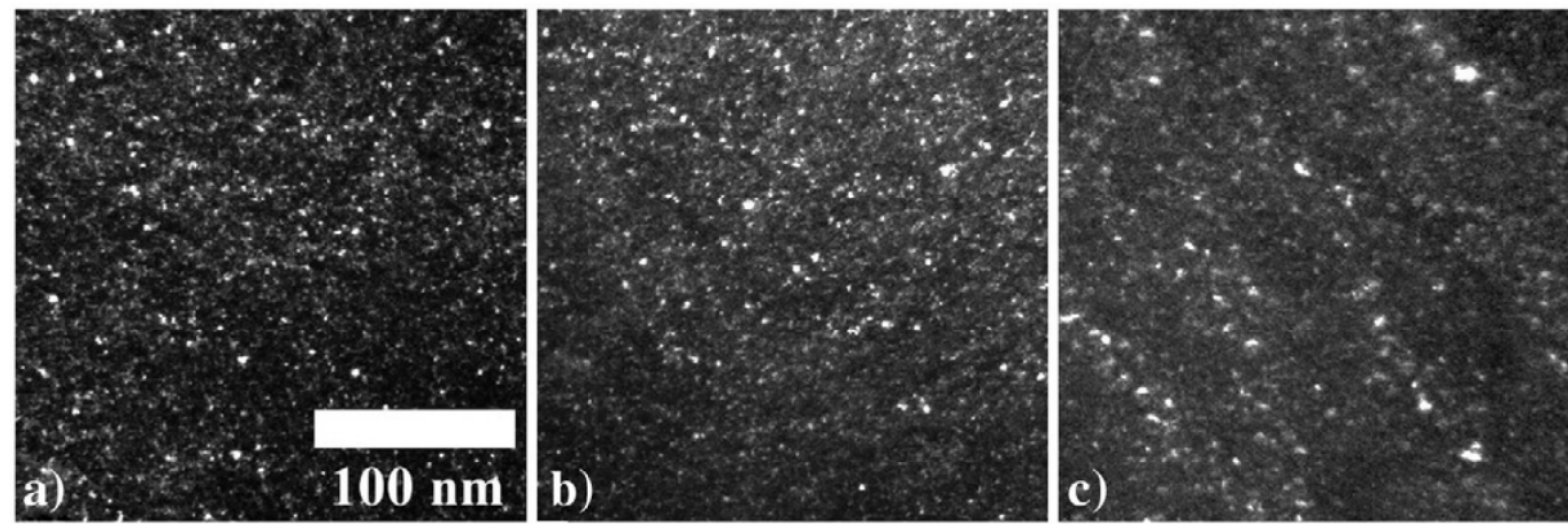

Figure 1.8: Set of PVTEM images of the defects caused by ion implantation in Ge and their thermal evolution for subsequent anneals: a), b) and c), taken from Claverie et al. (2010).

light on their role in B deactivation, Panciera et al. (2010) or the influence produced by the distance to the surface in their dissolution, Boninelli et al. (2012a).

\subsubsection{Why Kinetic Monte Carlo?}

Depending on the experiment that is wanted to reproduce, the simulation technique has to be chosen attending to different factors. Usually, a compromise has to be found between the level of detail needed to reproduce or predict a certain effect, and the computational costs in terms of time and resources. It is always desired to use models which take into account the maximum number of possible effects, but reducing the simulation times and expended resources.

Fig. 1.9 is a diagram of the most used techniques in materials science. Different simulation techniques are represented and arranged depending on the time and space scale which are usually capable of reproduce (x-axis), and the quantity of approximations, inverse to the level of detail achieved (y-axes).

Density Functional Theory (DFT), Dreizler and Engel (2011) uses a quantum mechanical approach to model the electronic structure of many-body systems, in particular atoms, molecules, and the condensed phases. It is based in solving the Schrödinger equation with 


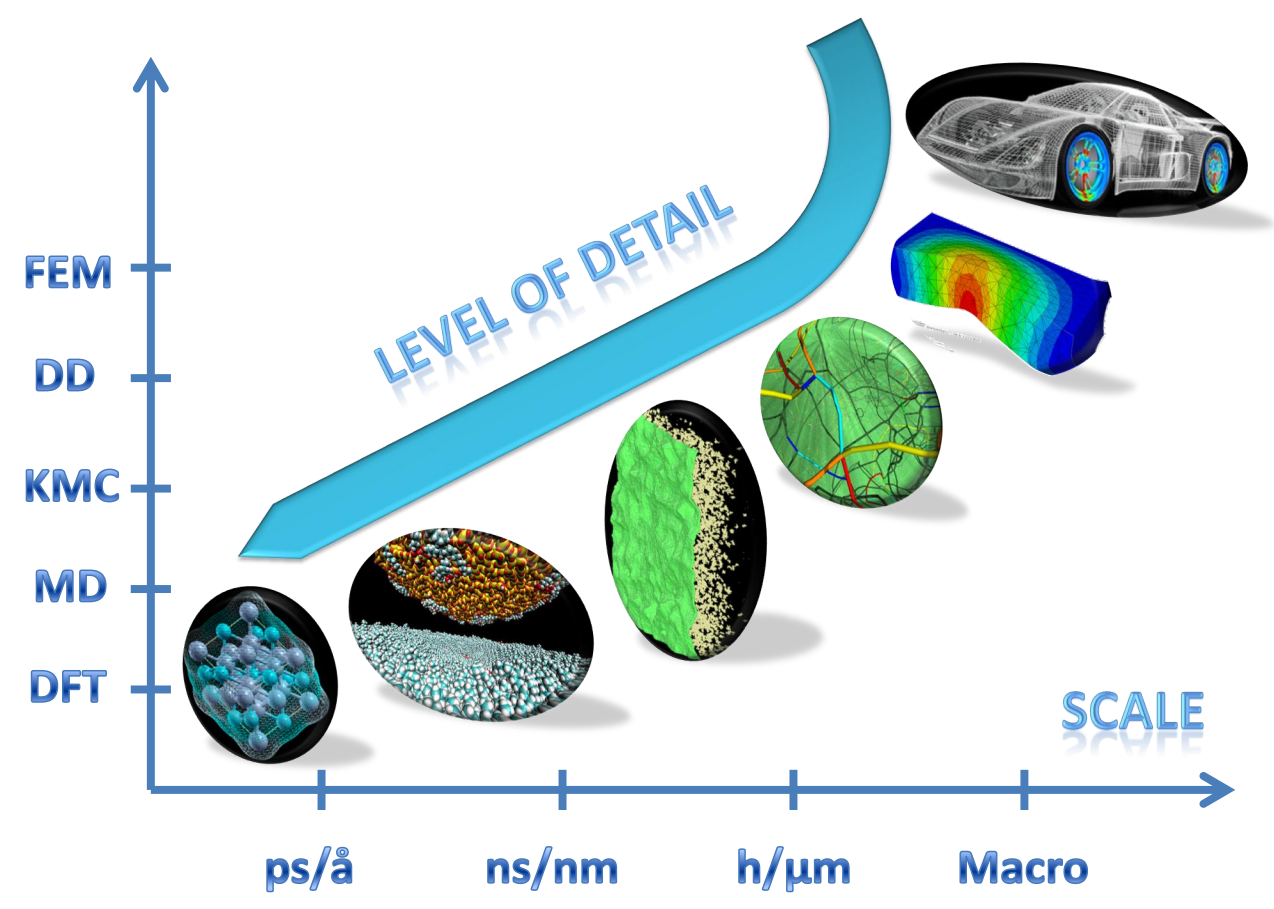

Figure 1.9: Indicative diagram of the simulation techniques used in materials science. Different simulation techniques are represented and arranged depending on the time and space scale which are usually capable of reproduce (x-axis), and the quantity of approximations, inverse to the level of detail achieved (y-axes). From the smallest scale to the biggest, Density Functional Theory (DFT), Molecular Dynamics (MD), Kinetic Monte Carlo (KMC), Dislocation Dynamics (DD), and the Finite Element Method (FEM) are represented by schematic representations.

few approximations, and the scales of simulation achieved are around a few $\AA$, and ps due to the extremely high number of calculations needed for every step. Molecular Dynamics (MD), Haile (1992) basically solves the newtonian equations for a given potential. Thus, the models and results are as good as the potentials used, which depend on all the materials present in the simulation. The scales are expanded from DFT, being able to simulate in the scale of ns, and up to a few $\mathrm{nm}$, being able to simulate atomic vibrations. Kinetic Monte Carlo (KMC) techniques, Landau and Binder (2000) use frequency rates, which depend on activation energies, normally extracted from DFT or MD parameteriza- 
tions, or experimental measurements. The main disadvantage of this method is the high number of parameters needed to perform realistic simulations. KMC allows to simulate up to hundreds of nanometers and several hours, depending on the number of objects considered. Normally defects at the atomic scale are simulated. Dislocation Dynamics (DD) techniques,Zbib et al. (1998) add another degree of abstraction, simulating bigger objects at the meso-scale, linking both the micro- and macro scales, but loosing some of the atomistic scale considerations. As indicated by the name, it is normally used to predict behavior of dislocation-sized defects at a micrometer scale, in a timescale similar to KMC. In Finite Element Modeling (FEM), Spyrakos (1994) all defect and atomic information is lost, and numerical techniques are used for finding approximate solutions to boundary value problems for partial differential equations. This is achieved by dividing the space into small parts with certain properties. Depending on the subdivision, very large scales may be achieved. The results that are obtained with these techniques, normally refer to macroscopic effects.

Of course, depending on the specific conditions, and the quantity of simulated objects or information, constrains of space/time scales may highly vary in all simulation techniques.

As an example, in the topic concerned by this dissertation, DFT can be used to obtain the self-diffusion constants in Si, Blöchl et al. (1993) MD to understand the different behavior between concentrated and diluted damage in Si, Pelaz et al. (2004) DD to investigate the mesoscopic plastic deformation in Si crystals, Ghoniem et al. (2000) and FEM to model physical effects in transistors at the device operation scales, Buturla et al. (1981).

In our case, we are interested into understand the atomistic mechanisms of defects induced by materials processing techniques. This is performed in the scale of hundreds of nanometers, at times that span from a fractions of seconds (between ions implanted in the sample) and long annealing times, i. e. hours. Thus, KMC appears as the tool of choice that also allows to conserve the atomistic scale relevant information.

\subsubsection{Summary}

In this chapter, the state-of-the-art of the different semiconductor processing techniques has been generally presented and reviewed, focusing in the the knowledge achieved up to date for the particular case of Ge. After introducing a justified motivation on the 
topics covered in this dissertation, and establishing the objectives of the work, background information has been given by defining basic concepts and explaining the processes that will be discussed. Background information on doping semiconductor techniques, defects (point defects and extended defects), amorphization and recrystallization processes has been given, providing the general basics needed to follow this work. Different simulation techniques have also been presented and KMC has been justified as the most convenient approach to be followed in order to achieve the pursued objectives.

The complexity in the mechanisms underlying damage accumulation and dissolution, amorphization and SPER in Ge can now be anticipated. Which role will the temperature, fluence, flux and ion mass, for example, play in this process? How existing techniques can extract the information for what happened within the sample while performing the stages of front-end processing? Can we apply the same well-known modeling techniques in the area of semiconductor processing to Ge? Which and how models and simulation techniques are able to reproduce and explain these experiments? This thesis will try to shed light upon these questions.

\subsection{Organization of the Dissertation}

After the introduction and provided in this Chapter, this thesis is divided in two main Parts.

First, in Part I the simulation methods used and developed during this work are explained. In Chapter 2 the Kinetic Monte Carlo method, and its implementation into MMonCa, an Object Kinetic Monte Carlo simulator for damage irradiation evolution and defect diffusion, is presented, discussed and validated against both theoretical and experimental results. In Chapter 3 the Lattice Kinetic Monte Carlo module is presented and also validated through comparisons with Si SPER experimental results. Finally, Chapter 4 explains generally the Binary Collision Approximation simulation technique used in this work.

Part II comprises the main results achieved in this thesis. Chapter 5 deals with the OKMC modeling of the mechanisms of damage accumulation and amorphization in Ge caused by ion implantation. In Chapter 6, the LKMC model for SPER in Ge of amorphized 
layers, and its dependence on the substrate orientation are discussed. A simple calibration based on the SPER rates on the applied hydrostatic pressure is also discussed. As explained in both Chapters 5 and 6 , the coupling of both OKMC and LKMC models into the simulator provides a useful tool for further modeling of the full amorphization and SPER process.

Lastly, Chapter 7 summarizes all conclusions and results achieved in this work, and propositions of further investigations are provided in Chapter 8 . 


\section{Part I: Methods}

"Computers are useless. They can only give you answers."

- Pablo Picasso 


\section{ObJect Kinetic Monte Carlo}

In this chapter, the Kinetic Monte Carlo (KMC) algorithm is presented along with the physicaly-based Object KMC (OKMC) simulation technique, and its implementation into the Modular Monte Carlo Simulator, MMonca. First, it will be described how this technique is able to simulate the time evolution of some processes at a given known rate, following by how the objects (defects) are placed into the simulation space, and the events that these objects can perform. Finally, it will be used to reproduce simulated results for defect evolution in silicon using the presented simulator, and comparisons with available experimental data will be given, along with theoretical validations. These comparisons are used to validate MMonCa, showing its versatility to be used for modeling the behavior of defects in different materials. Such implementation and validation for damage irradiation evolution and defect diffusion in different materials has already been published in MartinBragado et al. (2013).

\subsection{Introduction}

In Chapter 1 the importance and the different effects produced by ion implantation in semiconductors, along with the simulation techniques normally used for modeling those processes were introduced. These approaches, in the form of simulation tools, are widely used and count with codes that are robust and flexible enough to allow for deep research 
in a wide range of materials. For instance, SIESTA, Soler et al. (2002), VASP, Kresse and Hafner (1993) or Gaussian, Frisch et al. (2009) provide solutions for first principle calculations. LAMMPS, Plimpton (1995) GROMACS, Berendsen et al. (1995) and many others are used in the area of Molecular Dynamics, and there are many packages to run continuum (finite element method) simulations of which Abaqus and Ansys simulators, to name just a few, are well known and established.

For KMC, some existing codes are DADOS, Jaraiz (2004) for diffusion of defects in silicon based materials, BIGMAC, Johnson et al. (1998) initially designed for silicon, Sentaurus Process KMC, a commercial software for Si based materials, Sentaurus (2012); Martin-Bragado et al. (2006) and LAKIMOCA, Domain et al. (2004) a Lattice KMC (LKMC) code used for simulation of irradiated metals. Nevertheless, a multi-material oriented, easy to access code for performing KMC simulations does not seem to be clearly established. This lack could be negligible would it not have been for the extreme usefulness played by KMC simulations in the field of damage irradiation. Being in the border between atomistic and continuum simulation, KMC plays a very important role in using all the theoretical information on activation energies obtained by the previously cited methods, and connecting them to macroscopic experiments, Fu et al. (2005); Castrillo et al. (2001). Thus, regarding the ITRS requirements for Modeling \& Simulation exposed in Chapter 1, an effort has been made to develop a KMC simulator, written in Object Oriented Programming (OOP) $\mathrm{C}++$ and integrated with a TCL language user interface, aiming to be multi-material, powerful, flexible and easy to use, filling the need for this type of codes that exist in the field of Monte Carlo simulation, Amar (2006).

This Chapter is structured as follows: KMC theory is presented in Section 2.2 and its implementation into the developed code, MMonCa, is discussed in Section 2.3, where the major modules, time (2.3.1) and space 2.3.2), are reviewed. After, OKMC is presented as the particular consideration of atomistic defects as the objects to be simulated in Section 2.3.3. Some examples of the results achievable with the presented code using OKMC simulations are written in Section 2.4. for validation against both theoretical 2.4.1) and experimental results 2.4 .2 on particle diffusion and damage dissolution in $\mathrm{Si}$, respectively. 


\subsection{Kinetic Monte Carlo}

The goal of the Kinetic Monte Carlo algorithm is to simulate the dynamic evolution of a system which might be out of the equilibrium, Bortz et al. (1975); Voter (2007); Landau and Binder (2000). Such system is considered to be formed by different objects which can rely on different states. Transitions between these states are assumed to be Markovian, i.e. the transition rates $\nu_{i j}$ depend only on the initial, $i$, and the final, $j$, states. Such transitions are also assumed to be time independent. The input parameters of the algorithm are exactly those rates, $\nu_{i j}$, which cannot be predicted by the KMC method itself, but have to be extracted from fundamental experiments and/or other simulation techniques such as MD o DFT. In this work, $\nu_{i j}$ are modeled assuming the Harmonic Transition State Theory, Vineyard (1957) as Arrhenius equations with an activation barrier $E_{i \rightarrow j}$ (higher than $k_{B} T$ for this approach) and a prefactor $K_{i \rightarrow j}$ :

$$
\nu_{i \rightarrow j}=K_{i \rightarrow j} \times \exp \left(-\frac{E_{i \rightarrow j}}{k_{B} T}\right) .
$$

Fig. 2.1 is an schematic energy diagram which describes the physical meaning of the $E_{i \rightarrow j}$ activation barriers. For a simulated object in the system located in a steady state $i^{*}$, with an energy of $E_{i}^{f}$, a second steady state, $j^{*}$, at the energy level $E_{j}^{f}$ can be achieved after overcoming the transition barrier $E_{i \rightarrow j}=E_{j}^{f}-E_{i}^{f}+E_{i j}^{b}$. The opposite $E_{j \rightarrow i}$ would only be the barrier $E_{j \rightarrow i}=E_{i j}^{b}$. Assuming that the concentration of particles in the $i$ state is $[i]$ and in the $j$ state is $[j]$, the steady state is reached when $[i] \times \nu_{i \rightarrow j}=[j] \times \nu_{j \rightarrow i}$. Using this notation (see Fig. 2.1) and assuming that $K_{i \rightarrow j}=K_{j \rightarrow i}$, the equilibrium concentration ratio between states $j$ and $i$ is then given by:

$$
\frac{\left[j^{*}\right]}{\left[i^{*}\right]}=\exp \left(-\frac{E_{j}^{f}-E_{i}^{f}}{k_{B} T}\right) .
$$

Thus, this relation does not depend on the activation barrier, but only on the energy levels (or formation energies) of both states. Generalizing for a high number of objects and states, out of steady state, with KMC, the dynamic behavior of the system can be simulated, providing a way to take time evolution into account.

Once all transition rates, $\nu_{i \rightarrow j}$, for all possible states in the system are known (that is, they are given as input parameters) the KMC algorithm starts. For simplicity, the initial 


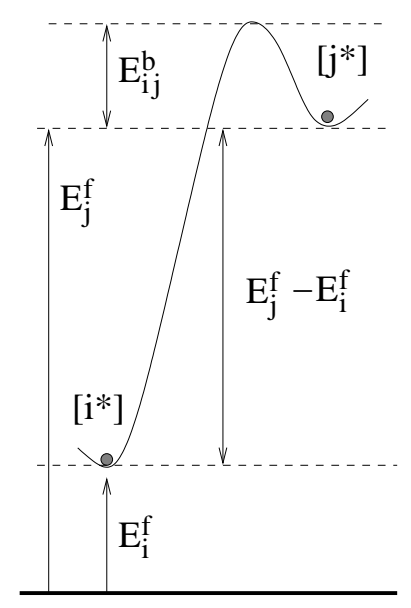

Figure 2.1: Energetic diagram for a transition between two states: $i$ and $j$. Formation and barrier energies are represented. After Martin-Bragado et al. (2013)

state in the transition rates is omitted. Thus, the rate $\nu_{i \rightarrow j}$ will now be written as $\nu_{j}$, being $j$ the final state achievable from a particular initial state $i . K_{i \rightarrow j}$ will also be written as $\nu_{0}$. Using this notation, the KMC direct method, Gillespie (1976) is applied as follows:

1. Obtain the cumulative functions

$$
R_{i}=\sum_{j=1}^{i} \nu_{j}
$$

for $i=1, \cdots, N$. Being $N$ the total number of transitions in the given system.

2. Compute two random numbers, $r$ and $s$ in the interval $(0,1]$.

3. Find $i$, the event to perform, for which $R_{i-1}<r R_{N} \leq R_{i}$.

4. Perform the event $i$ : transform the particular chosen object from $i$ to $j$.

5. Increase the total simulated time by

$$
\Delta t=\frac{\ln (1 / s)}{R_{N}}
$$

6. Recalculate the affected rates. 
7. Return to step 1 until the requested physical time has been simulated.

This standard KMC algorithm takes only care of the time evolution. Space dependence is intrinsic to the proper definition of each event. In our case the presence of physical defects that diffuse in space implies the need to include diffusion as a transition rate, and to define algorithms for space migration and particle interaction. Consequently, in MMonCa, the main modules are:

- Objects and the list of their associated transition rates and actions.

- A rate manager to compute the time evolution and to select the event to perform.

- A space manager to manipulate space translations, neighbor search and defect interactions.

After these general definitions, and for the sake of simplicity, this chapter will only focus on the Object-KMC (OKMC) method. Here, the objects simulated are defects as point defects or clusters (see Section 2.3.3). The same assumptions and general definitions of rates and states will be used for LKMC, where the objects (and of course the states) are different. Such objects will be lattice atoms lying on the amorphous/crystalline interfaces, as described in Chapter 3 .

\subsection{MMonCa}

Fig. 2.2 shows the overall structure of MMonCa. The main code is written in $\mathrm{C}++$ (in red) with implemented extensions of TCL, allowing to use an already existing and well known language in the input scripts and to implement a user interface, instead of a selfcreated language, increasing the code versatility. The commands that have been extended allow the user to define a simulation cell, the 3D definition of the material structure of the simulation, reading of damage from external files, annealing the damage, reading and writing the input parameters that the simulations need, and output of different calculated magnitudes generated during the simulation. The rest of modules are described below. 


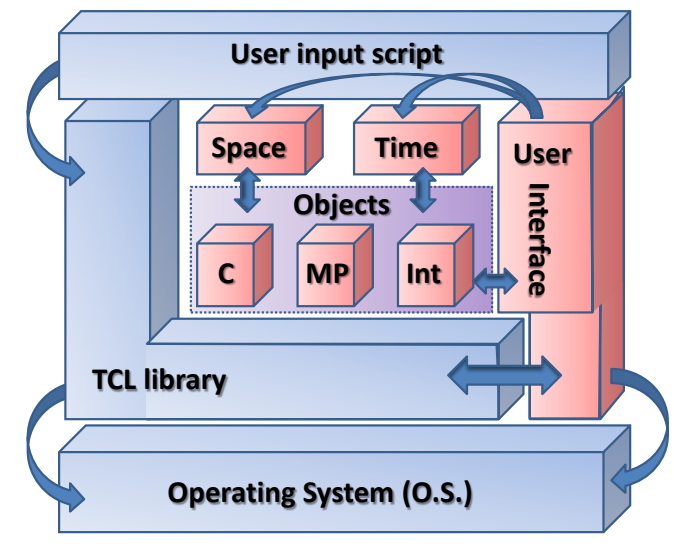

Figure 2.2: Overall structure of MMonCa. The user interface relies on a layer of the TCL interpreter, extended to support the simulator. The extension relies on specialized modules to control the space, time and defects. Several defects are supported as the objects to be simulated: clusters (C), mobile particles (MP) and interfaces (Int).

\subsubsection{Rates and time handling}

Fig. 2.3 shows graphically the idea behind the event selection involved in step 3 of the KMC algorithm previously explained. Once an updated list of all the transitions associated with the objects being simulated is generated, one of them is chosen proportionally to such rates. The $\Delta t=-\ln (s) / R_{N}$ associated with the simulation of such event is independent on the event chosen, depending only on the whole system. In practice, iterating through all the cumulative rates to find the one to be performed is not efficient when there is a large number of rates. For this reason, the simulator does not contain a transition bar with all the rates, but rather a binary tree, where the access time to each rate is not proportional to the number of them, $N$, but to $\log _{2}(N)$. In the one hand, this improves the access time to the chosen event, on the other hand, the binary tree degrades the insertion, deletion and modification time for rate insertion from a constant time to also $\propto \log _{2}(N)$ time. Overall, the balance is positive when there are a large number of rates in the system and more selection of rates than insertions, modifications or deletions. 


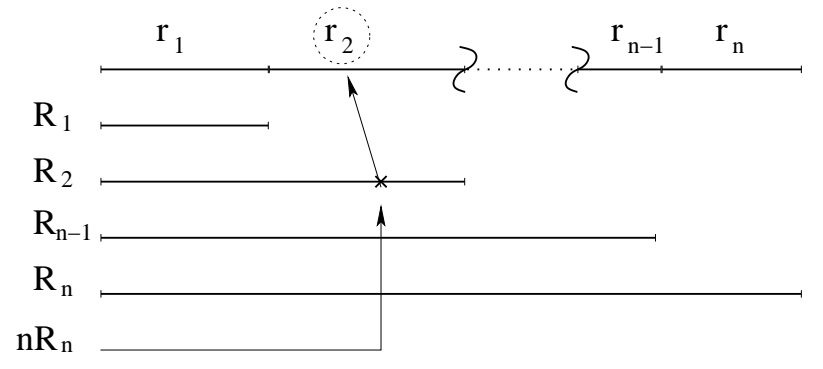

Figure 2.3: The KMC algorithm contains a list of all the transitions associated with the objects being simulated, and picks the next one proportionally to such rates. That can be seen graphically as getting a random number uniformly distributed in $\left[0, R_{N}\right)$ and picking up the event "aligned" with such number. After Martin-Bragado et al. (2013).

\subsubsection{Space handling}

The space is divided into small, variable along $x, y$ and $z$ axes, prismatic elements using a tensor mesh. Space is assumed to be homogeneous (material, temperature and other fields) inside each small element. Each element obtains its material definition by calling a user-defined procedure that allows the specification of the material structure in the simulation. This way, very complex shapes containing different materials can be simulated. When two consecutive mesh elements have different materials, an interface object, as shown in Fig. 2.4 a), is built between them. In this space implementation, for now, all elements are of the same size, and no mesh-refinement algorithms are supported.

Efficient neighbor search is implemented by having a standard link cell method, Hockney and Eastwood (1988). Once the list of neighbors is obtained a look-up table is used to implement user-defined allowed interactions.

The capture distance $r_{c}$, shown in Fig. 2.4 b), must be provided for every single particle. It is typically of the same order as $\lambda$, the microscopic migration distance. In our simulator, any non point defect (except interfaces) is created by the agglomeration and tracking of its constituent particles. This implies naturally that the capture distance of any defect is the overlay of all the capture distances of all the defect constituent particles as seen in Fig. 2.4.). It also means that clusters can have any shape and a capture volume that will adapt to it as long as the particles are configured to form such shape. 


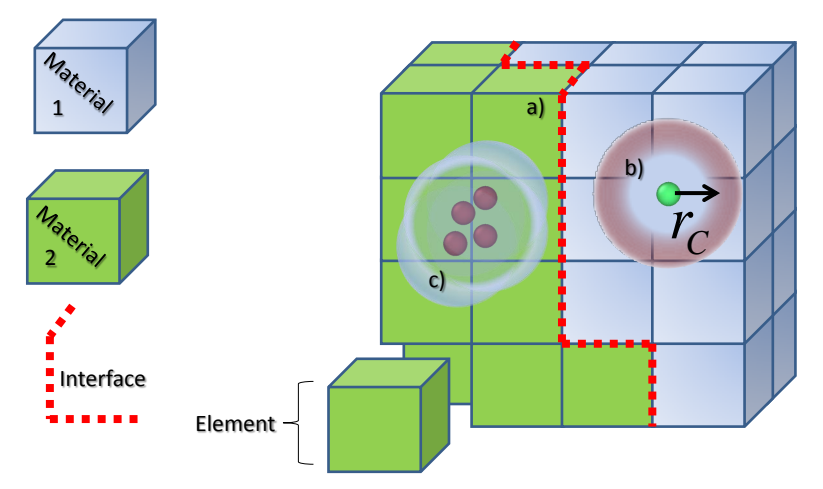

Figure 2.4: Space is divided into small prismatic elements using a tensor mesh. a) An interface is the union of all element faces between adjacent different materials (2D objects). b) The capture distance $r_{c}$ of every particle, which leads to a 3D capture volume, is defined independently. c) The capture distance of clusters is built as the union of the capture distances of their constituent particles.

\subsubsection{OKMC: Defects}

The last part needed to be defined are the objects to be simulated. Fig. 2.5 is a scheme of the objects simulated by OKMC. A high magnification image from a XTEM micrograph of a dislocation loop formed in a Ge sample implanted with $30 \mathrm{keV} 2 \times 10^{14} \mathrm{Ge} / \mathrm{cm}^{2}$ after annealing at $340 \mathrm{C}$ for $1 \mathrm{~h}$ is presented in a) (after Boninelli et al. (2012a)). In b) the objects that are to be simulated are highlighted. In this case, Is (red) and Vs (green) as mobile particles, and the dislocation loop as a cluster formed by $I$ s . Once known the rates of the possible events to be performed by every object, assuming that the lattice influence in the defect behavior is included in such rates, OKMC takes only care of the defects, getting rid of simulating the lattice c). From this example, coupled with the theory exposed in 2.2, the nature of OKMC can now be better understood. The evolution over time and space of the defects and dopants introduced in the semiconductor sample, by means of ion implantation, (see Sec. 1.3.3 will be the simulated objects through some rates that will be different for every event, or transition between states.

Depending on the type of object, the simulated events may be very different. Thus, 
a)

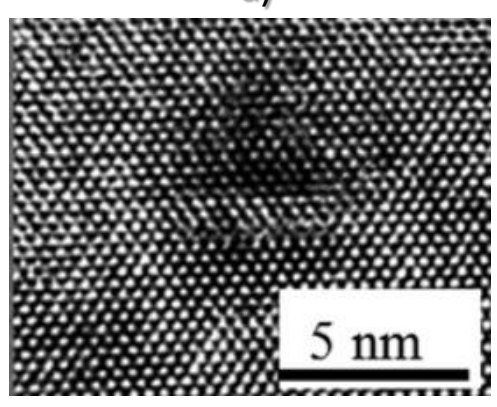

b)

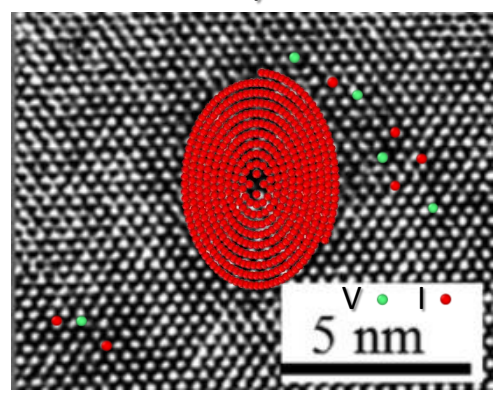

c)

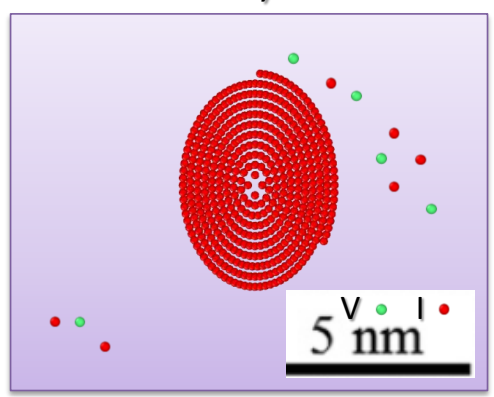

Figure 2.5: Example of the objects to be simulated by OKMC. a) High magnification image from a XTEM micrograph of a dislocation loop formed in a Ge sample implanted with $30 \mathrm{keV} 2 \times 10^{14} \mathrm{Ge} / \mathrm{cm}^{2}$ after annealing at $340 \mathrm{C}$ for $1 \mathrm{~h}$ (after Boninelli et al. (2012a)). In b) the objects that are to be simulated are highlighted. In this case, Is (red) and $V \mathrm{~s}$ (green) as mobile particles, and the dislocation loop as a cluster formed by $I \mathrm{~s}$. Assuming that the lattice influence in the defect behavior is included the rates, OKMC takes only care of the defects, getting rid of simulating the lattice c).

the events will be defined for each defect. In particular, such defects are classified as: interfaces (Int), mobile particles (MP), and clusters (C) (See Fig. 2.4). The properties we simulate for each of them are described next. As they are considered objects of the OKMC simulator, they are treated in a similar way. Each object, to be included in the simulator, needs to have the following data and functions defined (with the notation already defined in Sec. 1.3.3):

- Number of events associated with the object. For instance, three for MPs: diffusion, breaking-up and creation of Frenkel pairs $\left(A \rightarrow A+I+V \rightarrow A_{i(V)}+V(I)\right)$ to react with impurity atoms.

- Rate associated to each event. In this example for MPs, computing the diffusion, breaking-up and injection rates, or returning zero if they do not apply.

- Functions to perform each event when it is chosen by the OKMC algorithm. For example, a MP needs the implementation to move the particle, break it up or create and/or react with Frenkel pairs. 
Some of the explained events (break up and creation/reaction with Frenkel pairs) implement reactions similar to $A B \rightarrow A+B$. The forward reaction $A+B \rightarrow A B$ is implemented through diffusion. For this forward reaction to happen two things are needed: a) $A$ moving to the neighborhood of $B$, or $B$ into $A$, and $\mathrm{b}$ ) the reaction being allowed. Diffusion is implemented as an event for all defects but interfaces. At the end of such event, a look for neighbors is performed to detect potential reacting species as explained in Sec. 2.3.2. To properly react with such species, two more algorithms are needed in each KMC object:

- A look-up table that establishes whether the reaction is possible or not (taking into account possible reaction barriers)

- A function that implements the interaction itself, taking the reactants and transforming them in the result.

Finally, since during reactions the reactants are destroyed and the result is created, each object requires a constructor and a destructor that is able to properly build and erase respectively the objects from the KMC simulator.

\section{Int: Interface}

Fig. 2.4 shows how the plane between two different materials or a material and the outside world is defined using an interfaces object (red dashed lines). As explained in Sec. 1.3.3. interfaces can create and inject $\operatorname{MPs}\left(I_{\mathrm{s}}\right.$ and $V \mathrm{~s}$, and also impurities that were previously trapped). These emissions can be done to either side, assuming the MP may exist there. In the particular case of impurity emission, the model implemented corresponds to a three phase segregation model. Such model is shown in Fig. 2.6, taken from MartinBragado et al. (2013). MP impurities can be at the interface by overcoming the barrier to reach the interface $\left(E_{\text {barrier }}+E_{m}\right)$. Then, they have a rate $\nu=\nu_{0} \exp \left(-E_{\text {emit }}(\right.$ side $\left.) / k_{B} T\right)$ to be emitted to either side. $E_{\text {emit }}$ is set as $E_{b}+E_{\text {barrier }}+E_{m}$. Similarly to equation 2.2 , it is easy to see that the segregation coefficient, defined as the ratio between the concentration of particles at both sides at equilibrium, is

$$
S=\exp \left(\left(E_{b}(1)-E_{b}(2)\right) / k_{B} T\right)
$$




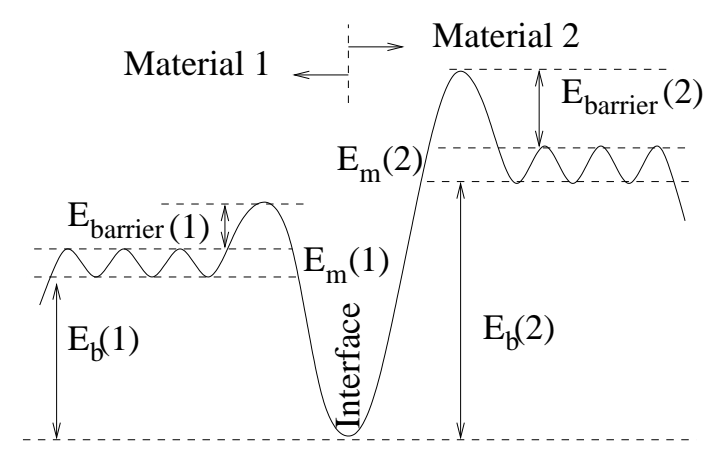

Figure 2.6: Three phase segregation model. Particles can be captured and emitted at either side, but the binding energies, migration energies and capture barriers might be different at each side. After Martin-Bragado et al. (2013).

When any diffusing defect arrives at the interface it can be annihilated according to certain probability set by the user. This applies to MPs and Cs.

\section{MP: Mobile particle}

Single $(H, I, V)$ or paired defects $(A s V, B I)$ are defined as MP in our simulator, where paired interstitial defects are assumed to be the same as impurities in the interstitial position $\left(B_{i}=B I\right)$, Fahey et al. (1989). The transitions associated with these MP objects are:

1. Migration, by simulating the random walk of small diffusion events with fixed migration distance $\lambda$ in one of the three perpendicular axes of the system, randomly chosen for each jump. The migration rate for mobile particles is computed as

$$
\nu=\nu_{0} \exp \left(-E_{m} / k_{B} T\right)
$$

where $\nu_{0}$ and $E_{m}$ are the input parameters for microscopic diffusivity.

2. Break up of a pair (or kick off mechanism) of $I$ or $V$ impurities. For instance, $A s V \rightarrow A s+V$. The break-up frequency equals

$$
\nu=\nu_{0} \exp \left(-E_{b k} / k_{B} T\right),
$$

with $E_{b k}$ being the activation energy for break-up. Such activation is computed as binding energy plus migration energy of the emitted particle. 
3. Injection of extra $I \mathrm{~s}$ or $V \mathrm{~s}$ by creating an $I V$ pair, capturing the $I$ or the $V$ and emitting the other (also called Franck-Turnbull mechanism). This reaction applies for instance to $B$ substitutional in $\mathrm{Si}: B_{s} \rightarrow B_{i}+V$. Its rate is modeled as

$$
\nu=\nu_{0} \exp \left(-E_{F T} / k_{B} T\right)
$$

where the activation energy for such example would be set as $E_{f}(I)+E_{f}(V)-$ $E_{b}\left(B_{i}\right)+E_{m}(I)$, being $E_{f}$ the formation energy.

MPs can interact with each other to form more complex defect objects: for instance $I+I, B_{i}+B$ or $I+$ Cs producing Cs.

\section{C: Cluster}

Cs are the agglomeration of several impurities and/or $I$ s or $V$ s. These objects can emit their constituent particles, transform into other Cs, migrate and trap/detrap impurities that might stop their diffusion. Cs can adopt different shapes to adapt to the realistic morphology of big defects in different materials. In particular, they can be defined as a) planes (similar to $\{311\}$ defects Martin-Bragado et al. (2008)), b) disks (similar to dislocation loops Martin-Bragado et al. (2008)), c) spheres (voids Estreicher et al. (1997)) and d) irregular clusters (no special shape). They can play different roles in the physical systems under consideration. In semiconductors, clusters of dopants with $I$ s and $V$ s deactivate partially the implanted dopants by forming agglomerations like $A s_{4} V$ Mueller et al. (2003); Pinacho et al. (2005) or boron interstitial clusters Pichler (2004); Pelaz et al. (1997).

The different events that Cs can perform are:

1. Emission of their constituent particles as MPs. The activation energy for emission is computed as the formation energy difference between the final and the initial state when positive, plus the migration energy of the emitted particle. The potential energies of all the clusters are required as input parameters for the simulation.

2. Emission of constituent particles in pairs. For instance, $A_{n} V_{m} \rightarrow A_{n-1} V_{m-1}+A V$. The activation energy being $E_{f}\left(A_{n-1} V_{m-1}\right)+E_{f}(A V)-E_{f}\left(A_{n} V_{m}\right)$ when positive, plus $E_{m}(A V)$. 
3. Injection of non-existing $I$ s or $V$ s by Frenkel pair creation $\left(A_{n} I_{m} \rightarrow A_{n} I_{m+1}+V\right.$ or $\left.A_{n} V_{m} \rightarrow A_{n} V_{m+1}\right)$. The activation energy equals to $E_{f}\left(A_{n} I_{m+1}\right)+E_{f}(I)+E_{f}(V)-$ $E_{f}\left(A_{n} I_{m}\right)$ when such value is positive (zero otherwise), plus $E_{m}(I)$ for injection of Is.

4. Migration. The migration rates are defined in a similar way to all the other migrations as

$$
\nu_{0} \exp \left(-E_{m}(\text { cluster }) / k_{B} T\right)
$$

5. Transformation. For transformation into other Cs,

$$
\nu=\nu_{0} \exp \left(-E_{\text {transform }}(\text { size }) / k_{B} T\right)
$$

being each transformation activation energy and prefactor defined by the user.

The number of different MP emission mechanisms for a simple $\mathrm{C}$ can be high. For instance, $B_{4} I$ clusters can make transitions to $B_{4}+I, B_{3}+B i, B_{3} I+B, B_{4} I_{2}+I$ and $B_{3} I_{2}+B V$. For clusters breaking into elemental particles (MPs) the simulator also has to consider the migration energies of both constituents. For instance, two rates are needed for $B I_{2} \rightarrow B i+I$, one considering the barrier of $E_{m}(B i)$ and the other $E_{m}(I)$.

Cs can react with MPs and other Cs as long as the formation energy of the final result is included as a parameter. Even in those cases, a probability to reject the reaction

$$
P=\exp \left(\frac{E_{f}^{i}-E_{f}^{f}}{k_{B} T}\right)
$$

is defined to account for the barriers involved in the formation of the new cluster. If $E_{f}^{f}<E_{f}^{i}$ the reaction always happens.

A particular case of Cs are irregular agglomerations of $I$ and $V$ with a non-instantaneous recombination rate. These Cs are normally referenced as $I V$-clusters or Amorphous Pockets, $A P$ s. This mechanism simulates the recombination time needed by IV pairs in some systems, that although small, is not null, to annihilate both defects, Mok et al. (2008); Gomez-Selles et al. (2015). The rates associated with this mechanism can be activated for every cluster, and are computed through

$$
\nu=\nu_{0} \exp \left(-E_{\text {recomb }}(\text { size }) / k_{B} T\right) .
$$




\subsection{Some Examples}

In this section, different examples of simulations upon the kind of results that can be obtained with MMonCa are presented. In Section 2.4.1 the simulator is used to solve the $1 \mathrm{D}$ diffusion equation, while in Section 2.4 .2 experiments upon $I$-type cluster dissolution are reproduced.

\subsubsection{Theoretical particle diffusion}

Let's consider the diffusion equation (Fick's second law) over a particle conduction problem over an isolated line extending in the $x$ coordinate from 0 to $l$, satisfying:

$$
\begin{gathered}
\rho_{t}=D^{2} \rho_{x x} \\
\rho(0, t)=\rho(l, t) \\
\rho_{x}(0, t)=\rho_{x}(l, t) \\
\rho_{x}(x, 0)=\frac{K}{2}\left[1-\cos \left(\frac{2 \pi}{l} x\right)\right]
\end{gathered}
$$

for $0<x<l$ and $t>0$.

The analytical solution $\rho(x, t)$ of such system is:

$$
\rho_{x}(x, t)=\frac{K}{2}\left[1-\cos \left(\frac{2 \pi}{l} x\right) \exp \left(-\frac{4 \pi^{2} D}{l^{2}} t\right)\right]
$$

By interpreting the function $\rho(x, t)$ as the concentration of particles at the position $x$ and time $t$, the boundary condition (2.6) specifies the same concentration at both ends of the line for all time. As in this context, the derivative $\rho_{x}$ represents the particle concentration flux, the boundary condition in (2.7) indicates that the flux of particles exiting at $x=l$ is the same as the flux entering at $x=0$ and vice-versa. In other words, these periodic boundary conditions lead to the assumption that there is a perfect contact and continuous particle flux when the bounds $x=0$ and $x=l$ coincide. In this sense, the problem may be interpreted as particle transport inside a circle with radius $l / 2 \pi$, which is isolated in all the perimeter. 
Fig. 2.7 shows the comparison between the analytical solution 2.9 for $K=10^{20} \mathrm{~cm}^{-3}$, $l=100 \mathrm{~nm}$ and $D=100 \mathrm{~nm}^{2} / \mathrm{s}$ (lines) and KMC simulations (symbols) of the temporal evolution of an initial distribution of particles as the one in (2.8). The KMC simulations have been run up to 10 seconds in a $100 \times 300 \times 300 \mathrm{~nm}^{3}$ simulation cell with a total number of 448820 particles following the initial distribution of 2.8. The diffusivity of each particle was set to $100 \mathrm{~nm}^{2} \mathrm{~s}^{-1}$ through a diffusion rate, at a temperature $T=1000^{\circ} \mathrm{C}$, of:

$$
\nu_{\text {mig }}=5.903 \times 10^{-10} \exp \left(-\frac{0.7}{k_{B} T}\right)
$$

Reactions between particles were not allowed, and periodic boundary conditions were set, meaning that, as in (2.6), particles that jumped further than $x>100 \mathrm{~nm}$, appeared from $x>0 \mathrm{~nm}$.

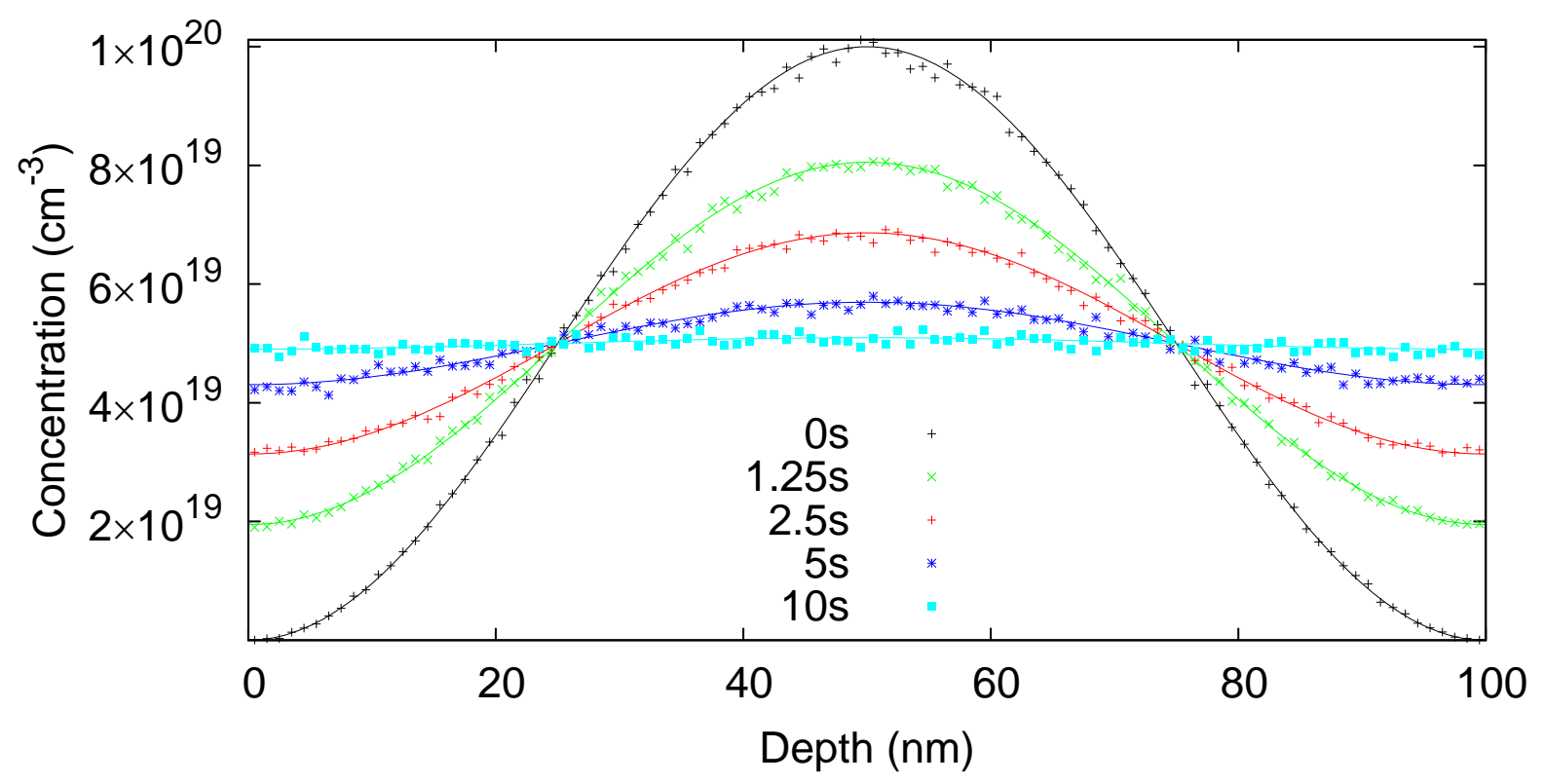

Figure 2.7: Comparison between KMC (symbols) and the theoretical solution (lines) for the time evolution of a spatial concentration of non-interacting particles with periodic boundary conditions. 


\subsubsection{Si Extended Defects dissolution}

In this section, OKMC is applied to reproduce experimental results of well known practical examples of $I$-type cluster dissolution in $\mathrm{Si}$. In fact, as introduced in Chapter 1. the evolution of defects in silicon has been a subject of intense research for the past decades. One particular subject of study has been the characterization of damage by Si implantation. The evolution of such system contains many phases that are nowadays well known, Claverie et al. (2003) and have already been successfully modeled, Martin-Bragado et al. (2008) . The initial implantation produces a high population of $I$ s and $V$ s, where the $V$ diffuses, even at room temperature implantations. During this initial stage $I \mathrm{~s}$ and $V \mathrm{~s}$ do not recombine instantaneously, and tend to form $A P$ s of various sizes. Depending on the particular implantation conditions, the amorphous pocket population might in some cases grow big enough to partially amorphize the sample. In other cases, dynamic annealing of the generated damage, that is, the annihilation of $I V$-pairs during a cascade and the next one, might be enough to avoid amorphization.

Once the implantation has finished, the sample is processed to anneal out the defects. This typically eliminates all the $A P$ s, leaving only small extended defects in the beginning. Such extended defects are composed of the extra Is introduced by the implantation. During the annealing, the small, irregular $I$-type clusters emit their constituent particles. This produces an almost conservative Ostwald ripening where big defects grow at the expense of small ones. At some point, the defects are big enough to be seen through the microscope, getting a characteristic $\{311\}$ shape. Further annealing of these defects produces its dissolution or the formation of very stable dislocation loops.

Parameterizations of formation and migration rates of $I$ s and $V \mathrm{~s}$, Sentaurus (2012); Bracht et al. (1998); Bracht (2000), and formation and dissolution of APs, Mok et al. (2008); Sentaurus (2012) I-type clusters, Martin-Bragado et al. (2008) and voids Sentaurus (2012) are provided in the literature, and have been included in the simulator. With such parameterization, included in every type of simulated object in OKMC, and shown in Table 2.1 two experiments have been reproduced to validate the simulator MMonCa.

Figure 2.8 is a comparison between the experimental, Eaglesham et al. (1994); Stolk et al. (1997) and simulated results of the $\{311\}$ defects dissolution in Si. After a $5 \times$ $10^{13} \mathrm{~cm}^{-2} 40 \mathrm{keV}$ implant of $\mathrm{Si}$ ions into $\mathrm{Si}$, the damage is in the form $A P$ s. Anneals of 


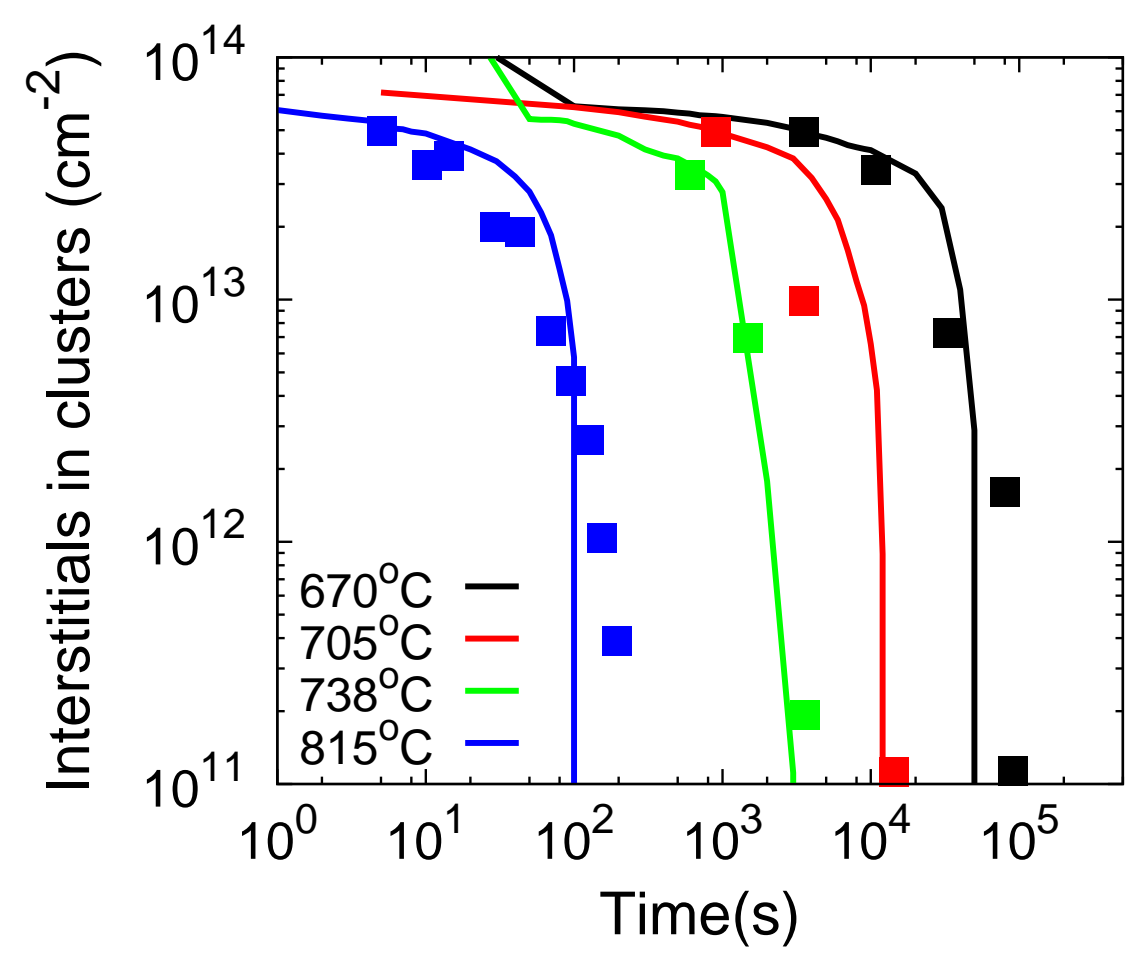

Figure 2.8: Concentration of Si $I$ s in $\{311\}$ defects as a function of time for three different temperatures after a $40 \mathrm{keV}, 2 \times 10^{13} \mathrm{~cm}^{-2}$ Si into Si implant. Symbols: Experimental data taken from Ref.Eaglesham et al. (1994); Stolk et al. (1997), lines: simulation results using MMonCa.

$670,705,738$ and $815^{\circ} \mathrm{C}$ for different times are performed after the implantation, causing full $A P$ recombination. The resting damage is in the form of $\{311\}$ s, which are also dissolved during the annealing time. Satisfactory agreement is found when comparing with the experimental results, as in previous works, Martin-Bragado et al. (2008).

Figure 2.9 represents the comparison of experimental supersaturation (concentration of $I \mathrm{~s}$ in equilibrium versus measured concentration) with the simulated results using MMonCa. The experimental results are taken from Cowern et al. (1999). In the experiment, an implantation of $40 \mathrm{keV}, 2 \times 10^{13} \mathrm{~cm}^{-2}$ Si into Si was followed by annealing at 600,700 and $800^{\circ} \mathrm{C}$ for different times. It is worth reminding the importance of the parameterizations shown in Table 2.1. The mechanisms of migration, formation and dissolution of defects are taken into account in the model, according to the cited references. 


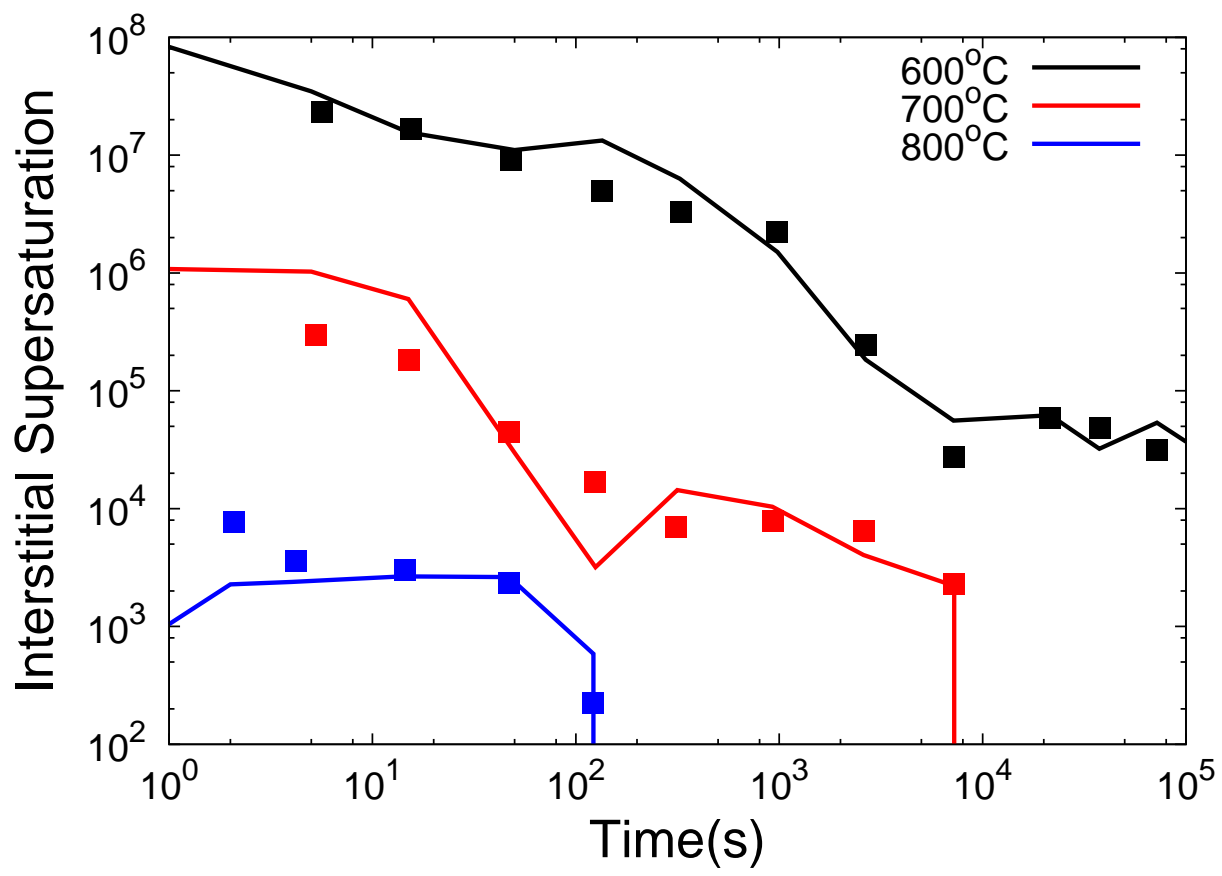

Figure 2.9: Interstitial supersaturation as a function of time after a $40 \mathrm{keV}, 2 \times 10^{13} \mathrm{~cm}^{-2}$ Si into Si implantation at different temperatures 600,700 and $800^{\circ} \mathrm{C}$. Symbols: Experimental data taken from Cowern et al. (1999), lines: simulation results using MMonCa.

Excellent agreement with experimental data, Cowern et al. (1999) is achieved. Both Figs. 2.8 and 2.9 have been produced with the same set of parameters.

Table 2.1: OKMC Silicon model

\begin{tabular}{lcll|}
\hline Object & Migration & Species & Parameters \\
\hline Interface & & $I, V$ creation & Sentaurus $(2012)$ \\
Mobile Particle & Yes & $I$ and $V$ & Bracht et al. (1998); Bracht \\
Cluster & No & $I_{n} V_{n}$ & Mok et al. (2008); Sentaurus \\
Cluster & No & $\{311\} I_{n}$ clusters & Martin-Bragado et al. (2008) \\
Cluster & No & $V_{n}$ voids & Sentaurus (2012) \\
\hline
\end{tabular}




\subsubsection{OKMC modeling of Si amorphization}

Mok et al. (2008) studied amorphization of Si my means of ion implantation using OKMC modeling techniques. The presented model in the cited reference used a threshold value of damage quantity for which the crystalline lattice relaxed into the amorphous phase, i. e. an amorphization threshold. By using this approach, it was demonstrated that OKMC can be used to take into account dynamic annealing effects in terms of $I V$-pairs recombinations being able to consider the impact of different implantation conditions such as the ion-mass, temperature, fluence or flux.

Differently to the models presented in this Chapter and in Chapters 3 and 4 , where those used, improved or developed during this dissertation are presented, the amorphization model discussed in Chapter 5 was written from scratch, of course based on previous works and references, and thus, the technicalities and the dissection of the implementation for this previous model applied for $\mathrm{Si}$ is not done here. Moreover, the specific model of amorphization developed in this work is embedded in Chapter 5, providing the reader a more comprehensive storyline for the specific aspect of OKMC modeling of amorphization in Ge.

\subsection{Conclusions}

After presenting the KMC algorithm, focusing on the OKMC approach and the particular implementation into an actual simulator, published in Martin-Bragado et al. (2013), the simulator has been used to solve and/or reproduce both analytical, theoretical problems and experiments for Si damage mechanisms after ion implantation. These comparisons have been used to validate the presented models and implementation for very well known problems. KMC approaches allow to compute the time evolution of defects at a given rate extracted from experiments or theoretical calculations. It will allow us to use it to reproduce and understand mechanisms of dopant implantation and damage evolution for different conditions in other materials, as Ge. 


\section{Lattice Kinetic Monte Carlo}

In this Chapter, the particular implementation of atoms and possible growth positions lying in the amorphous/crystalline $(\alpha / \mathrm{c})$ interface as objects for the KMC algorithm is introduced as Lattice Kinetic Monte Carlo (LKMC). A direct application of this approach is given for simulating Solid Phase Epitaxial Regrowth (SPER) in Si, along with existing modelization and results for known orientation, defect formation and stress dependence of the SPER process. The bi-modal growth of $\mathrm{Si}(111)$ substrates is explained by an examination of the atomistic structures formed in the process.

\subsection{Introduction}

As it has already been introduced in Chapter 1, amorphization of semiconductor materials is a widely used technique in the microelectronic industry to prevent channeling of dopants and achieve shallow junctions in Si, Ge, and generally Si-based technologies. Doping a crystalline material can yield to amorphous regions produced by damage accu-

mulation. This damaged material is commonly recrystallized by means of SPER, Csepregi et al. (1996), a mechanism of layer-by-layer recrystallization from the amorphous $(\alpha)$ to crystalline (c) phase, achieved by annealing the damaged sample at relatively high temperatures.

SPER is a very complex process in which several mechanisms take place simultaneously, 
such as defect and facet formation, anisotropy and dopant redistributions (See MartinBragado (2012) and references therein).

Simulation of such phenomena can be achieved through different approaches. Molecular Dynamics (MD) techniques are able to offer a physical computation of the fundamental processes at an atomic scale in terms of thermal vibrations and interatomic potentials Bernstein et al. (2000). Nevertheless, MD normally does not fulfill the sizes and timescales required by the industry for actual front-end semiconductor processing, and simulations need to be done at extremely high temperatures for times at the nanosecond scale. Alternatively to MD, Lattice Kinetic Monte Carlo (LKMC) simulation has shown to successfully take into account the important physical mechanisms of crystal growth being also able to meet realistic semiconductor material processing scales, Martin-Bragado and Sklenard (2012).

In this Chapter, a comprehensive LKMC model of atomistic recrystallization processes that includes twin formation and stress dependencies is reviewed. The model is implemented in the LKMC module of the Open Source Kinetic Monte Carlo simulator for damage irradiation evolution and defect diffusion, MMonCa Martin-Bragado et al. (2013), and is described in section 3.2 . Section 3.3 is a compendium of some capabilities of such model and a validation by comparing with experimental results taken from the literature. Conclusions are presented in Section 3.4 .

\subsection{Modeling Solid Phase Epitaxial Regrowth}

Our model simulates the crystalline phase by placing the atoms in a perfect lattice and leaving the amorphous phase as a source of atoms that can attach to a neighboring crystalline phase. Following this definition, there are atoms in the interface with free bonds that need to be filled attaching to the crystalline phase in order to complete the perfect lattice in a specific regrowth direction. These sites are filled with atoms following certain rates.

Such rates are given by an Arrhenius law, defined by the SPER process, and different for each material. For a given temperature $\mathrm{T}$ the rate is thus computed as 


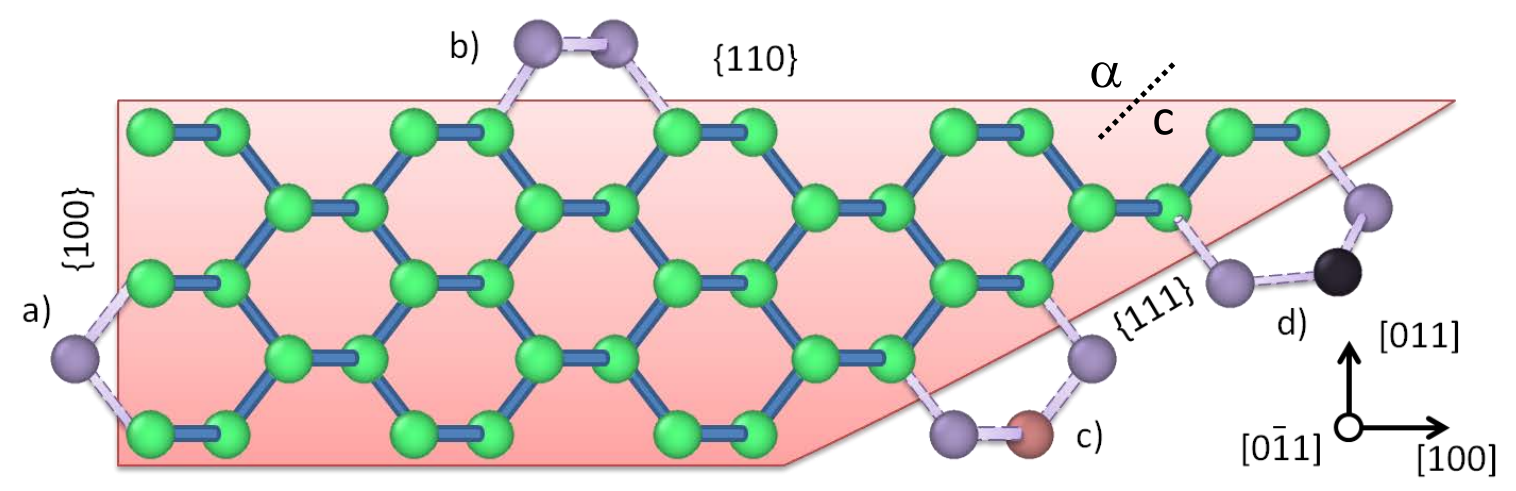

Figure 3.1: Sketch of the lattice atoms (in green) arranged in the crystalline phase (red area), and the three possible different growth directions perpendicular to the family of planes: a) $\{100\}$, b) $\{110\}$, c) and d) $\{111\}$, of the $\alpha /$ c transition through the interface. Non-deffective and twin growing sites through the direction perpendicular to the $\{111\}$ family of planes, c) and d) respectively, are also represented.

$$
\nu_{0}=K(\text { site }) \exp \left(-\frac{E_{a}}{k_{B} T}\right)
$$

where $K$ (site) are the prefactors, that depend on the local microscopic growth direction and $E_{a}$ is a constant activation energy. Thus, basically, these rates can be understood as the number of atoms per unit time that will be attached from the $\alpha$ - to the c-phase depending on the number of available bonds at the interface able to continue forming a Si lattice from the crystalline seed with the bonds undistorted. Such dependence is given by the prefactor, $K($ site $)$. The available possible positions are calculated and stored in a table where each of them will have a different rate to recrystallize.

Figure 3.1 is a sketch of the lattice atoms (in green) arranged in the crystalline phase (red area), and the three possible different growth directions perpendicular to the family of planes $\{100\},\{110\}$ and $\{111\}$, of the $\alpha /$ c transition through the interface. The rates represented in Eq. 3.1 refer to the events of recrystallizing atoms from the amorphous phase (i.e. out of the red area). It is easy to appreciate that, following Drosd and Washburn (1982): a) $\{100\}$ sites need only one atom to incorporate to the c-phase in order to grow; b) the $\{110\}$ family needs two atoms per event; c) and d) $\{111\}$ sites need three new 
crystalline atoms to fulfill the next growing step.

It has been reported that highly coordinated sites, that is, with more than eight first and second crystalline neighbors, present a faster growth velocity than configurations with low coordination (less than eight first and second crystalline neighbors) $\{100\}_{l}$, Williams and Elliman (1983). In our model, this is taken into account by unfolding the $\{100\}$ rates into $\{100\}_{h}$ and $\{100\}_{l}$ for high and low coordinated configurations, respectively, discriminating between more or less than eight first and second neighbors.

Twin defect formation has been reported to be the main source of defects during SPER, Martin-Bragado (2012); Martin-Bragado and Sklenard (2012). In our model, this is taken into account by detecting all possible recrystallization configurations. In particular, when a $\{111\}$ site is to be filled, (see Fig. 3.1) the lattice is not able to distinguish between: c) non-defective and d) twin positions, being both equally valid growing alternatives and thus serving as a seed for twin growing.

Finally, SPER rate dependence on a stress state, $\boldsymbol{\sigma}$, is introduced in this model by following the theoretical investigations carried out by Aziz et al. (1991). In such approach, an activation strain tensor, $\boldsymbol{V}^{*}$, is introduced in the stress-free rate formulation of Eq. 3.1. A simplified model than the one with a four order tensor reported in Sklenard et al. (2013), and only using a second order tensor, has proven to be enough to model these SPER stress effects, Sklenard et al. (2014). Thus, Eq. 3.1 is expanded as:

$$
\nu\left(\sigma_{i j}\right)=\nu_{0} \exp \left(-\frac{V_{i j}^{*} \sigma_{i j}}{k_{B} T}\right),
$$

being $\mathrm{V}_{i j}^{*}$ unfolded upon the normalized regrowth direction $\boldsymbol{d}$ :

$$
V_{i j}^{*}=V_{\|}^{*} d_{i} d_{j}+V_{\perp}^{*}\left(\delta_{i j}-d_{i} d_{j}\right)
$$

where $V_{\|}^{*}$ and $V_{\perp}^{*}$ are the dimension change in the parallel and perpendicular directions respectively. These strain activation tensors depend on the number of crystalline first nearest neighbors (c1NN). Further discussion on this model can be found in Refs. Sklenard et al. (2013, 2014). Of course, if the stress applied to the substrate is null, Eq. 3.2 becomes again to the form of Eq. 3.1.

As a summary of this section, it can now be concluded that the objects that are to be simulated by LKMC in this approach are the available lattice sites at the very $\alpha / c$ interface. 
These sites are calculated based on the first layers of the c-phase next the interface itself. The rates of production of new crystalline atoms (from the $\alpha$-phase) depend on the local configuration of each site (See Eq. 3.1 and Fig. 3.1). $\{100\}$ rates will also depend on the number of first and second crystalline neighbors. New $\{111\}$ growth configurations are not able to distinguish between non-defective and twin-defective configurations, which, as it will be seen in Section 3.3 will have a direct impact on the SPER rates for nearly every substrate orientation. These rates are also changed by an exponential term (Eq. 3.2) if a stress field is applied.

\subsection{SPER in Si}

In this Section, the presented models are used as an example to validate the model before using it into reproducing Ge experiments in Chapter 6. In Table 3.1 the calibrated values for the prefactors depending on the local atomistic configuration of the regrowing Si lattice site are presented, after Martin-Bragado (2013). The same activation energy in Eq. 3.1 of $2.7 \mathrm{eV}$ is used. Table 3.2 comprises the particular calibration for the activation strain tensors of Eq. 3.2 in Si, after Sklenard et al. (2014).

\subsubsection{Different orientations}

Csepregi et al. (1978) measured experimentally the SPER velocity dependence on the substrate angle at $550{ }^{\circ} \mathrm{C}$ for different times. The results showed that growing a $\mathrm{Si}(100)$ substrate is about one order of magnitude faster than the same experiment performed for $\mathrm{Si}(111)$, with the $\mathrm{Si}(110)$ substrate having a velocity in between these two. The prefactors, $K($ site), for each local configuration shown in Table 3.1 follow the same trend, along with the observations of Williams and Elliman (1983) for high and low coordinated $\{100\}$ sites.

Figure 3.2 is a comparison between measured, Csepregi et al. (1978), and simulated regrowth velocities for $\mathrm{Si}$ at $550{ }^{\circ} \mathrm{C}$. Correspondences between angles and crystallographic directions are also shown. The angles are referred with respect to the $\mathrm{Si}(100)$ substrate. It is remarkable that twin defect formation along $\{111\}$ facets is needed to successfully reproduce the experimental measurements. Recrystallization rates used in LKMC with 
an activation energy of $2.7 \mathrm{eV}$ and the prefactor calibration shown in Table 3.1 are able to achieve an excellent match between experimental and simulation results. This is only performed by being able to take into consideration the twin defect formation, MartinBragado (2012), as will be discussed in Section 3.3.2.

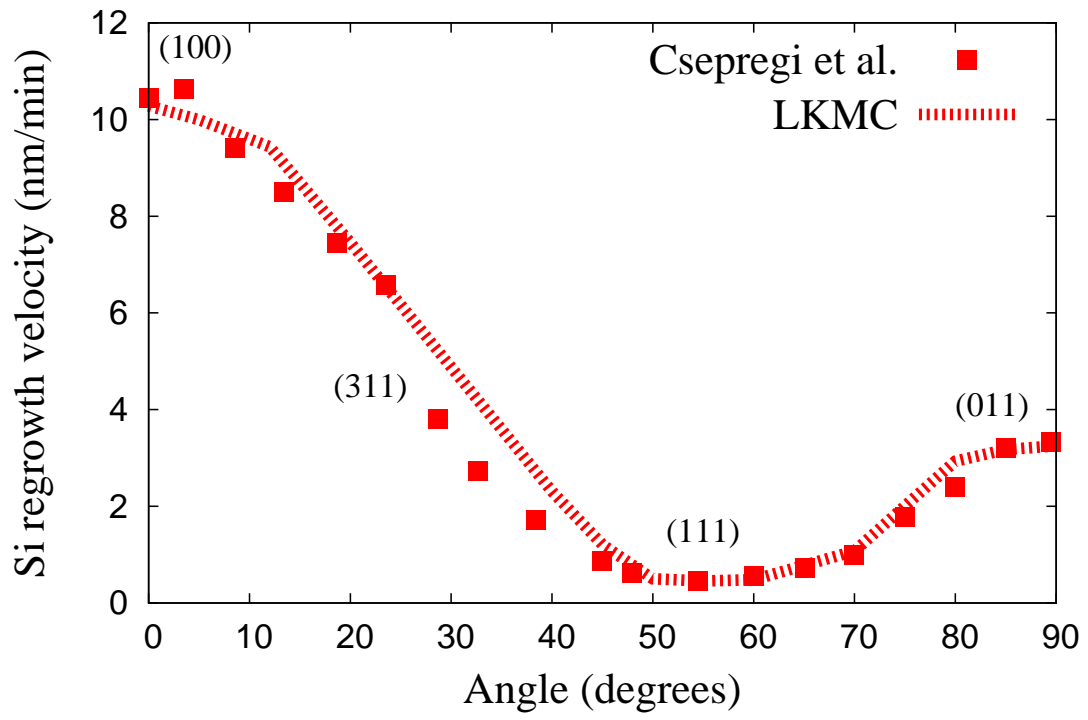

Figure 3.2: Comparison between experimental, Csepregi et al. (1978), and simulated results of $550{ }^{\circ} \mathrm{C}$ Si SPER velocities depending on the substrate regrowth orientation. The angles are referred with respect to the (100) regrowth direction.

Table 3.1: Prefactors and activation energies for each local recrystallization configuration for Si. After Martin-Bragado (2013).

\begin{tabular}{ll}
\hline \hline Site & $K($ atoms $/ \mathrm{s})$ \\
\hline $100_{h}$ & $7.42 \times 10^{17}$ \\
$100_{l}$ & $3.09 \times 10^{16}$ \\
110 & $2.08 \times 10^{15}$ \\
111 & $1.62 \times 10^{12}$ \\
\hline \hline
\end{tabular}




\subsubsection{Twin defects}

It has been reported that regrowth of $\mathrm{Si}(111)$ substrates leads to two different SPER velocity regimes, Csepregi et al. (1976). These results have been reproduced by LKMC simulations through the inclusion of a twin defect formation mechanism, Martin-Bragado and Sklenard (2012), as explained in Section 3.2 .

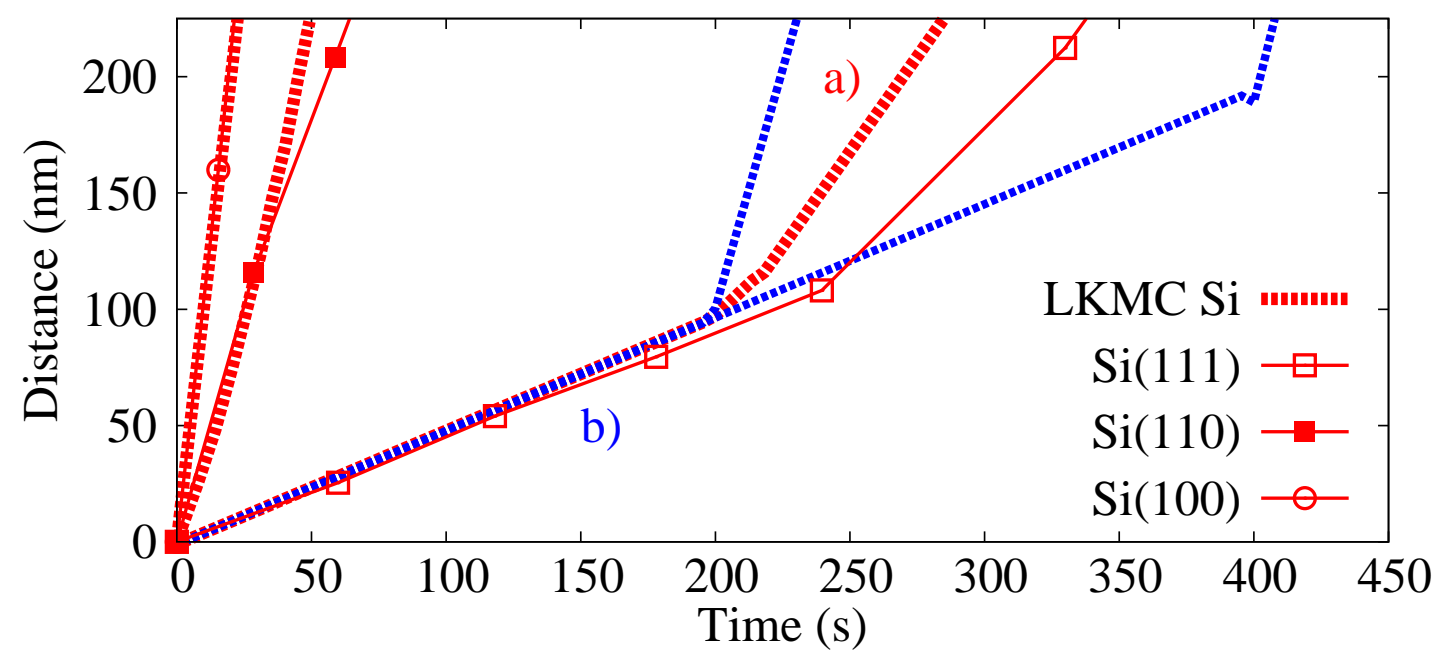

Figure 3.3: Simulated (dashed lines), Martin-Bragado and Sklenard (2012), and experimental (thin solid lines and symbols), Csepregi et al. (1976), results of regrown distance with time for $\mathrm{Si}(100), \operatorname{Si}(110)$ and $\operatorname{Si}(111)$ substrates. Two different velocity regimes are found for $\mathrm{Si}(111)$, where a) a mean between simulations performed for different random seeds is presented, and b) Examples of the results obtained with those different seeds are shown in blue dashed lines.

Figure 3.3 is a comparison between simulated and measured, Csepregi et al. (1976), amount regrown over time for $\mathrm{Si}(111), \mathrm{Si}(110)$ and $\mathrm{Si}(100)$ substrates at $550{ }^{\circ} \mathrm{C}$. A very good agreement between experiments and simulations was found. By taking into account twin defect formation, the appearance of these two regimes can be explained as a result of defective seeds that provoke a suddenly faster SPER velocity. To make this point clear, an atomistic investigation on the mechanism that take place in Si SPER has been carried out, clarifying the nature of the seed for the bi-modal regrowth.

Figure 3.4 is a representation of the growing process of one of these defective seeds taken 


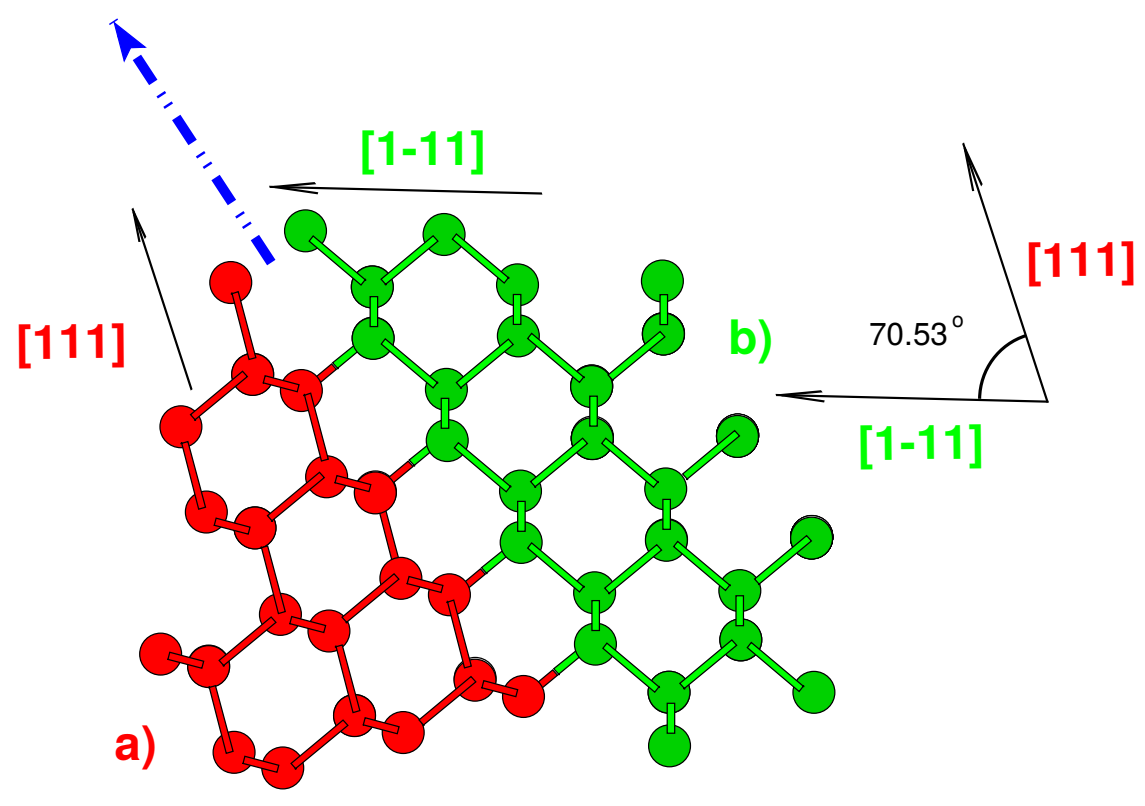

Figure 3.4: Atomistic representation of the growing process caused by the engagement of a [111] facet, a) (in red) and b) (in green) [1-11]. For the specific configuration shown here, both facets can join (red-green bonds) and grow faster by a mixed $\{110\}$ microscopic event (blue dashed line), which serves as an unexpected seed for the growth of the crystal.

from one of our LKMC simulations. When a facet a) which is growing in a, for example, [111] direction, encounters a specific twin of a different facet, b), which is recrystallizing at a specific direction, [1-11] (as shown in Figure 3.4 forming an angle of $70.53^{\circ}$, there is a statistical probability for both facets to "engage" in a way that further atoms are able to grow in a preferential and faster direction (blue dashed line) since microscopically the sites are to grow by filling the new $\{110\}$ sites. Thus, in Figure 3.3 a mean of simulations performed with different random seeds is presented for the $\mathrm{Si}(111)$ substrate. Although this process dramatically changes the defects generated in the growing direction, given its very low probability (since small deformations prevent this structure to be formed), it is not likely to occur in the microelectronic processing scales. 


\subsubsection{Hydrostatic pressure}

A study on Si SPER upon the appliance of hydrostatic pressure carried out by Lu et al. (1991) showed that there is an increment in the velocity with increasing hydrostatic pressure. Sklenard et al. (2014) used the model described in Section 3.2 to atomistically describe the Si SPER process under different stress conditions using LKMC simulations. Such different experiments were taken from Rudawski et al. (2008) for in-plane uniaxial stress, Barvosa-Carter (1997) for normal uniaxial compressive stress and the mentioned Lu et al. (1991) measurements for SPER under hydrostatic pressure. The resulting calibration achieved for the values of the components of the activation strain tensors depending on the number of crystalline first neighbors $(\mathrm{c} 1 \mathrm{NN})$ in Si is shown in Table 3.2 .

Such calibration was based in understanding the different limiting factors that, under certain stress conditions, produced in the atomistic growth of the recrystallizing layers as a function of the number of crystalline neighbors of the $\{100\}$ sites. Figure 3.5 is a comparison between experimental results taken from Lu et al. (1991) for a $530{ }^{\circ} \mathrm{C}$ anneal, and LKMC simulations providing further validation of the model.

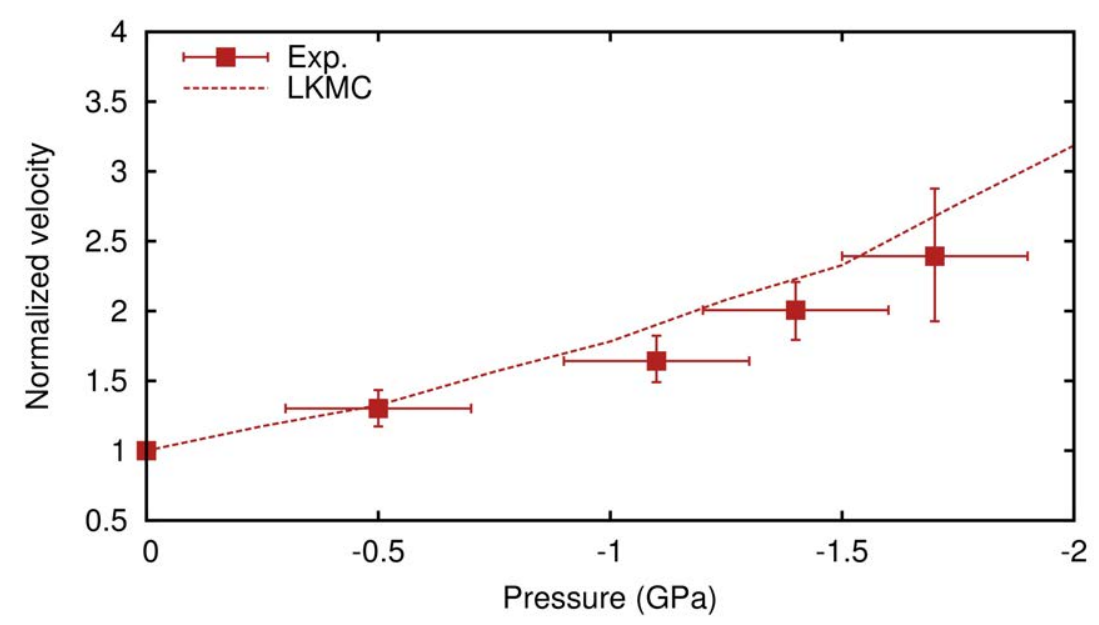

Figure 3.5: Normalized regrowth velocity of the $\mathrm{Si}(100)$ interface as a function of hydrostatic pressure at $530{ }^{\circ} \mathrm{C}$. LKMC simulations results (dashed line) are compared with experimental measurements of Lu et al. (1991). After Sklenard et al. (2014). 
Table 3.2: Values of the components of the activation strain tensors depending on the number of crystalline first neighbors (c1NN) in Si. $\Omega_{S i}$ is the molar volume of Si. After Sklenard et al. (2014).

\begin{tabular}{lcc}
\hline \hline $\mathrm{c} 1 \mathrm{NN}$ & $V_{\|}\left(\Omega_{S i}\right)$ & $V_{\perp}\left(\Omega_{S i}\right)$ \\
\hline 2 & -0.33 & 0 \\
3 & -26 & 13 \\
\hline \hline
\end{tabular}

\subsection{Conclusions}

In this Chapter, modeling of SPER through a LKMC algorithm has been explained. In this case, the objects to be simulated have been the lattice sites at the $\alpha / \mathrm{c}$ interface. These sites are calculated and stored in a table containing all their associated rates. The rates of site recrystallization depend on the local configuration of each site. This dependence is modeled by using the same activation energy but different prefactors for $\{100\},\{110\}$ and $\{111\}$ sites. Twin defects appear as a natural consequence of the $\{111\}$ recrystallization process. This simple modelization of the SPER process is able to explain and reproduce the orientation dependence of the SPER process in Si as well as the bi-modal growth reported for $\mathrm{Si}(111)$ substrates. A dependence of these rates on the activation stress tensors for $\{100\}_{2}$ and $\{100\}_{3}$ sites is able to explain the SPER process under different stress conditions. This model, will allow us to understand the SPER process in Ge in Chapter 6 . 
Chapter 4

\section{BINARY COLLISION APPROXIMATION}

In this Chapter, a brief introduction to the Binary Collision Approximation (BCA) Monte Carlo technique is given. The fundamental equations and the assumptions that this method encompass are provided along with the differences between the models used for modeling ion implantation into an amorphous or crystalline target. In the first case, the number of collisions is given by the density of the material while for crystalline targets, an ideal lattice of the target is simulated in the ion path to calculate the collisions. This approximation will naturally lead to be able to simulate channeling effects in the ion implantation process, that strongly depend on the substrate orientation and the incident angle of the incoming ions.

\subsection{Introduction}

In order to simulate the trajectories, the generated damage and final doping profiles generated by implanting ions into semiconductor materials, there are two group of techniques that are normally used.

Molecular Dynamics (MD), once again, is able to provide accurate quantities and distributions of both damage and doping profiles, Caturla et al. (1996). Nevertheless, the simulation costs in terms of time and computational resources are too high for MD to be able to serve as a fast and functional tool for simulating very different conditions of ion 
implantation. Thus, BCA is generally used in semiconductor materials for extracting this type of information and has helped for many years as a tool for both making predictive models, Mok et al. (2008) and for experimentalists to be able to extract conclusions on the mechanisms underlying very complex experiments, Napolitani et al. (2014).

\subsection{How BCA works?}

BCA is a Monte Carlo method in which the individual trajectories of ions entering the target are simulated. The Monte Carlo approach relies on the randomness applied to the initial conditions of position and velocity. It is called Binary Collision because each time it tracks the interactions between only two particles: the moving ion (or secondary projectiles) and the nearest atom in the target. By considering an incoming ion with an initial kinetic energy, part of this energy will be transfered through elastic nuclear collisions with the target atoms and the rest will be inelastically lost through interactions with the electrons in the ion path, Lindhard and Scharff (1961); Lindhard et al. (1963):

$$
\left[\frac{d E}{d x}\right]_{\text {total }}=\left[\frac{d E}{d x}\right]_{\text {nuclear }}+\left[\frac{d E}{d x}\right]_{\text {electronic }}
$$

When the energy of the ion is considered low enough, the projectile is stopped. This way, final impurity profiles resulting from ion implantation are calculated extracting the dopant positions after simulating the desired fluence.

Regarding the generated damage, if the transfered energy to the target atom is higher than a threshold energy, this atom will be displaced from its position. These considerations allow BCA to track quantity and positions of the damage in the form of $I$ s and $V \mathrm{~s}$ (see Figure 1.2(b).

In this thesis, dopant and damage profiles resulting from ion implantations are calculated using the Sentaurus Process BCA module, Sentaurus (2012). Despite there are several approaches taken in the literature and in different simulators, Ziegler et al. (2008), MARLOWE, Robinson and Torrens (1974) or IIS, Hernandez-Mangas (2000) to name a 
few, those will no be reviewed, since they have not been used in this work. In the used simulator, the classical notation used in Eq. 4.1 is transformed to:

$$
E_{i}=E_{i-1}-\Delta E_{n}-\Delta E_{e}
$$

meaning that the total energy loss of the ion after the $i$-th collision is given by the sum of both nuclear, $\Delta E_{n}$, and electronic, $\Delta E_{e}$, energy losses.

The first term of Eq. 4.2 is calculated according to the classical binary scattering theory from classical mechanics, Goldstein (1957).

\subsubsection{Nuclear collisions}

Considering a particle of mass $M_{1}$ traveling with a kinetic energy $E_{0}$ approaching to a stationary particle of mass $M_{2}$, the impact parameter $b$ is defined as the distance of closest approach if the particle is not deflected, giving the idea of of how close the collision is. After the collision, the first particle is deviated an angle $\theta$.

$$
\Delta E_{n}=E_{0} \frac{4 M_{1} M_{2}}{\left(M_{1}+M_{2}\right)^{2}} \cos ^{2}(b I)
$$

and $I$ is the integral

$$
I=\int_{0}^{s_{\max }} \frac{d s}{\sqrt{1-\frac{V(s)}{E_{r}}-b^{2} s^{2}}}
$$

where $s=1 / r$ is the inverse separation between the two particles. $V(s)$ is the (repulsive) potential between those two particles and

$$
E_{r}=\frac{E_{0}}{1+M_{1} / M_{2}}
$$

is the reduced energy in the center of coordinates. The upper limit of the integral, $s_{\max }$, is the inverse distance of closest approach of the two particles, given by solving:

$$
1-\frac{V\left(s_{\max }\right)}{E_{r}}-b^{2} s_{\max }^{2}=0
$$

The scattering angle previously defined, $\theta$, by which particle 1 is deflected is given by: 


$$
\cos \theta=\frac{1-\left[\frac{0.5}{E_{0}}\right]\left[1+\frac{M_{2}}{M_{1}}\right] \Delta E_{n}}{\sqrt{1-\frac{\Delta E_{n}}{E_{0}}}},
$$

where $\theta \rightarrow 0$ if $\frac{\Delta E_{n}}{E_{0}} \ll 1$.

The potential, $V(r)$, used is the Coulomb potential between two particles, with atomic numbers $Z_{1}$ and $Z_{2}$, multiplied by a screening factor. This leads to the so called Universal Potential, Ziegler et al. (2008) defined as:

$$
\begin{array}{r}
V(r)=\frac{Z_{1} Z_{2} e^{2}}{4 \pi \epsilon_{0} r}\left[0.18175 e^{-3.19998 r / a_{u}}+0.50986 e^{-0.94229 r / a_{u}}\right. \\
\left.+0.28022 e^{-0.4029 r / a_{u}}+0.028171 e^{-0.20162 r / a_{u}}\right] .
\end{array}
$$

where $a_{u}$ is the universal screening distance:

$$
a_{u}=\frac{0.8854 a_{0}}{Z_{1}^{0.23}+Z_{2}^{0.23}} \mu \mathrm{m}
$$

Depending on if the target to be simulated is amorphous or crystalline, two different models are used to calculate the ion trajectories.

\section{Amorphous target}

For amorphous targets, the scattering events are assumed to occur in the material after traveling a distance:

$$
L=1 /\left(N_{\text {dens }}\right)^{1 / 3}
$$

where $N_{\text {dens }}$ is the atomic density of the target. The target atom is approached with the impact parameter, $b$, by the incident ion. The probability of finding an atom between $b$ and $b+\delta b$ is given by the distribution $w(b) \delta b=2 \pi N_{\text {dens }}^{2 / 3} b \delta b$. By choosing a random number, $R_{\text {rand }}$, uniformly distributed between 0 and 1 , such distribution gives:

$$
b=\sqrt{\frac{R_{\text {rand }}}{\pi N_{\text {dens }}^{2 / 3}}},
$$

allowing to calculate the energy lost by atomic collisions given by Eq. 4.3 . 


\section{Crystalline target}

The calculation is performed in the same way as for amorphous targets but, instead of approximating a distance between collisions by Eq. 4.10 to find a collision partner through the atomic density of the target material and a random number, such partner is found by calculating the distances between the projectile and an idealized local lattice with a given orientation. Small temperature-dependent effects of lattice vibrations are also considered by slight random variations in the atom positions at equilibrium. This approach is based on the BCA simulator MARLOWE, Robinson and Torrens (1974) with a variation in the turning point of the projectile that improves results for ultralow energy implants, which does not actually impact the conditions studied in this work, Sentaurus (2012). With this approach, ion channeling results as a natural consequence of the target lattice orientation with respect to the incoming ion angle. This way, preferential penetration of implanted ions along crystal axes or planes is achieved. In this case, the effect of tilting or rotating the target can be simulated. For implantations in which channeling occurs, the mechanism of energy loss by electronic interactions becomes more relevant.

\subsubsection{Electronic Stopping}

In this case, no distinction between amorphous or crystalline targets is made, and thus, the same model is used to its calculation. The second term for energy loss in Eq. 4.2 is the weighted sum of both nonlocal, $\Delta E_{e}^{n l}$, and local, $\Delta E_{e}^{l}$, stopping power contributions:

$$
\Delta E_{e}=w_{1} \Delta E_{e}^{n l}+w_{2} \Delta E_{e}^{l}
$$

where $w_{1}+w_{2}=1$.

The nonlocal contribution acts as the frictive force on moving ions inside the target, which is independent of the impact parameter and proportional to the traveled length, $L$, and the ion velocity:

$$
\Delta E_{e}^{n l}=L N_{\text {dens }} S_{e}
$$


where $S_{e}$, for a given kinetic energy $E_{m}$ follows, Lindhard and Scharff (1961):

$$
S_{e}=\frac{1.212 Z_{1}^{7 / 6} Z_{2}}{\left[Z_{1}^{2 / 3}+Z_{2}^{2 / 3}\right]^{3 / 2} M_{1}^{1 / 2}}
$$

The last term is the local electronic loss, which is a consequence of the electron exchange between the moving ion and the local target atom, after Oen and Robinson (1976):

$$
\Delta E_{e}^{l}=\frac{S_{e}}{2 \pi a^{2}} \exp \left(-\frac{b}{a}\right)
$$

with $a=\frac{1.45 a_{u}}{0.3 Z_{1}^{2 / 5}}$.

\subsection{Conclusions}

A brief introduction to the BCA method has been given, explaining the models that are used for calculating the damage and doping profiles. As will be seen in Chapter 5, this technique will serve us to simulate the damage caused by every ion. Then, this damage will be introduced in the KMC simulator in order to model the time evolution of these defects in terms of migration, formation of disordered regions, and even amorphization of the Ge sample.

For a more extended information on the BCA method and the methods normally used, the reader is suggested to follow the references: Lindhard and Scharff (1961); Lindhard et al. (1963); Sentaurus (2012); Ziegler et al. (2008); Robinson and Torrens (1974); Oen and Robinson (1976). 


\section{Part II: Results}

"It worked."

— J. Robert Oppenheimer 
CHAPTER 5

\section{Amorphization IN GE BY MEANS OF ION IMPLANTATION}

"Qu'on me donne six lignes écrites de la main du plus honnête homme, j'y trouverai de quoi le faire pendre."

— Armand Jean du Plessis de Richelieu

In this Chapter, damage accumulation and amorphization mechanisms by means of ion implantation in Ge are studied using Kinetic Monte Carlo and Binary Collision Approximation techniques, following the ideas of Mok et al. (2008). Such mechanisms are investigated through different stages of damage accumulation taking place in the implantation process: from point defect generation and cluster formation up to full amorphization of Ge layers. A damage concentration amorphization threshold for Ge of $\sim 1.3 \times 10^{22} \mathrm{~cm}^{-3}$ which is independent on the implantation conditions is proposed. Recombination energy barriers depending on amorphous pocket sizes are provided. This leads to an explanation of the reported distinct behavior of the damage generated by different ions. It has also been observed that the dissolution of clusters plays an important role for relatively high temperatures and fluences. The model, once calibrated, is able to explain different damage generation regimes, amount of generated damage and extension of amorphous layers in Ge for different ions and implantation conditions. The model and results presented 
in this Chapter have been improved and extended from the original work published in Gomez-Selles et al. (2015).

\subsection{Introduction}

A serious effort has been invested in the last years in order to understand the mechanisms of Ge damage accumulation and amorphization caused by ion implantation, Satta et al. (2005); Posselt et al. (2006); Decoster and Vantomme (2009); Koffel et al. (2009); Impellizzeri et al. (2009a); Claverie et al. (2010); Impellizzeri et al. (2011). Specially, Bdoping in Ge is of particular interest due to its low interstitial $(I)$ mediated diffusion, Mirabella et al. (2013). In this context of Ge implementation in ultimate microelectronic devices, wafers are treated with ion-beam techniques in which damage accumulation and amorphization play a significant role, Chao et al. (2005). Thus, it becomes important to understand amorphization processes in order to predict and optimize the position and extension of amorphous layers in all relevant implantation conditions.

Within this framework, the Critical Damage Energy Density (CDED) model, Claverie et al. (1988) was able to accurately reproduce amorphous/crystalline $(\alpha / c)$ interface locations for relatively heavy ions and wide fluence ranges, Koffel et al. (2009). Nevertheless, predictions for low mass ions, such as B, could not be provided. Despite other models also reported consider the competition between damage buildup and its dynamic recovery, Posselt et al. (2006) a deeper understanding of the energetics of recombination and migration barriers was also suggested to be needed.

In this Chapter, we introduce a quantitative atomistic model of damage accumulation and amorphization by means of ion implantation in Ge. Explanations for the differences between the damage caused by ions of different masses are presented and validated by reproducing experimental observations of B, F, Ga and Ge implants. This is achieved through an intensive study of the energetics of recombination of "amorphous" pockets $(A P)$ coupled with a realistic calculation of the damage distribution within the volume of implantation. Damage accumulation mechanisms towards amorphization have been examined. Starting with simulations performed at cryogenic conditions, the pure collisional stages are separated from dynamic annealing effects. Later, simulations of ion implantation 
at higher temperatures where both effects coexist, provide clarifications on different steps of Ge amorphization. Validity of the model is shown by simulating own experiments and others reported in the literature, Satta et al. (2005); Koffel et al. (2009); Impellizzeri et al. (2009a); Posselt et al. (2006); Boninelli et al. (2012b); Claverie et al. (2010).

\subsection{Model}

By coupling a Binary Collision Approximation (BCA) code with a physically based Kinetic Monte Carlo (KMC) method, a complex variety of mechanisms induced by ion implantation can be simulated, Martin-Bragado et al. (2004); Duffy et al. (2013); Mok et al. (2008). Among BCA codes, SRIM, Ziegler et al. (2008) is widely used although it does not consider the crystallinity of the target material. This might lead to inaccurate damage or doping profile predictions, and the models used need to be chosen attending to specific experiments, Stoller et al. (2013). Particularly, light ions are easily affected by channeling effects, Wittmann and Selberherr (2007); Guo et al. (2002). To take this into account, the Sentaurus Process BCA module, Sentaurus (2012) was used to generate the initial damage ( $I$ s and vacancies, $V$ s) distribution. The threshold displacement energy per atom, $E_{t}$, used in this model is $30 \mathrm{eV} /$ atom, Mitchell (1957); Wittmann and Selberherr (2007).

It is widely accepted that damage cascade generation occurs at a different time scale than the dynamic events taking place during the implantation process, Keating (1966). As a consequence, damage due to a single ion can be calculated, and then, its dynamic evolution computed, Mazzone and Servidori (1988). In this model, damage cascades taken from BCA calculations are introduced at the desired rate into the Object-KMC simulator for damage irradiation evolution and defect diffusion, MMonCa, Martin-Bragado et al. (2013) in order to simulate dynamic annealing effects. The damage cascades produced by every single ion are calculated, one by one, in two different sets of simulations: assuming a pure crystalline material ( $c$-cascades) or a pure amorphous one ( $\alpha$-cascades) for each ion, separately (models described in Chapter 4). In particular, Table 5.2 on page 78 shows the cascades used for every condition used in this Chapter. As it will be discussed in Section 5.3.3, the damage quantity and distribution calculated in both sets of simulations 
are different, and it will lead to study the conditions for which $c$ - or $\alpha$-cascades need to be used depending on the ion mass and, for extreme conditions, depending also on the temperature or flux of implantation.

Three different damage generation regimes have been reported for ion implantation in both Ge and Si materials (see Refs. Decoster and Vantomme (2009) and Pelaz et al. (2004) respectively and references therein), but these regimes have not been as studied in Ge as in Si. We model these implantation regimes as follows. Damage is produced in the simulated target, and its production grows slowly until it locally overcomes a certain threshold of damage concentration. At this point, areas of $c$-Ge relax towards the $\alpha$-Ge phase and, as in Si, Pelaz et al. (2004) the mixture of these different phases result in a faster damage growth regime. These areas nucleate until a full layer is amorphized and, as the lattice has relaxed, no more damage can be generated. In this new regime, the induced damage only contributes to the widening of the layer.

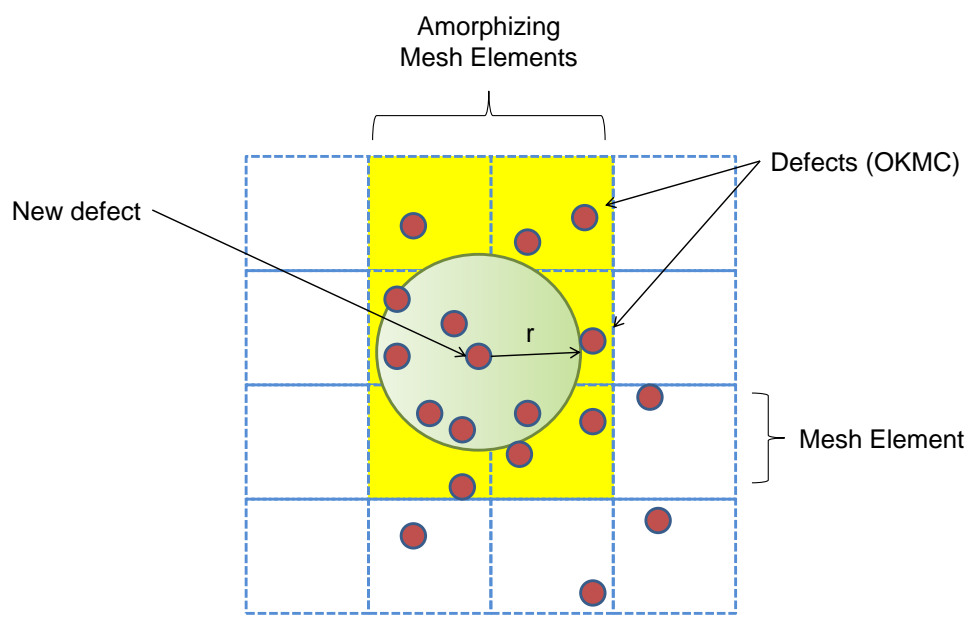

Figure 5.1: 2D-sketch of the Amorphization process. Step 1. Every time an $I$ or $V$ is introduced in the material, the concentration of defects inside the $M E$ s covered by a sphere (in green) of $1 \mathrm{~nm}$ radius, $\mathrm{r}$, is checked in the volume of those $M E$ s susceptible to amorphize (in yellow).

Figures 5.1 and 5.2 are a 2D-sketch of the amorphization model implemented in MMonCa. 
As described in Chapter 2, space is divided into small cubic boxes, called Mesh Elements, $(M E)$. Materials are defined for each $M E$, so the volume of the material that is changed is determined by the units of volume that every $M E$ occupies. As amorphization is a process in which the lattice relaxes towards a new phase, a single $M E$ is not representative of the whole process. Thus, every time an $I$ or $V$ is introduced in the material (see Figure 5.1), the concentration of defects inside the $M E \mathrm{~s}$ (in yellow) covered by a sphere (in green) of $1 \mathrm{~nm}$ radius, $\mathrm{r}$, is checked in the volume of those $M E$ s susceptible to amorphize. If the concentration of defects within that volume overcomes the amorphization threshold, all those $M E$ s are amorphized, (see Figure 5.2) and the atoms forming the $\alpha / \mathrm{c}$ interfaces are placed by the LKMC module (in green). Those defects which are not defined for the amorphous phase are automatically erased from the simulation. Nevertheless, when extracting information from those $M E \mathrm{~s}$ which are amorphous, it is assumed that the whole lattice has lost its crystallinity, and for instance, concentration profiles for both $I$ s and $V$ s will return the material atomic concentration.

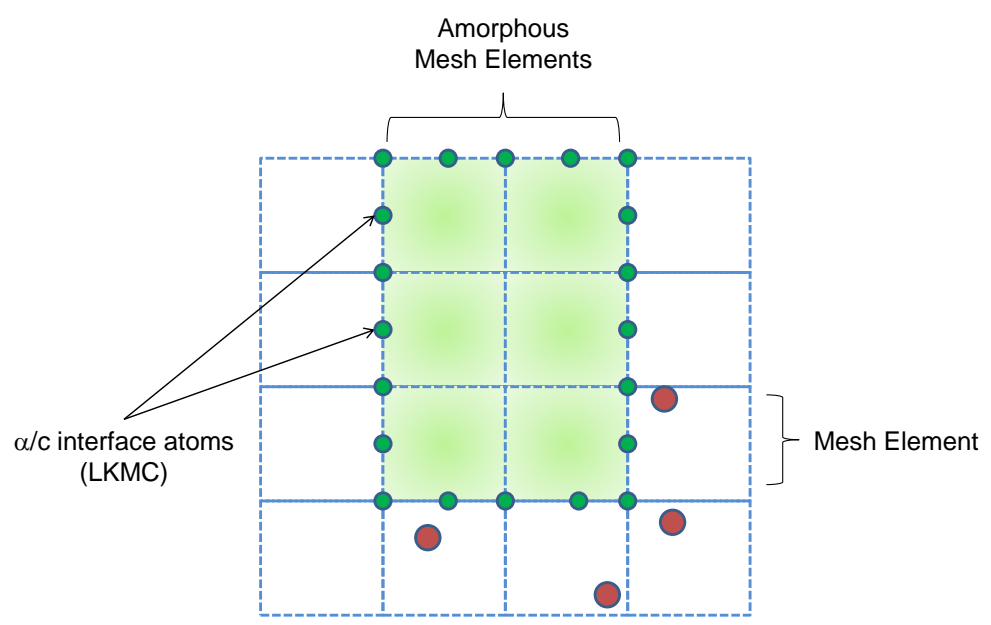

Figure 5.2: 2D-sketch of the Amorphization process. Step 2. Atoms forming the $\alpha / \mathrm{c}$ interfaces are placed by the LKMC module (in green). Those defects which are not defined for the amorphous phase are automatically erased from the simulation.

The result of applying this model into a realistic simulation is shown in Figure 5.3. First, 


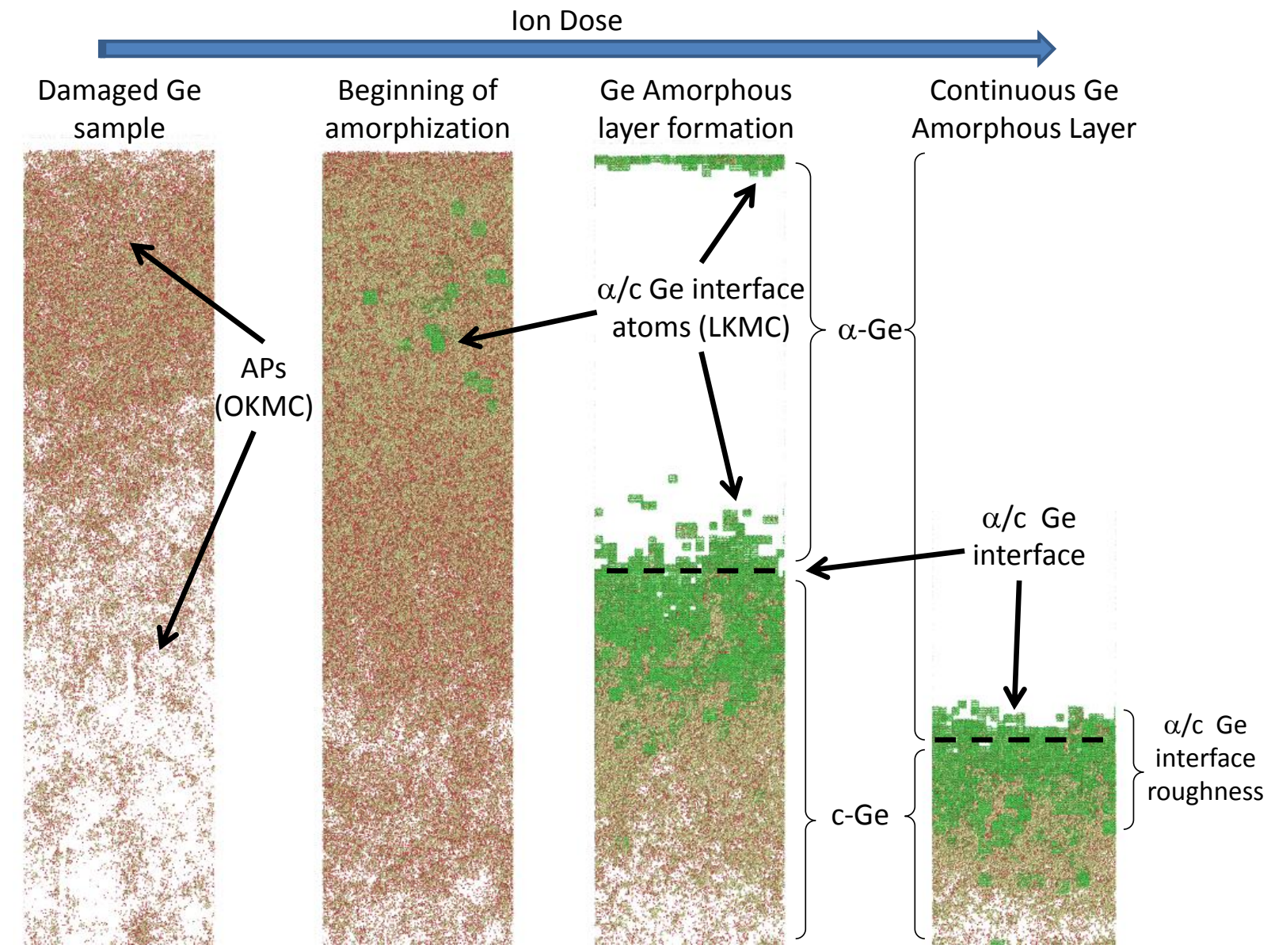

Figure 5.3: Amorphization process implemented in MMonCa. Images are taken from simulation of different doses of a Ge $150 \mathrm{keV}$ implant, introduced in OVITO, Stukowski (2010).

damage caused consists on the taken $I$ s and $V$ s from the BCA results, and associations of them, in the form of Amorphous Pockets, ( $A P \mathrm{~s}$, or $I V$ Clusters), $I$-type clusters or $V$-type clusters, namely Voids. When the introduced damage locally overcomes the amorphization threshold, that volume is amorphized, the material is changed into its amorphous phase, and the LKMC module is called to introduced the atoms of the $\alpha / c$ interface. When a surrounding box (or Mesh Element) is amorphized, those $\alpha / \mathrm{c}$ interface atoms are removed, and the new $\alpha / \mathrm{c}$ interface updated. As the dose increases, more amorphous regions take place, and very well defined $\alpha / \mathrm{c}$ interfaces appear.

Regarding identification of amorphous layers, it is a complex task to choose a consistent 
criterion. For instance, different models have been reported with different standards for $\mathrm{Si}$ amorphization modeling, Claverie et al. (1989); Vieu et al. (1992). In this model, we identify the local position of the transition between the two phases by a rigorous numerical criterion based on the mean value of the positions of the atoms placed at the local $\alpha / c$ interfaces. Of course, different buried amorphous layers are well differentiated in the process when more than one interface appears. This also provides a value of the simulated roughness of such interface, resulting from regions where both phases coexist.

Dynamic annealing events are modeled through recombination energies which depend on the damage configuration. Agglomeration of $I \mathrm{~s}$ and $V$ s give rise to compact damaged Ge regions of different sizes, namely $A P \mathrm{~s}$. The effective size, $s$, of an $A P$ formed by $n$ $I$ s and $m V \mathrm{~s}$ is computed as $s=\min (n, m)$. This definition accounts for the number of available $I V$-pairs inside an $A P$ able to perform the reaction $I+V \rightarrow 0$, Pelaz et al. (2004). For every recombination event, an $A P$ of size $s$ shrinks to $(s-1)$ at a rate of, Mok et al. (2008):

$$
\nu=\alpha s^{\beta} \exp \left[-E_{a c t}(s) / k T\right]
$$

where $\alpha=5 \times 10^{-3} \mathrm{~cm}^{2} \mathrm{~s}^{-1}, \beta=1$ and $E_{\text {act }}(s)$ is the activation energy of Ge $A P$ recombination shown in Fig. 5.4 (solid line). One immediate result of both this effective size definition and the proposed size dependency is that stability of these clusters may be thought as a "coordination" between the number of $I$ s and $V$ s. This means that an $I_{1} V_{9}(s=1)$ would recombine much easier than an $I_{5} V_{5}(s=5)$, even though both are composed by the same number of defects. In Fig. 5.4, energies reported for $A P$ recombination in $\mathrm{Si}$, Mok et al. (2008) are also presented. Further discussions on these energies and comparisons between both materials are given in Section 5.3 .2 .

Prefactors and energies for the Arrhenius expressions of migration and formation of $I \mathrm{~s}$ and $V$ s have been calibrated in agreement with previous studies, Chroneos and Bracht (2014); Vanhellemont et al. (2007); Śspiewak et al. (2008); Sueoka and Vanhellemont (2006) and are shown in Table 5.1 ,

A comprehensive discussion on the formation and binding energies of pure $I$ - and $V$ type clusters is out of the scope of this work. Nevertheless, a simple calibration has been performed in order to be able to reproduce some experimental results. This calibration is discussed in Section 5.3 .4 


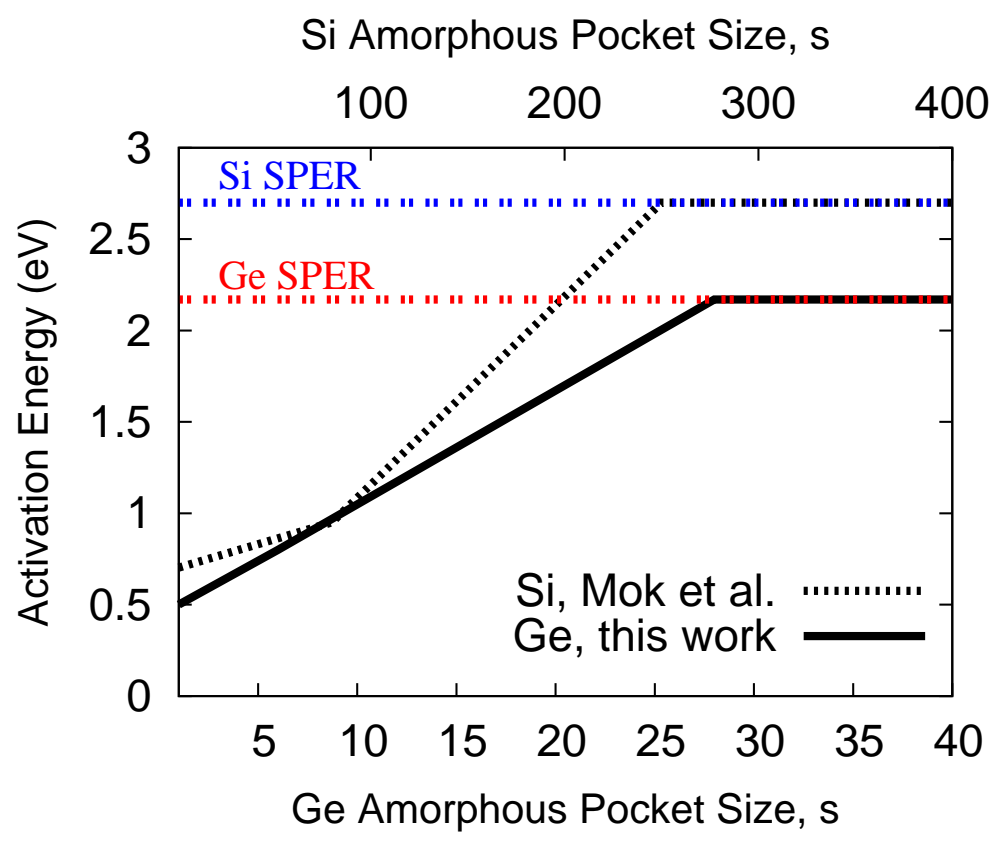

Figure 5.4: Activation energies of recombination as a function of the Amorphous Pocket effective size, $s$, for Si, Mok et al. (2008) (dashed line) and Ge, this work (solid line). SPER energies are also shown for Si, Olson and Roth (1988); Martin-Bragado and Moroz (2009) (blue) and Ge, Lu et al. (1990); Darby et al. (2013) (red).

Table 5.1: Migration and formation energies $\left(E_{m}, E_{f}\right)$ and prefactors $\left(D_{0, m}, D_{0, f}\right)$ of Ge $I \mathrm{~s}$ and $V \mathrm{~s}$ used in this model.

\begin{tabular}{lcccc}
\hline \hline & & & & \\
& $D_{0, m}\left(\mathrm{~cm}^{2} \mathrm{~s}^{-1}\right)$ & $E_{m}(\mathrm{eV})$ & $D_{0, f}\left(\mathrm{~cm}^{-3}\right)$ & $E_{f}(\mathrm{eV})$ \\
\cline { 2 - 5 }$I$ & $1 \times 10^{-5}$ & 0.8 & $1 \times 10^{28}$ & 3.75 \\
$V$ & 10 & 0.77 & $1 \times 10^{24}$ & 2.43 \\
\hline \hline
\end{tabular}

\subsection{Results and Discussion}

Damage accumulation by means of ion implantation, amorphizing or not, is a highly complex process in which several mechanisms take place. Thus, results are presented by separating the different stages of Ge amorphization. In Section 5.3.1, amorphization 
is discussed by analyzing the mechanisms taking place when dynamic annealing events can be neglected. In Section 5.3.2, dynamic annealing effects of defect recombination are studied. Section 5.3 .3 is focused on differences reported between ions, and their effects on damage generation and amorphization. Finally, in Section 5.3.4 mechanisms of damage dissolution are discussed.

Despite these results are separated in such way, all simulations have been performed using the same model and calibration.

\subsubsection{Amorphization threshold}

A $\langle 100\rangle$ germanium wafer with a resistivity in the $15-22 \Omega$ range was implanted at CEMES $/ \mathrm{CNRS}^{1}$ with $40 \mathrm{keV} 1 \times 10^{15} \mathrm{~B} / \mathrm{cm}^{2}$ ions at $100 \mathrm{~K}$. This temperature was chosen to minimize dynamic annealing events, Impellizzeri et al. (2011) focusing only on the pure collisional part of the damage buildup mechanisms towards amorphization. Then, the sample was prepared for transmission electron microscopy (TEM) according to the standard preparation procedure consisting of mechanical grinding and ion milling at the same temperature of implantation to minimize defect annealing. Cross sectional TEM (XTEM) observation of the sample at room temperature (RT) showed a continuous amorphous layer extending from the surface up to $\sim 135 \mathrm{~nm}$ (not shown). The temperature of analysis is not expected to produce any further impact in the position of the measured $\alpha / c$ interface position, once formed, since higher temperatures would be needed to induce the SPER process Lu et al. (1990); Claverie et al. (2010); Darby et al. (2013).

Simulating such conditions, without defining any amorphization threshold, a damage concentration of $\sim 1.3 \times 10^{22} \mathrm{~cm}^{-3}$ was obtained at the mentioned depth. The use of this value as the amorphization threshold, i.e. $\rho_{t} \simeq 1.3 \times 10^{22} \mathrm{~cm}^{-3}$, gave rise to an $\alpha / c$ transition at $(140 \pm 10) \mathrm{nm}$, in good agreement with the observation. This value of $\rho_{t}$ is also able to reproduce the $\alpha / c$ locations measured by Impellizzeri et al. (2009a) for $35 \mathrm{keV}$ B implants at liquid nitrogen temperature $\left(\mathrm{LN}_{2} \mathrm{~T}\right)$. These results, obtained for cryogenic conditions, are presented among others, for different experimental conditions, later in this work in

\footnotetext{
${ }^{1}$ Dr. Alain Claverie is deeply thanked for sharing such experimental data.
} 


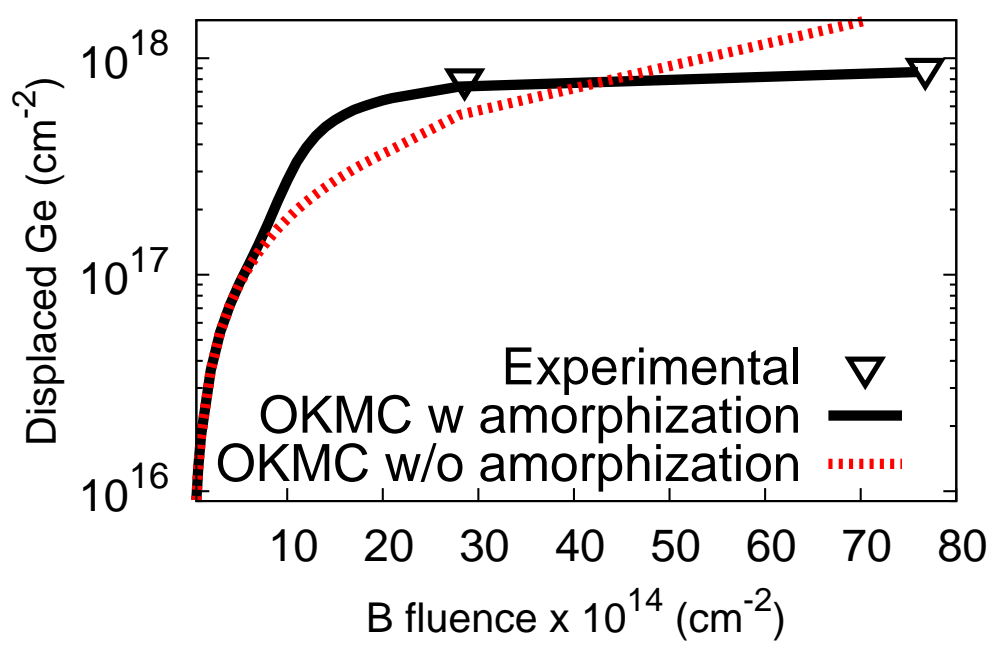

Figure 5.5: Displaced Ge atoms for different $35 \mathrm{keV}$ B fluences at $\mathrm{LN}_{2} \mathrm{~T}$. Symbols represent experimental data.Impellizzeri et al. (2009a) Simulation results with (solid line) and without (dashed line) the amorphization model are presented.

Fig. 5.12. It is important to notice that the presented value of $\rho_{t}$ does not depend on the implantation conditions.

In the mentioned work of Impellizzeri et al. (2009a) channeling Rutherford backscattering spectrometry (c-RBS) was used to determine the amount of damage caused in the Ge lattice after implantations. Such measurements resulted in the quantity of damage per unit of implanted area, integrated over the whole depth of the sample. We have analyzed those results together with our simulations in order to better understand the amorphization process of Ge.

Fig. 5.5 is a comparison between experimental, Impellizzeri et al. (2009a) (symbols) and simulated (lines) results of the displaced Ge atoms after $35 \mathrm{keV}$ B implants at $\mathrm{LN}_{2} \mathrm{~T}$ for different fluences. The dashed line represents simulation results without settling a value of $\rho_{t}$. It is observed that the damage increases without any limit, and no agreement could be found. These simulations do not either reproduce different implantation regimes, Decoster and Vantomme (2009). On the contrary, results obtained by applying the amorphization model (solid line) not only matches a perfect agreement, but also helped to elucidate the fundamental mechanisms of Ge amorphization. 


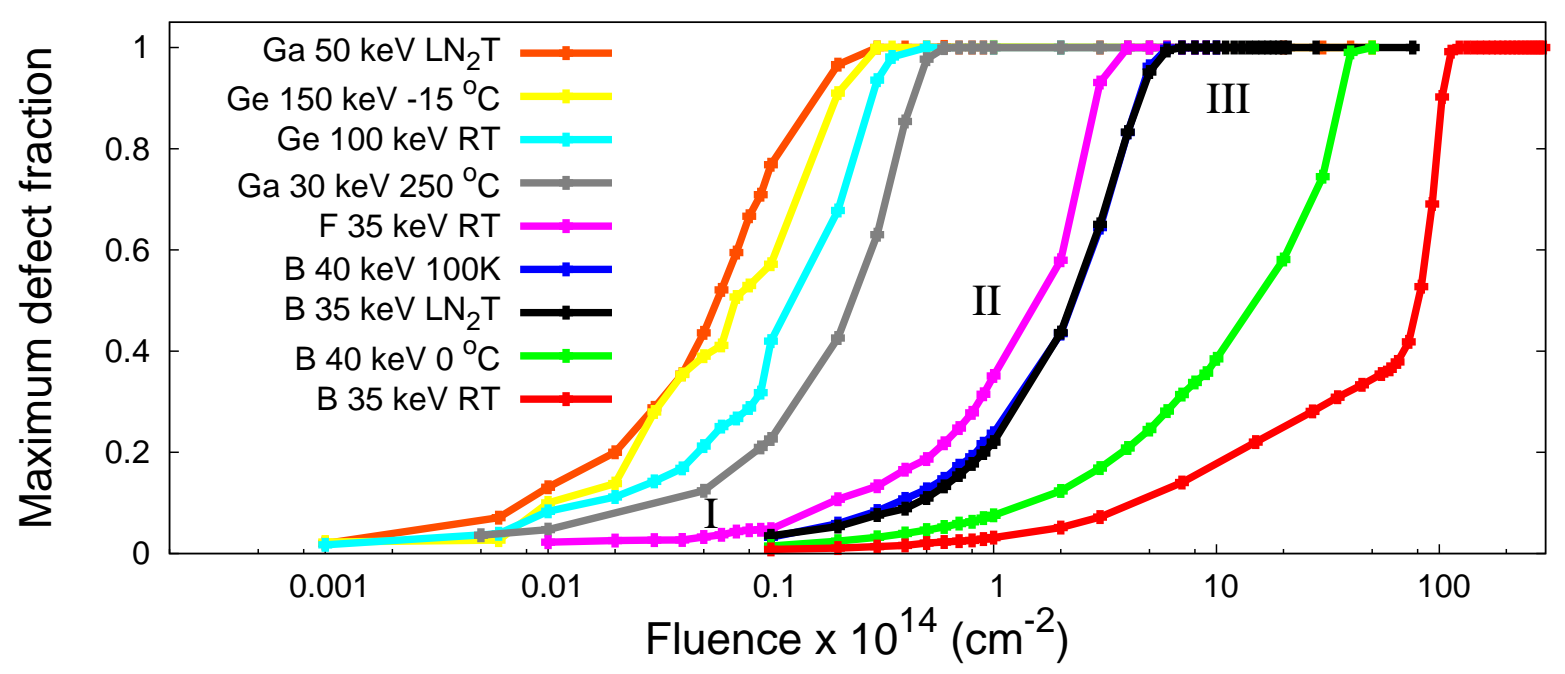

Figure 5.6: Maximum defect fraction for increasing fluences for all the ions studied in this work. The three different regions of damage accumulation are also shown.

As c-RBS measurements are able to extract the quantity of displaced Ge atoms (i.e. I atoms), a distinction between the different damage generation regimes can be made (roman numerals in Fig. 5.6). Damage is accumulated linearly until the amorphization threshold is locally exceeded, and that part of the lattice relaxes. This means that introducing $\sim 30 \%$ damage concentration of Ge atomic density, $\rho=4.42 \times 10^{22} \mathrm{~cm}^{-3}$, is enough to collapse the lattice structure of Ge. This results in a faster damage accumulation. At this point, there is a coexistence of amorphized regions with partially damaged ones, which continue collapsing into the amorphous phase as the fluence increase. When nearly the full depth of the damage profile has amorphized, no more damage can be generated, and the damage accumulation saturates for enough extended amorphous layers. This interpretation explains the measured values by c-RBS, their tendency, and the observed implantation regimes.

Moreover, by studying the maximum defect fraction in the implanted sample, three different regions of damage accumulation have been classically distinguished Bai and Nicolet (1991); Pelaz et al. (2004). Fig. 5.6 shows the maximum defect fraction achieved for increasing fluences for all the ions studied in this work. By analyzing the different growing velocities of the peaks of maximum damage, shown by the slopes of the curves, those three different regions of damage accumulation are extracted and shown in Table 5.2. In Region 
Table 5.2: $\quad c$ - to $\alpha$-cascade transitions used for all the studied conditions in this work, and transitions between the three different regions of damage accumulation in the peak of maximum damage, extracted from Fig. 5.6 (all means conditions used in this study).

\begin{tabular}{ccccc|cc}
\hline \hline Ion & $\begin{array}{c}\text { Energy } \\
(\mathrm{keV})\end{array}$ & $\begin{array}{c}\text { Temperature } \\
\left({ }^{\circ} \mathrm{C}\right)\end{array}$ & $\begin{array}{c}\text { Flux } \\
\left(\mathrm{cm}^{-2} \mathrm{~S}^{-1}\right)\end{array}$ & $\begin{array}{c}c \rightarrow \alpha \\
\left(\mathrm{cm}^{-2}\right)\end{array}$ & $\begin{array}{c}I \rightarrow I I \\
\left(\mathrm{~cm}^{-2}\right)\end{array}$ & $\begin{array}{c}I I \rightarrow I I I \\
\left(\mathrm{~cm}^{-2}\right)\end{array}$ \\
\hline $\mathrm{B}$ & 40 & -173 & $3 \times 10^{12}$ & full $c$ & $4 \times 10^{13}$ & $6 \times 10^{14}$ \\
$\mathrm{~B}$ & 40 & 0 & $2.3 \times 10^{12}$ & full $c$ & $1 \times 10^{14}$ & $4 \times 10^{14}$ \\
$\mathrm{~B}$ & 35 & 20 & all & full $c$ & $3 \times 10^{14}$ & $1 \times 10^{16}$ \\
$\mathrm{~B}$ & 35 & -196 & $3 \times 10^{12}$ & $2.8 \times 10^{15}$ & $4 \times 10^{13}$ & $6 \times 10^{14}$ \\
$\mathrm{~F}$ & 35 & 20 & $1 \times 10^{12}$ & full $\alpha$ & $1 \times 10^{13}$ & $4 \times 10^{14}$ \\
$\mathrm{Ge}$ & 100 & 20 & $1 \times 10^{12}$ & full $\alpha$ & $5 \times 10^{11}$ & $4 \times 10^{13}$ \\
$\mathrm{Ge}$ & 150 & -15 & all & full $\alpha$ & $5 \times 10^{11}$ & $3 \times 10^{13}$ \\
$\mathrm{Ga}$ & 30 & 20 & $1 \times 10^{19}$ & full $\alpha$ & $6 \times 10^{11}$ & $2 \times 10^{13}$ \\
$\mathrm{Ga}$ & 30 & 20 & $1 \times 10^{12}$ & full $\alpha$ & $1 \times 10^{12}$ & $3 \times 10^{13}$ \\
$\mathrm{Ga}$ & 30 & 250 & $1 \times 10^{19}$ & full $\alpha$ & $1 \times 10^{12}$ & $5 \times 10^{13}$ \\
$\mathrm{Ga}$ & 30 & 250 & $1 \times 10^{12}$ & full $c$ & - & - \\
$\mathrm{Ga}$ & 50 & -196 & $1 \times 10^{12}$ & full $\alpha$ & $5 \times 10^{11}$ & $3 \times 10^{13}$ \\
& & & & & \\
\hline \hline
\end{tabular}

I, damage is produced slowly until the threshold is overcome. In Region II, there is a dramatic increase in its production due to the relaxation to the $\alpha$-Ge phase. These areas nucleate until a full layer is amorphized and, as the lattice has relaxed, no more damage at the peak can be generated. Thus, the damage production saturates at that layer to 1 , placing the accumulation into the Region III, where only widening of the layer takes place.

This behavior has already been widely studied in Si, Pelaz et al. (2004). As we consider only a structural phase transition without dynamic effects, and the fact that $\mathrm{Si}$ and $\mathrm{Ge}$ lattices are similar, the model can be thought to be applicable to both Si and Ge materials. Mentioned tendencies and results for c-RBS measurements are also observed for other 
conditions and compared with our results later in this work.

The presented threshold value of amorphization in terms of damage concentration, $\rho_{t} \simeq 1.3 \times 10^{22} \mathrm{~cm}^{-3}$, can now be compared with previous works. Koffel et al. (2009) used the CDED model to determine the energy needed to amorphize Ge with $150 \mathrm{keV}$ Ge ions at $-15{ }^{\circ} \mathrm{C}$, while Impellizzeri et al. (2011) pointed out the difference in that critical energy density, $E_{d c}$, between implantations at $\mathrm{LN}_{2} \mathrm{~T}$ and RT. Despite both Koffel et al. (2009) and Impellizzeri et al. (2011) agree to a value of $E_{d c} \simeq 5 \mathrm{eV} /$ atom for temperatures of $-15^{\circ} \mathrm{C}$ or $\mathrm{RT}$, the last cited work also stated that only $\sim 1.3 \mathrm{eV} /$ atom are needed to amorphize the Ge sample at cryogenic conditions.

In order to compare these values against our model, these discrepancies deserve further explanations. The value of $E_{d c}$ represents the quantity of energy needed to be deposited along the sample to achieve amorphization, providing an estimation of the extension of amorphous layers in which the deposited energy has overcome that value. In that sense, both the CDED and the presented model are similar, since in our model that energy is evaluated in terms of generated defects. Nevertheless, as shown in the work of Impellizzeri et al. (2011) different values of $E_{d c}$ are achieved for different temperatures. This is due to the fact that, for increasing temperatures, the deposited energy is "absorbed" through Frenkel Pair recombinations. These recombinations are taken into account in this work as explained in Section 5.2, and further discussed in Section 5.3.2. Considering the threshold displacement energy per atom, $E_{t}$, (i.e. the energy needed to displace an atom from its lattice position) used in this model of $30 \mathrm{eV} /$ atom and the density of Ge, a comparison between both concentration and energy thresholds can be done by following the relation:

$$
E_{d c}=E_{t} \times \frac{\rho_{t}}{2 \rho}
$$

which results in $E_{d c} \simeq 4.4 \mathrm{eV} /$ atom. The factor 2 in this expression is an approximation to consider only displaced $I \mathrm{~s}$, and not $V \mathrm{~s}$. On the one hand, this result is lower than the reported $\sim 5 \mathrm{eV} /$ atom in Refs. Koffel et al. (2009); Impellizzeri et al. (2011), where it can be considered that at $-15^{\circ} \mathrm{C}$ (or RT) there is a loss of energy spent in dynamic annealing effects. On the other hand, it is also higher than the presented by Impellizzeri et al. (2011) of $\sim 1.3 \mathrm{eV} /$ atom for $\mathrm{LN}_{2} \mathrm{~T}$ conditions. Considering the strong dependence in the initial damage calculations for both models and the high differences in the values of 
$E_{t}$, (15 against the $30 \mathrm{eV} /$ atom used in or calculations) we can conclude that the presented value of $\rho_{t} \simeq 1.3 \times 10^{22} \mathrm{~cm}^{-3}$ is consistent.

\subsubsection{Dynamic annealing}

Once the amorphization threshold was chosen, we considered dynamic events, which occur at higher temperatures, causing a competition between damage generation and its dynamic annealing, Posselt et al. (2006). Such events are modeled through the AP recombination energies already shown in Fig. 5.4. Ge recombination energies start at $\sim 0.5 \mathrm{eV}$ for the smallest sizes and grow up to the activation energy of Solid Phase Epitaxial Regrowth (SPER) of $2.17 \mathrm{eV}$ for a planar $\alpha / \mathrm{c}$ interface, Lu et al. (1990); Darby et al. (2013); GomezSelles et al. (2014). This approach has already been validated for Si in both continuous and atomistic models, Morehead Jr and Crowder (1970); Mok et al. (2008); Pelaz et al. (2004).

It is noticeable that Ge recombination energies presented in this work are generally lower than those reported for Si, Mok et al. (2008). This is connected to the fact that, in Ge, damage recovery occurs at lower temperatures than in Si, Satta et al. (2005). To better clarify this statement, and to evaluate the influence of different prefactors in equation 5.1 . we have studied the differences between calculated total rates for two different temperatures. Fig. 5.7 is a comparison between Si, Mok et al. (2008) and Ge AP recombination rates as a function of their effective size, $s$, at both $300 \mathrm{~K}$ and $500 \mathrm{~K}$. AP recombination rates are generally orders of magnitude lower in Si than in Ge. This means that for a given time, temperature and $A P$ distribution, much more recombination events will take place in $\mathrm{Ge}$ than in Si. It is worth mentioning that for Ge, these clusters of small sizes have been able to explain the fundamental processes of damage buildup, when comparing with the larger reported sizes in Si.

\subsubsection{Differences between ions}

\section{Damage quantity and distribution for c- and $\alpha$-cascades}

As it has already been mentioned in Section 5.2 , damage cascades are obtained in two different sets of simulations: $\alpha$-cascades assuming an amorphous target, and $c$-cascades 


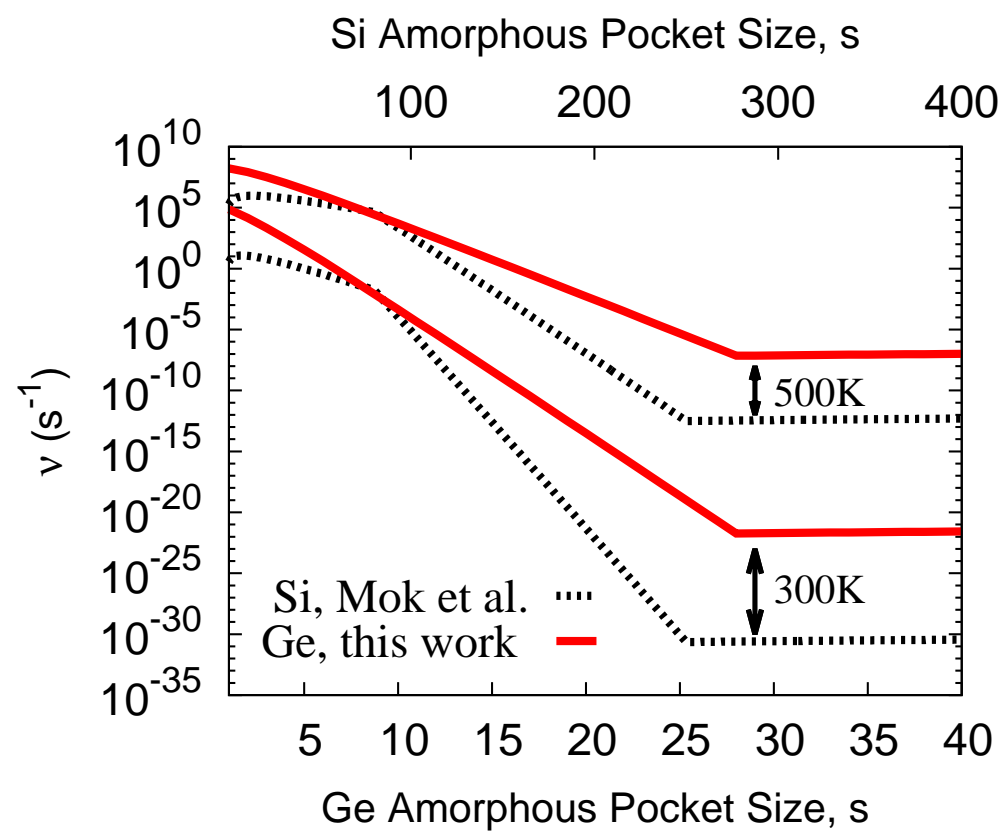

Figure 5.7: $A P$ recombination rates, $\nu$, as a function of their effective size, $s$, for $\mathrm{Si}$, Mok et al. (2008) (dashed line) and Ge, this work (solid line), for both 300K and 500K. Corresponding temperatures are indicated with arrows between Si and Ge lines.

assuming a pure crystalline target. For all the studied conditions, 3000 damage cascades were calculated for both type of cascades. In this Section, the differences between both sets of calculations are studied.

Fig. 5.8 is a comparison of the damage profiles calculated BCA assuming an amorphous (blue) or a crystalline target (red) for (a) $35 \mathrm{keV}, 1 \times 10^{14} \mathrm{~B} / \mathrm{cm}^{-2}$ (b) $30 \mathrm{keV}, 2.5 \times 10^{13}$ $\mathrm{F} / \mathrm{cm}^{-2}$ (c) $30 \mathrm{keV}, 1 \times 10^{13} \mathrm{Ga} / \mathrm{cm}^{-2}$ and (d) $150 \mathrm{keV}, 5 \times 10^{12} \mathrm{Ge} / \mathrm{cm}^{-2}$. In all cases, there is a non-negligible difference in terms of damage quantity and distribution when comparing $c$ - and $\alpha$-cascades. These differences are that: i) the quantity of damage produced when assuming an amorphous target, specially in the area of maximum damage generation (or energy deposition) is significantly higher than when assuming a crystalline target, and ii) the damage distribution is also different. This last difference relies on the typical tail of channeled implantations, as expected, since as explained in Chapter 4 the only difference between both sets of calculations is the assumption of an homogeneous distribution ( $\alpha$-cascades) versus a perfect crystalline lattice ( $c$-cascades). 


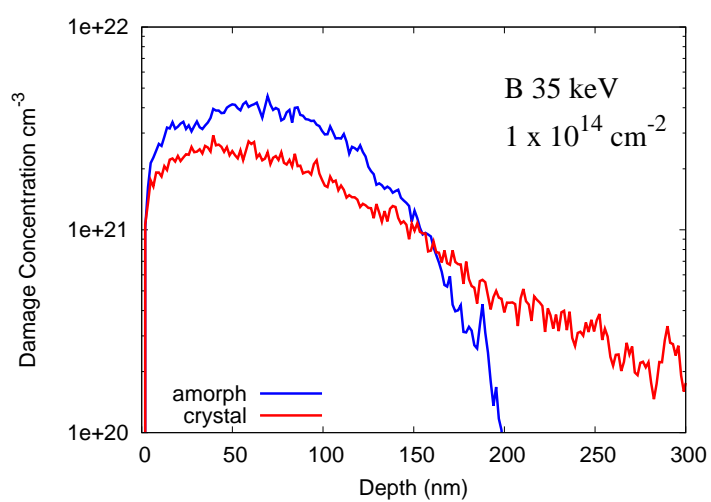

(a)

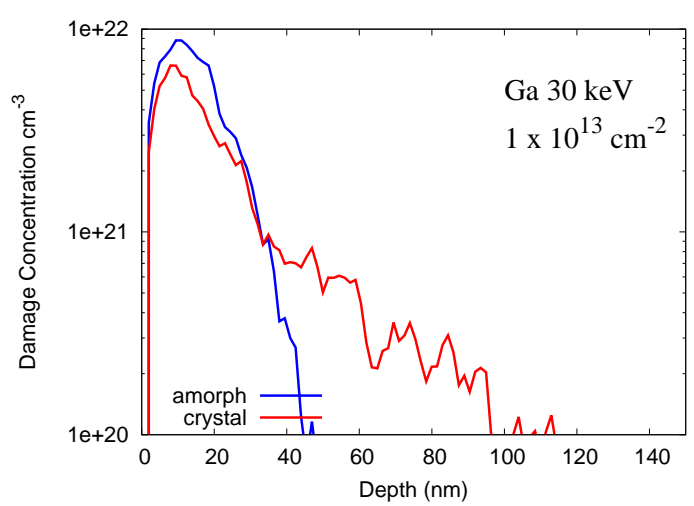

(c)

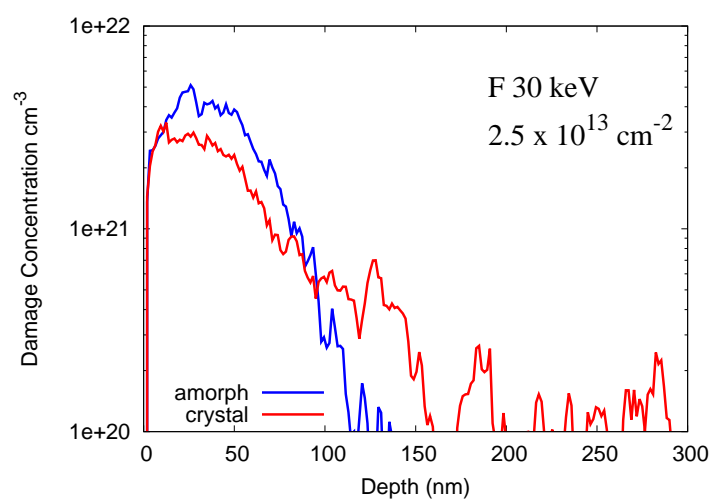

(b)

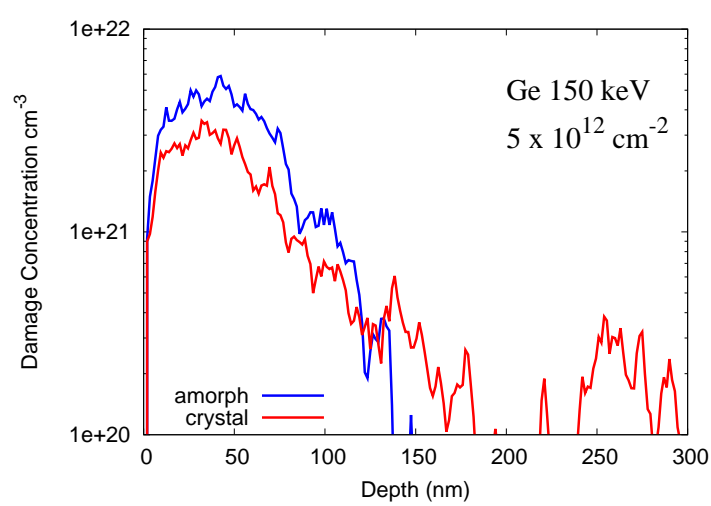

(d)

Figure 5.8: Comparison of the damage profiles calculated with BCA assuming an amorphous (blue) or a crystalline target (red) for (a) $35 \mathrm{keV}, 1 \times 10^{14} \mathrm{~B} / \mathrm{cm}^{-2}$ (b) $30 \mathrm{keV}$, $2.5 \times 10^{13} \mathrm{~F} / \mathrm{cm}^{-2}$ (c) $30 \mathrm{keV}, 1 \times 10^{13} \mathrm{Ga} / \mathrm{cm}^{-2}$ and (d) $150 \mathrm{keV}, 5 \times 10^{12} \mathrm{Ge} / \mathrm{cm}^{-2}$.

To evaluate quantitatively the differences between both sets of cascades, simulations of the conditions presented in Fig. 5.8 plus $40 \mathrm{keV}, 1 \times 10^{14} \mathrm{~B} / \mathrm{cm}^{-2}$ and $100 \mathrm{keV}, 5 \times 10^{12}$ $\mathrm{Ge} / \mathrm{cm}^{-2}$ (i. e. all the studied ions and energies in this work) were carried out. In such simulations, the cascades were picked randomly from those $\sim 3000$ at each (c- or $\alpha-)$ condition until the desired dose was achieved, to extract the damage concentration induced by the implantation. Fig. 5.9 shows the ratios between the damage concentration obtained for both conditions, after repeating the process five times with five different random seeds and extracting the geometric mean. Despite the ratios are very different 
when comparing the different ions and energies $(\sim 1.16$ for $30 \mathrm{keV} \mathrm{Ga}$ ions against $\sim 1.48$ for $150 \mathrm{keV}$ Ge ions) there is not a clear evidence of a systematic dependence of the ion mass on such damage quantity difference. Thus, the only conclusion that can be obtained from these considerations is that the damage production calculated assuming an atomistic randomly distributed target is higher than when assuming a perfect crystalline lattice. It is worth noting that (as shown in Fig. 5.8, in logarithmic scale) this damage difference is centered in the peak of maximum energy deposition (or damage generation). For this reason, choosing between using $c$ - or $\alpha$-cascades will have a high impact on the final results. As it will now be discussed, this will add a limitation to the model, making it necessary to establish a criterion in the use of each type of cascades.

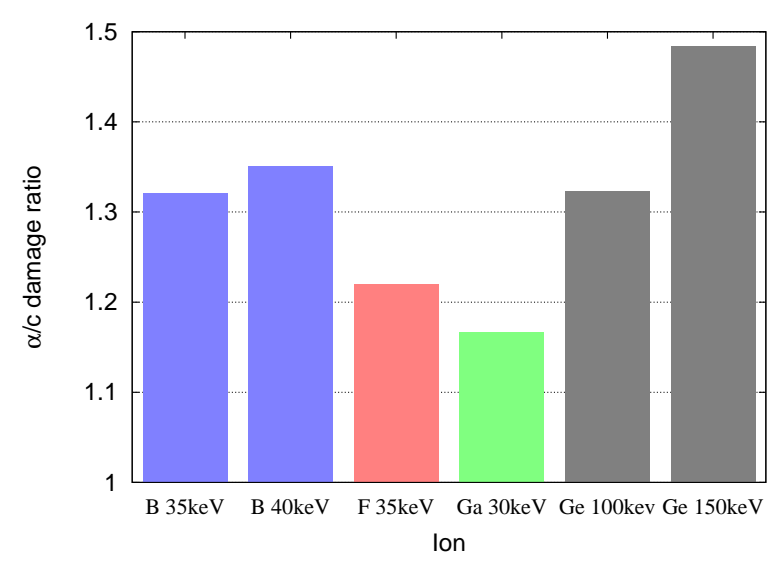

Figure 5.9: $\alpha /$ c damage ratios for the studied ions.

In fact, in this work, amorphization is studied from point defect generation, and up to amorphization of full extended layers of Ge under ion implantation. This comprises effects of dynamic annealing, damage accumulation and partial amorphization of the Ge target. If, on the one hand, the BCA simulator takes into account the differences of the ion mass in the channeling effects, on the other hand de-channeling is not taking into account. After calibration of the model, it has been found that, while for B ions, $c$-cascades are needed to be used until the damage production saturates, for the rest of ions (higher mass), full $\alpha$-cascades are simulated, as shown in Table 5.2, except for the case of the Ga implant at $250{ }^{\circ} \mathrm{C}$ at $10^{12} \mathrm{~cm}^{-2} \mathrm{~s}^{-1}$ as discussed on page 90 . 
(a)

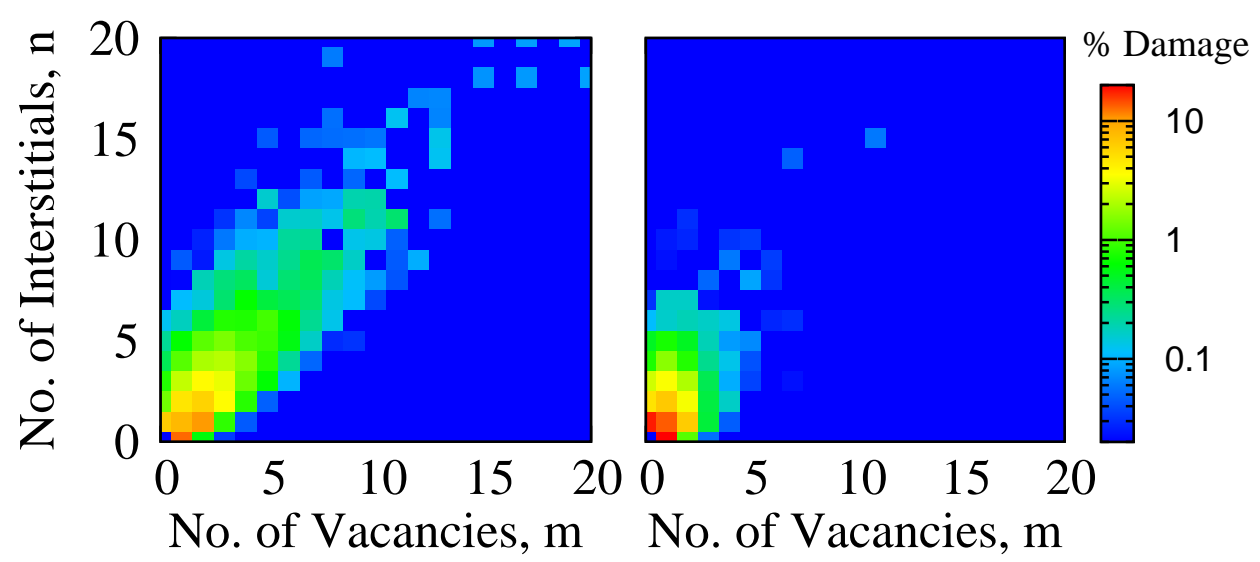

(c)

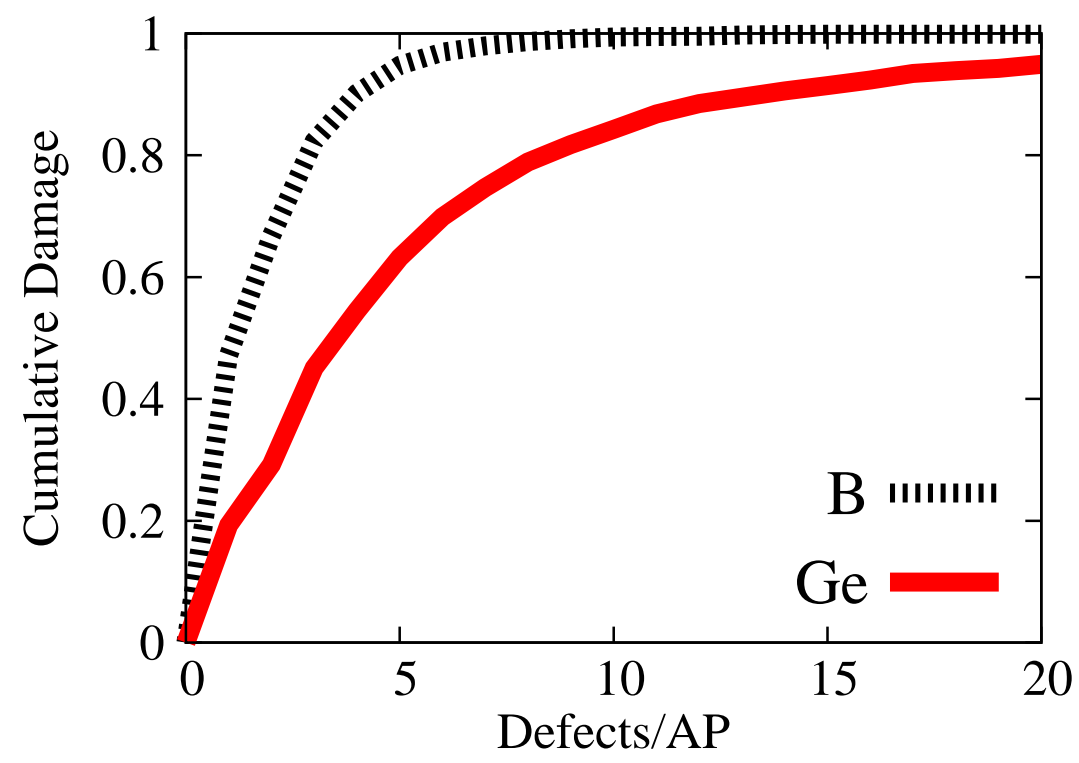

Figure 5.10: 2D Concentration distribution histogram of $A P$ composition, $I_{n} V_{m}$, resulting after simulations of $150 \mathrm{keV}$ Ge (a) and $40 \mathrm{keV} \mathrm{B}$ (b) implants at $\mathrm{LN}_{2} \mathrm{~T}$. (c) is the normalized cumulative damage distribution of the same data for both Ge (solid line) and $\mathrm{B}$ (dashed line) implantation as a function of the number of defects forming an $A P$. Fluences where chosen to generate the same amount of damage ( $I \mathrm{~s}$ and $V \mathrm{~s}$ ) for both conditions. 


\section{Damage coordination}

It has been reported that the damaged regions caused by $\mathrm{B}$ and other light ions are smaller and more diluted than by heavier ions, Koffel et al. (2009); Napolitani et al. (2014). To study the possible variations between different ion masses, simulations of B and Ge implantations were performed. Fig. 5.10 shows the 2D concentration distribution histogram for $A P$ s resulting from simulations of $150 \mathrm{keV}$ Ge (a) and $40 \mathrm{keV} \mathrm{B} \mathrm{(b)} \mathrm{implants} \mathrm{at} \mathrm{LN}_{2} \mathrm{~T}$, by using $c$-cascades. The color represents the percent contribution to the total damage of every $A P$, formed by $n I \mathrm{~s}$ (y-axes) and $m V \mathrm{~s}$ (x-axes). Fluences where chosen to generate $\sim 22000 I$ s and $V$ s at both conditions. The low temperature was again chosen to study the damage composition without further dynamic annealing effects. APs resulting from Ge implantations consist of higher number of $I \mathrm{~s}$ and $V \mathrm{~s}$ than those generated by $\mathrm{B}$. In Fig. 5.10 (c) the normalized cumulative damage is presented for those Ge (solid line) and B (dashed line) implants. While $\sim 90 \%$ of the total damage caused by B ions is constituted by $A P$ s formed by less than 5 defects, higher number of defects per $A P(\sim 20)$ are needed to reach the same contribution to the total damage for a Ge implant. This examination suggests that the damage caused by Ge ions is more compact than the one caused by $\mathrm{B}$ ions.

To evaluate the impact of the presented recombination rates in the damage distribution caused by both $\mathrm{B}$ and Ge ions, we have made a comparison of the simulated induced damage by $40 \mathrm{keV}, 5 \times 10^{14} \mathrm{~B} / \mathrm{cm}^{2}$ and $150 \mathrm{keV}, 2.25 \times 10^{13} \mathrm{Ge} / \mathrm{cm}^{2}$ implants at both $\mathrm{LN}_{2} \mathrm{~T}$ and $0^{\circ} \mathrm{C}$, presented in Fig. 5.11. Such comparison shows that the annealed damage after implanting $\mathrm{Ge}$ ions at $0^{\circ} \mathrm{C}$ is much lower than that observed for $\mathrm{B}$ ions. Despite a high amount of damage is produced by $\mathrm{B}$ ions at $\mathrm{LN}_{2} \mathrm{~T}$, it is readily annihilated at relatively low temperatures. This description is able to explain the narrowness of the $\alpha$-Ge layers reported for B implants, Koffel et al. (2009).

This model has been able to reproduce $\alpha / c$ interface locations for a wide range of conditions. Fig. 5.12 is a comparison between experimentally measured and predicted $\alpha / c$ interfaces for different experimental measurements found in the literature for B, Koffel et al. (2009); Impellizzeri et al. (2009a) F, Boninelli et al. (2012b), Ga Impellizzeri et al. (2009b) and Ge, Koffel et al. (2009); Boninelli et al. (2012b); Satta et al. (2005) ions. Other conditions, for which other mechanisms take place will be discussed in Section 5.3.4. 


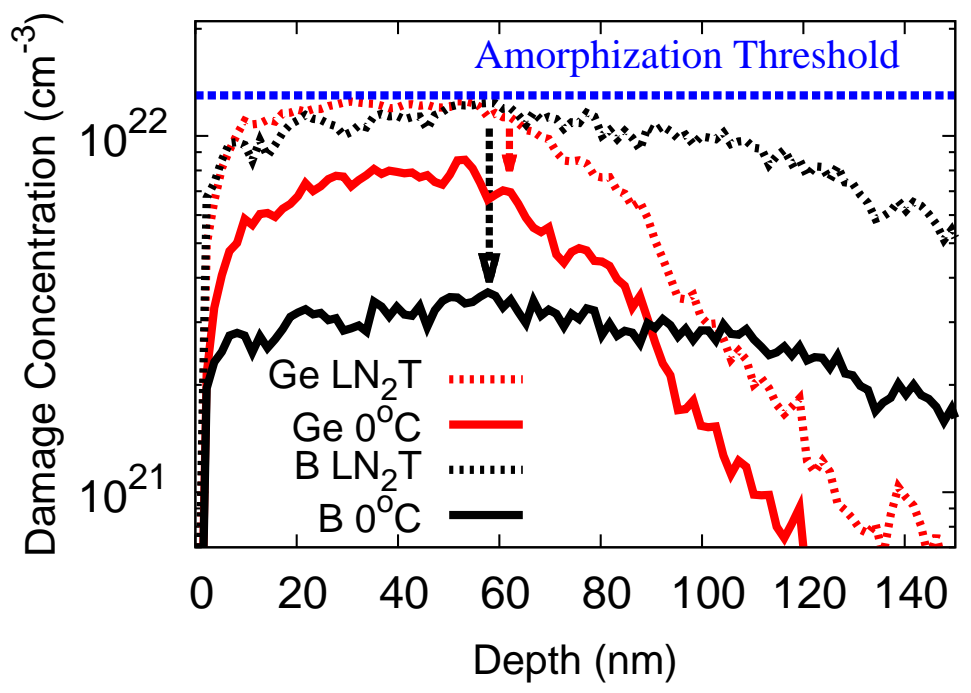

Figure 5.11: Damage profiles caused by $150 \mathrm{keV} 2.25 \times 10^{13} \mathrm{Ge} / \mathrm{cm}^{2}$ (red lines) and $40 \mathrm{keV} 5 \times 10^{14} \mathrm{~B} / \mathrm{cm}^{2}$ implants (black lines) at both $\mathrm{LN}_{2} \mathrm{~T}$ and $0^{\circ} \mathrm{C}$. Vertical arrows show the quantity of dynamically annealed damage when comparing the cryogenic implantations with those performed at $0^{\circ} \mathrm{C}$. The amorphization threshold is also represented (blue dashed line).

Uncertainty in the measurements (when provided) is shown by bars in the y-axis, while error bars in the predicted results stand for simulated roughness. The consonance between these results and reported observations, in this work and in the literature, supports the followed approach. In cases for which neither channeling nor dynamic annealing take place, this and the CDED model (see Ref. Koffel et al. (2009)) converge to similar results, as the approach taken is qualitatively similar for such conditions (i.e. the damage is accumulated until it overcomes a certain threshold, for which the $c-\alpha$ relaxation occurs). Otherwise our results are in a very good agreement with different experiments reported in the literature, shown by the proximity of the points to the dashed line. Agreement between experimental results for B, F, Ga and Ge implants and our simulations serves as a validation of the presented model. 


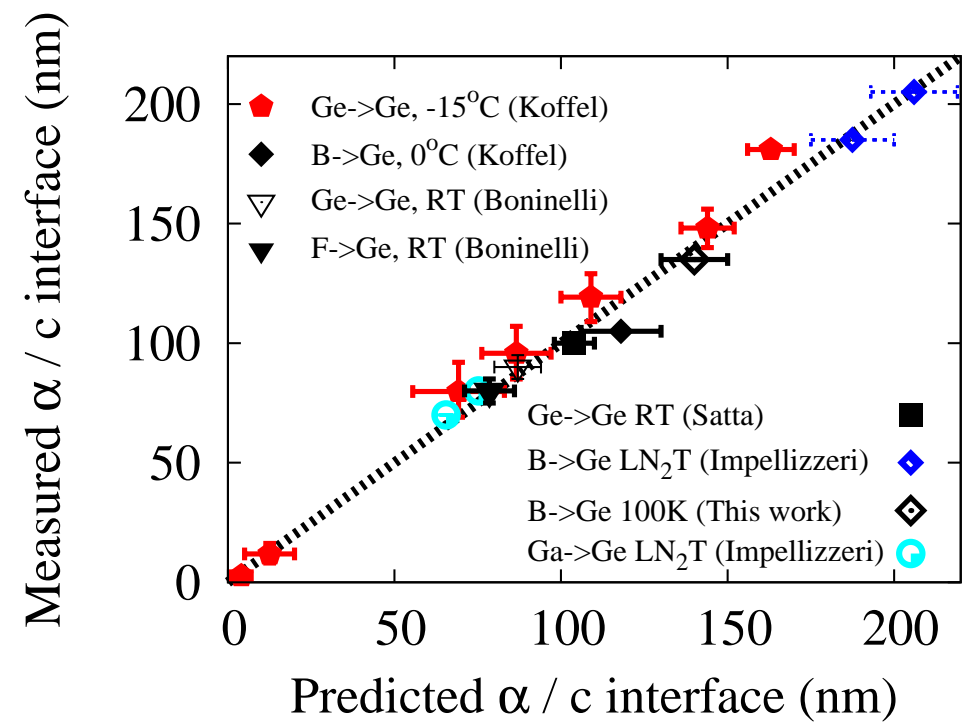

Figure 5.12: Location of $\alpha / c$ interfaces measured experimentally (y-axis) for $150 \mathrm{keV} \mathrm{Ge}$ implantations at $-15^{\circ} \mathrm{C}$ at different fluences, Koffel et al. (2009) $100 \mathrm{keV} 2 \times 10^{14} \mathrm{Ge} / \mathrm{cm}^{2}$ at RT, Boninelli et al. 2012b) $35 \mathrm{keV} 1 \times 10^{15} \mathrm{~F} / \mathrm{cm}^{2}$ at RT, Boninelli et al. 2012b) 100 $\mathrm{keV} 1 \times 10^{15} \mathrm{Ge} / \mathrm{cm}^{2}$ at RT, Satta et al. 2005) $40 \mathrm{keV} 5 \times 10^{15} \mathrm{~B} / \mathrm{cm}^{2}$ at $0^{\circ} \mathrm{C}$, Koffel et al. (2009) $35 \mathrm{keV} \mathrm{B}$ at $\mathrm{LN}_{2} \mathrm{~T}$ for different fluences, Impellizzeri et al. (2009a) $40 \mathrm{keV} 1 \times 10^{15}$ $\mathrm{B} / \mathrm{cm}^{2}$ at $100 \mathrm{~K}$ of this work, and $50 \mathrm{keV} 1 \times 10^{15} \mathrm{Ga} / \mathrm{cm}^{2}$ and $5 \times 10^{15} \mathrm{Ga} / \mathrm{cm}^{2}$ at $\mathrm{LN}_{2} \mathrm{~T}$, Impellizzeri et al. (2009b). Simulated $\alpha / c$ locations are represented in $\mathrm{x}$-axis.

\subsubsection{Cluster dissolution}

Results for damage accumulation towards amorphization have been presented so far for conditions in which only structural processes and/or dynamic annealing through $A P$ recombination are dominant. Nevertheless, for conditions of higher temperatures and/or low fluxes, we have investigated that other mechanisms as cluster dissolution can serve as an explanation for reported experiments Impellizzeri et al. (2009a); Posselt et al. (2006); Claverie et al. (2010); Boninelli et al. (2012a).

Fig. 5.13 shows a comparison between experimental, Impellizzeri et al. (2009a) measured by means of c-RBS techniques, (symbols) and simulation results (lines) of the concentration per unit area of implantation of $I$ s generated by $35 \mathrm{keV}$ B ions implanted at RT. With the model and calibration presented so far, agreement between experimental measurements and simulations could not be achieved (black dashed line). By investigating 
the damage left after those simulations, nearly only pure $I$ - and $V$-type stable clusters were present, evidencing that other physical mechanisms were needed to reproduce those results.

On the one hand, binding energies starting at $\sim 0.26 \mathrm{eV}$ where needed to activate $I$ and $V$ emissions in our simulations at these conditions. On the other hand, Sueoka et al. reported, by using $a b$ initio techniques, a binding energy for di-vacancies in Ge of $E_{b}\left(V_{2}\right)=$ $0.58 \mathrm{eV}$ and a vacancy formation energy of $E_{f}(V)=2.56 \mathrm{eV}$. Di-vacancy formation energy for those results can be calculated as $E_{f}\left(V_{2}\right)=2 \times E_{f}(V)-E_{b}\left(V_{2}\right)=4.54 \mathrm{eV}$, and $4.6 \mathrm{eV}$ for our parametrization (see table 5.1). These results enabled us to identify the mechanisms starting to appear at these conditions.

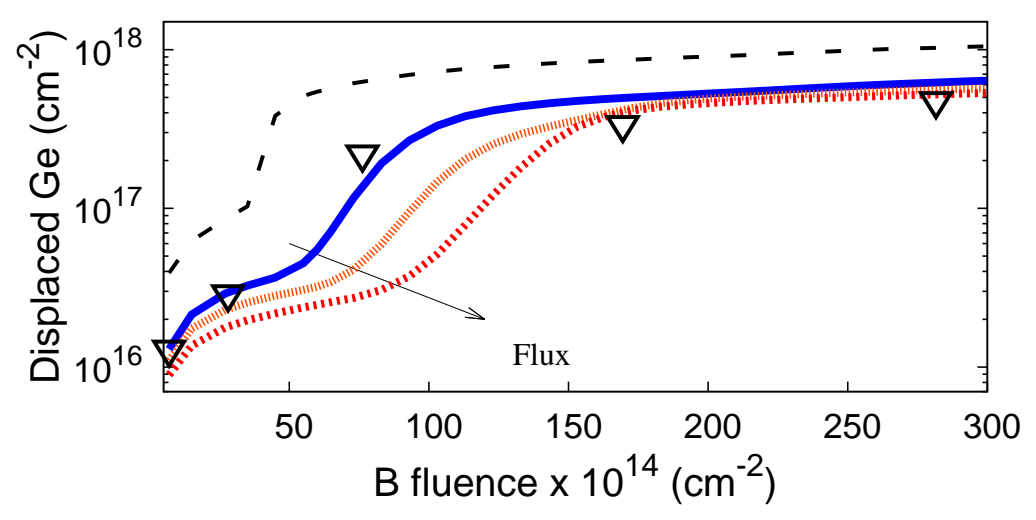

\section{No Cluster Dissolution - - \\ $6.24 \times 10^{11} \mathrm{~cm}^{-2} \mathrm{~s}^{-1}$ \\ $3.2 \times 10^{11} \mathrm{~cm}^{-2} \mathrm{~s}^{-1}$ \\ $1.75 \times 10^{11} \mathrm{~cm}^{-2} \mathrm{~s}^{-1}$ \\ Experimental $\nabla$}

Figure 5.13: Displaced Ge atoms for different $35 \mathrm{keV}$ B fluences at RT. Symbols represent experimental data, Impellizzeri et al. (2009a). Simulation results (lines) for different fluxes are also presented along with results obtained without cluster dissolutions.

Regarding binding and formation energies of the rest pure $V$ - or $I$-type clusters, we have performed a simple calibration in order to fit experimental results. Binding energies are interpolated for both type of clusters from the mentioned $0.26 \mathrm{eV}$ for $E_{b,\{n, m\}=2}=0.26 \mathrm{eV}$, and up to $E_{b,\{n, m\}=50}=1.2 \mathrm{eV}$. Even though a more systematic study on cluster binding and formation energies should be carried out in order to clearly identify these energies, (for example, by analyzing end of range defects, EOR, dissolution experiments, Satta et al. (2005); Claverie et al. (2010); Boninelli et al. (2012a)) this simple calibration has been able to achieve a good agreement between experimental results and our simulations. 
Due to the number of mechanisms that start to take place at these conditions, and their complexity, knowledge on the flux is extremely important in order to be able to perform realistic simulations and identify the underlying processes. Conversion between current density, often expressed in $\mu \mathrm{A} / \mathrm{cm}^{2}$, and ion flux is not always straightforward and can even depend on the specific experimental setup. In Ref. [Impellizzeri et al. (2009a)] an average current density of $\sim 1 \mu \mathrm{A} / \mathrm{cm}^{2}$ is reported. To analyze the impact of changing dynamic annealing times at these conditions, we have performed simulations for a set of three different fluxes of $\mathrm{B}$ ions, which are presented in Fig. 5.13. It can be observed that an overall reasonable match for the amount of generated damage can be achieved for variable fluxes.

Comparing these results with those at $\mathrm{LN}_{2} \mathrm{~T}$ in Fig. 5.5, the drop in the damage concentration is again noticeable, specially at the first stages of the implantation. This shows, jointly with the studies of Impellizzeri and co-workers, Impellizzeri et al. (2009a) the amount of damage that is annihilated at RT for B implantations in Ge. In the simulation results shown in Fig. 5.5 damage saturation is rapidly achieved. On the contrary, for implantations at RT (Fig. 5.13) the damage does not saturate completely for high fluences, and only $c$-cascades have been used. This can be linked to the fact that no $\alpha$-Ge continuous layers where experimentally observed. Nevertheless, our model shows formation of amorphizing areas, which is noticeable at the dramatic increase for the third experimental point. Moreover, in the experimental work it was reported that a buried damaged region was formed when B fluence exceeded $7.6 \times 10^{15} \mathrm{~B} / \mathrm{cm}^{2}$, in a very good agreement with this observation in our simulations. We have also observed in our simulations the formation of $\alpha$-Ge continuous layers for the two highest experimentally reported fluences at RT of $\sim 20 \mathrm{~nm}$ and $\sim 100 \mathrm{~nm}$, respectively to the last two points in Fig. 5.13. However, as it can be observed, even though the damage accumulation is not fully saturated, damage quantity is starting to stabilize. This may serve as an indicator of that amorphization of the target might start for slightly higher fluences. Moreover, for the same quantity of damage in the $\mathrm{LN}_{2} \mathrm{~T}$ case a large continuous amorphous layer is formed.

Fig. 5.14 is a comparison between experimental measurements, Posselt et al. (2006) (symbols) of relative disorder induced in the Ge sample by $30 \mathrm{keV} \mathrm{Ga}$ implantations for different fluences and ion fluxes at RT and $250^{\circ} \mathrm{C}$, and our simulations (lines). In this case, 


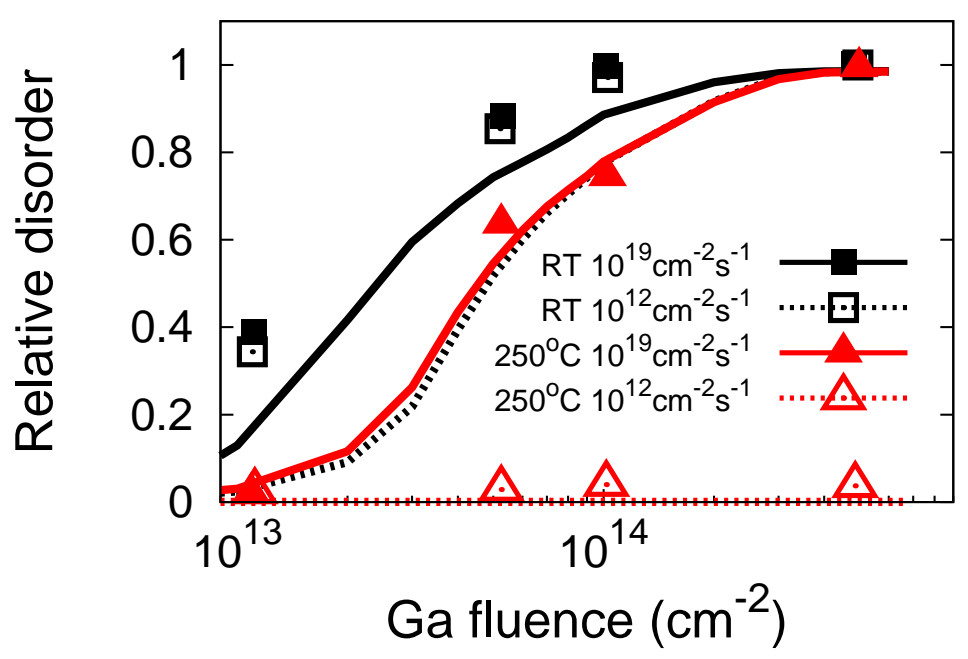

Figure 5.14: Experimental measurements, Posselt et al. 2006) (symbols) and simulated (lines) of the relative disorder induced in the Ge lattice after $30 \mathrm{keV}$ Ga implantations at both RT and $250^{\circ} \mathrm{C}$ for both $10^{12} \mathrm{~cm}^{-2} \mathrm{~s}^{-1}$ and $10^{19} \mathrm{~cm}^{-2} \mathrm{~s}^{-1}$ fluxes.

one should be cautious when doing quantitative comparisons between these c-RBS measurements and our simulations due to the possible different scattering efficiencies between the He ions and the type of defects existing when measuring, Posselt (2015). In order to compare these c-RBS measurements with our results, damage is integrated between the surface and the full amorphizing depth of each studied condition, and normalized by the total damage accumulated in such depth. The simulations shown depths of 38,35 and $35 \mathrm{~nm}$ at which $100 \%$ of the layers are amorphized for RT $10^{19}$, RT $10^{12}$ and $250^{\circ} \mathrm{C} 10^{19}$ $\mathrm{cm}^{-2} \mathrm{~s}^{-1}$ conditions respectively, but identify the mean values of the $\alpha / c$ transitions at 43.6, 39.4 and $39.4 \mathrm{~nm}$. The figure is quite sensitive to such criteria, but the first, smaller, values have been chosen to attain convergence to the measured value of 1 at the end. A value of $35 \mathrm{~nm}$ have been chosen for $250{ }^{\circ} \mathrm{C} 10^{12} \mathrm{~cm}^{-2} \mathrm{~s}^{-1}$ for consistency.

As in the case of Ge ions, the damage cascades used are calculated assuming an amorphized target (a-cascades) for all conditions but for the $250{ }^{\circ} \mathrm{C} 10^{12} \mathrm{~cm}^{-2} \mathrm{~s}^{-1}$ case. This issue, together with the inability of the model to distinguish between RT $10^{12} \mathrm{~cm}^{-2} \mathrm{~s}^{-1}$ and $250{ }^{\circ} \mathrm{C} 10^{19} \mathrm{~cm}^{-2} \mathrm{~s}^{-1}$ conditions, in contradiction with the experimental results is probably pointing out at parameterization problems in our model for temperatures higher than the 
rest of temperatures used in t this study (from $\mathrm{LN}_{2} \mathrm{~T}$ to $\mathrm{RT}$ ), or to problems related to the excess recombination of Ga damage at $10^{12} \mathrm{~cm}^{-2} \mathrm{~s}^{-1}$ dose rates, in contrast with $10^{19}$ $\mathrm{cm}^{-2} \mathrm{~s}^{-1}$ where results seem to be satisfactory.

In the same way as in the case for B implantations at RT (see Fig. 5.13), the calibration for cluster dissolution upon the presented binding energies, was needed to not achieve amorphization in our simulations for the non-amorphizing flux at $250^{\circ} \mathrm{C}$. Disagreement on the beginning of damage accumulation at RT can be explained as a consequence from a not exhaustive parametrization on cluster dissolution depending on their size. Despite this disagreement, the model predicts fairly enough the damage quantity and accumulation regimes up to amorphization for these very different conditions. Unfortunately, in the reported article, no information about the extension of the amorphous layers was given. Thus, these results are not included in Fig. 5.12 .

Finally, we have noticed in our simulations that, while $V$-type cluster dissolution leads to a reduction in the number of these defects but an increase in their size, the case of $I$-type clusters is much different. With the presented model, a reduction in both number and their size is observed. This behavior has already been reported, Boninelli et al. (2012a); Claverie et al. (2010) and the mechanism underlying this non-conservative cluster dissolution is still unknown. We attribute this behavior to the low diffusion rate of Is (see Table 5.1), making the emitted $I$ s not able to travel far from the emitting cluster, while $V$ s are able to either recombine or grow other clusters. These explanations can serve as a starting point for a more specific cluster energetic study, and a hint for this non-conservative behavior.

\subsection{Limitations}

Table 5.2 shows the conditions for which $c$ - or $\alpha$-cascades have been used in this work. As explained in Section 5.3.3, for B ions $c$ - cascades are used until full damage saturation is achieved, where $\alpha$-cascades continue, while for the rest of ions, full $\alpha$-cascades are always used with the exception of $\mathrm{Ga}$ at high temperature $\left(250^{\circ} \mathrm{C}\right)$ and low flux $\left(1 \times 10^{12} \mathrm{~cm}^{-2} \mathrm{~s}^{-1}\right)$ for which the damage calculated with $\alpha$-cascades is way too large. This exception of this extreme case is consistent with the fact that nearly all the damage created by every ion is annihilated dynamically, and the criterion applied fails for this specific case. 
Nevertheless, the need of this fitting parameter used to choose between $c$ - or $\alpha$-cascades presents a strong limitation to the simulator, MMonCa, and the model itself. In fact, by using $c$-cascades for F, Ga and Ge ions at the standard studied conditions, the experimentally measured values of damage quantity and extension of the induced amorphous layer cannot, by far, be reproduced. The same stands for B implantations in which the criterion of establishing the $c \rightarrow \alpha$ transition at the damage saturation point is necessary. The need of a parameter to decide when to use $c$ - or $\alpha$ - cascades would be suppressed by introducing in MMonCa its own BCA simulator, with models such as those existing in Sentaurus Process KMC Sentaurus (2012), in which the pre-existing damage is taken into account.

As it has been mentioned, and although the model works with a high level of agreement with experimental measurements, further research is needed to improve the explicit cases where its predictions fail. These cases are, for instance: the prediction of extended amorphous layers at RT for $35 \mathrm{keV} \mathrm{B}$ implantations at very high fluences (see Fig. 5.13 and description); and the case of $\mathrm{Ga} 30 \mathrm{keV}$ implantations at RT and low flux. To shed light on these issues, further research is suggested, clarifying the validity of the model at higher temperatures at RT, and the possible importance of a better parameterization for cluster dissolution.

\subsection{Conclusions}

To summarize, we have presented a quantitative model for Ge damage accumulation and amorphization caused by ion implantation. The model is based on a examination of the different stages of Ge amorphization that take place within the implantation process.

The main mechanisms analyzed in this work are summarized as follows: i) a threshold value of $\sim 1.3 \times 10^{22} \mathrm{~cm}^{-3}$ damage concentration, for which the $\alpha / c$ transition occurs, and explains different implantation regimes. This value is independent of the implantation conditions. ii) A fitting parameter to decide when to use $c$ - or $\alpha$-cascades. iii) Recombination energies of $A P \mathrm{~s}$ depend on their size. These energies, start at $0.5 \mathrm{eV}$ for the smallest sizes, up to the $2.17 \mathrm{eV}$ for Ge SPER. iv) Dissolution of pure $I$ - and $V$-type clusters, which play an important role for temperatures equal or higher than RT at high fluences. 
This description is able to explain experimental results on damage accumulation and amorphization of Ge for a wide range of conditions. The model has been validated by reproducing reported experiments of B, F, Ga and Ge ions under different temperatures, fluences and fluxes of implantation conditions. Due to the different migration barriers of $I \mathrm{~s}$ and $V \mathrm{~s}$, cluster dissolution result in a different behavior in the evolution of $I$ - and $V$-type clusters.

Conclusions achieved in this study are i) there are different implantation regimes based on the crystallinity and pre-existing damage within the Ge sample; ii) a correct calculation upon the atomistic composition of the generated damage becomes crucial for predicting $\alpha / \mathrm{c}$ interface locations; iii) the diluted implantation-induced damage by light ions is easily annealed dynamically in Ge at relatively low temperatures; iv) induced damage by heavier ions is more compact and consists of a higher number of $I \mathrm{~s}$ and $V \mathrm{~s}$; and v) cluster dissolution becomes important for temperatures equal or higher than RT at high fluences and/or low fluxes.

Further research to account for damage accumulation and de-channeling during the calculation of cascades is also suggested to dynamically calculate the implantation-induced damage. In this line, models such as those existing in Sentaurus Process KMC Sentaurus (2012), in which the pre-existing damage is taken into account, would suppress the need of a parameter to decide when to use $c$ - or $\alpha$ - cascades. Up to date, this is one of the main limitations of our simulator, MMonCa. 


\section{Chapter}

\section{Solid Phase EPITAXIAL REgROWTH IN}

GE

Solid phase epitaxial regrowth (SPER) is a common technique used in the manufacturing processes of MOSFET technology. Even though a relatively broad knowledge is found for $\mathrm{Si}$, there is a greater uncertainty when it comes to Ge, which importance is arising in the last generation of microelectronic devices. To simulate this process, the need of a model which reproduces anisotropic growth and is able to detect and place twin defects becomes relevant, opening the possibility to simulate the interaction of different crystallographies, as it has been observed to be an important factor for some orientations, justifying by this mechanism experimental results.

In this Chapter, a parameterization for Ge of the Lattice Kinetic Monte Carlo (LKMC) model reviewed in Chapter 3 which is able to give an explanation of the different anisotropy effects in the recrystallization of substrate wafers through a defect formation formalism is presented. An agreement between experimental observations and simulations is found by comparing regrowth velocities for different samples at different anneal conditions with LKMC simulations that consider twin defect formation for specific directions. Different regrowth velocities are found for distinct orientations of a SPER process within the annealed sample. The presented calibration is also discussed and compared against other published experimental results found in the literature. Planarization in the first stages of 
the recrystallization process can also be taken into account, to some degree, by considering the initial roughness of the amorphous crystalline interface caused by the amorphization process through ion implantation. A simple calibration to take into account the dependence of SPER in Ge upon the stress caused by the appliance of hydrostatic pressure is also presented. Part of the results presented in this Chapter has been published in Darby et al. (2013) and Gomez-Selles et al. (2014).

\subsection{Introduction}

One of the features well characterized for $\mathrm{Si}$, but not that much modeled in $\mathrm{Ge}$ is the SPER rate dependence on the orientation of the grown substrate. A recent study, Darby et al. (2013) confirms past observations, Csepregi et al. (1977) of strong dependencies on these orientations, mostly due to a strong contribution from twin defect formation during the recrystallization process, which slows down the recrystallization.

Consequently, a parameterization for Ge of the model presented in Chapter 3 has been carried out. It aims to reproduce consistently experimental results for the crucial decanano scales that are the main target of the microelectronic processing industries, where the SPER process is performed within a few nanometers from the surface.

This Chapter is structured as follows: Section 6.2 generally reviews the model presented in Chapter 3, dealing with both planar orientation dependence on local configurations, and twin defect formation. Section 6.3 reproduces actual experiments, Darby et al. (2013) to validate the model when compared with the measured values of SPER rates and visual observations. In Section 6.4, the presented calibration is compared with other reported results 6.4.1), insights for the planarization process at the first stages of SPER in Ge are given $(6.4 .2)$ and validity of previous model which takes into account the influence of hydrostatic pressure in Ge SPER is discussed through a simple calibration of the model already presented for $\mathrm{Si}$ in Chapter 3 which fits a good match with reported observations at different temperatures for SPER in Ge 6.4.3. Finally, in Section 6.6 the main results achieved in this Chapter are summarized, and further research in this field are suggested. 


\subsection{LKMC modeling}

A Lattice Kinetic Monte Carlo (LKMC) model which is able to obtain distinct recrystallization rates for different planar orientations taking into account facet formation during SPER by simulating the crystalline lattice is proposed.

As explained in Chapter 3, different SPER velocities are found by carrying out simulations consisting on a full template of crystalline Ge lattice against an amorphous (untemplated) Ge phase. The very next amorphous Ge layer to the $\alpha / \mathrm{c}$ interface is progressively crystallized when the number of atoms with two undistorted bonds, Drosd and Washburn (1982) is the one needed by the planar orientation. These numbers are 1, 2 and 3 for $\{100\}$, $\{011\}$, and $\{111\}$ orientations, respectively.

Processes are modeled through three different Arrhenius rates with an equal activation energy, and a different prefactor for each one of them. A change in the velocities in the orientations is achieved through these three planar lattice constants, $K(100), K(011)$ and $\mathrm{K}(111)$, expressed in atoms/s as:

$$
\nu=K(\text { site }) \exp \left(\frac{-E_{\text {activation }}}{k_{B} T}\right) .
$$

As it has been observed in previous works, Martin-Bragado and Moroz (2011) it is important to consider a difference in those prefactors depending on the neighbor coordination number encountered at the time of bonding into the lattice, Williams and Elliman (1983). This is modeled by introducing a lower rate in the $\{100\}$ microscopic configurations when less first neighbors are found (6 and 7 in these simulations) making the bond less probable.

Twin defects have also been considered, as described in Chapter 3, modeled through a $50 \%$ probability of forming a twin when a $\{111\}$ configuration is regrown.

\subsection{SPER orientation dependence}

Darby et al. (2013) studied the SPER orientation dependence of a set of Ge wafers polished to various orientations. These substrates were implanted with $1 \mathrm{MeV}, 1 \times 10^{15}$ $\mathrm{Ge} / \mathrm{cm}^{-2}$ ions, leading to a continuous amorphous layer of $\sim 800 \mathrm{~nm}$ for all the samples. Then, these samples were annealed at $330^{\circ} \mathrm{C}$, inducing recrystallization in the substrates, 
and the velocities of recrystallization were compared for angles of $0^{\circ}$ (which corresponds to a (001)Ge substrate) $15^{\circ}, 25^{\circ}, 40^{\circ}, 54.7^{\circ}$ (i.e. (111)Ge) $70^{\circ}, 80^{\circ}$ and for a (011) Ge substrate, equivalent to $90^{\circ}$ away from the (001)Ge. This study resulted in a strong velocity dependence on the substrate orientation, with the [001] direction showing a velocity 16 times faster than the [111] direction. SPER of (111) Ge was the only orientation which showed twin defect formation, but in a less quantity than that reported for Si, Rechtin et al. (1978).

\subsubsection{Simulations}

As it has already been mentioned, the experiments described above were performed by producing $800 \mathrm{~nm}$ width amorphous layers. For our simulator, these dimensions are highly expensive in terms of RAM memory consumption. Since the simulations were performed to help understanding the SPER process, and the interest relies on the first nm of planar $\alpha / \mathrm{c}$ interface recrystallization, the interface was simulated by defining a mesh with a maximum depth of $160 \mathrm{~nm}$ in the regrowth direction, instead of simulating the whole $1 \mathrm{MeV} 1 \times 10^{15}$ $\mathrm{Ge} / \mathrm{cm}^{-2}$ implantation followed by the SPER process. The interface was automatically defined after prefixing $\mathrm{c}$-Ge and $\alpha$-Ge phases at the beginning of the simulations and at the desired depth.

Using an activation energy of $2.17 \mathrm{eV}$ and the prefactors listed in Table 6.1 the simulations described below have been performed in order to reproduce the results showed in Ref. Darby et al. (2013).

\begin{tabular}{lr}
\hline \hline Configuration & Prefactor(atoms $/ \mathrm{s})$ \\
\hline $\mathrm{K}\left(100_{h}\right)$ & $2.35 \times 10^{18}$ \\
$\mathrm{~K}\left(100_{l}\right)$ & $1.18 \times 10^{17}$ \\
$\mathrm{~K}(011)$ & $2.41 \times 10^{16}$ \\
$\mathrm{~K}(111)$ & $1.50 \times 10^{12}$
\end{tabular}

Table 6.1: Recrystallization prefactors for the local configurations. $h$ and $l$ stand for high and low recrystallization rate, depending on the neighbor coordination number. 
Figure 6.1 presents a comparison between the measured data versus our model for Ge SPER annealed at $330^{\circ} \mathrm{C}$, grown at [100], [111], and [011] directions, with corresponding angles of $0^{\circ}, 54.7^{\circ}$ and $90^{\circ}$ respectively.

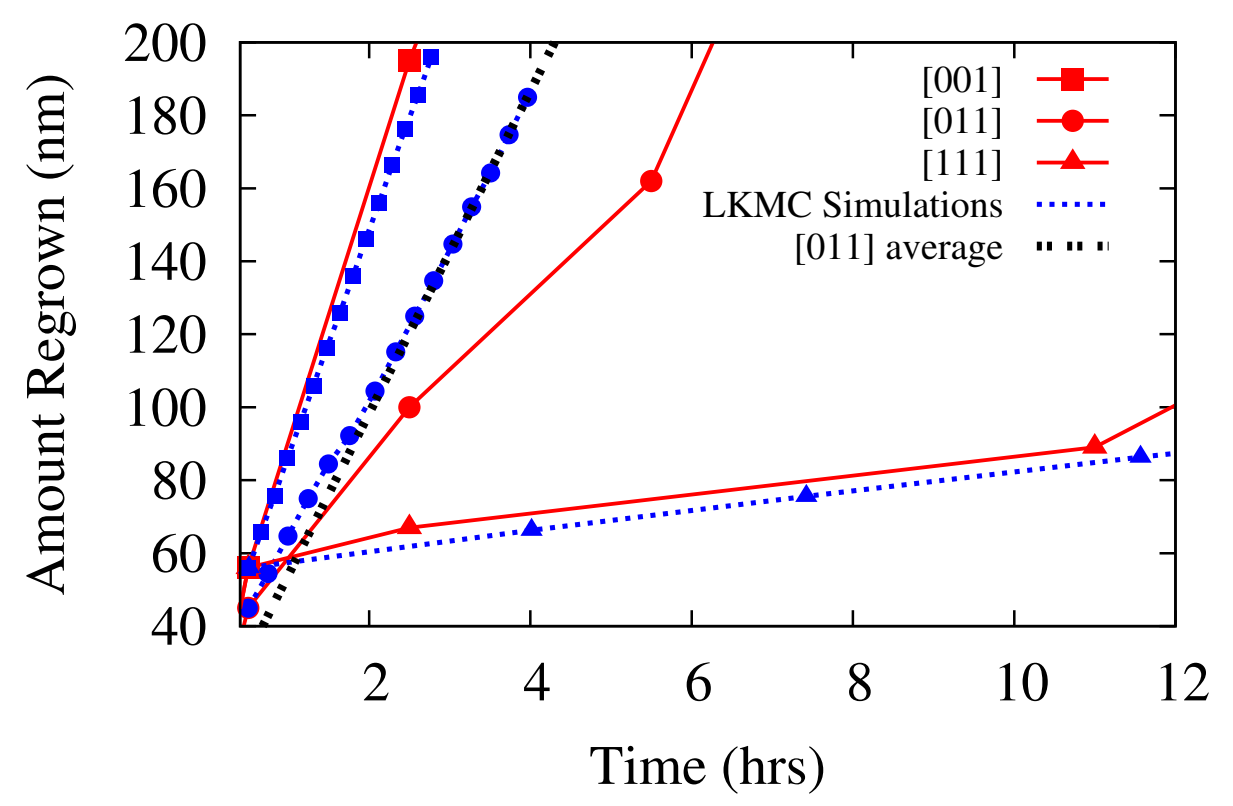

Figure 6.1: Comparison between experimental results Darby et al. (2013) (solid lines) and LKMC simulations (dashed lines) for the three main regrowth directions: [001], [011] and [111]. The first $200 \mathrm{~nm}$ of Ge SPER at $330^{\circ} \mathrm{C}$ are shown. A linear regression in the [011] configuration has been done to fit the simulation of that orientation.

The experimental first 30 minutes ( $\sim 50 \mathrm{~nm}$, depending on the configuration) measured correspond to a planarization process which is not taken into consideration for the results Darby et al. (2013). Such process could be simulated for other less computationally expensive conditions, and will be more deeply discussed in Section 6.4.2. The calibrated total rates refer to a linear analysis for the whole depth, presented for the (011)Ge substrate in the graph to show the fitting performed on the parameters to the average slope. As it has already been pointed out, the average value for thick samples might not be as useful in the microelectronic industry as the values obtained for thin SPER. In our simulation setup, the cell has a cuboid geometry with a rectangular base of around $50 \mathrm{~nm}$ edge per axis in the YZ plane, depending on the planar configuration due to issues of periodicity in the lattice. 
a) $0^{\circ}$

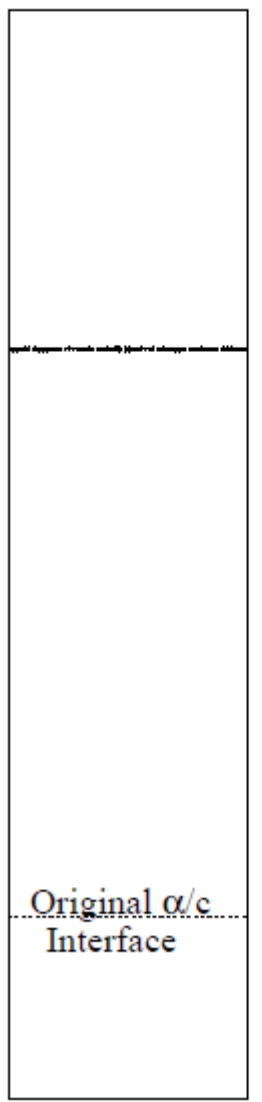

b) $54.7^{\circ}$

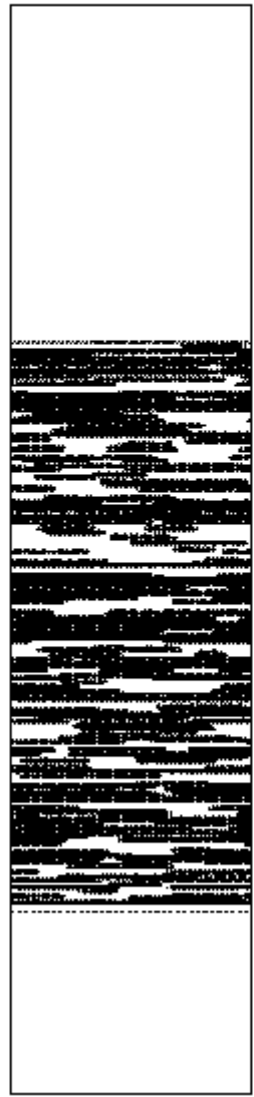

c) $90^{\circ}$

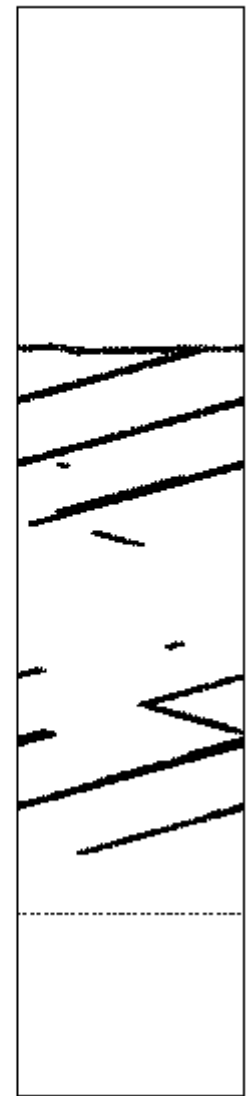

Figure 6.2: Final atomistic configurations of the $150 \mathrm{~nm}$ of simulated SPER corresponding to the results of Figure 6.1. Only Ge $\alpha / \mathrm{c}$ interface and defective-positioned atoms (twins) are plotted.

Then, the SPER process was performed through the X axis up to $150 \mathrm{~nm}$. Slight changes in the slope of the experimental results, and differences reported in the literature might be explained as in Claverie et al. (2010): small variations in temperature when performing the experimental procedure have big impacts. We will come back to discuss this issue, by comparing with other reported experiments in Section 6.4.1. An example of the results reported is presented in Figure 6.2, where a 2D representation of the final regrowth of this process is shown. It can be appreciated that, as expected, the (111)Ge substrate $\left(54.7^{\circ}\right)$ is more defective than the others. 


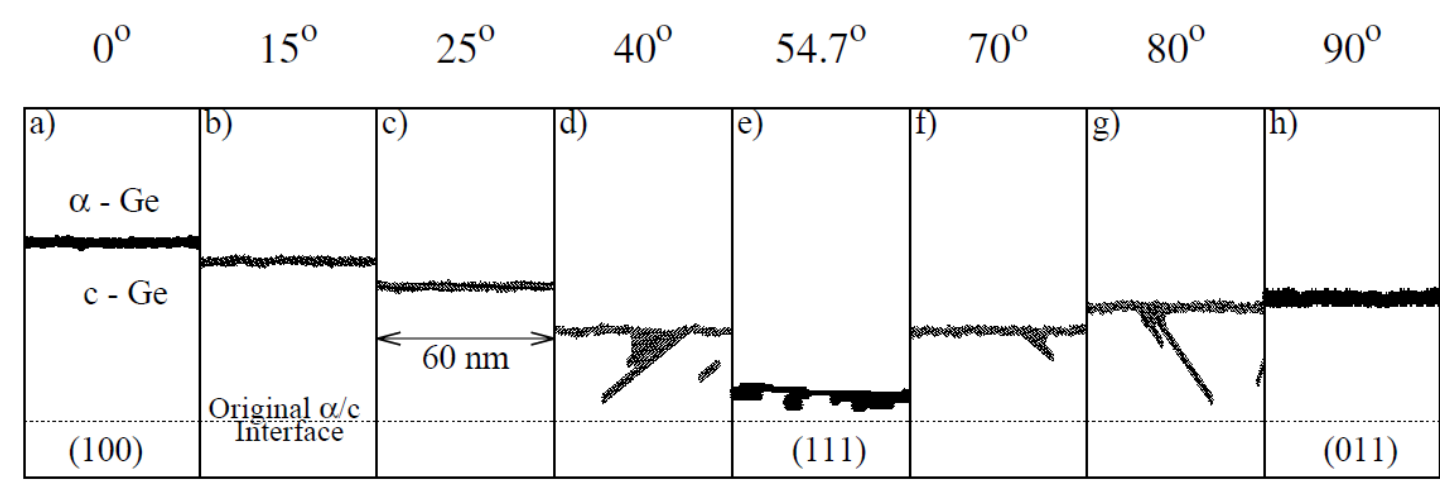

Figure 6.3: Ge $\alpha / \mathrm{c}$ interface and defective-positioned atoms (twins) of an isochronal anneal for eight different configurations at $330^{\circ} \mathrm{C}$ and $1000 \mathrm{~s}$. a) corresponds to the fastest direction, (100) e) to the slowest, (111) and $\mathrm{h}$ ) is (011). The orientations are a) $0^{\circ}, \mathrm{b}$ ) $15^{\circ}$, c) $25^{\circ}$, d) $40^{\circ}$, e) $54.7^{\circ}$, f) $70^{\circ}$, g) $80^{\circ}$, h) $90^{\circ}$.

Figure 6.3 is an atomistic representation of a $1000 \mathrm{~s}$ isochronal annealing process at $330^{\circ} \mathrm{C}$ for a wide range of orientations. Position of the $\mathrm{Ge} \alpha / \mathrm{c}$ interface and defectivepositioned atoms of Ge are shown. The low defect roughness for the (100)Ge substrate corresponds to the fastest growing SPER (a), while the rough (111)Ge substrate corresponds to the slowest orientation. An intermediate roughness between $\mathrm{Ge}(111)$ and $\mathrm{Ge}(100)$ is also observed for $\mathrm{Ge}(011)$.

Figure 6.4 shows a comparison between experimental and simulated data with the same substrate orientations as in Fig. 6.3. In the experimental data, a total rate through linear analysis after the first 30 minutes of planarization has been held, proceeding for all orientations in the same way as before. Those planar directions have been simulated with a cell geometry of $180 \times 20 \mathrm{~nm}^{2}$ with a SPER amount of $21 \mathrm{~nm}$ in the regrowth direction to be recrystallized.

\subsubsection{Discussions}

Although the overall match between experiments and simulations in Figs. 6.1 and 6.4 is good, the partial disagreement found for the [011] direction deserves further explanation. On the one hand Csepregi et al. (1977) reported a lower velocity for $\mathrm{Ge}(011)$ than the one 


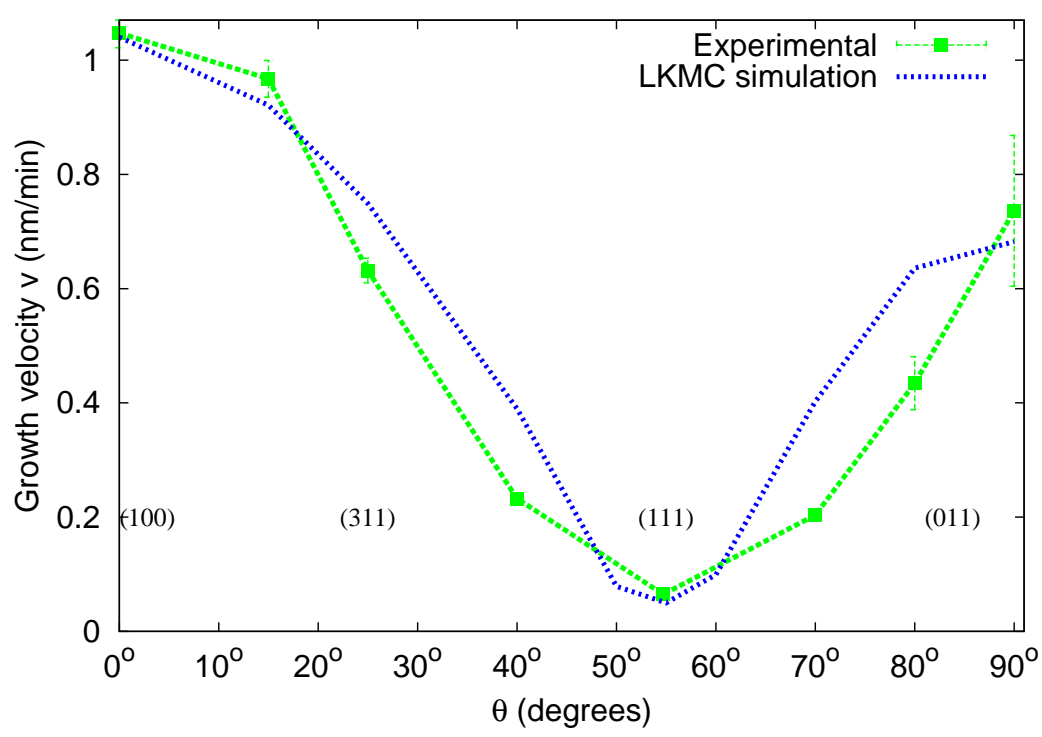

Figure 6.4: Comparison of experimental Darby et al. 2013) Ge SPER velocities (solid line) and LKMC Simulations (dashed line) for a range of orientations between $0^{\circ}$ and $90^{\circ}$ at $330^{\circ} \mathrm{C}$.

achieved with the used calibration. On the other hand, the measured values for recrystallization in the [011] direction represented in Fig. 6.1 seem to present an initial low velocity from 40 to $160 \mathrm{~nm}$, speeding up after that. Because of that, and as explained in Section 6.3.1, the parameterization carried out refers to the average value shown in that figure. Does this reflect the true nature of Ge SPER? Some extra research would be needed to clarify SPER process on the first nm of regrowth. Other deviations between experimental and simulation results may be a consequence of a mechanism reported by Duffy et al. (2011) that we have not seen modeled yet: a process is involved after twin defects are formed in the first seconds of anneal that heals such damage, leaving the structure less defective. This would have an impact in the velocities for directions near the [111], but again, more research would be needed to corroborate such hypothesis. Still, a compromise in the parameterization of the model between the results of Figure 6.1 for high amounts of material regrowth and for a greater number of orientations (Figure 6.4 has been made.

It becomes clear in Figure 6.2 that local configurations of $\{111\}$ are the most important source of defects in Ge, as it is in Si, Martin-Bragado (2011), lowering the velocities of SPER. Further research in that process might elucidate also the differences in the wide 
range of orientations, due to the relationship found between the existence of defects and growth velocities.

It is also relevant that a bi-modal growth is not found for the $\{111\}$ configuration in Ge, as it has been demonstrated for Si Csepregi et al. (1978); Martin-Bragado and Sklenard (2012). Considering the explanation given in Chapter 3, where an statistical mechanism in which two $\{111\}$ twin planes engage into $\{011\}$ microscopic configurations of recrystallization, this can be explained through the fact that (111)Ge regrown substrates present a less concentration of twin defects, Darby et al. (2013) when compared to higher Si concentrations, Rechtin et al. (1978) thus limiting this mechanism to occur.

\subsection{Other considerations}

\subsubsection{Temperature calibration}

The presented model has been validated against the experimental results published by Darby et al. (2013). Nevertheless, the model should also work for other measurements reported in the literature. As it has already been pointed out, Claverie et al. (2010) showed that the large discrepancies in the recrystallization velocities between different reported experiments in the literature can be explained through small variations in the temperature due to the difficulties existing to control and measure the temperature of the surface of a Ge wafer at processing temperatures. These discrepancies were explained through reasonable maximum systematic errors in the temperature measurements of $16 \mathrm{~K}$.

In order to check the model and calibration presented in Section 6.3.1 against other works, simulations have been done in the same way as in Figs. 6.3 and 6.4, but for different temperatures and with only (001)Ge substrates.

Fig. 6.5 is a comparison between experimental, Csepregi et al. (1977); Lu et al. (1990) (symbols), our LKMC simulations (symbols plus lines) and the fit performed in Claverie et al. (2010) following the Arrhenius law:

$$
\nu=3.5 \times 10^{16} \exp \left(-\frac{2.16}{k_{B} T}\right) \mathrm{nm} / \mathrm{s}
$$

The difference between the mentioned fit and our results is in the range of $\sim 5 \mathrm{~K}$. In 
other words, with the presented calibration, the results reported in the literature can be reproduced by slightly varying the simulation temperatures.

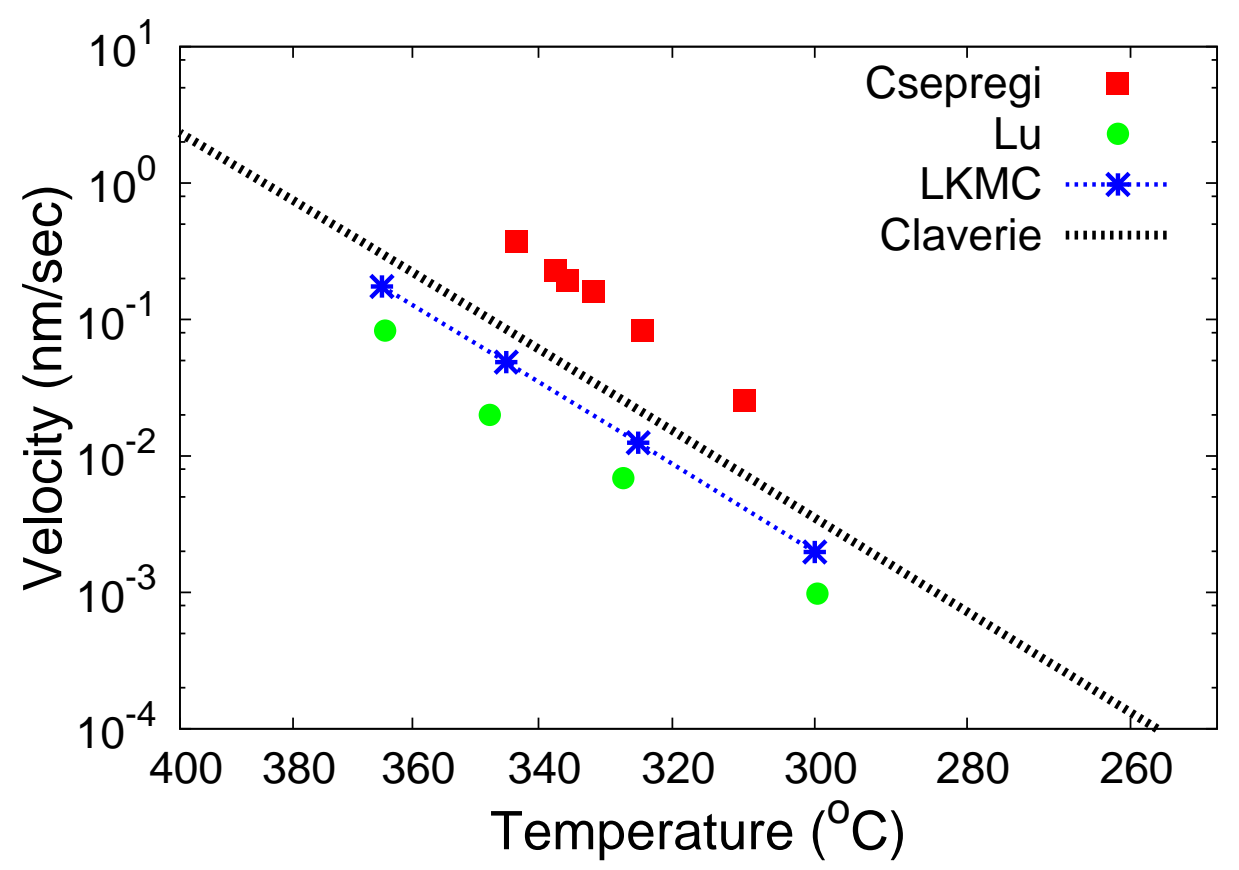

Figure 6.5: Comparison between simulations using the calibration presented in Darby et al. (2013) and experimental results Csepregi et al. (1977); Lu et al. (1990) along with the fitted Arrhenius law of Claverie et al. (2010) for Ge SPER velocity as a function of the process temperatures.

Fig. 6.6 is a comparison between experimental results, Claverie et al. (2010) and KMC simulations of the $\alpha / \mathrm{c}$ interface evolution over time at $310{ }^{\circ} \mathrm{C}$. The first point corresponds to the as-implanted value after a $150 \mathrm{keV}, 5 \times 10^{14} \mathrm{Ge} / \mathrm{cm}^{-2}$ ion implantation. The rest of the data shows the $\alpha / \mathrm{c}$ interface progress after different annealing times. Points were extracted through image analysis from the published XTEM images.

The differences shown in Fig. 6.5 between our calibration and other reported experimental data can now be interpreted, as well as the influence in the recrystallization velocities induced by slight variations in the Ge surface temperature during the SPER process. While simulations performed at $310{ }^{\circ} \mathrm{C}$ do not follow a good agreement, by increasing 5 ${ }^{\circ} \mathrm{C}$ in the simulations a good match is achieved. 


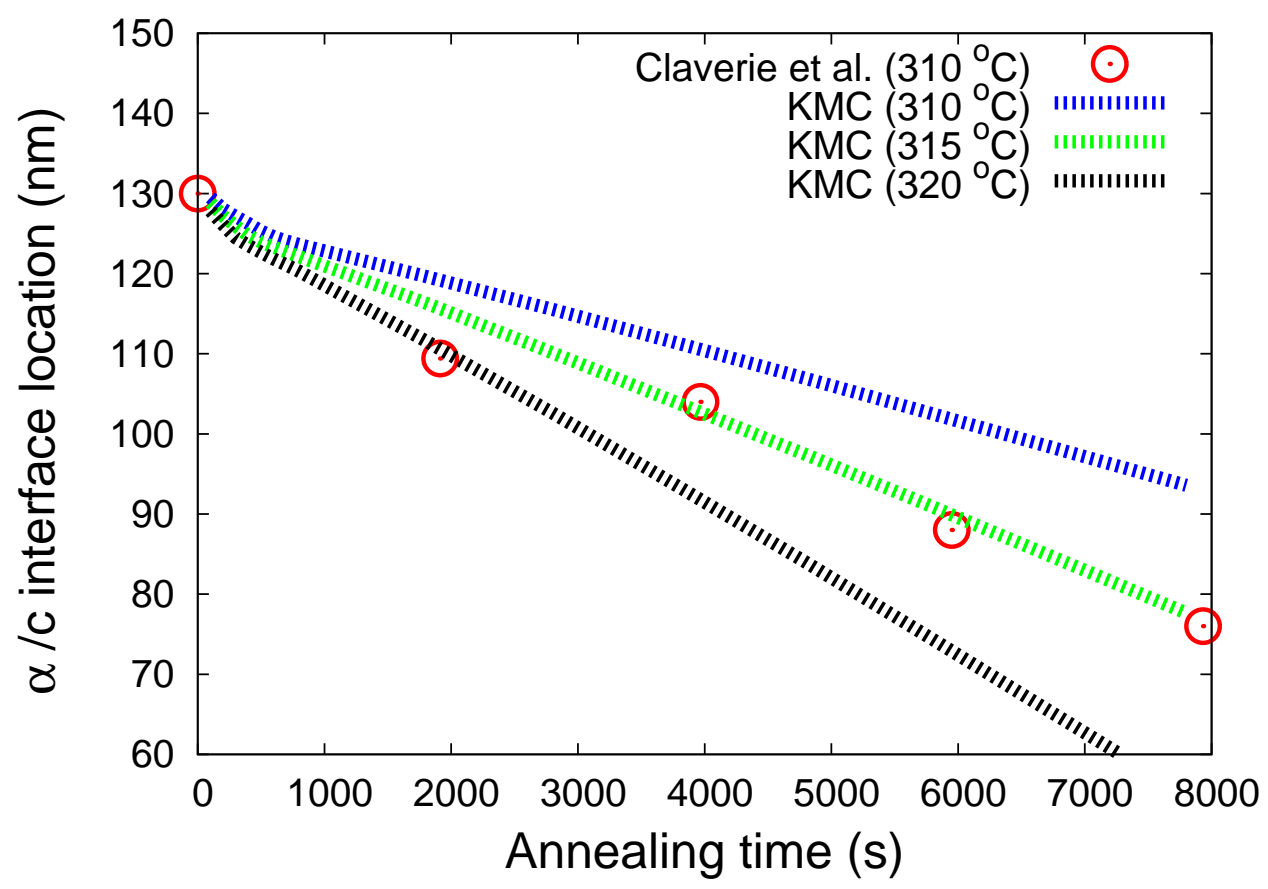

Figure 6.6: Comparison between experimental, Claverie et al. (2010) (symbols), and KMC simulations (lines) of the $\alpha / \mathrm{c}$ interface position for increasing annealing times at $310^{\circ} \mathrm{C}$ after a $150 \mathrm{keV}, 5 \times 10^{14} \mathrm{Ge} / \mathrm{cm}^{-2}$ implant. Using the calibration presented in Darby et al. (2013) a shift of $5{ }^{\circ} \mathrm{C}$ is needed to achieve a good match.

\subsubsection{Planarization}

In Fig. 6.1, the first 30 minutes of SPER were not taken into account in order to calibrate the SPER of planar $\alpha / c$ interfaces, because they presented a different, faster velocity due to a planarization process. By simulating the SPER process of Ge starting from a perfect planar $\alpha / c$ interface, this process cannot be reproduced, since the initial roughness is not taken into account. Nevertheless, by simulating the whole implantation (described in Chapter 5 plus SPER (described above) processes in Ge, these issues might be assessed.

In our simulations, two different trends are displayed in Fig. 6.6 for the first nm of recrystallization. This trend is similar to those reported by Darby et al. (2013), for which a faster SPER velocity was found for all orientations (see Fig. 6.1). This planarization can be simulated by using both OKMC, for amorphization, and LKMC, for SPER, models: 
the interface roughness appears as a consequence of the amorphization process, while it becomes more planar for increasing annealing times. Fig. 6.8 is a set atomistic representations, extracted from the simulations represented in Fig. 6.6, for the planarization process at the first stages of SPER. The atoms belonging to the $\alpha / \mathrm{c}$ interfaces are inserted by the LKMC module, while defects in the c-phase are from the OKMC module. In the top inset (as-imp) a very rough interface is produced from the ion implantation. As the sample is subsequently annealed for increasing times, the amorphous phase recrystallizes from the crystalline seeds until it forms a roughless, planar $\alpha / \mathrm{c}$ interface. This process results in a higher velocity for SPER: the local interfaces which are deeper into the substrate, heal the roughness, while those in touch with the pure $\alpha$-Ge phase, advance the recrystallization, resulting in a faster regrowth, until a planar interface is formed, and the velocity stabilizes to a constant SPER rate.

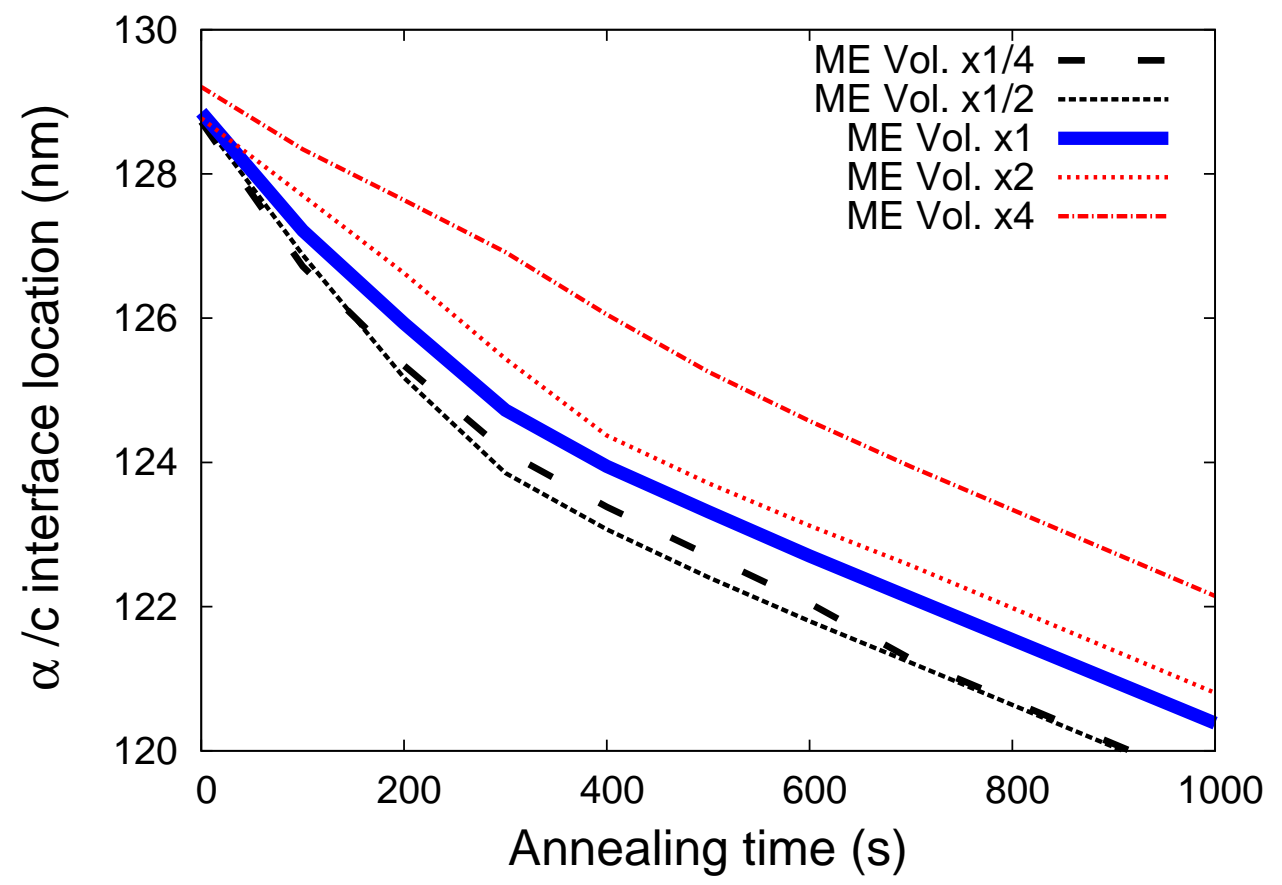

Figure 6.7: Comparison for different volumes of ME for the planarization process at the first $1000 \mathrm{~s}$ of anneal in Fig. 6.6. Volumes are referred to the typical ME volume size of $1.5 \mathrm{~nm}^{3}$. 


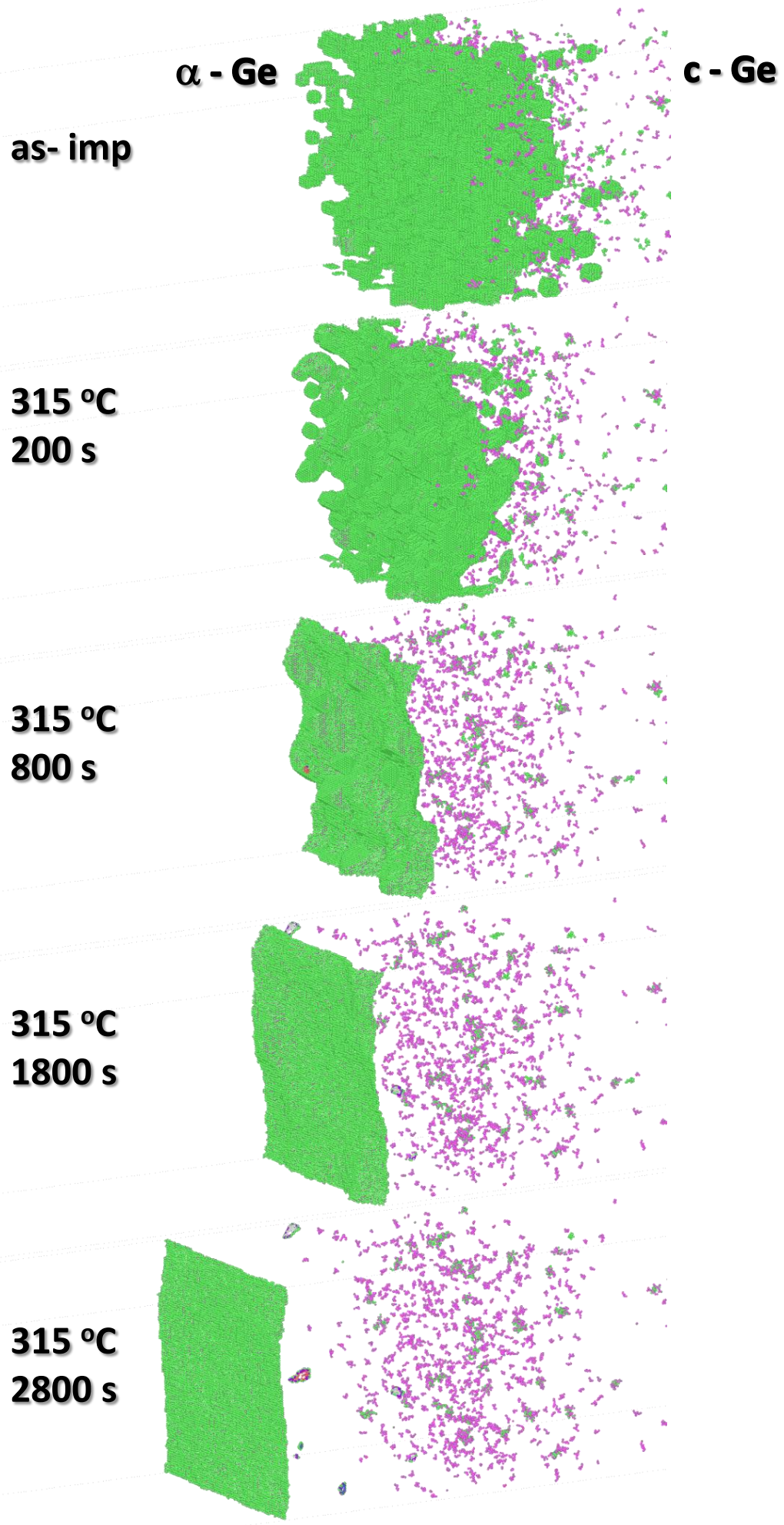

Figure 6.8: Atomistic view of a planarization process. Represented with OVITO, Stukowski (2010) 
Fig. 6.7 is a comparison of the simulation results obtained by using different volumes of ME for the planarization process at the first 1000s of anneal shown in Fig. 6.6. Volumes are referred to the typical ME volume size of $1.5 \mathrm{~nm}^{3}$ used in MMonCa by default. It is noteworthy that by using reasonable values of volume, the results are very similar, while when using very high ME volumes, i.e. 4x, the information of the roughness is lost, and the process cannot be simulated. Even though the trends seem similar to those experimentally observed in the mentioned cases, a more systematic study on this process is needed and suggested to be done in the future. By doing so, it could be assessed if this behavior is a result of an artifact in the code, or if some extra mechanisms should be added to the models, such as stress dependence on the quantity of damage or $\alpha / c$ interface roughness, as suggested by Darby et al. (2011).

\subsubsection{Stress-induced effects in Ge SPER: Hydrostatic Pressure}

Due to the already mentioned reasons on why Ge has not been yet studied as Si in many situations, stress effects on Ge SPER lack of study, at least, with the same emphasis as reported for Si. Nevertheless, as it has been also pointed out, due to the similarities between both Si and Ge materials, usually the same Si models can be applied into Ge modeling with small modifications. Following this line, a preliminary study upon the effects of hydrostatic pressure on Ge SPER has been done. Due to this lack of bibliographic information upon the mentioned effects, it has only been possible to perform a rough adjustment of the parameters calibrated to $\mathrm{Si}$, already presented in shown Chapter 3.

Lu et al. (1990) studied the influence caused by the appliance of hydrostatic pressure in the SPER of Ge. An enhancement of the SPER rate greater than a factor 100 over that at ambient pressure was found due to hydrostatic pressures of up to $5.2 \mathrm{GPa}$. It was characterized by a reduction in the negative activation volume of $\sim 45 \%$, considering that an enhanced velocity of SPER under compressive stress is due to a negative activation volume. Lu et al. (1991) also reported a negative activation volume for Si of $\sim 28 \%$ which is $\sim 60 \%$ lower than the value found for Ge. By applying a simple proportional adjustment in the activation strain tensors reported by Sklenard et al. (2014) for Si (see Table 3.2) we have obtained the calibrated parameters presented in Table 6.2.

With this very simple approach, hydrostatic pressure appliance effects upon SPER in 


\begin{tabular}{ccc}
\hline \hline $\mathrm{c} 1 \mathrm{NN}$ & $\mathrm{V}_{\|}\left(\Omega_{G e}\right)$ & $\mathrm{V}_{\perp}\left(\Omega_{G e}\right)$ \\
\hline 2 & -0.57 & 0 \\
3 & -45 & 23
\end{tabular}

Table 6.2: Values of the components of Ge activation strain tensors depending on the number of crystalline first neighbors $(\mathrm{c} 1 \mathrm{NN}) . \Omega_{G e}$ is the molar volume of Ge.

Ge can be reproduced, showing the validity of the model in Ge too, as can be seen in Figure 6.9. Nevertheless, further research should be conducted. By performing experiments on the effects produced by both in-plane and normal uniaxial stress in Ge SPER, this preliminary parameterization could be validated or refuted, and the main limiting or precursor events elucidated.

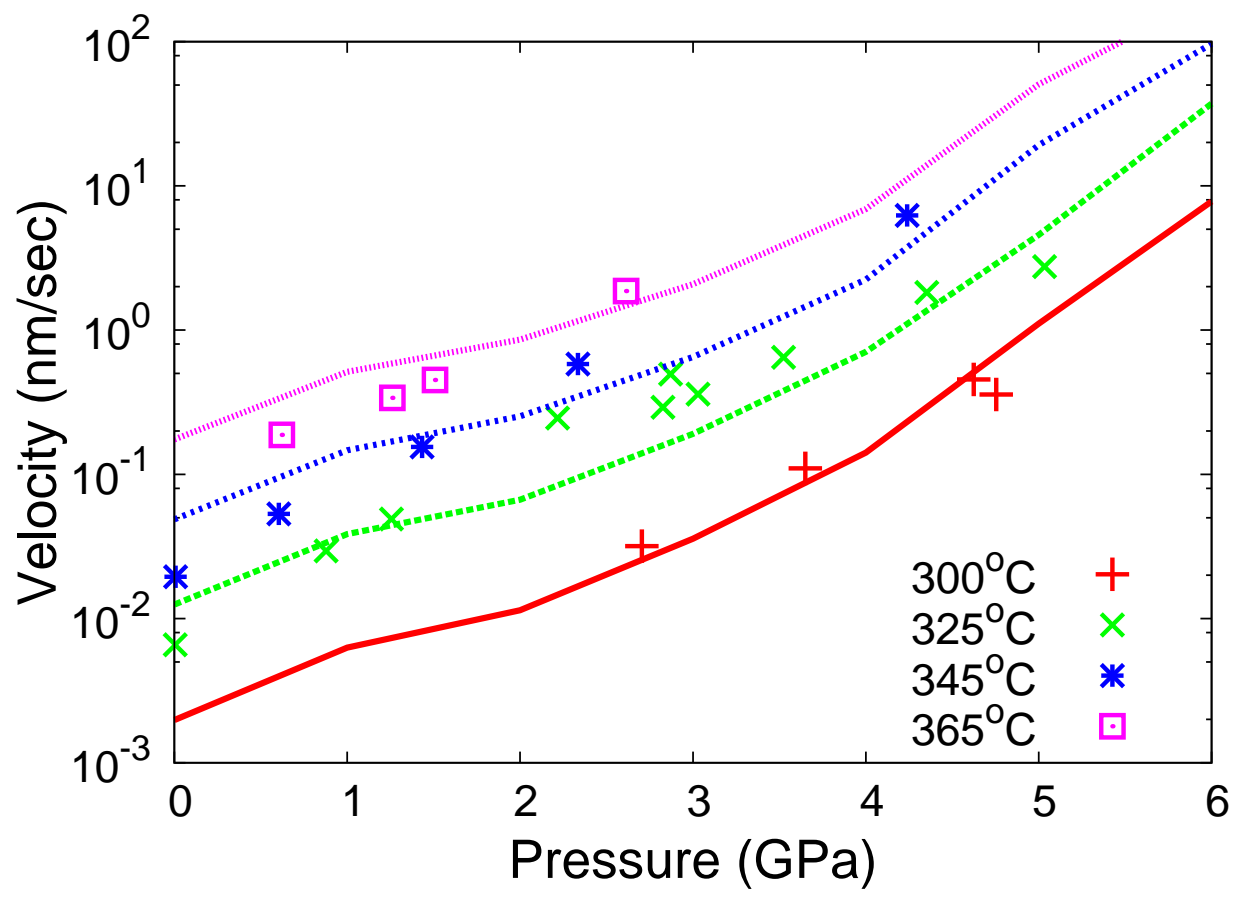

Figure 6.9: Comparison between simulations (lines) and experimental results (symbols) Lu et al. (1990) of Ge SPER velocity as a function of the pressure for different temperatures. 


\subsection{Limitations}

- Further mechanisms might be needed to improve Ge SPER dependency with substrate orientations.

- As shown in Fig. 6.7, amorphization + SPER models are mesh sensitive, and care should be taken choosing the mesh elements volumes.

\subsection{Conclusions}

Different epitaxial growth velocities for each substrate orientation reported for Ge have been modeled successfully using a LKMC algorithm. Twin defects formed for $\{111\}$ recrystallization events are the main source of defects in Ge SPER, while not bimodal SPER is found for Ge. This is proposed to be a consequence of the lower concentration of twin defects in $\mathrm{Ge}$, when compared to $\mathrm{Si}$, for which bimodal growth is found. Simulations match with the experimental results reasonably well for (100) and (111) orientations, while a partial disagreement in the (011) direction is found. This might be due to small variations in the Ge surface during SPER. The proposed model and calibration works for different reported data in the literature and the work presented supports the theory that the differences in SPER rates of Ge between authors may be due to these small variations.

By coupling both OKMC and LKMC for full simulations of amorphization by means of ion implantation followed by SPER, the SPER velocity results in a faster regime of recrystallization of the first few nanometers until a planar $\alpha / \mathrm{c}$ interface is formed. Further research in this issue is suggested.

A simple calibration, based on applying the same ratio between reported activation volumes for $\mathrm{Si}$ and Ge into the activation strain tensors for previous Si models of stress dependent SPER. This has allowed us to achieve a good match between our simulations and reported experimental results for SPER velocities at different temperatures under the appliance of hydrostatic pressure.

Further modelization work on the reported mechanisms of twin defects produced after SPER of (111)Ge substrates is suggested, as well as experiments on the influence of both in-plane and uniaxial stress may cause in Ge SPER. This would help to better understand 
Ge SPER, and improve the presented models. 


\section{CONCLUDing COMMENTS}

In this work, damage accumulation and amorphization caused by ion implantation, as well as Solid Phase Epitaxial Recrystallization of Ge have been studied through physically based kinetic Monte Carlo (KMC) simulations, being able to reproduce realistic scale material processing stages for the semiconductor industry. The main achieved results and conclusions during this work are summarized.

\subsection{Damage accumulation and amorphization in Ge by means of ion implantation}

The different stages of Ge amorphization that take place within the ion implantation process have been studied. A threshold value, in terms of the induced damage, which is independent of the implantation conditions has been provided, being $\sim 1.3 \times 10^{22} \mathrm{~cm}^{-3}$ damage concentration. When the damage locally overcomes this barrier, the $\alpha / c$ transition occurs. This description has been able to explain different damage accumulation regimes. It has been depicted how damage is first slowly accumulated, formed by agglomeration of $I \mathrm{~s}$ and $V \mathrm{~s}$ in the form of $A P \mathrm{~s}$. Then, a dramatic increase in the accumulated damage is explained through collapsing areas, from the crystalline to the amorphous phase. For higher doses, a point in which full extended amorphous layers are finally formed is achieved, and the quantity of damage saturates. In this last regime, the damage production only 
contributes to widen those layers.

The damage caused by $\mathrm{B}$ ions is configured by diluted, small-sized $A P$ s when compared with the compact and bigger APs caused by Ge ions. Dynamic annealing occurring while implanting the ions is mainly modeled through size-dependent recombination energies of the formed $A P \mathrm{~s}$ in the range $0.5-2.17 \mathrm{eV}$. With this description, the reported existing differences in damage accumulation and amorphization caused by different ions are well accounted for. The model has been validated by successfully reproducing reported experimental results of $\alpha / \mathrm{c}$ interfaces caused by different ions at different fluences, fluxes and temperatures. For high temperatures and/or low fluxes, dynamic annealing results in pure $I$ - and $V$-type cluster dissolution.

\subsection{Solid Phase Epitaxial Regrowth of Ge}

Orientation dependence of Ge SPER has been modeled through an atomistic LKMC approach. The strong differences between the experimentally found recrystallization velocities for (001)Ge, (011)Ge and (111)Ge grown substrates are explained as a consequence of the different atomistic environment surrounding the recrystallizing atoms. These are modeled with different growth rates depending on the number of crystalline neighbors and the orientation of each new atom that is attached to its lattice position in a thermally activated mechanism. The activation energy of $2.17 \mathrm{eV}$ for planar $\alpha / c$ interface SPER is confirmed and prefactors for the Arrhenius expressions depending on the orientation are provided. No bi-modal (111)Ge growth is found due to the lower concentration of twin defects formed for $\{111\}$ recrystallization events when compared with Si. Nevertheless, these twins are also proposed as the main source of defects in Ge SPER. This description allows to find very good agreements between simulations and reported experimental results for the velocities of recrystallization for different orientations, from $0^{\circ}$ to $90^{\circ}$ away from the (001)Ge.

The presented study supports the hypothesis of strong temperature dependence of the recrystallization velocities. Thus, by slightly varying the simulated temperatures, good agreement can be also found with experimental results reported by different authors.

Full simulations of amorphization by means of ion implantation followed by SPER 
allows to simulate a planarization processes, existing in the first recrystallization stages. In this process, the rough implantation-induced interface reduces it width, resulting in a faster regime of recrystallization of the first few nanometers until a planar interface is formed. This is done by coupling both OKMC and LKMC models.

Finally, the same ratio between reported activation volumes for $\mathrm{Si}$ and Ge into the activation strain tensors for previous Si models of stress dependent SPER have been applied. This has allowed to achieve a good match between simulations and reported experimental results for SPER velocities at different temperatures under the appliance of hydrostatic pressure. 
The work presented in this dissertation may well serve as a starting point for future works in the field of atomistic simulations for Ge front-end processing. In this concluding lines, investigations that may be done as a continuation of this work on amorphization and SPER in Ge are introduced. Even though it has not explicitly been discussed, existing models and parameterizations for amorphization and SPER in Si and those presented in this work for Ge may also be used to understand those processes for SiGe alloys.

\subsection{Amorphization}

The amorphization model presented in this work has been validated for different ions, spanning for different masses: from the light B up to Ge. Nevertheless, there is still room for validations in the subject. One interesting feature to be checked is the behavior of noble gases, and their impact in amorphization when compared to other similar ions. In Si, Mok et al. (2008) observed that a pre-amorphized volume surrounding the implanted ion was needed to be included in the simulations in order to achieve results consistent with the experiments. Simulations done with the presented models compared against the extensive results reported by Decoster and Vantomme 2009) would be clarifying on whether this behavior is similar in Ge or not.

One of the main limitations of MMonCa is the lack of an integrated and functional 
BCA simulator. This limitation obliges the user to utilize other tools such as SRIM or Sentaurus Process, with the post- and pre-processing effort that it entails. As it has been explained thorough this work, damage cascades are introduced one by one into MMonCa. This involves differentiating between "crystalline" or "amorphous" conditions of implantation, leading to differentiations between ions. This is notorious in the fact that while only cascades computed at the amorphous regime can be used for Ge implants, mixed amorphous-after-crystalline cascades have to be used for B ions. This issue would easily be solved by coupling BCA calculations and KMC simulations dynamically, being able to distinguish pre-amorphized areas from those lacking of defects on-the-fly.

\subsection{SPER}

Regarding the LKMC modelization for Solid Phase Epitaxial Regrowth in Ge, opened lines have been provided. The partial disagreements between experimental results and the simulations presented in this work for orientations different to those of the main (001)Ge, (011)Ge and (111)Ge substrates can be investigated. The mechanism described by Duffy et al. (2011) as "The curious case of thin-body Ge crystallization" in which the defects caused by SPER are healed at relatively low processing temperatures, may be included in the simulations. This may play a role in the velocities of recrystallization, and might be able to explain the mentioned slight discrepancies.

Another main milestone to be achieved in the future would be to perform experiments and simulations to understand the influence of SPER under in-plane or normal uniaxial stress conditions. It would help to elucidate if the mechanisms underlying such processes are similar to those occurring in $\mathrm{Si}$ or not, as well as validating or refuting the presented simple calibration in terms of extrapolating the ratios between activation volumes.

\subsection{End of Range defects in Ge}

After amorphization of the sample and subsequent SPER, a well-defined layer of defects is normally formed behind the original $\alpha / \mathrm{c}$ interface. These defects are commonly referred as end-of-range, or EOR defects. Formation and behavior of these defects under annealing 
have been partially studied in the recent years, but there are several topics that should be further studied. The provided models in this work open the possibility to clarify some of these aspects. Panciera et al. (2010) were able to extract the concentration of $I$ s per dislocation loops. These are supposed to be the main type of EOR defects in Ge, opposed to the $\{311\}$ s that easily appear in Si. Boninelli et al. (2012a) also studied the evolution of EORs as a function of their distance to the surface by amorphizing the Ge sample with selfimplantation at two different energies. By using this reported information together with the presented models in this work, simulations can be carried out in order to understand the mechanisms of formation and dissolution of this type of defects. Some of the results that these techniques would allow to obtain comprises the influence of vacancy injection from the surface as well as the importance of the defect reservoir to follow, or not, the Ostwald ripening process. This has intriguingly been reported to occur only at certain processing conditions, Claverie et al. (2010). 


\section{Bibliography}

Amar, J. (2006). The Monte Carlo method in science and engineering. Computing in Science Engineering, 8(2):9-19.

Aziz, M. J., Sabin, P. C., and Lu, G.-Q. (1991). The activation strain tensor: Nonhydrostatic stress effects on crystal-growth kinetics. Physical Review B, 44:9812-9816.

Bai, G. and Nicolet, M.-A. (1991). Defects production and annealing in self-implanted si. Journal of applied physics, 70(2):649-655.

Barvosa-Carter, W. (1997). Stress Effects on Kinetics and Interfacial Roughening During Solid Phase Epitaxy. PhD thesis, Harvard University.

Berendsen, H., van der Spoel, D., and van Drunen, R. (1995). Gromacs: A message-passing parallel molecular dynamics implementation. Computer Physics Communications, 91(1$3): 43-56$.

Bernstein, N., Aziz, M., and Kaxiras, E. (2000). Atomistic simulations of solid-phase epitaxial growth in silicon. Physical Review B, 61(10):6696.

Blöchl, P. E., Smargiassi, E., Car, R., Laks, D. B., Andreoni, W., and Pantelides, S. T. (1993). First-principles calculations of self-diffusion constants in silicon. Physical Review Letters, 70:2435-2438.

Bonafos, C., Mathiot, D., and Claverie, A. (1998). Ostwald ripening of end-of-range defects in silicon. Journal of Applied Physics, 83(6):3008-3017. 
Boninelli, S., Impellizzeri, G., Alberti, A., Priolo, F., Cristiano, F., and Spinella, C. (2012a). Role of the ge surface during the end of range dissolution. Applied Physics Letters, 101(16):162103.

Boninelli, S., Impellizzeri, G., Priolo, F., Napolitani, E., and Spinella, C. (2012b). Fluorine in Ge: Segregation and EOR-defects stabilization. Nuclear Instruments and Methods in Physics Research Section B: Beam Interactions with Materials and Atoms, 282:21-24.

Bortz, A. B., Kalos, M. H., and Lebowitz, J. L. (1975). A new algorithm for monte carlo simulation of ising spin systems. Journal of Computational Physics, 17(1):10-18.

Bracht, H. (2000). Diffusion mechanisms and intrinsic point-defect properties in silicon. Materials Research Society Bulletin, 25(6):22.

Bracht, H. (2014). Defect engineering in germanium. Physica Status Solidi (a), 211(1):109.

Bracht, H., Haller, E., and Clark-Phelps, R. (1998). Silicon self-diffusion in isotope heterostructures. Physical Review Letters, 81(2):393.

Bracht, H., Schneider, S., Klug, J., Liao, C., Hansen, J. L., Haller, E., Larsen, A. N., Bougeard, D., Posselt, M., and Wündisch, C. (2009). Interstitial-mediated diffusion in germanium under proton irradiation. Physical Review Letters, 103(25):255501.

Buturla, E., Cottrell, P., Grossman, B., and Salsburg, K. (1981). Finite-element analysis of semiconductor devices: The fielday program. IBM Journal of Research and Development, $25(4): 218-231$.

Castrillo, P., Rubio, E., Pinacho, R., Martin-Bragado, I., Jaraiz, M., and Barbolla, J. (2001). Kinetic monte carlo process simulators: Bringing atomic scale knowledge to industrial fabrication. page 171. Trends on Nanotechnology.

Caturla, M.-J., de La Rubia, T. D., Marques, L., and Gilmer, G. (1996). Ion-beam processing of silicon at kev energies: A molecular-dynamics study. Physical Review B, $54(23): 16683$. 
Chao, Y.-L., Prussin, S., Woo, J., and Scholz, R. (2005). Preamorphization implantationassisted boron activation in bulk germanium and germanium-on-insulator. Applied Physics Letters, 87(14):142102.

Chroneos, A. and Bracht, H. (2014). Diffusion of n-type dopants in germanium. Applied Physics Reviews, 1(1):011301.

Claeys, C. and Simoen, E. (2008). Fundamental and technological aspects of extended defects in Germanium. Springer.

Claverie, A., Colombeau, B., De Mauduit, B., Bonafos, C., Hebras, X., Assayag, G. B., and Cristiano, F. (2003). Extended defects in shallow implants. Applied Physics A, 76(7):1025-1033.

Claverie, A., Koffel, S., Cherkashin, N., Benassayag, G., and Scheiblin, P. (2010). Amorphization, recrystallization and end of range defects in germanium. Thin Solid Films, $518(9): 2307$.

Claverie, A., Vieu, C., Faure, J., and Beauvillain, J. (1988). Cross-sectional high-resolution electron microscopy investigation of argon-ion implantation-induced amorphization of silicon. Journal of applied physics, 64(9):4415-4423.

Claverie, A., Vieu, C., Faure, J., and Beauvillain, J. (1989). Comparison between "intermediate"-and "heavy"-ion-bombardment-induced silicon amorphization at room temperature. Materials Science and Engineering: B, 2(1):99-104.

Cowern, N. E. B., Mannino, G., Stolk, P. A., Roozeboom, F., Huizing, H. G. A., van Berkum, J. G. M., Cristiano, F., Claverie, A., and Jaraiz, M. (1999). Energetics of self-interstitial clusters in Si. Physical Review Letters, 82(22):4460-4463.

Cowern, N. E. B., Simdyankin, S., Ahn, C., Bennett, N. S., Goss, J. P., Hartmann, J.-M., Pakfar, A., Hamm, S., Valentin, J., Napolitani, E., et al. (2013). Extended point defects in crystalline materials: Ge and si. Physical Review Letters, 110(15):155501.

Csepregi, L., Gyulai, J., and Lau, S. (1996). The early history of solid phase epitaxial growth. Mater. Chem. Phys., 46:178-180. 
Csepregi, L., Kennedy, E. F., Mayer, J. W., and Sigmon, T. W. (1978). Substrateorientation dependence of the epitaxial regrowth rate from si-implanted amorphous si. Journal of Applied Physics, 49:3906.

Csepregi, L., Küllen, R., Mayer, J., and Sigmon, T. (1977). Regrowth kinetics of amorphous ge layers created by ${ }^{74} \mathrm{Ge}$ and ${ }^{28} \mathrm{Si}$ implantation of ge crystals. Solid State Communications, 21(11):1019 - 1021 .

Csepregi, L., Mayer, J. W., and Sigmon, T. W. (1976). Regrowth behavior of ion-implanted amorphous layers on $<111>$ silicon. Applied Physics Letters, 29(2):92.

Darby, B., Yates, B., Martin-Bragado, I., Gomez-Selles, J., Elliman, R., and Jones, K. (2013). Substrate orientation dependence on the solid phase epitaxial growth rate of Ge. Journal of Applied Physics, 113(3):033505.

Darby, B., Yates, B., Rudawski, N., Jones, K., and Kontos, A. (2011). Self-implantation energy and dose effects on ge solid-phase epitaxial growth. Nuclear Instruments and Methods in Physics Research Section B: Beam Interactions with Materials and Atoms, 269(1):20-22.

Decoster, S. and Vantomme, A. (2009). Implantation-induced damage in Ge: strain and disorder profiles during defect accumulation and recovery. Journal of Physics D: Applied Physics, 42(16):165404.

Domain, C., Becquart, C., and Malerba, L. (2004). Simulation of radiation damage in fe alloys: an object kinetic monte carlo approach. Journal of Nuclear Materials, 335(1):121 -145 .

Dreizler, R. M. and Engel, E. (2011). Density functional theory. Springer.

Drosd, R. and Washburn, J. (1982). Some observations on the amorphous to crystalline transformation in silicon. Journal of Applied Physics, 53(1):397.

Duffy, R., Shayesteh, M., Kazadojev, I., and Yu, R. (2013). Germanium doping challenges. In Junction Technology (IWJT), 2013 13th International Workshop on, pages 16-21. IEEE. 
Duffy, R., Shayesteh, M., McCarthy, B., Blake, A., White, M., Scully, J., Yu, R., Kelleher, A.-M., Schmidt, M., Petkov, N., et al. (2011). The curious case of thin-body ge crystallization. Applied Physics Letters, 99(13):131910.

Eaglesham, D. J., Stolk, P. A., Gossmann, H. J., and Poate, J. M. (1994). Implantation and transient B diffusion in Si: The source of the interstitials. Applied Physics Letters, 65(18):2305.

Estreicher, S. K., Hastings, J. L., and Fedders, P. A. (1997). The ring-hexavacany in silicon: A stable and inactive defect. Applied Physics Letters, 70(4):432.

Fahey, P. M., Griffin, P., and Plummer, J. (1989). Point defects and dopant diffusion in silicon. Reviews of Modern Physics, 61(2):289.

Frisch, M. J., Trucks, G. W., Schlegel, H. B., Scuseria, G. E., Robb, M. A., Cheeseman, J. R., Scalmani, G., Barone, V., Mennucci, B., Petersson, G. A., Nakatsuji, H., Caricato, M., Li, X., Hratchian, H. P., Izmaylov, A. F., Bloino, J., Zheng, G., Sonnenberg, J. L., Hada, M., Ehara, M., Toyota, K., Fukuda, R., Hasegawa, J., Ishida, M., Nakajima, T., Honda, Y., Kitao, O., Nakai, H., Vreven, T., Montgomery, Jr., J. A., Peralta, J. E., Ogliaro, F., Bearpark, M., Heyd, J. J., Brothers, E., Kudin, K. N., Staroverov, V. N., Kobayashi, R., Normand, J., Raghavachari, K., Rendell, A., Burant, J. C., Iyengar, S. S., Tomasi, J., Cossi, M., Rega, N., Millam, J. M., Klene, M., Knox, J. E., Cross, J. B., Bakken, V., Adamo, C., Jaramillo, J., Gomperts, R., Stratmann, R. E., Yazyev, O., Austin, A. J., Cammi, R., Pomelli, C., Ochterski, J. W., Martin, R. L., Morokuma, K., Zakrzewski, V. G., Voth, G. A., Salvador, P., Dannenberg, J. J., Dapprich, S., Daniels, A. D., Farkas, A., Foresman, J. B., Ortiz, J. V., Cioslowski, J., and Fox, D. J. (2009). Gaussian 09. Gaussian Inc. Wallingford CT 2009.

Fu, C.-C., Torre, J. D., Willaime, F., Bocquet, J.-L., and Barbu, A. (2005). Multiscale modelling of defect kinetics in irradiated iron. Nature Materials, 4:68-74.

Furuno, S., Izui, K., and Otsu, H. (1976). Defect clusters in germanium crystals irradiated with electrons in a high voltage electron microscope. Japanese Journal of Applied Physics, 15(5):889. 
Ghoniem, N., M, Tong, S.-H., and Sun, L. (2000). Parametric dislocation dynamics: a thermodynamics-based approach to investigations of mesoscopic plastic deformation. Physical Review B, 61(2):913.

Gillespie, D. T. (1976). A general method for numerically simulating the stochastic time evolution of coupled chemical reactions. Journal of Computational Physics, 22:403.

Goldstein, H. (1957). Classical mechanics. Pearson Education India.

Gomez-Selles, J., Darby, B., Jones, K., and Martin-Bragado, I. (2014). Lattice kinetic Monte Carlo modeling of germanium solid phase epitaxial growth. physica status solidi (c), 11(1):93-96.

Gomez-Selles, J. L., Claverie, A., Sklenard, B., Benistant, F., and Martin-Bragado, I. (2015). Atomistic simulation of damage accumulation and amorphization in Ge. Journal of Applied Physics, 117(5):055703.

Guo, B., Variam, N., Jeong, U., Mehta, S., Posselt, M., and Lebedev, A. (2002). Experimental and simulation studies of the channeling phenomena for high energy implantation. In Ion Implantation Technology. 2002. Proceedings of the 14 th International Conference on, pages 131-134.

Haile, J. (1992). Molecular dynamics simulation, volume 27. Wiley, New York.

Hernandez-Mangas, J. M. (2000). Simulacion BCA de la Implantacion ionica en materiales cristalinos: mejora de los modelos fisicos y del tiempo de calculo. PhD thesis, University of Valladolid.

Hickey, D., Bryan, Z., Jones, K., Elliman, R., and Haller, E. (2008). Defects in Ge and Si caused by $1 \mathrm{MeV} \mathrm{Si}^{+}$implantation. Journal of Vacuum Science \& Technology B: Microelectronics and Nanometer Structures, 26(1):425-429.

Hockney, R. W. and Eastwood, J. W. (1988). Computer simulation using particles. Adam Hilger by IOP Publishing LTD, 335 East 45th Street, New York, NY 10017-3483, USA. ISBN 0-85274-392-0. 
Impellizzeri, G., Mirabella, S., Bruno, E., Piro, A., and Grimaldi, M. (2009a). B activation and clustering in ion-implanted Ge. Journal of applied Physics, 105(6):063533.

Impellizzeri, G., Mirabella, S., and Grimaldi, M. (2011). Ion implantation damage and crystalline-amorphous transition in Ge. Applied Physics A, 103(2):323-328.

Impellizzeri, G., Mirabella, S., Irrera, A., Grimaldi, M., and Napolitani, E. (2009b). Gaimplantation in ge: Electrical activation and clustering. Journal of Applied Physics, 106(1):013518.

ITRS (2013). International technology roadmap for semiconductors, 2013 edition. http://public.itrs.net/.

Jaraiz, M. (2004). Predictive Simulation of Semiconductor Processing, chapter Atomistic Simulations in Materials Processing, pages 73-109. Springer-Verlag, Berlin.

Johnson, M. D., Caturla, M. J., and de la Rubia, T. D. (1998). A kinetic monte-carlo study of the effective diffusivity of the silicon self-interstitial in the presence of carbon and boron. Journal of Applied Physics, 84(4):1963-7.

Katz, L. and Sze, S. (1983). VLSI technology. McGraw-Hill, Singapore.

Keating, P. (1966). Effect of invariance requirements on the elastic strain energy of crystals with application to the diamond structure. Physical Review, 145(2):637.

Koffel, S., Cherkashin, N., Houdellier, F., Hytch, M., Benassayag, G., Scheiblin, P., and Claverie, A. (2009). End of range defects in Ge. Journal of Applied Physics, 105(12):126110.

Kresse, G. and Hafner, J. (1993). Ab initio molecular dynamics for liquid metals. Physical Review $B, 47: 558$.

Kube, R., Bracht, H., Hüger, E., Schmidt, H., Hansen, J. L., Larsen, A. N., Ager, J. W., Haller, E. E., Geue, T., and Stahn, J. (2013). Contributions of vacancies and selfinterstitials to self-diffusion in silicon under thermal equilibrium and nonequilibrium conditions. Physical Review B, 88:085206. 
Landau, D. P. and Binder, K. (2000). A Guide to Monte Carlo Simulations in Statistical Physics. Cambridge University Press.

Lim, D. R.; Rafferty, C. S. K. F. P. (1995). The role of the surface in transient enhanced diffusion. Applied Physics Letters, 67.

Lindhard, J. and Scharff, M. (1961). Energy dissipation by ions in the kev region. Physical Review, 124(1):128.

Lindhard, J., Scharff, M., and Schiøtt, H. E. (1963). Range concepts and heavy ion ranges. Munksgaard.

Lu, G., Nygren, E., Aziz, M., Turnbull, D., and White, C. (1990). Pressure-enhanced solid phase epitaxy of germanium. Applied Physics Letters, 56(2):137-139.

Lu, G.-Q., Nygren, E., and Aziz, M. J. (1991). Pressure-enhanced crystallization kinetics of amorphous Si and Ge: Implications for point-defect mechanisms. Journal of Applied Physics, 70(10):5323-5345.

Martin-Bragado, I. (2011). \{111\} local configurations: The main source of silicon defects during solid phase epitaxial regrowth modeled by lattice kinetic monte carlo. Applied Physics Letters, 98:233109.

Martin-Bragado, I. (2012). Importance of twin defect formation created by solid-phase epitaxial growth: An atomistic study. Scripta Materialia, 66:186-189.

Martin-Bragado, I. (2013). Comprehensive modeling of solid phase epitaxial growth using lattice kinetic monte carlo. Nuclear Instruments and Methods in Physics Research Section B: Beam Interactions with Materials and Atoms, 303(0):184 - 187.

Martin-Bragado, I., Avci, I., Zographos, N., Jaraiz, M., and Castrillo, P. (2008). From point defects to dislocation loops: a comprehensive modelling framework for self interstitial defects in silicon. Solid-State Electronics, 52:1430-1436.

Martin-Bragado, I., Jaraiz, M., Castrillo, P., Pinacho, R., Rubio, J., and Barbolla, J. (2004). Ion implant simulations: Kinetic monte carlo annealing assessment of the dominant features. Applied physics letters, 84(24):4962-4964. 
Martin-Bragado, I. and Moroz, V. (2009). Facet formation during solid phase epitaxy regrowth: A lattice kinetic monte carlo model. Applied Physics Letters, 95(12):123123.

Martin-Bragado, I. and Moroz, V. (2011). Modeling of $\{311\}$ facets using a lattice kinetic monte carlo 3-dimensional model for selective epitaxial growth of silicon. Applied Physics Letters, 98:153111.

Martin-Bragado, I., Rivera, A., Valles, G., Gomez-Selles, J. L., and Caturla, M. J. (2013). MMonCa: An Object Kinetic Monte Carlo simulator for damage irradiation evolution and defect diffusion. Computer Physics Communications, 184(12):2703-2710.

Martin-Bragado, I. and Sklenard, B. (2012). Understanding Si(111) solid phase epitaxial regrowth using monte carlo modeling: Bi-modal growth, defect formation, and interface topology. Journal of Applied Physics, 112(2):024327.

Martin-Bragado, I., Tian, S., Johnson, M., Castrillo, P., Pinacho, R., Rubio, J., and Jaraiz, M. (2006). Modelling charge defects, dopant diffusion and activation mechanisms for TCAD simulations using kinetic monte carlo. Nucl. Instr. Meth. B, 253:63-67.

Mazzone, A. and Servidori, M. (1988). Properties of clustered interstitials in ion-implanted silicon: Comparison between a Monte Carlo simulation and x-ray diffraction measurements. Philosophical magazine letters, 57(2):85-90.

Mirabella, S., Bruno, E., Priolo, F., De Salvador, D., Napolitani, E., Drigo, A., and Carnera, A. (2003). Dissolution kinetics of boron-interstitial clusters in silicon. Applied physics letters, 83(4):680-682.

Mirabella, S., De Salvador, D., Napolitani, E., Bruno, E., and Priolo, F. (2013). Mechanisms of boron diffusion in silicon and germanium. Journal of Applied Physics, 113(3):031101.

Mitchell, E. W. J. (1957). The effect of radiation damage on the electronic properties of solids. British Journal of Applied Physics, 8(5):179. 
Mok, K., Benistant, F., Jaraiz, M., Rubio, J., Castrillo, P., Pinacho, R., and Srinivasan, M. (2008). Comprehensive model of damage accumulation in silicon. Journal of Applied Physics, 103(1):014911.

Moore, G. E. et al. (1998). Cramming more components onto integrated circuits. Proceedings of the IEEE, 86(1):82-85.

Morehead Jr, F. and Crowder, B. (1970). A model for the formation of amorphous Si by ion bombardment. Radiation Effects, 6(1):27-32.

Mueller, D. C., Alonso, E., and Fichtner, W. (2003). Arsenic deactivation in si: Electronic structure and charge states of vacancy-impurity clusters. Physical Review B, 68(4):045208.

Napolitani, E., Bruno, E., Bisognin, G., Mastromatteo, M., De Salvador, D., Scapellato, G., Boninelli, S., Priolo, F., Privitera, V., and Carnera, A. (2014). Role of ion mass on damage accumulation during ion implantation in Ge. Physica Status Solidi (a), 211(1):118-121.

Oen, O. S. and Robinson, M. T. (1976). Computer studies of the reflection of light ions from solids. Nuclear instruments and Methods, 132:647-653.

Olson, G. and Roth, J. (1988). Kinetics of solid phase crystallization in amorphous silicon. Materials Science Reports, 3(1):1-77.

Ostwald, W. (1900). Über die vermeintliche isomerie des roten und gelben quecksilberoxyds und die oberflächenspannung fester körper. Z. phys. Chem, 34:495-503.

Panciera, F., Fazzini, P., Collet, M., Boucher, J., Bedel, E., and Cristiano, F. (2010). Endof-range defects in germanium and their role in boron deactivation. Applied Physics Letters, 97(1):012105.

Pelaz, L., Jaraiz, M., Gilmer, G. H., Gossmann, H.-J., Rafferty, C. S., Eaglesham, D., and Poate, J. M. (1997). B diffusion and clustering in ion implanted Si: The role of B cluster precursors. App. Phys. Lett., 70(17):2285-2287. 
Pelaz, L., Marqués, L. A., and Barbolla, J. (2004). Ion-beam-induced amorphization and recrystallization in silicon. Journal of applied physics, 96(11):5947-5976.

Pichler, P. (2004). Intrinsic point defects, impurities and their diffusion in Silicon. Springer Wien.

Pinacho, R., Jaraiz, M., Castrillo, P., Martin-Bragado, I., Rubio, J. E., and Barbolla, J. (2005). Modeling arsenic deactivation through arsenic-vacancy clusters using an atomistic kinetic monte carlo approach. Applied Physics Letters, 86:252103.

Plimpton, S. (1995). Fast parallel algorithms for short-range molecular dynamics. Journal of Computational Physics, 117:1-19.

Posselt, M. (2015). Private communication.

Posselt, M., Bischoff, L., Grambole, D., and Herrmann, F. (2006). Competition between damage buildup and dynamic annealing in ion implantation into Ge. Applied physics letters, 89(15):151918.

Posselt, M., Gao, F., and Bracht, H. (2008). Correlation between self-diffusion in Si and the migration mechanisms of vacancies and self-interstitials: An atomistic study. Physical Review B, 78:035208.

Rechtin, M., Pronko, P., Foti, G., Csepregi, L., Kennedy, E., and Mayer, J. (1978). An electron microscopy study of defect structures in recrystallized amorphous layers of selfion-irradiated $<111>$ silicon. Philosophical Magazine A, 37(5):605-620.

Robinson, M. T. and Torrens, I. M. (1974). Computer simulation of atomic-displacement cascades in solids in the binary-collision approximation. Physical Review B, 9(12):5008.

Rudawski, N., Jones, K., and Gwilliam, R. (2008). Stressed solid-phase epitaxial growth of ion-implanted amorphous silicon. Materials Science and Engineering: R: Reports, $61(1-6): 40-58$.

Satta, A., Simoen, E., Clarysse, T., Janssens, T., Benedetti, A., De Jaeger, B., Meuris, M., and Vandervorst, W. (2005). Diffusion, activation, and recrystallization of boron 
implanted in preamorphized and crystalline germanium. Applied Physics Letters, 87(17):172109-172109.

Sentaurus (2012). Sentaurus Process user guide. Synopsys Inc. Mountain View, CA, USA.

Sklenard, B., Barbe, J.-C., Batude, P., Rivallin, P., Tavernier, C., Cristoloveanu, S., and Martin-Bragado, I. (2013). An atomistic investigation of the impact of in-plane uniaxial stress during solid phase epitaxial regrowth. Applied Physics Letters, 102(15):151907.

Sklenard, B., Barbe, J.-C., Batude, P., Rivallin, P., Tavernier, C., Cristoloveanu, S., and Martin-Bragado, I. (2014). Atomistic investigation of the impact of stress during solid phase epitaxial regrowth. physica status solidi (c), 11(1):97-100.

Soler, J. M., Artacho, E., Gale, J. D., Garcìa, A., Junquera, J., Ordejón, P., and SánchezPortal, D. (2002). The SIESTA method for ab initio order- N materials simulation. Journal of Physics: Condensed Matter, 14(11):2745.

Śpiewak, P., Vanhellemont, J., Sueoka, K., Kurzydłowski, K. J., and Romandic, I. (2008). First principles calculations of the formation energy and deep levels associated with the neutral and charged vacancy in germanium. Journal of Applied Physics, 103(8):086103.

Spyrakos, C. C. (1994). Finite Element Modeling. West Virginia Univ. Press.

Stolk, P. A., Gossmann, H. J., Eaglesham, D. J., Jacobson, D. C., Rafferty, C. S., Gilmer, G. H., Jaraiz, M., Poate, J. M., Luftman, H. S., and Haynes, T. E. (1997). Physical mechanism of transient enhanced dopant diffusion in ion-implanted silicon. Journal of Applied Physics, 81:6031-6050.

Stoller, R. E., Toloczko, M. B., Was, G. S., Certain, A. G., Dwaraknath, S., and Garner, F. A. (2013). On the use of SRIM for computing radiation damage exposure. Nuclear Instruments and Methods in Physics Research Section B: Beam Interactions with Materials and Atoms, 310:75-80.

Stukowski, A. (2010). Visualization and analysis of atomistic simulation data with ovitothe open visualization tool. Modelling and Simulation in Materials Science and Engineering, 18(1):015012. 
Sueoka, K. and Vanhellemont, J. (2006). Ab initio studies of intrinsic point defects, interstitial oxygen and vacancy or oxygen clustering in germanium crystals. Materials science in semiconductor processing, 9(4):494-497.

Sze, S. M. and Ng, K. K. (2006). Physics of semiconductor devices. John Wiley \& Sons.

Vanhellemont, J., Śpiewak, P., and Sueoka, K. (2007). On the solubility and diffusivity of the intrinsic point defects in germanium. Journal of applied physics, 101(3):036103.

Vieu, C., Claverie, A., Faure, J., and Beauvillain, J. (1992). In Phase transformation kinetics in thin films.

Vineyard, G. H. (1957). Frequency factors and isotope effects in solid state rate processes. Journal of Physics and Chemistry of Solids, 3:121-127.

Voter, A. (2007). Introduction to the kinetic monte carlo method. In Sickafus, K., Kotomin, E., and Uberuaga, B., editors, Radiation Effects in Solids, volume 235 of NATO Science Series, pages 1-23. Springer Netherlands.

Williams, J. S. and Elliman, R. G. (1983). Role of electronic processes in epitaxial recrystallization of amorphous semiconductors. Physical Review Letters, 51:1069-1072.

Wittmann, R. and Selberherr, S. (2007). A study of ion implantation into crystalline germanium. Solid-state electronics, 51(6):982-988.

Yates, B., Darby, B., Petersen, D. H., Hansen, O., Lin, R., Nielsen, P., Romano, L., Doyle, B., Kontos, A., and Jones, K. (2012). Activation and thermal stability of ultra-shallow $\mathrm{B}^{+}$-implants in ge. Journal of Applied Physics, 112(12):123525.

Zbib, H. M., Rhee, M., and Hirth, J. P. (1998). On plastic deformation and the dynamics of 3D dislocations. International Journal of Mechanical Sciences, 40(2):113-127.

Ziegler, J. F., Biersack, J. P., and Ziegler, M. D. (2008). SRIM, the stopping and range of ions in matter. SRIM Co. 


\section{List of Figures}

1.1 Qualitative representation of the impact caused by varying the energy of the \begin{tabular}{|c|}
\hline implanted ions (blue dashed line) or the ion fluence (red dashed line), when \\
\hline
\end{tabular}

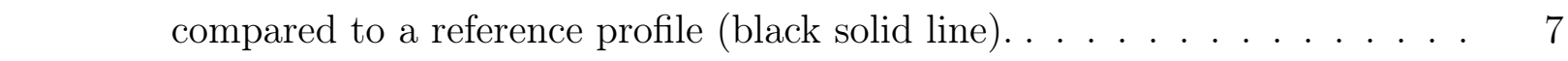

1.2 Sketches for $I$ (red) and $V$ (gray) formation. (a) Frenkel-pair formation within the bulk by thermal vibrations. (b) Atom displacement due to the impact of an incoming ion (blue). Lattice atoms are green-colored. Arrows indicate possible trajectories of the atoms. . . . . . . . . . . . . 9 9

1.3 Sketches for $I$ (red) and $V$ (gray) emission and recombination in the surface. As a example, the interface between bulk lattice Si (green) atoms and an amorphous Silicon Oxide (SiOx) layer (blue) atoms is schematically represented for (a) Interstitial atoms emission and/or recombination in the $\mathrm{Si} / \mathrm{SiOx}$ interface, and (b) Vacancy sites emission and/or recombination in

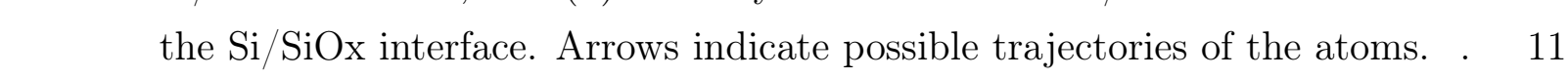

1.4 Cross TEM observation of $I$-type Extended Defects in Ge produced after implantation of $2 \times 10^{14} 100 \mathrm{keV}$ Ge ions and annealed at $340^{\circ} \mathrm{C}$ for 1 hour. The inset in the left shows a high resolution image of an irregular small

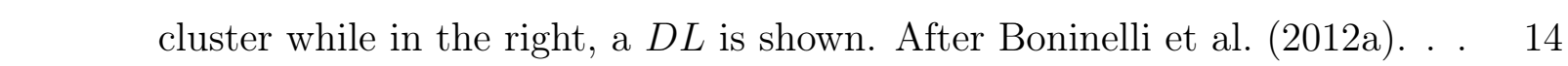

1.5 Different c-RBS measurements taken after implanting $160 \mathrm{keV}$ Ar ions at different fluences into Ge samples. (a) is the Normalized Yield and (b) is the defect fraction caused at every condition. After Decoster and Vantomme

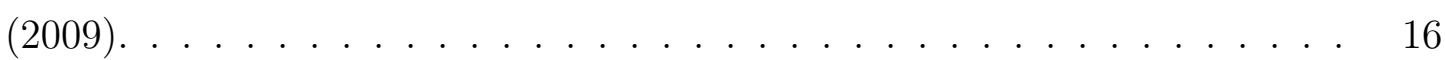


1.6 XTEM observation of the damage accumulation, amorphization and further extension of the amorphous layer caused by $150 \mathrm{keV}$ Ge ions implanted into Ge samples for increasing doses, from a) to e). After Claverie et al. (2010).

1.7 XTEM observation of Ge SPER process after $330^{\circ} \mathrm{C}$ anneal for different times, from (a) to (d). The $\alpha / \mathrm{c}$ interface is indicated with an arrow. After

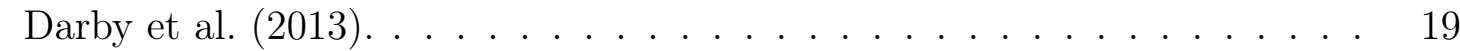

1.8 Set of PVTEM images of the defects caused by ion implantation in Ge and their thermal evolution for subsequent anneals: a), b) and c), taken from Claverie et al. (2010). . . . . . . . . . . . . . . . . . 21

1.9 Indicative diagram of the simulation techniques used in materials science. Different simulation techniques are represented and arranged depending on the time and space scale which are usually capable of reproduce (x-axis), and the quantity of approximations, inverse to the level of detail achieved (y-axes). From the smallest scale to the biggest, Density Functional Theory (DFT), Molecular Dynamics (MD), Kinetic Monte Carlo (KMC), Dislocation Dynamics (DD), and the Finite Element Method (FEM) are represented by schematic representations. . . . . . . . . . . . . . . . . 22

2.1 Energetic diagram for a transition between two states: $i$ and $j$. Formation and barrier energies are represented. After Martin-Bragado et al.[(2013)] . . 32

2.2 Overall structure of MMonCa. The user interface relies on a layer of the TCL interpreter, extended to support the simulator. The extension relies on specialized modules to control the space, time and defects. Several defects are supported as the objects to be simulated: clusters (C), mobile particles (MP) and interfaces (Int). . . . . . . . . . . . . . . . 34

2.3 The KMC algorithm contains a list of all the transitions associated with the objects being simulated, and picks the next one proportionally to such rates. That can be seen graphically as getting a random number uniformly distributed in $\left[0, R_{N}\right)$ and picking up the event "aligned" with such number. After Martin-Bragado et al.](2013). . 
2.4 Space is divided into small prismatic elements using a tensor mesh. a) An interface is the union of all element faces between adjacent different materials

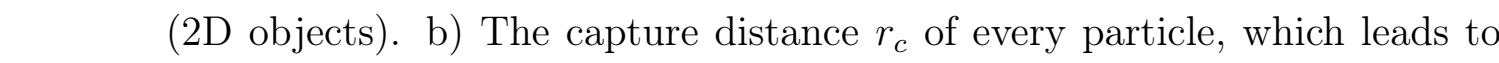
a 3D capture volume, is defined independently. c) The capture distance of clusters is built as the union of the capture distances of their constituent particles. . . . . . . . . . . . . . . . . . 36

2.5 Example of the objects to be simulated by OKMC. a) High magnification image from a XTEM micrograph of a dislocation loop formed in a Ge sample implanted with $30 \mathrm{keV} 2 \times 10^{14} \mathrm{Ge} / \mathrm{cm}^{2}$ after annealing at $340 \mathrm{C}$ for $1 \mathrm{~h}$ (after Boninelli et al. $(2012 \mathrm{a}))$. In b) the objects that are to be simulated are highlighted. In this case, Is (red) and $V$ s (green) as mobile particles, and the dislocation loop as a cluster formed by $I \mathrm{~s}$. Assuming that the lattice influence in the defect behavior is included the rates, OKMC takes

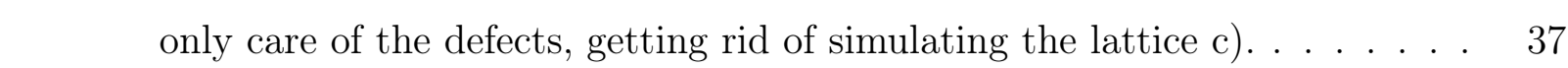

2.6 Three phase segregation model. Particles can be captured and emitted at either side, but the binding energies, migration energies and capture barriers might be different at each side. After Martin-Bragado et al.[(2013). . . . . 39

2.7 Comparison between KMC (symbols) and the theoretical solution (lines) for the time evolution of a spatial concentration of non-interacting particles

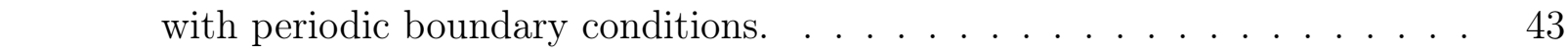

2.8 Concentration of Si $I$ s in $\{311\}$ defects as a function of time for three different temperatures after a $40 \mathrm{keV}, 2 \times 10^{13} \mathrm{~cm}^{-2}$ Si into Si implant. Symbols: Experimental data taken from Ref.|Eaglesham et al.[1994); Stolk et al. (1997), lines: simulation results using MMonCa. . . . . . . . . . . . . 45

2.9 Interstitial supersaturation as a function of time after a $40 \mathrm{keV}, 2 \times 10^{13} \mathrm{~cm}^{-2}$ Si into Si implantation at different temperatures 600,700 and $800^{\circ} \mathrm{C}$. Sym\begin{tabular}{|c|}
\hline bols: Experimental data taken from Cowern et al.[(1999), lines: simulation \\
\hline
\end{tabular} results using MMonCa. . . . . . . . . . . . . . . . . . . . . . . . . 46 
3.1 Sketch of the lattice atoms (in green) arranged in the crystalline phase (red

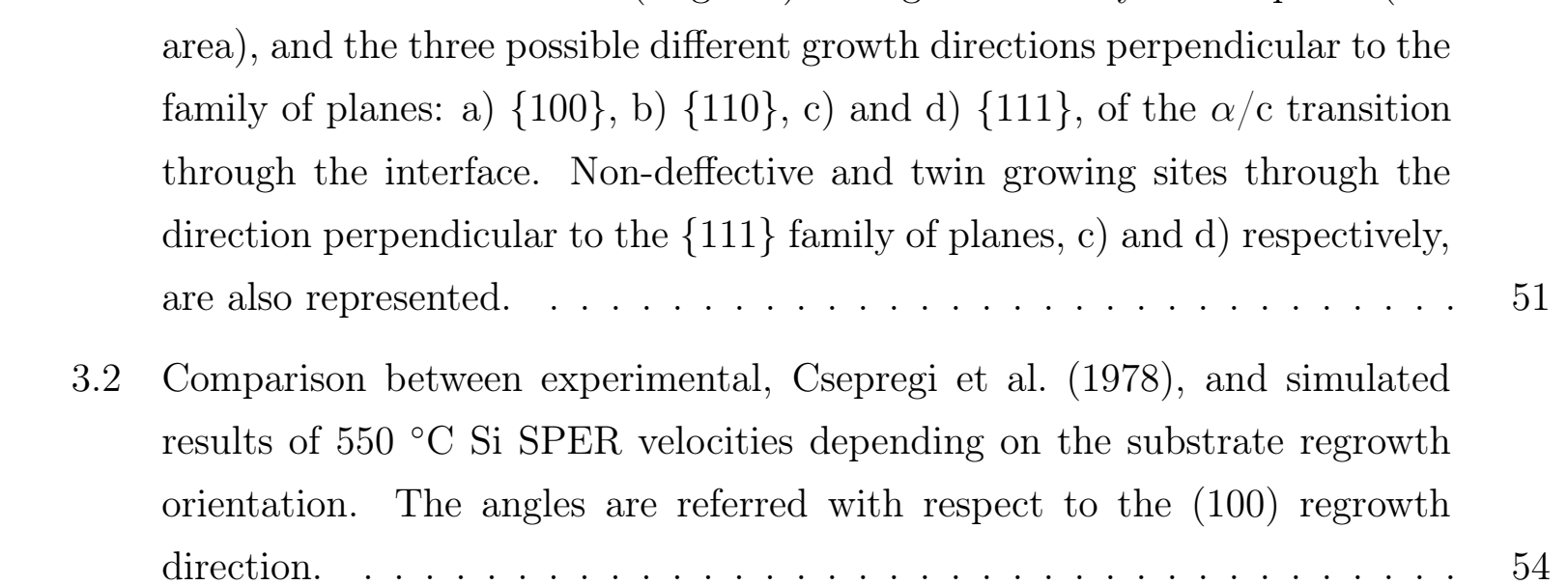

3.3 Simulated (dashed lines), Martin-Bragado and Sklenard $(2012)$, and exper\begin{tabular}{|l|}
\hline imental (thin solid lines and symbols), Csepregi et al. [1976), results of \\
\hline regrown distance with time for $\mathrm{Si}(100), \mathrm{Si}(110)$ and $\mathrm{Si}(111)$ substrates. Two \\
\hline different velocity regimes are found for $\mathrm{Si}(111)$, where a) a mean between \\
\hline simulations performed for different random seeds is presented, and b) Ex- \\
\hline amples of the results obtained with those different seeds are shown in blue \\
\hline dashed lines. . . . . . . . . . . . . . . . . . . . . . . . . . . 55 \\
\hline
\end{tabular}

3.4 Atomistic representation of the growing process caused by the engagement of a [111] facet, a) (in red) and b) (in green) [1-11]. For the specific config\begin{tabular}{|c|}
\hline uration shown here, both facets can join (red-green bonds) and grow faster \\
\hline by a mixed $\{110\}$ microscopic event (blue dashed line), which serves as an
\end{tabular} unexpected seed for the growth of the crystal. . . . . . . . . . . . 56

3.5 Normalized regrowth velocity of the $\mathrm{Si}(100)$ interface as a function of hydrostatic pressure at $530{ }^{\circ} \mathrm{C}$. LKMC simulations results (dashed line) are \begin{tabular}{|c|c|c|c|}
\hline compared with experimental measurements of & Lu et al. [1991). After Skle-
\end{tabular} nard et al. (2014). . . . . . . . . . . . . . . . . . 57

$5.1 \quad$ 2D-sketch of the amorphization process. Step $1 \ldots \ldots$. . . . . . . 70

5.2 2D-sketch of the Amorphization process. Step 2 . . . . . . . . . . . . . 71

$5.3 \quad$ Amorphization process for increasing ion dose . . . . . . . . . . . . . . 72 
5.4 Activation energies of recombination as a function of the Amorphous Pocket

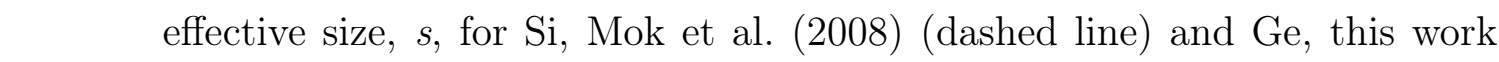
(solid line). SPER energies are also shown for Si, Olson and Roth (1988);

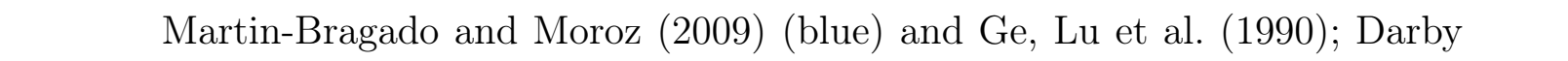
et al. $(2013)$ (red). . . . . . . . . . . . . . . . . . . . 74

5.5 Displaced Ge atoms for different $35 \mathrm{keV}$ B fluences at $\mathrm{LN}_{2}$ T. Symbols represent experimental data.Impellizzeri et al.(2009a) Simulation results with \begin{tabular}{|c|}
\hline (solid line) and without (dashed line) the amorphization model are pre- \\
\hline
\end{tabular} sented. . . . . . . . . . . . . . . . 76

5.6 Maximum defect fraction for increasing fluences for all the ions studied in this work. The three different regions of damage accumulation are also shown. 77

$5.7 A P$ recombination rates, $\nu$, as a function of their effective size, $s$, for Si,

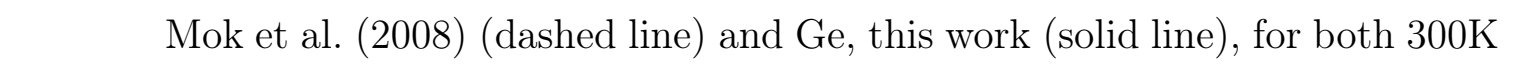
and 500K. Corresponding temperatures are indicated with arrows between ․ Si and Ge lines. . . . . . . . . . . . . . . . . . . . 81

5.8 Comparison of the damage profiles calculated with BCA assuming an amor\begin{tabular}{|c|}
\hline phous (blue) or a crystalline target (red) for (a) $35 \mathrm{keV}, 1 \times 10^{14} \mathrm{~B} / \mathrm{cm}^{-2}$ \\
\hline
\end{tabular}

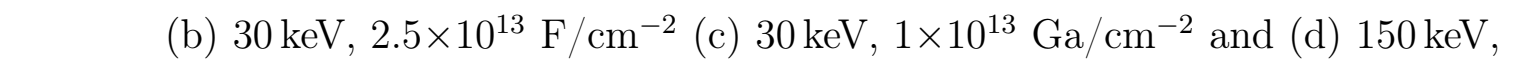

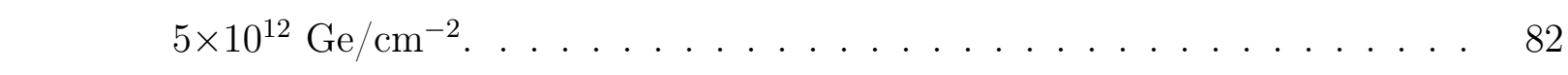

$5.9 \quad \alpha /$ c damage ratios for the studied ions. $\ldots \ldots \ldots$. . . . . . . . 83

$5.102 \mathrm{D}$ Concentration distribution histogram of $A P$ composition, $I_{n} V_{m}$, resulting after simulations of $150 \mathrm{keV}$ Ge (a) and $40 \mathrm{keV} \mathrm{B} \mathrm{(b)} \mathrm{implants} \mathrm{at} \mathrm{LN}_{2} \mathrm{~T}$. (c) is the normalized cumulative damage distribution of the same data for both Ge (solid line) and B (dashed line) implantation as a function of the number of defects forming an $A P$. Fluences where chosen to generate the same amount of damage $(I \mathrm{~s}$ and $V \mathrm{~s})$ for both conditions. . . . . . . . . . 84

5.11 Damage profiles caused by $150 \mathrm{keV} 2.25 \times 10^{13} \mathrm{Ge} / \mathrm{cm}^{2}$ (red lines) and $40 \mathrm{keV}$ $5 \times 10^{14} \mathrm{~B} / \mathrm{cm}^{2}$ implants (black lines) at both $\mathrm{LN}_{2} \mathrm{~T}$ and $0^{\circ} \mathrm{C}$. Vertical arrows show the quantity of dynamically annealed damage when comparing the cryogenic implantations with those performed at $0^{\circ} \mathrm{C}$. The amorphization threshold is also represented (blue dashed line). . . . . . . . . . . . 86 
5.12 Location of $\alpha / c$ interfaces measured experimentally (y-axis) for $150 \mathrm{keV} \mathrm{Ge}$

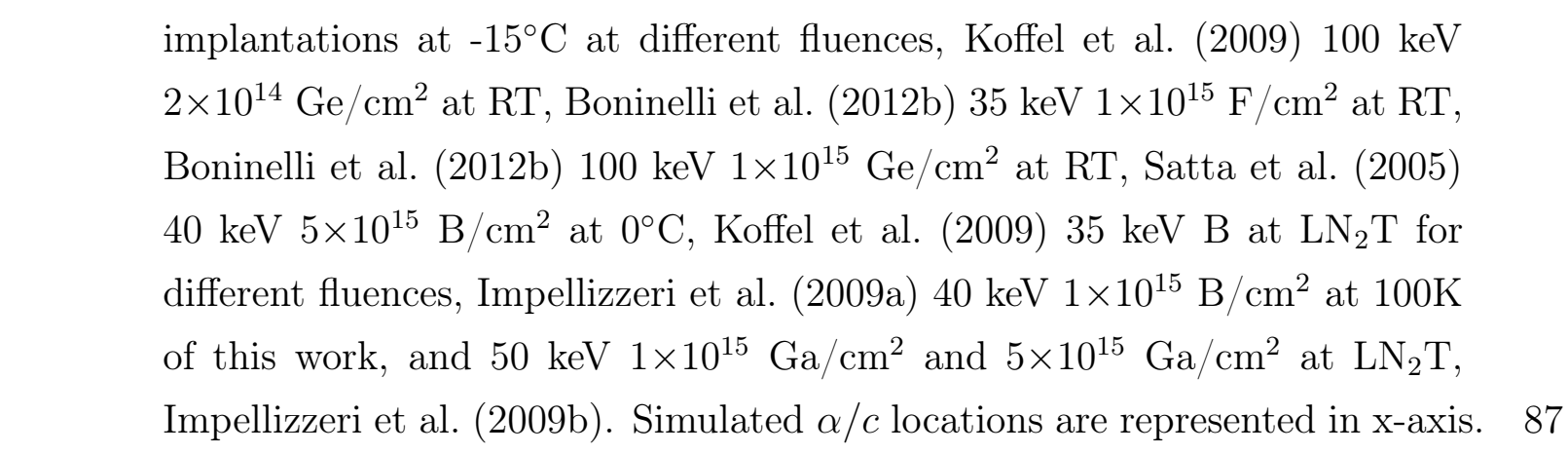

5.13 Displaced Ge atoms for different $35 \mathrm{keV}$ B fluences at RT. Symbols represent \begin{tabular}{|c|}
\hline experimental data, Impellizzeri et al.[(2009a). Simulation results (lines) for \\
\hline different fluxes are also presented along with results obtained without cluster \\
\hline
\end{tabular} dissolutions. . . . . . . . . . . . . . . . . . . . 88

5.14 Experimental measurements, Posselt et al. [2006) (symbols) and simulated \begin{tabular}{|c|}
\hline (lines) of the relative disorder induced in the Ge lattice after $30 \mathrm{keV} \mathrm{Ga}$ \\
\hline implantations at both RT and $250^{\circ} \mathrm{C}$ for both $10^{12} \mathrm{~cm}^{-2} \mathrm{~s}^{-1}$ and $10^{19} \mathrm{~cm}^{-2} \mathrm{~s}^{-1}$ \\
\hline
\end{tabular} - fluxes. . . . . . . . . . . . . . . . . . . . . . . 90

6.1 Comparison between experimental results Darby et al. (2013) (solid lines) and LKMC simulations (dashed lines) for the three main regrowth directions: [001], [011] and [111]. The first $200 \mathrm{~nm}$ of Ge SPER at $330^{\circ} \mathrm{C}$ are shown. A linear regression in the [011] configuration has been done to fit the simulation of that orientation. . . . . . . . . . . . . . . . . . . . . 99

6.2 Final atomistic configurations of the $150 \mathrm{~nm}$ of simulated SPER correspond\begin{tabular}{|c|c|c|c|}
\hline ing to the results of Figure & 6.1 . Only Ge $\alpha / \mathrm{c}$ interface and defective- \\
\hline
\end{tabular} positioned atoms (twins) are plotted. . . . . . . . . . . . 100

$6.3 \quad$ Ge $\alpha / \mathrm{c}$ interface and defective-positioned atoms (twins) of an isochronal \begin{tabular}{|c|}
\hline anneal for eight different configurations at $330^{\circ} \mathrm{C}$ and $1000 \mathrm{~s}$. a) corresponds \\
\hline
\end{tabular}

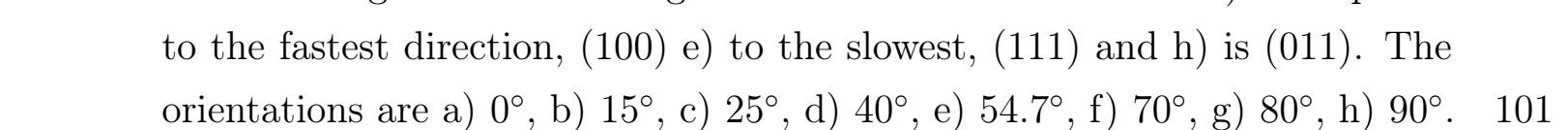


6.4 Comparison of experimental Darby et al. (2013) Ge SPER velocities (solid line) and LKMC Simulations (dashed line) for a range of orientations between $0^{\circ}$ and $90^{\circ}$ at $330^{\circ} \mathrm{C}$. . . . . . . . . . . . . . . . . . 102

6.5 Comparison between simulations using the calibration presented in Darby et al. [(2013) and experimental results Csepregi et al.[(1977); Lu et al.](1990) along with the fitted Arrhenius law of Claverie et al. [(2010) for Ge SPER velocity as a function of the process temperatures. . . . . . . . . . . . . . 104

$\begin{array}{lll}6.6 & \text { Ge SPER velocity as a function of the pressure for different temperatures. } & 105\end{array}$

6.7 Planarization process for different ME volume sizes. . . . . . . . . . . . . . 106

6.8 Atomistic view of a planarization process. . . . . . . . . . . . . 107

$\begin{array}{lll}6.9 & \text { Ge SPER velocity as a function of the pressure for different temperatures. } & 109\end{array}$ 


\section{List of Tables}

$1.1 \quad$ Band Gap, and carrier mobilities of Si and Ge, Sze and Ng[(2006)] . . . . . 5

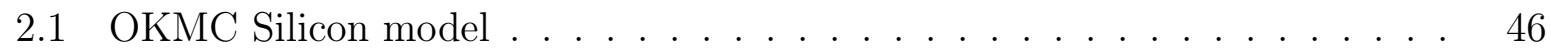

3.1 Prefactors and activation energies for each local recrystallization configuration for Si. After Martin-Bragado(2013). . . . . . . . . . . . . . . . . . . 54

3.2 Values of the components of the activation strain tensors depending on the number of crystalline first neighbors (c1NN) in Si. $\Omega_{S i}$ is the molar volume of Si. After Sklenard et al. $(2014) . \ldots$. . . . . . . . . . . . . . . . 58

$5.1 \quad$ Migration and formation energies $\left(E_{m}, E_{f}\right)$ and prefactors $\left(D_{0, m}, D_{0, f}\right)$ of Ge $I \mathrm{~s}$ and $V_{\mathrm{s}}$ used in this model. . . . . . . . . . . . . . . . . . . . . . . 74

$5.2 \quad c$ - to $\alpha$-cascade transitions used for all the studied conditions in this work, and transitions between the three different regions of damage accumulation in the peak of maximum damage, extracted from Fig. 5.6 ( (all means conditions used in this study). . . . . . . . . . . . . . . . . 78

6.1 Recrystallization prefactors for the local configurations. $h$ and $l$ stand for high and low recrystallization rate, depending on the neighbor coordination number. . . . . . . . . . . . . . . . . . . . . 98

6.2 Values of the components of Ge activation strain tensors depending on the

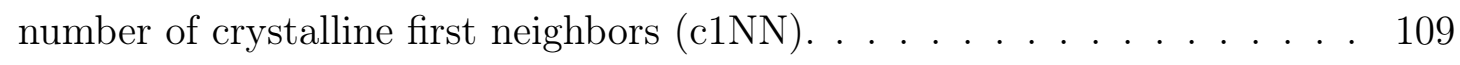


$\operatorname{ApPEndix} \Lambda$

\section{RUNNING MMONCA}

In this Appendix, a script used for simulating the full amorphization and SPER process in MMonCa is explained. With this script, the results shown in Figs. 6.6 and 6.8 can be reproduced. After that, the files with the parameterizations used in this thesis are also presented to show how the information described in previous Chapters is translated to MMonCa.

\section{A.1 Script Example}

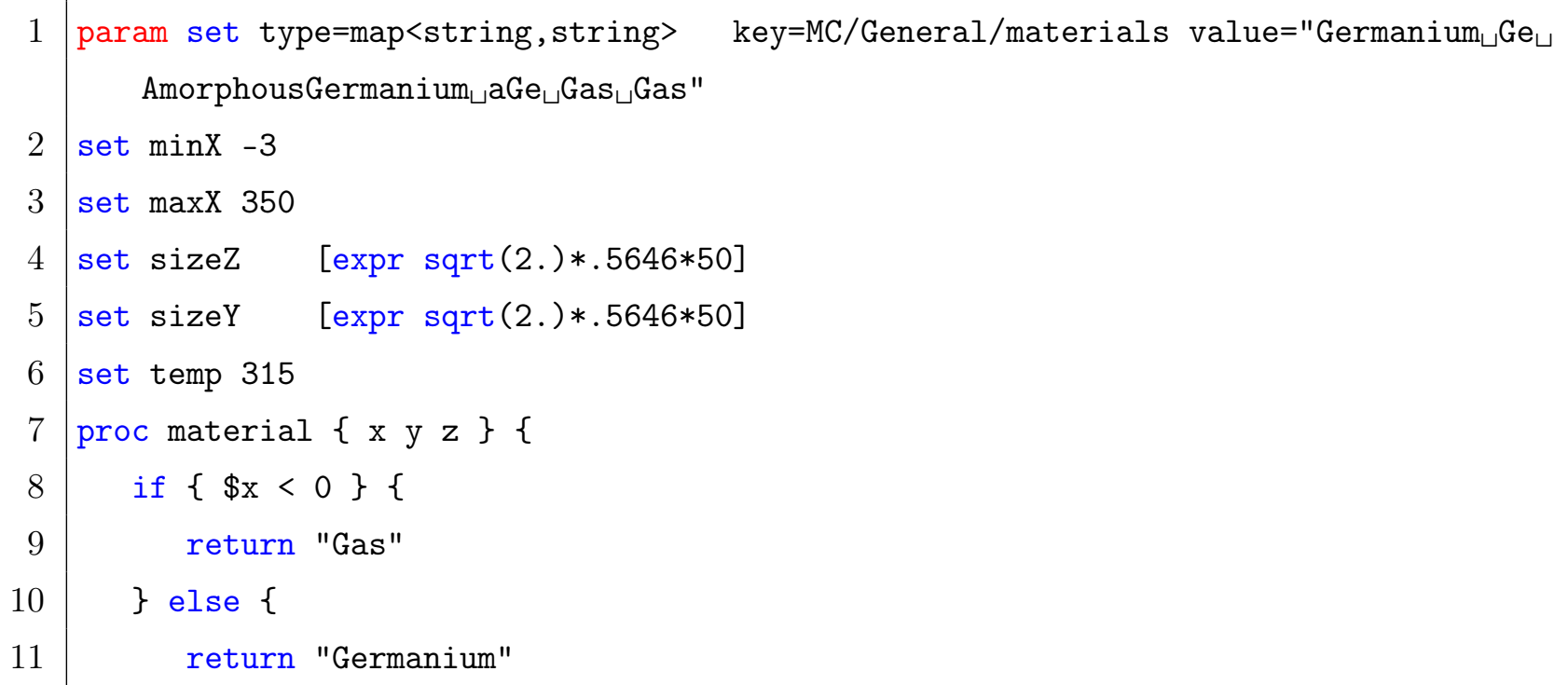




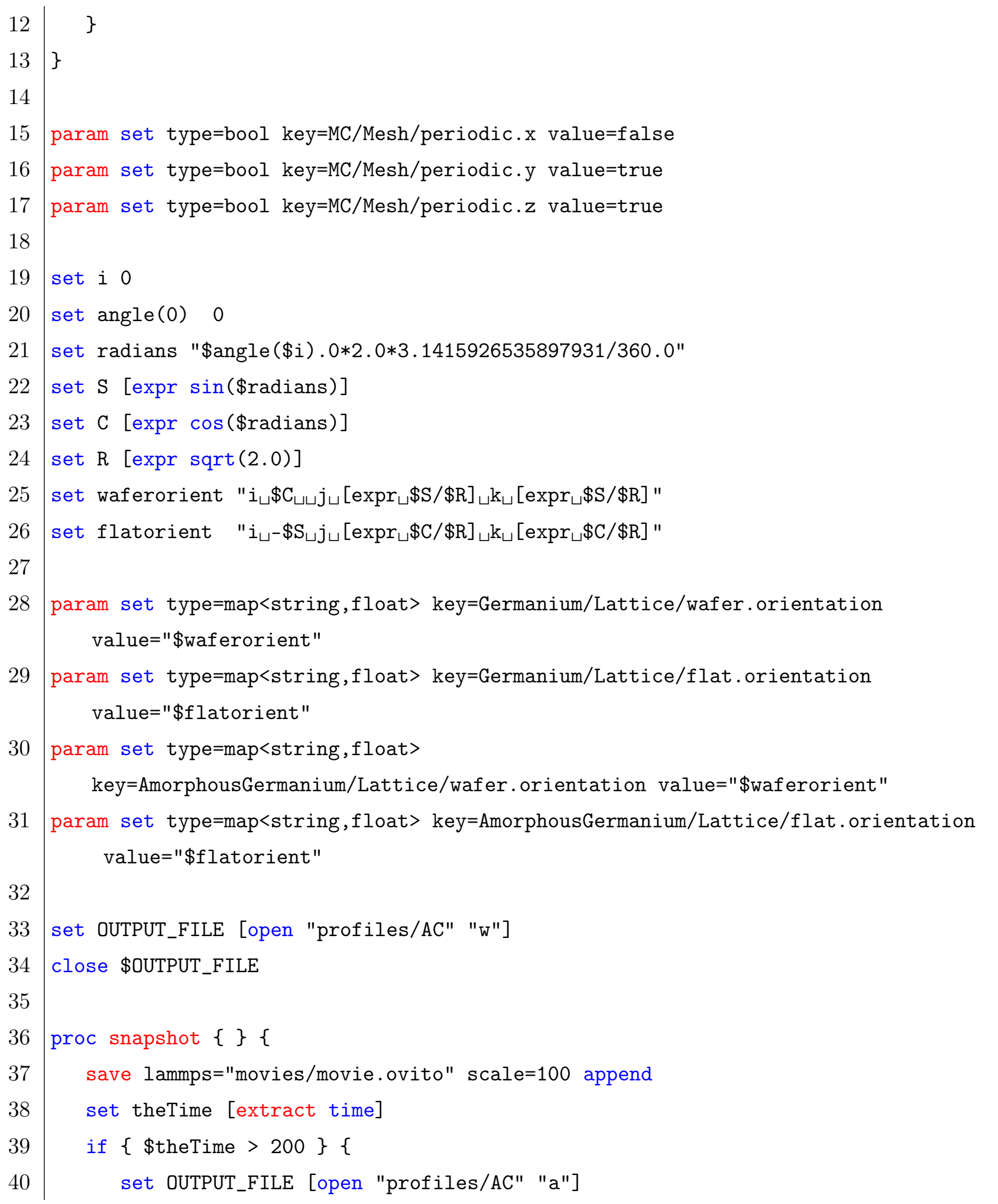




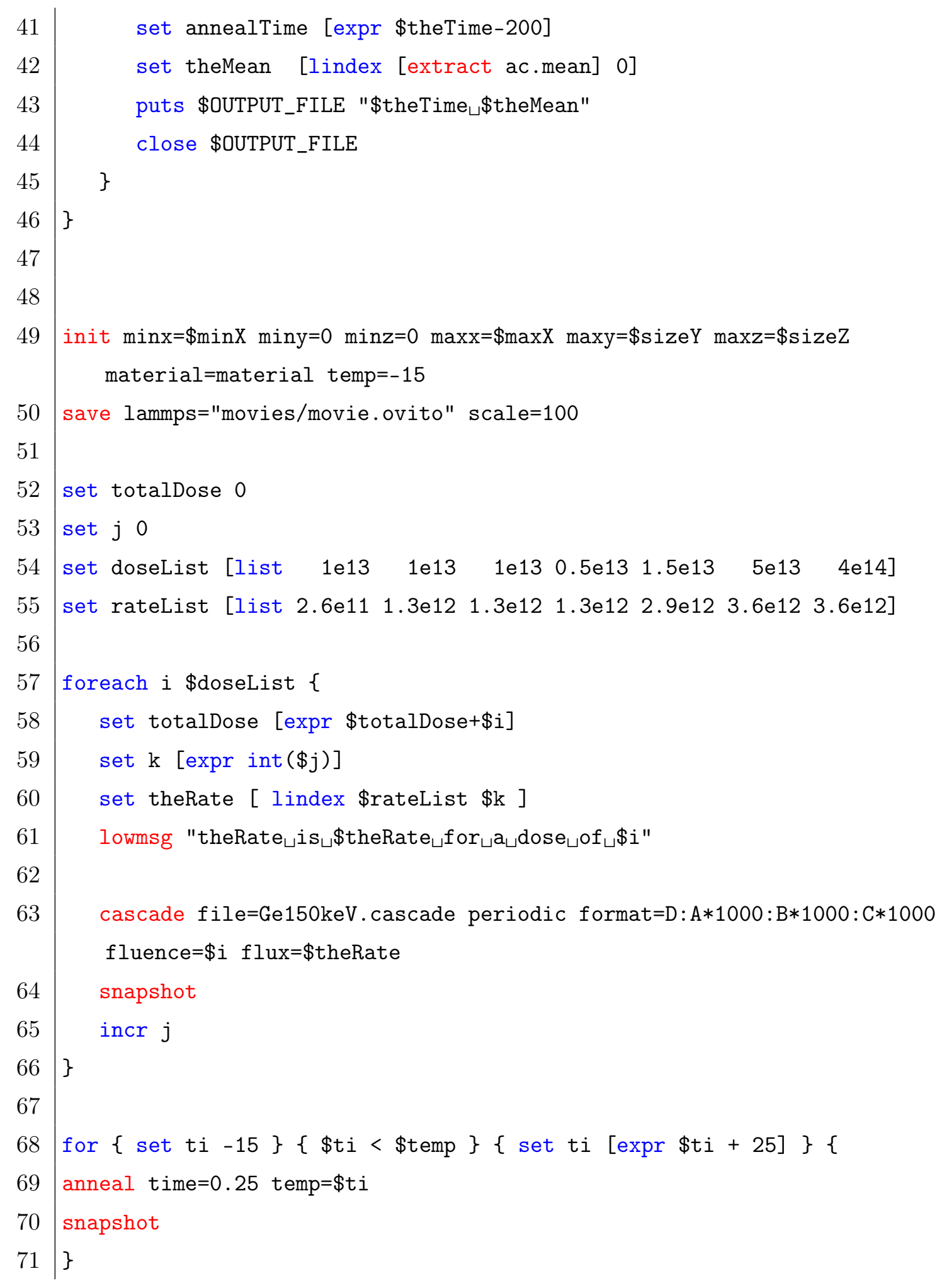


72

The main commands and lines are explained in the following. First, the materials that are going to be used in the simulations are defined. This saves time consumption, forcing MMonCa to only read the parameters needed for each simulation. In this case, we will only use Ge, $\alpha$-Ge, and Gas.

param set type=map<string, string >

AmorphousGermanium $a \mathrm{Ge}_{\sqcup} \mathrm{Gas}_{\sqcup} \mathrm{Gas} "$

Some Tcl variables are stored to its later use in temperature and space definition.

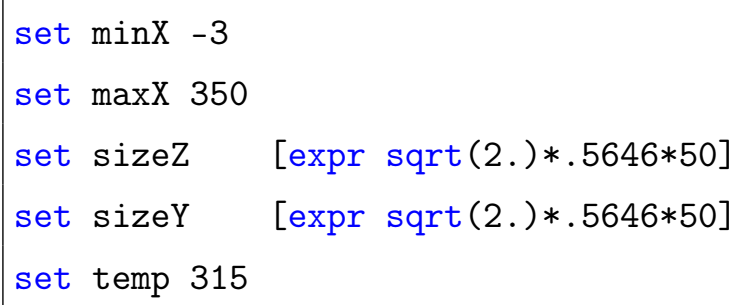

A Tcl procedure (function) will be called by MMonCa when defining the simulation box, to assign the materials to each Mesh Element. In this case, for every y and z, we want Gas to be assigned for $\mathrm{x}$ values lower than 0, and Germanium elsewhere. This will create a Gas-Germanium interface at $\mathrm{x}=0$. 


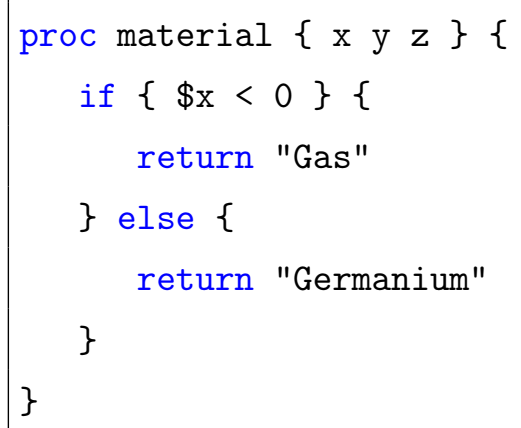

Then, periodic boundary conditions are defined for y and $\mathrm{z}$ axes only.

15

16

17

param set type=bool key=MC/Mesh/periodic. $\mathrm{x}$ value $=\mathrm{false}$

param set type=bool key=MC/Mesh/periodic. $\mathrm{y}$ value=true

param set type=bool key=MC/Mesh/periodic. $z$ value=true

In the next small definitions, the orientation of the wafer is communicated to MMonCa. Since a (001)Ge substrate is wanted, the following script lines might seem complicated for an angle of $0^{\circ}$. Nevertheless, it can be used for other angles by only changing the value of the variable angle.

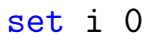
value=" $\$$ flatorient" 
key=AmorphousGermanium/Lattice/wafer.orientation value=" \$waferorient" param set type=map<string,float $>$ key=AmorphousGermanium/Lattice/flat.orientation value="\$flatorient"

The output file is initialized with the following lines.

A Tcl procedure can be defined to store the desired output information at the default times defined in MMonCa with the name snapshot. In this case we want to save the position of the simulated objects to be visualized by OVITO with a LAMMPS format. For times greater than the sum of the time spent implanting and in the annealing ramp, the $\alpha / \mathrm{c}$ interface position will be stored. This can be done only if we know in advance the nature of the interface. For example, if the information is extracted at early stages of the implantation in which two $\alpha / \mathrm{c}$ interfaces coexist, it will lead to wrong results. In such case, the extraction from its visualization in OVITO, for example, is recommended.

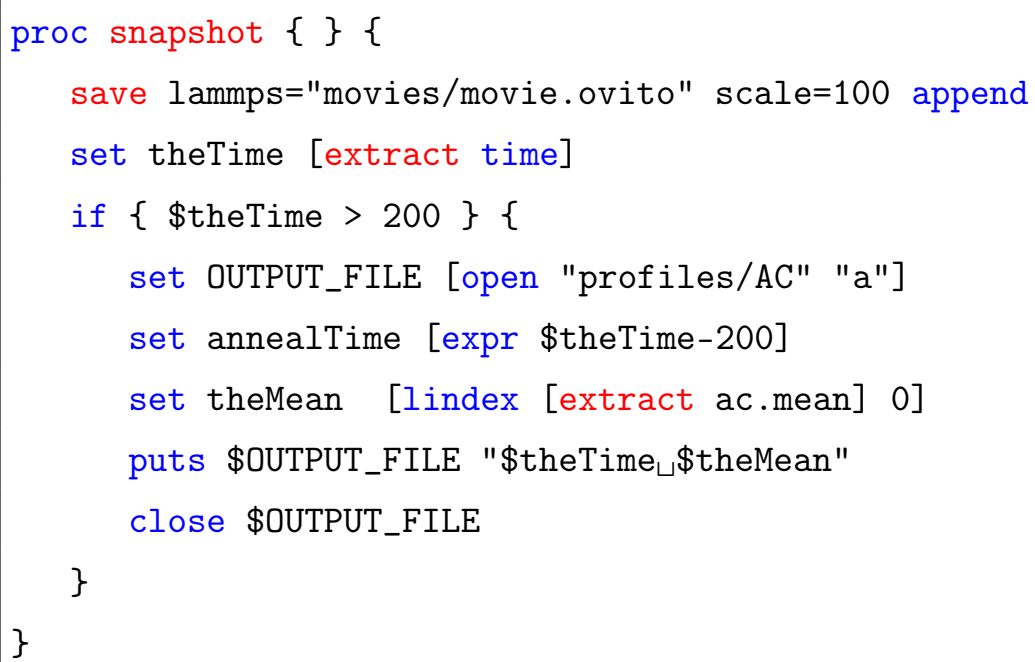

Now, we tell MMonCa to initialize the simulation box, its dimensions, the temperature and the spatial distribution previously defined. Later, we initialize the OVITO file, by 


\section{A.1 Script Example}

saving the first empty data file.

49

50

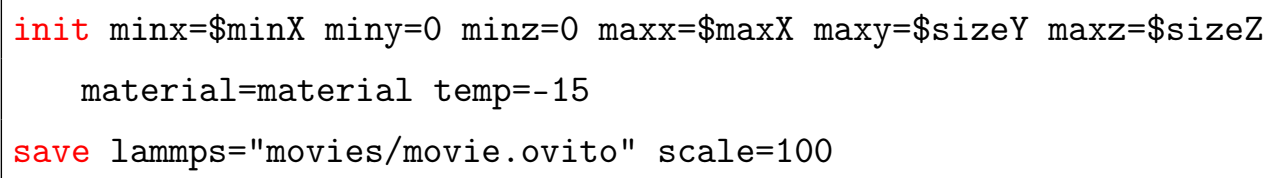

In the next loop, values for fluences (dose) are paired with different fluxes (dose.rate), since experimentally different fluxes were used. Then, the values are printed in the screen.

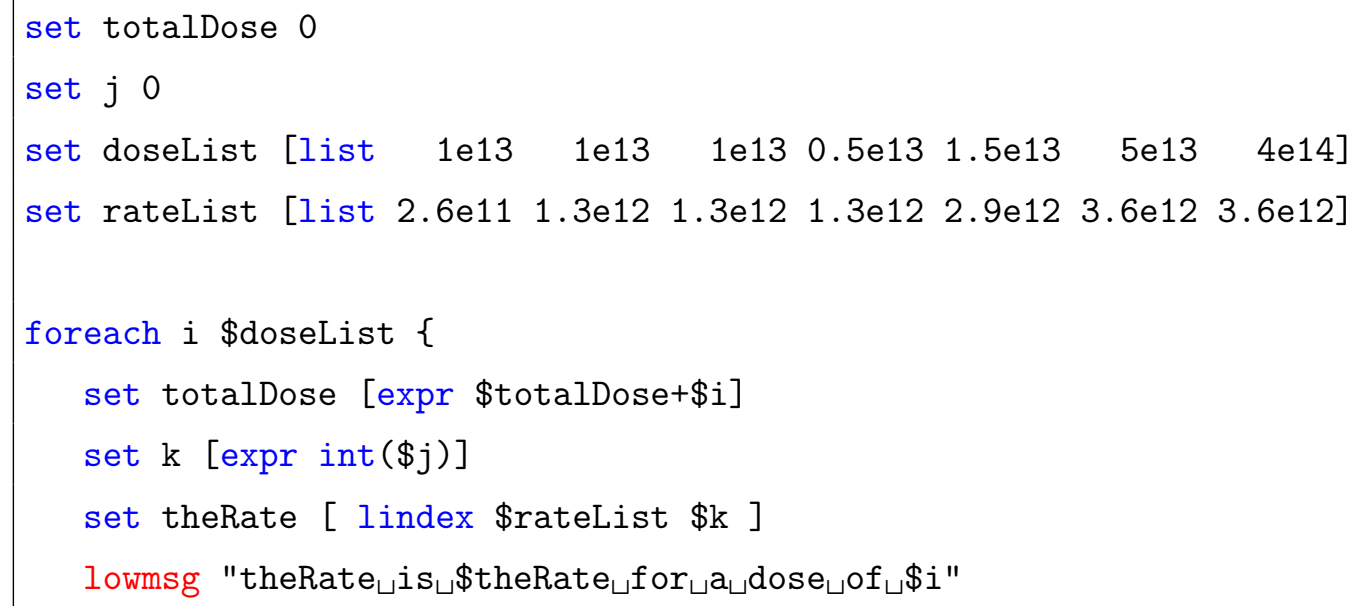

With those values, the implantation, from an external file are introduced in MMonca. Dynamic annealing time is calculated by dividing the fluence by the flux, and the damage evolution is computed. Then, snapshot procedure is manually called for each step (additionally to the automatic predefined calls).

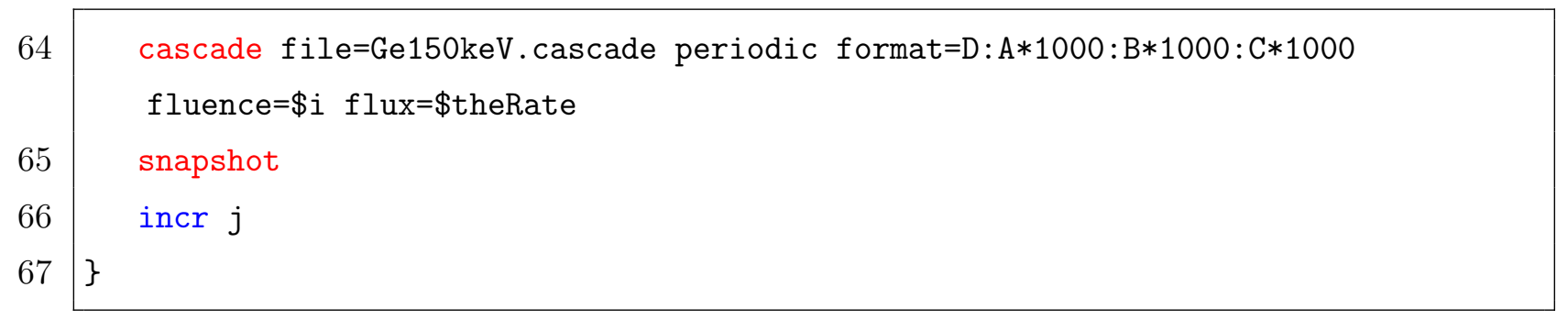

A typical annealing ramp is done to rise gradually the temperature, from $-15^{\circ} \mathrm{C}$ to the desired $315^{\circ} \mathrm{C}$. 
for $\{$ set ti -15$\}\{\$$ ti $<$ temp $\}\{$ set ti $[\operatorname{expr} \$ t i+25]\}\{$

Finally, the sample is annealed at the desired temperature for the reported experimental times.

76

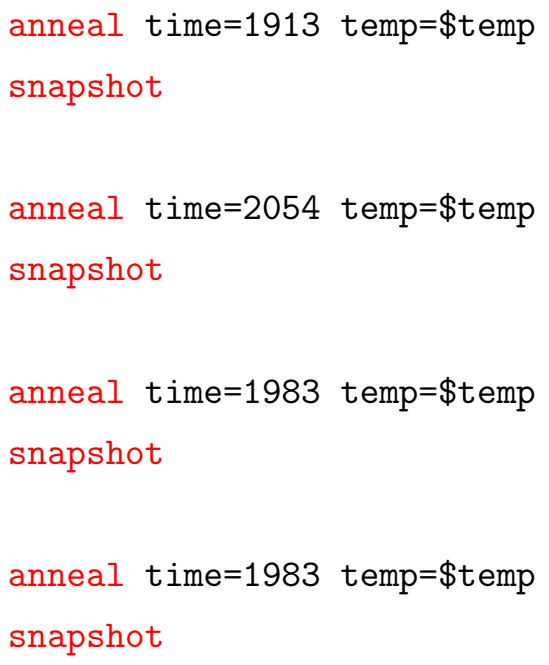

\section{A.2 Parameters}

All presented parameterizations presented in this Section are defined in config/Germanium/ folder. The respective files are indicated between brackets.

\section{Vacancies $\{$ Vacancy $\}$}

2 arrhenius $V($ formation) $\{1 \mathrm{e} 242.43\}$ 
Interstitials \{Germanium\}

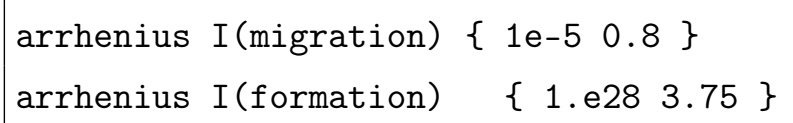

Amorphous Pockets \{IVCluster

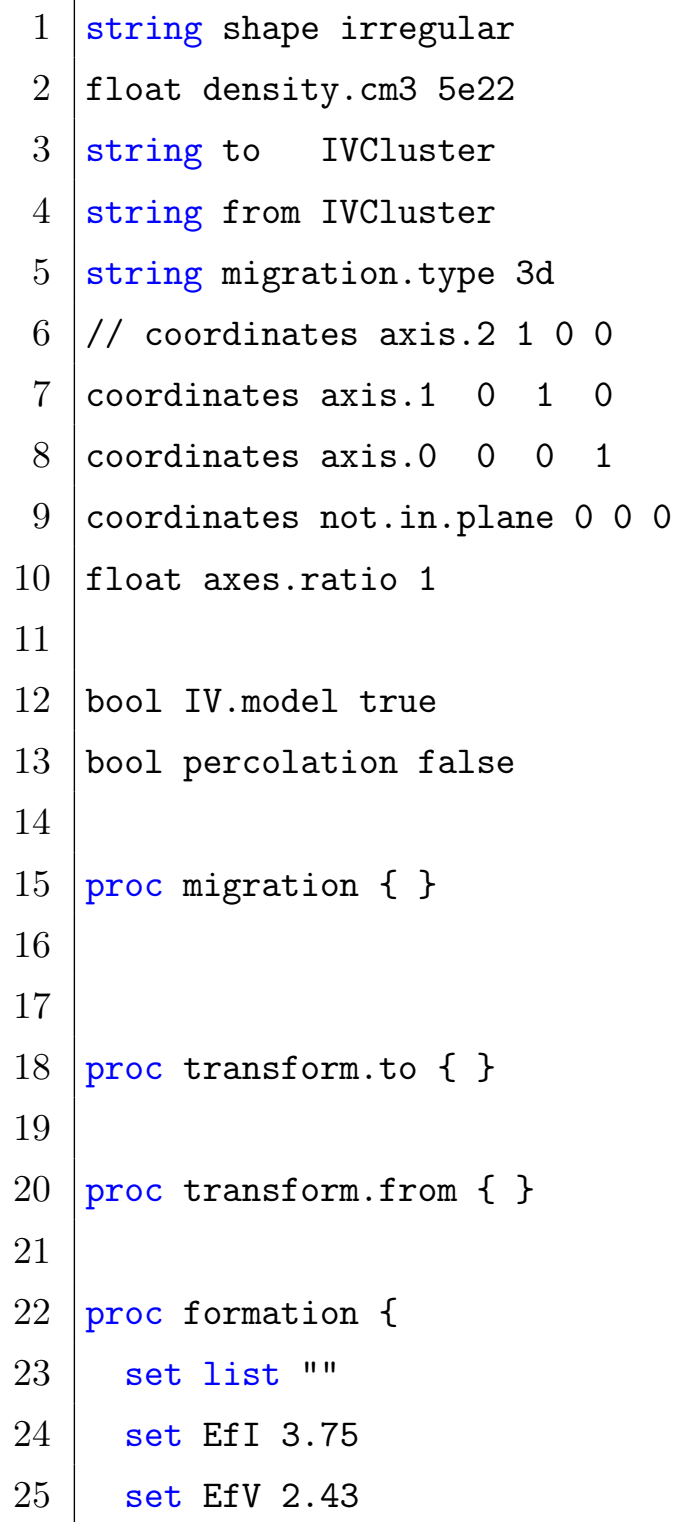




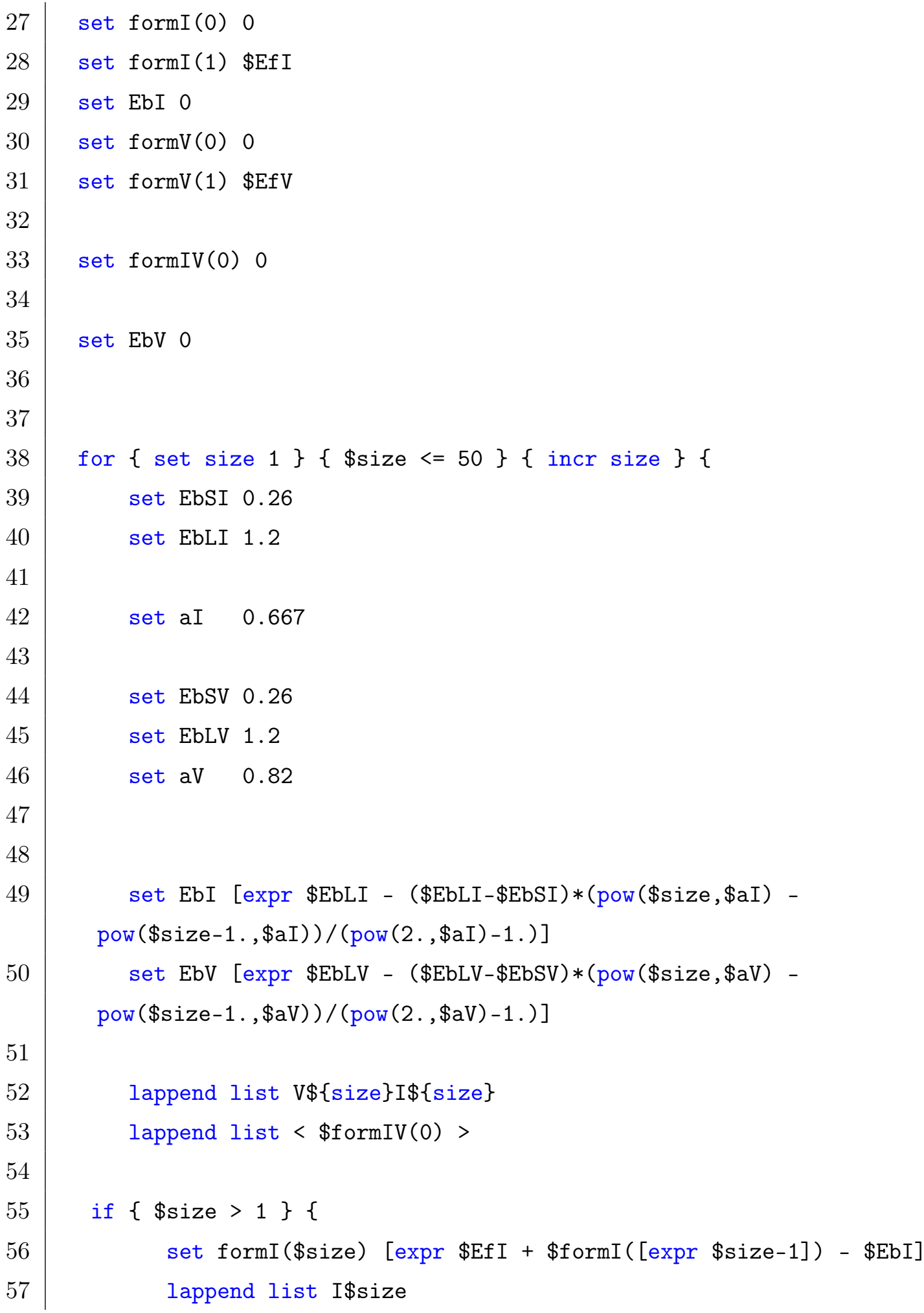




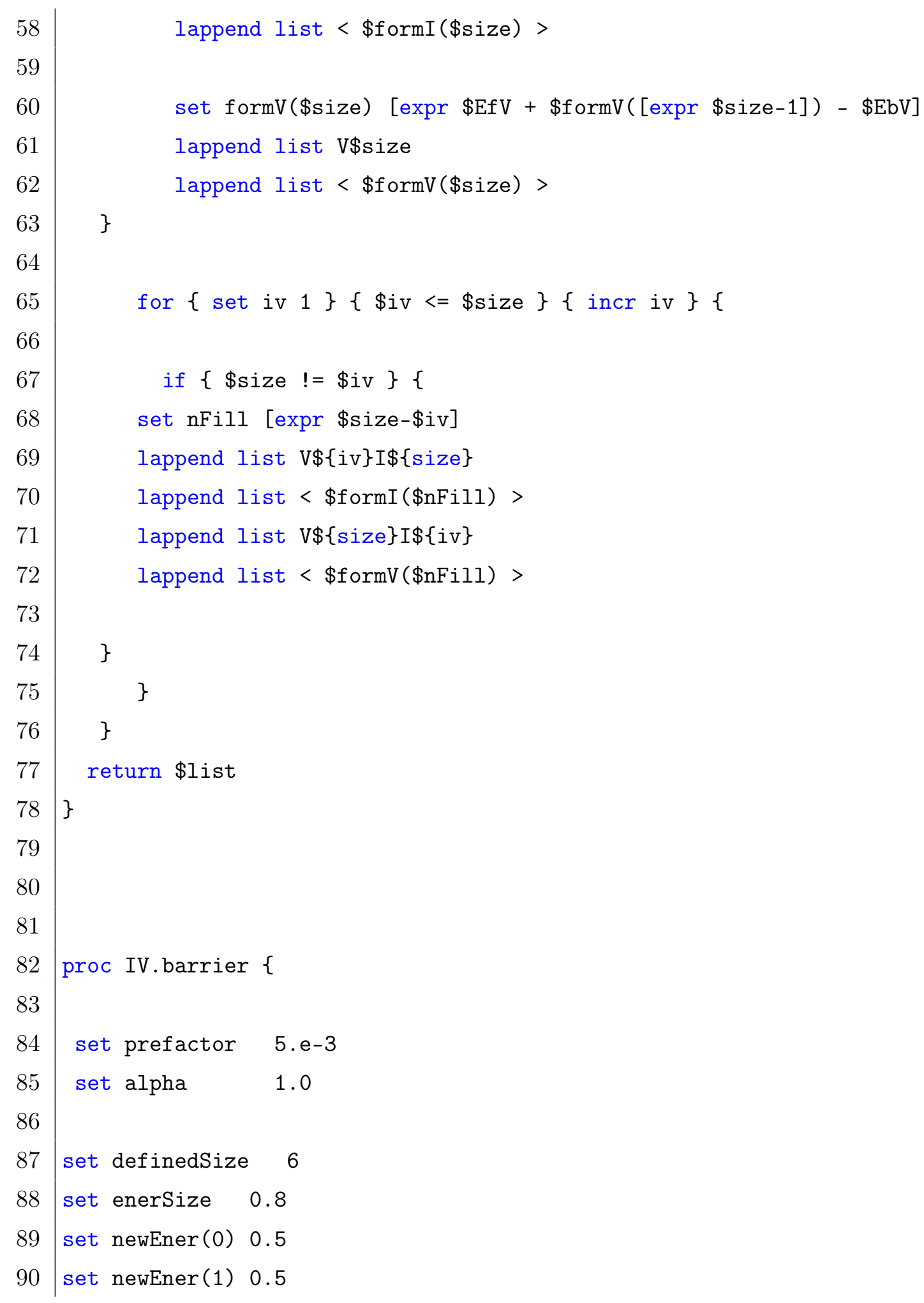




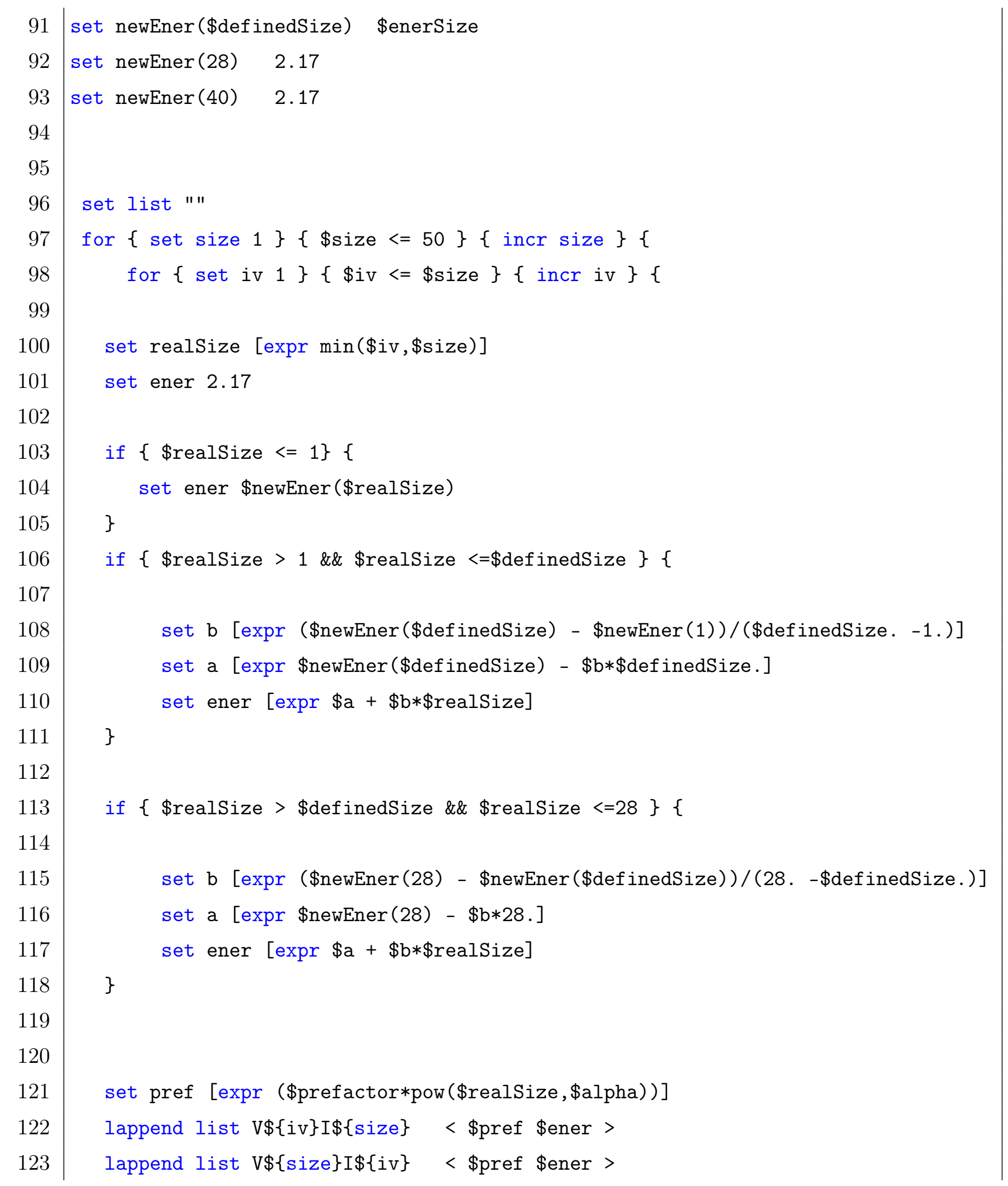




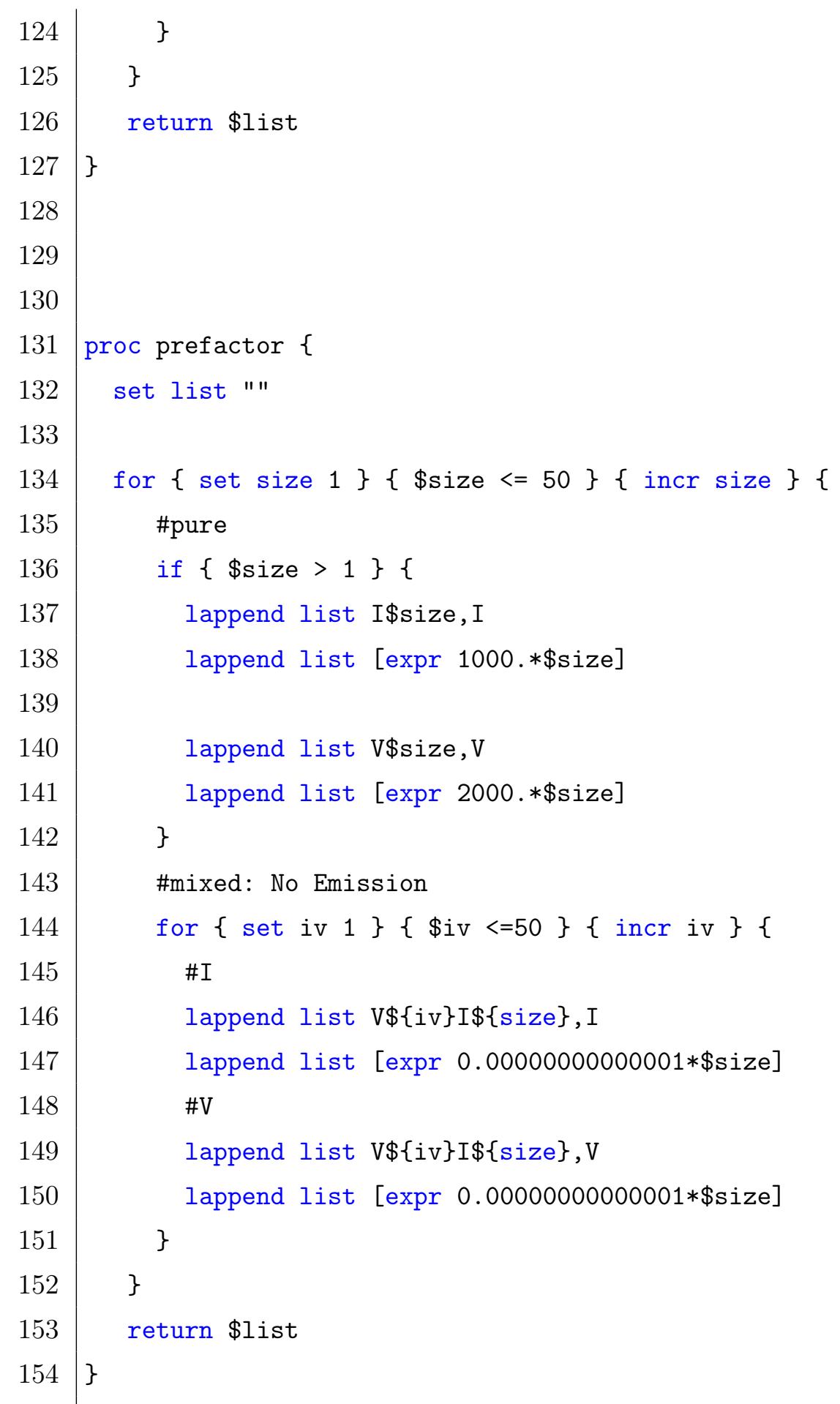




\section{SPER $\{$ SPER $\}$}

Here, the same needs to be defined for Amorphous Germanium material.

\begin{tabular}{|c|c|}
\hline float prefactor. 111 & $1.5 e 12$ \\
\hline float prefactor. 110 & $2.41 \mathrm{e} 16$ \\
\hline float prefactor. 100.6 & $1.176 \mathrm{e} 17$ \\
\hline float prefactor. 100.7 & $1.176 \mathrm{e} 17$ \\
\hline float prefactor. 100.8 & $1.176 \mathrm{e} 17$ \\
\hline float prefactor.100.9 & $2.35 \mathrm{e} 18$ \\
\hline float prefactor.100.10 & $2.35 \mathrm{e} 18$ \\
\hline & \\
\hline float energy & 2.17 \\
\hline float on.fly.lattice & 0.5646 \\
\hline \multicolumn{2}{|c|}{$/ / 1.00$ means a $50 \%$ probability } \\
\hline float twin.probability & 1.00 \\
\hline \multicolumn{2}{|l|}{ // Stress tensors } \\
\hline float dvpar.100.2 & -0.57 \\
\hline float dvperp.100.2 & 0 . \\
\hline float dvpar.100.3 & -45 \\
\hline float dvperp.100.3 & 23. \\
\hline
\end{tabular}


Appensix $B$

\section{PERSONAL CONTRIBUtions}

\section{Papers in journals}

- J.L. Gomez-Selles, A. Claverie, B. Sklenard, F. Benistant and I. Martin-Bragado, "Atomistic simulation of damage accumulation and amorphization in Ge", Journal of Applied Physics, 117, 055703 (2015).

- J.L. Gomez-Selles, B.L. Darby, K. S. Jones and I. Martin-Bragado,"Lattice kinetic Monte Carlo modeling of germanium solid phase epitaxial growth", physica status solidi (c), 11, 1, 93-96 (2014)

- I. Martin-Bragado, A. Rivera, G. Valles, J.L. Gomez-Selles and M.J. Caturla, "MMonCa: An Object Kinetic Monte Carlo simulator for damage irradiation evolution and defect diffusion", Computer Physics Communications, 184, 12, pp 2703-2710 (2013)

- B.L. Darby, B.R. Yates, I. Martin-Bragado, J.L. Gomez-Selles, R.G. Elliman and K.S. Jones, "Substrate orientation dependence on the solid phase epitaxial growth rate of Ge", Journal of Applied Physics, 113, 3, 033505 (2013) 


\section{Communications at Conferences}

- J.L. Gomez-Selles, A. Claverie, F. Benistant and I. Martin-Bragado, "Kinetic Monte Carlo modeling for full Ge processing", E-MRS 2015, Lille, France.

- I. Martin-Bragado, J.P. Balbuena, J.L. Gomez-Selles and A. Sarikov, "Atomistic modeling of epitaxial growth of semiconductor materials", E-MRS 2015, Lille, France.

- J.L. Gomez-Selles, A. Claverie, F. Benistant and I. Martin-Bragado, "Modeling of damage accumulation and dissolution mechanisms in ion-implanted Ge", IEEENMDC 2014. Aci Castello, Italy.

- I. Martin-Bragado, J.L. Gomez-Selles and B. Sklenard, "Lattice Kinetic Monte Carlo modeling of Solid Phase Epitaxial Regrowth for silicon and germanium materials", E-MRS 2014, Lille, France.

- J.L. Gomez-Selles, A. Claverie, B. Sklenard, F. Benistant and I. Martin-Bragado, "Comprehensive modeling of amorphization in Ge using Kinteic Monte Carlo", EMRS 2014, (Poster). Lille, France.

- J.L. Gomez-Selles, B. Sklenard, F. Benistant and I. Martin-Bragado, "Ion-Beam induced damage in Ge: an atomistic approach", COSIRES 2014, Alicante Spain.

- J.L. Gomez-Selles, B.L. Darby, K. S. Jones and I. Martin-Bragado, "Lattice kinetic Monte Carlo modeling of germanium solid phase epitaxial growth". E-MRS 2013, Strasbourg, France.

\section{Stays abroad}

- Centre d'Élaboration de Matériaux et d'Études Structurales, Centre National de la Recherche Scientifique (CEMES-CNRS), Toulouse, France. From 10/01/2015 to $10 / 04 / 2015$.

\section{Modular Monte Carlo simulator: MMonCa}

The presented investigations have been possible by actively participating in the development, implementation and calibration of new and existing models into an Open Source simulator opened to the scientific community for both industrial and research use. MMonCa 
is an Object Oriented simulator, programmed in $\mathrm{C}++$ language with a $\mathrm{Tcl} / \mathrm{Tk}$ user interface. Thus, the appliance and implementation of new models and calibration of those or new ones into MMonCa requires hands-on skills in the mentioned languages, which have been acquired during this work. Unix-based scripting tools (awk,sh) have also been used. Version control of the code has been carried out through Git utilities. 
